\title{
Self-assembled \\ Vertically Aligned Nanocomposites \\ for \\ Solid-State Batteries
}

Daniel M. Cunha 


\section{Self-assembled Vertically Aligned Nanocomposites for Solid-State Batteries}

Daniel Monteiro Cunha 


\section{Cover}

The cover shows a cross-sectional Scanning Electron Microscopy of the $\mathrm{LiMn}_{2} \mathrm{O}_{4}$ and $(\mathrm{Li}, \mathrm{La}) \mathrm{TiO}_{3}$ vertically aligned nanocomposite grown on crystalline $\mathrm{Nb}: \mathrm{SrTiO}_{3}$ with the (110) orientation.

\section{Graduation Committee}

\section{Chairman and Secretary}

Prof. Dr. J.L. Herek (University of Twente)

\section{Supervisors}

Prof. Dr. Ir. M. Huijben (University of Twente)

Prof. Dr. Ing. A.J.H.M. Rijnders (University of Twente)

\section{Members}

Prof. Dr. Ir. J.E. ten Elshof (University of Twente)

Prof. Dr. Ir. J.W.M. Hilgenkamp (University of Twente)

Prof. Dr. T. Hitosugi (Tokyo Institute of Technology)

Prof. Dr. J. MacManus-Driscoll (University of Cambridge)

Prof. Dr. F.G. Mugele (University of Twente)

The research presented in this thesis was carried out at the Inorganic Materials Science Group, Nanoelectronic Materials and Thin Films Cluster, Department of Science and Technology, MESA+ Institute of Nanotechnology at the University of Twente, The Netherlands. The research was financially supported by The Netherlands Organization for Scientific Research (NWO).

Self-assembled Vertically Aligned Nanocomposites for Solid-State Batteries

Ph.D thesis, University of Twente, Enschede, The Netherlands

Copyright (C) 2021 by D. Monteiro Cunha

DOI: $10.3990 / 1.9789464212242$

ISBN: 978-94-6421-224-2

Printed by IPSKamp Printing, Enschede, The Netherlands 


\title{
SELF-ASSEMBLED VERTICALLY ALIGNED NANOCOMPOSITES FOR SOLID-STATE BATTERIES
}

\author{
DISSERTATION
}

to obtain

the degree of doctor at the Universiteit Twente, on the authority of the rector magnificus, prof. dr. ir. A. Veldkamp, on account of the decision of the Doctorate Board to be publicly defended on Wednesday 10 March 2021 at 12.45 hours

by

\section{Daniel Monteiro Cunha}

born on the 16th of July, 1990 in Sao Paulo, Brazil 
This dissertation has been approved by:

Prof. Dr. Ing. A.J.H.M. Rijnders (University of Twente)

Prof. Dr. Ir. M. Huijben (University of Twente) 


\section{Contents}

1 Introduction 1

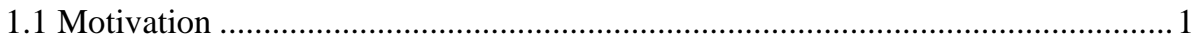

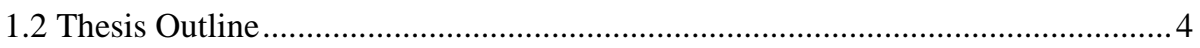

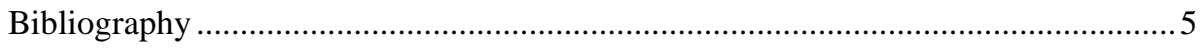

2 The building blocks of an All-Solid-State battery 9

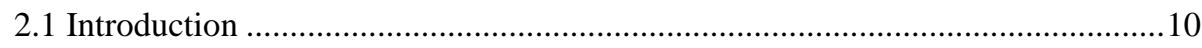

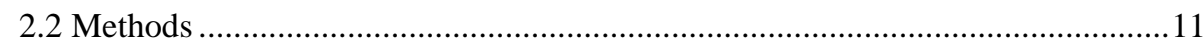

2.3 Orientation-dependent intercalation kinetics for $\mathrm{Li}_{4} \mathrm{Ti}_{5} \mathrm{O}_{12}$ anode thin films.......12

2.3.1 Control over the LTO crystal orientation ............................................13

2.3.2 Electrochemical behavior of epitaxial LTO films .................................16

2.3.3 Film thickness dependence on electrochemistry..................................17

2.3.4 Phase-field modelling of the lithiation mechanism ................................20

2.4 Tailoring ionic transport by control of crystal orientation in spinel $\mathrm{LiMn}_{2} \mathrm{O}_{4}$

thin film cathode ................................................................................. 22

2.4.1 LMO thin film growth and characterization ......................................24

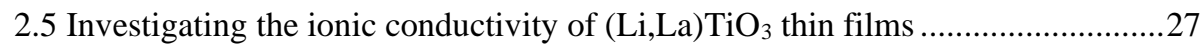

2.5.1 Temperature dependence analysis for LLTO ionic conductivity................30

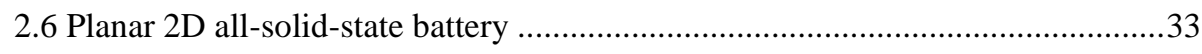

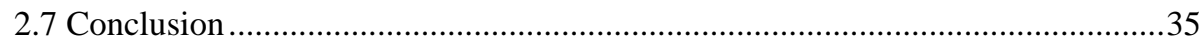




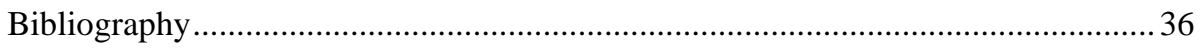

3 Morphology Evolution during Li-based VAN Growth 43

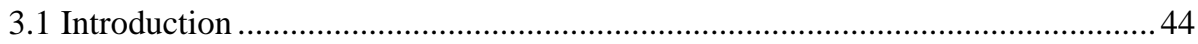

3.2 PLD growth and structural analysis of VAN thin films .................................. 45

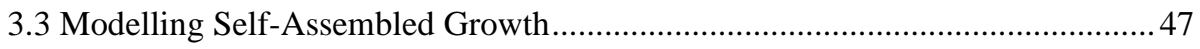

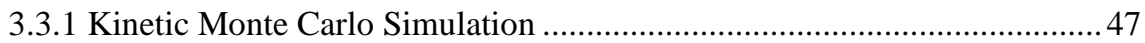

3.3.2 KMCS model with experimental activation energies and higher degrees

of freedom for hopping ................................................................. 49

3.4 Comparison between experimental and simulation results.............................53

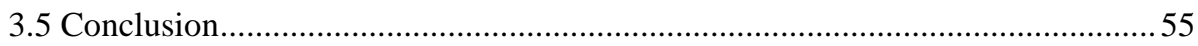

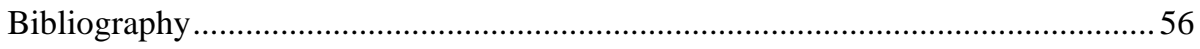

4 Li-based Vertically Aligned Nanocomposites $\quad 61$

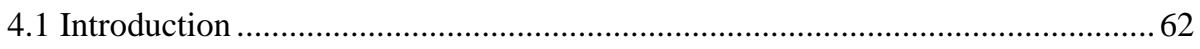

4.1.1 Conditions and Mechanisms of VAN Film Growth ................................ 63

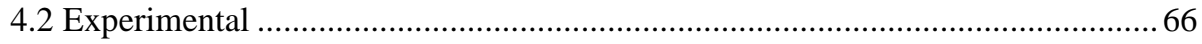

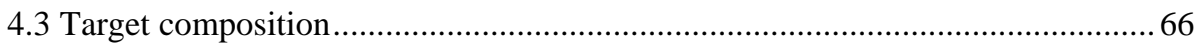

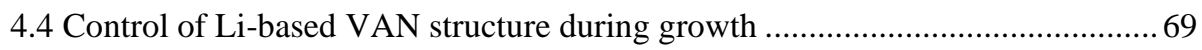

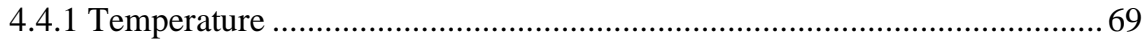

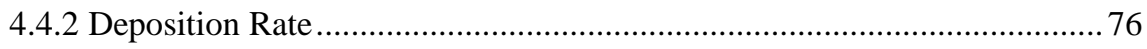

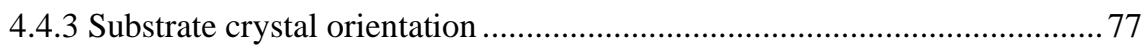

4.5 Investigating the influence of different conductive buffer layers ........................ 84

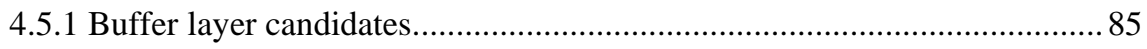

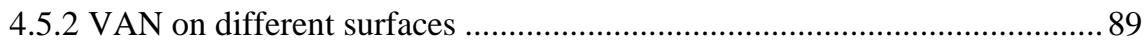

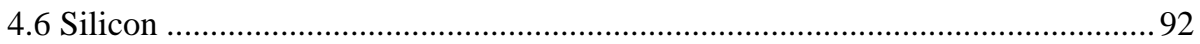




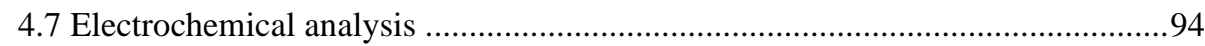

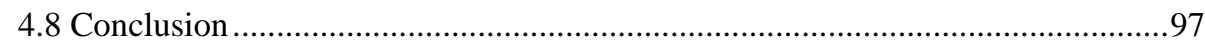

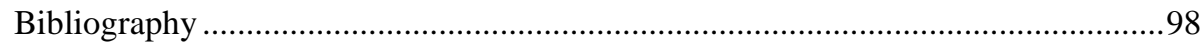

$\begin{array}{lr}5 \text { Electrochemistry at the Nanoscale } & 105\end{array}$

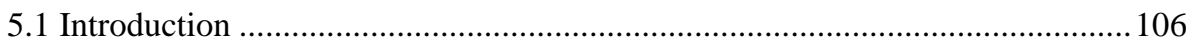

5.2 First Order Reversal Curve Current-Voltage ............................................. 107

5.2.1 Parasitic Capacitance ........................................................................ 108

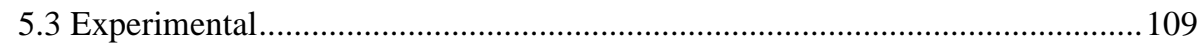

5.4 Local Electrochemistry in $\mathrm{LiMn}_{2} \mathrm{O}_{4}$ cathode thin films.............................. 110

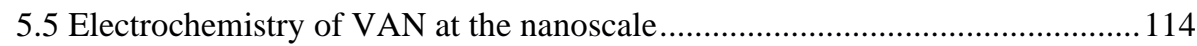

5.7 Prospects for the analysis of a complex ionic/electronic system ....................116

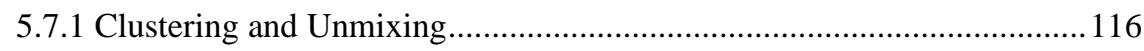

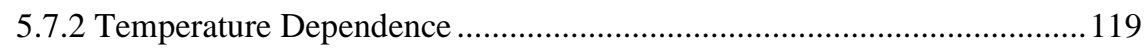

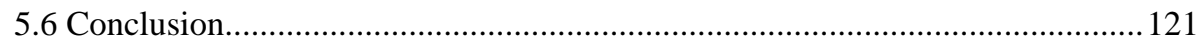

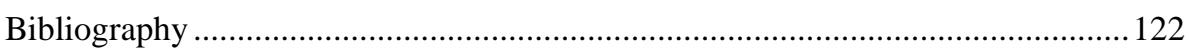

$\begin{array}{ll}\text { Summary } & 125\end{array}$

$\begin{array}{lr}\text { Samenvatting } & 129\end{array}$

$\begin{array}{ll}\text { List of Publications } & 133\end{array}$

$\begin{array}{ll}\text { Acknowledgements } & 135\end{array}$ 



\section{Chapter 1}

\section{Introduction}

\subsection{Motivation}

Since its introduction in 1991 by Sony, Lithium-ion (Li-ion) batteries are the most popular rechargeable batteries [1] as they have become the main power source for many applications, such as portable electronics, power tools, and hybrid/full electric vehicles. Tremendous research effort has been devoted to investigate the electrochemical performance of a wide variety of active lithium-based materials to develop batteries with large capacity, high energy and power density, improved safety, long cycle-life, fast response and low cost. Despite the efforts, none of the current rechargeable batteries can fully satisfy all the challenging requirements for the projected energy storage needs. Key problems for this limitation include slow electrode process kinetics with high polarization and a low rate of ionic diffusion or electronic conductivity, particularly at the electrodeelectrolyte interfaces.[2-4]

Commercial lithium ion batteries have an energy density of $300-500 \mathrm{mWh} \cdot \mathrm{cm}^{-3}$, which is still far below the theoretical energy density of lithium-air batteries $\left(2800 \mathrm{mWh} \cdot \mathrm{cm}^{-3}\right)$.[5] Common rechargeable batteries are based on liquid electrolytes, which results in several restrictions for their design and size due to the available separators. Secondly, these acidic liquids cause unwanted reactions at the electrode surfaces, reducing the stability of the battery. Finally, these liquids carry the inherent risk of leakage and explosion. Therefore, the need for all solid-state microbatteries arises, which will show enhanced safety, volumetric energy/power density and chemical stability. These microbatteries, developed by thin-film architecture, enables the powering of micro-scale devices, such as stand-alone sensor systems for internet of things, implantable medical devices, labs-on-chip, and credit cards.[6]

One of the main issues with state-of-the-art solid-state electrolytes is the poor ionic conductivity compared to liquid organic electrolytes. Increasing the ionic conductivity of solid electrolytes is therefore an essential step to make further progress in this direction. 
However, while some promising solid electrolytes with lithium conductivities approaching those of liquid electrolytes have recently been reported for sulfide conductors (e.g. $\mathrm{Li}_{7} \mathrm{P}_{3} \mathrm{~S}_{11}, \mathrm{Li}_{10} \mathrm{GeP}_{2} \mathrm{~S}_{12}$ and $\mathrm{Li}_{9.54} \mathrm{Si}_{1.74} \mathrm{P}_{1.44} \mathrm{~S}_{11.7} \mathrm{Cl}_{0.3}$ ),[7] stability issues limit the ionic transport across the electrode-electrolyte interface. In contrast, promising oxide electrolytes (e.g. perovskite $\mathrm{La}_{0.5} \mathrm{Li}_{0.5} \mathrm{TiO}_{3}$, garnet $\mathrm{Li}_{7} \mathrm{La}_{3} \mathrm{Zr}_{2} \mathrm{O}_{12}$, LiPON $\left(\mathrm{Li}_{2.88} \mathrm{PO}_{3.73} \mathrm{~N}_{0.14}\right)$ and LISICON $\left(\mathrm{Li}_{14} \mathrm{ZnGe}_{4} \mathrm{O}_{16}\right)$ ), [7-9] with high chemical stabilities are currently limited by high grain boundary resistances. Hence, a dramatic reduction of the thickness of the solid electrolyte, provided by thin-film technology, is required to overcome the limited lithium conductivity to enable fast charge-discharge rates.

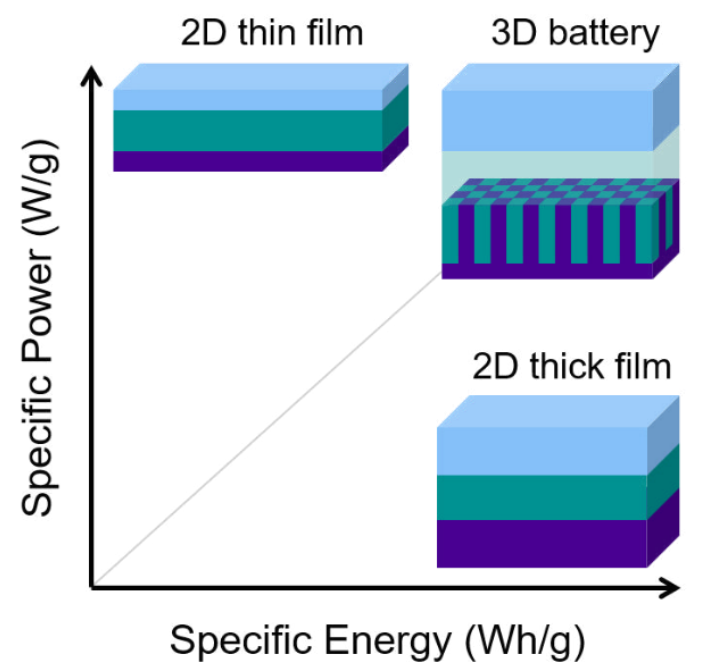

Figure 1-1. Ragone plot of the solid-state battery designs, illustrating the improvement of a 3D system over the 2D planar films.

Planar 2D solid-state thin-film batteries exhibit an undesirable energy vs. power balance, which can be improved by the application of 3D geometries. An additional advantage of these 3D batteries is that the internal surface area between cathode, electrolyte and anode is enlarged, improving their current output. This will ensure a giant step in power and energy density for solid-state devices, as depicted in Figure 1-1, allowing for a much better energy storage performance.[10-12] Several concepts for a $3 \mathrm{D}$ microbattery layout have been proposed in previous studies, based on membrane templates, interdigitated microrods, porous aerogels, microchannel plates and anisotropic etching.[11,13] However, most of these designs are only conceptual and have only been focusing on partial solid-state devices. Furthermore, fabrication of such 3D batteries relies presently on the use of costly methods, such as micro- and photolithography, or electrodeposition techniques combined with spin coating/infiltration. Therefore, the benefits of 3D batteries can only be fully exploited in the future if a synthetic route provides structure control of such systems down to the tens of nanometers length scales 
in combination with tunable crystal orientations of the individual materials and their shared interfaces.

The advantages of nanostructured materials are larger electrode/electrolyte contact area leading to higher (dis)charge rates, short path lengths for both electronic and Li-ion transport leading to higher charge flow, and better accommodation of the strain during lithium insertion/extraction. Various studies on Li-ion batteries have demonstrated that nanocrystalline intermetallic alloys, nanosized composite materials, carbon nanotubes, and nanosized transition-metal oxides are all promising new anode materials, while nanosized high-voltage cathodes $\mathrm{LiCoO}_{2}, \mathrm{LiFePO}_{4}$ and $\mathrm{LiMn}_{2} \mathrm{O}_{4}$ show higher capacity and better cycle life than their usual larger-particle equivalents.[14]

Nanocomposites have attracted great interest over the last decades due to the presence of enhanced functional material properties induced by confinement of the structural dimensions.[15] Ceramics-based nanocomposites is a growing research area,[16] as they are currently being used in a wide range of applications, such as motor engines, heat exchangers, and aircraft/spacecraft technology. However, accurate control of the distribution and orientation of the nanoparticles within the matrix material is often limited or impossible. Detailed knowledge on the alignment of nanostructures through selfassembly is very well studied in organic systems,[17] but remains a rather unexplored territory for inorganic, ceramic nanocomposites.

In parallel to planar heterointerfaces, vertical heteroepitaxial (Figure 1-1) nanocomposite thin films have been developed in the past decade as a new materials' platform for creating self-assembled device architectures and multifunctionalities, as they show a wide range of attributes arising from the strong interplay among structural, electronic, magnetic, and even ionic properties.[18-21] Such epitaxial vertically-aligned nanocomposites (VANs) offer promising advantages over conventional planar multilayers as key functionalities are tailored by the strong coupling between the two phases and their interfaces, such as strain-enhanced ferroelectricity and multiferroics,[22,23] enhanced ferromagnetism,[24] magnetoresistance,[25] electronic transport,[26] and coupled dielectric and optical effects.[27]

Self-assembled vertically aligned nanocomposite thin films with two immiscible oxides can exhibit unique properties that are not present in the single-phase materials, because of the strong interfacial coupling at the vertical phase boundaries. The immiscibility of the phases, e.g. perovskite-spinel combinations, forms the foundation of the self-assembly procedure resulting in nanopillar/matrix structures. Epitaxial VANs are self-assembled through physical vapor deposition (PVD), without control of the deposition sequence, as is required for planar multilayer films. For epitaxially directed self-assembly, it is desirable that one phase in the film is crystallographically wellmatched to the substrate such that it nucleates, grows epitaxially and forms the host matrix, while the second phase (epiphyte) epitaxially aligns with the matrix phase. The host and epiphyte can both be chosen to be active phases whose functional properties are 
of interest, and where the phases interact with each other via strain- or charge-coupling at the interfaces. Dimensional tunability has been demonstrated, in which various nanopillar shapes and dimensions $(2-200 \mathrm{~nm})$ have been achieved in specific material systems.[20,21]

Although various epitaxial VANs have been studied in the last decade,[20,21] no lithium-based VANs have yet been explored for energy storage. The successful realization of two-phase epitaxial VANs has remained limited to specific material combinations: e.g. ferroelectrics $\left(\mathrm{BaTiO}_{3}, \mathrm{BiFeO}_{3}, \mathrm{PbTiO}_{3}\right)$ with ferromagnets $\left(\mathrm{CoFe}_{2} \mathrm{O} 4\right.$, $\left.\mathrm{NiFe}_{2} \mathrm{O} 4, \quad \mathrm{MgFe}_{2} \mathrm{O} 4, \mathrm{La}_{0.7} \mathrm{Sr}_{0.3} \mathrm{MnO}_{3}, \quad \mathrm{Fe}_{3} \mathrm{O}_{4}\right) ; \quad \mathrm{BiFeO}_{3}$ ferroelectric with $\mathrm{LaFeO}_{3}$ antiferromagnet; and $\mathrm{ZnO}$ insulator with $\mathrm{La}_{0.7} \mathrm{Sr}_{0.3} \mathrm{MnO}_{3}$ ferromagnet.[28,29] Only a limited number of studies have investigated the ionic conductivity of oxygen ions in such oxide-based epitaxial VANs for solid oxide fuel cells.[30]

Considering that self-assembled VANs are obtained through PVD techniques for perovskite-spinel systems, the main goal of this thesis is to apply the same principle for lithium containing oxide materials, and to study the impact on the electrochemical behavior for battery applications. The research is divided in small projects with different goals, as presented in the following section, not only to provide a comprehensive analysis on how different growth conditions alter the materials' structure and performance, but also to introduce methods to model the growth of such systems and measure the electrochemical properties at the nanoscale.

\subsection{Thesis Outline}

Detailed understanding of the electrochemical behavior of specific crystal facets of battery materials can only be obtained when a single type of crystal orientation interfacing the electrolyte can be synthesized. To elucidate this, in chapter 2 the control over the crystallographic properties of thin films via Pulsed Laser Deposition (PLD) was investigated to study the relation between crystal orientation and ionic and electronic conduction. This chapter is divided in four parts where the orientation-dependent intercalation kinetics for epitaxial $\mathrm{Li}_{4} \mathrm{Ti}_{5} \mathrm{O}_{12}$ (LTO) thin film anode is analyzed, where the influence of surface area on the capacity of the films is demonstrated. Subsequently, the crystal structure of the cathode material $\mathrm{LiMn}_{2} \mathrm{O}_{4}(\mathrm{LMO})$ was tailored by the orientation of the underlying single-crystalline substrate, and the resultant electrochemical performance was investigated. Furthermore, the influence of different PLD growth conditions on the $\mathrm{Li}_{0.5} \mathrm{La}_{0.5} \mathrm{TiO}_{3}$ (LLTO) solid-state electrolyte structure and ionic diffusion was studied. At last, a full planar solid-state battery was created, and its structural and electrochemical properties were investigated.

Since no lithium-based VANs have yet been explored for energy storage, in chapter 3 a model was developed to predict the formation of these structures, and to analyze what is the influence of temperature and deposition rate on the morphology evolution of these 
nanocomposites. Consisting of the promising LMO cathode and LLTO electrolyte, a Kinetic Monte-Carlo Simulation (KMCS) [31,32] was applied to confirm the possibility of modelling the self-assembled growth of VANs using activation energies obtained experimentally and with minimum restrictions for hopping directions.

In chapter 4 , to verify the control over the crystallographic properties of VAN thin films and how different growth conditions, e.g. substrate temperature, substrate orientation, and laser frequency, would influence on the crystal structure, morphology and composition of the nanopillars and matrix, the self-assembly procedure was applied for the first time with lithium containing materials to create electrode/electrolyte nanocomposites, deposited on crystalline substrates by PLD.

Lastly, advanced Scanning Probe Microscopy (SPM) techniques allow the measurement of electrochemistry on the nanoscale, which can be used to elucidate structure/function relationships in battery materials with exceptional resolution. To achieve insight into the non-uniform distribution of lithium activity, First Order Reversal Curve current-voltage (FORC-IV) [33,34] analysis was applied. To explain the conduction mechanisms that rule the electrochemistry in the nanoscale, the local ionic diffusion in LMO and VANs epitaxial thin films was studied in chapter 5.

\section{Bibliography}

[1] Li, M., Lu, J., Chen, Z., Amine, K., 30 Years of Lithium-Ion Batteries. Adv. Mater. 2018, $30,1-24$.

[2] Luntz, A. C., Voss, J., Reuter, K., Interfacial Challenges in Solid-State Li Ion Batteries. J. Phys. Chem. Lett. 2015, 6, 4599-4604.

[3] Wang, K. X., Li, X. H., Chen, J. S., Surface and interface engineering of electrode materials for lithium-ion batteries. Adv. Mater. 2015, 27, 527-545.

[4] Yuan, Y., Amine, K., Lu, J., Shahbazian-Yassar, R., Understanding materials challenges for rechargeable ion batteries with in situ transmission electron microscopy. Nat. Commun. 2017, 8, 1-14.

[5] Nitta, N., Wu, F., Lee, J. T., Yushin, G., Li-ion battery materials: Present and future. Mater. Today 2015, 18, 252-264.

[6] Zhu, Z., Kan, R., Hu, S., He, L., Hong, X., Tang, H., Luo, W., Recent Advances in HighPerformance Microbatteries: Construction, Application, and Perspective. Small 2020, 16, 2003251.

[7] Manthiram, A., Yu, X., Wang, S., Lithium battery chemistries enabled by solid-state electrolytes. Nat. Rev. Mater. 2017, 2, 1-16.

[8] Bharathi, K. K., Tan, H., Takeuchi, S., Meshi, L., Shen, H., Shin, J., Takeuchi, I., Bendersky, L. A., Effect of oxygen pressure on structure and ionic conductivity of epitaxial $\mathrm{Li}_{0.33} \mathrm{La}_{0.55} \mathrm{TiO}_{3}$ solid electrolyte thin films produced by pulsed laser deposition. 
RSC Adv. 2016, 6, 61974-61983.

[9] Kim, K. H., Iriyama, Y., Yamamoto, K., Kumazaki, S., Asaka, T., Tanabe, K., Fisher, C. A. J., Hirayama, T., Murugan, R., Ogumi, Z., Characterization of the interface between $\mathrm{LiCoO}_{2}$ and $\mathrm{Li}_{7} \mathrm{La}_{3} \mathrm{Zr}_{2} \mathrm{O}_{12}$ in an all-solid-state rechargeable lithium battery. J. Power Sources 2011, 196, 764-767.

[10] Long, J. W., Dunn, B., Rolison, D. R., White, H. S., Three-dimensional battery architectures. Chem. Rev. 2004, 104, 4463-4492.

[11] Oudenhoven, J. F. M., Baggetto, L., Notten, P. H. L., All-solid-state lithium-ion microbatteries: A review of various three-dimensional concepts. Adv. Energy Mater. $2011,1,10-33$.

[12] Yue, C., Li, J., Lin, L., Fabrication of Si-based three-dimensional microbatteries: A review. Front. Mech. Eng. 2017, 12, 459-476.

[13] Ferrari, S., Loveridge, M., Beattie, S. D., Jahn, M., Dashwood, R. J., Bhagat, R., Latest advances in the manufacturing of 3D rechargeable lithium microbatteries. J. Power Sources 2015, 286, 25-46.

[14] Bruce, P. G., Martinet, S., Nanomaterials for rechargeable lithium batteries. Nanosci. Technol. 2016, 471-512.

[15] Thostenson, E. T., Li, C., Chou, T. W., Nanocomposites in context. Compos. Sci. Technol. 2005, 65, 491-516.

[16] Palmero, P., Structural Ceramic Nanocomposites: A Review of Properties and Powders' Synthesis Methods. Nanomaterials 2015, 5, 656-696.

[17] Stuart, M. A. C., Huck, W. T. S., Genzer, J., Müller, M., Ober, C., Stamm, M., Sukhorukov, G. B., Szleifer, I., Tsukruk, V. V, Urban, M., Winnik, F., Zauscher, S., Luzinov, I., Minko, S., Emerging applications of stimuli-responsive polymer materials. Nat. Mater. 2010, 9, 101-13.

[18] Imada, M., Fujimori, A., Tokura, Y., Metal-insulator transitions. Rev. Mod. Phys. 1998, 70, 1039-1263.

[19] Elbio, D., Complexity in Strongly Correlated Electronic Systems. Science 2005, 309, $257-262$.

[20] Zhang, W., Ramesh, R., MacManus-Driscoll, J. L., Wang, H., Multifunctional, selfassembled oxide nanocomposite thin films and devices. MRS Bull. 2015, 40, 736-745.

[21] Huang, J., MacManus-Driscoll, J. L., Wang, H., New epitaxy paradigm in epitaxial selfassembled oxide vertically aligned nanocomposite thin films. J. Mater. Res. 2017, 32, 4054-4066.

[22] Zheng, H., Wang, J., Lofland, S. E., Ma, Z., Mohaddes-Ardabili, L., Zhao, T., Salamanca-Riba, L., Shinde, S. R., Ogale, S. B., Bai, F., Viehland, D., Jia, Y., Schlom, D. G., Wuttig, M., Roytburd, A., Ramesh, R., Multiferroic $\mathrm{BaTiO}_{3}-\mathrm{CoFe}_{2} \mathrm{O}_{4}$ Nanostructures. Science 2004, 303, 661-663.

[23] Harrington, S. A., Zhai, J., Denev, S., Gopalan, V., Wang, H., Bi, Z., Redfern, S. A. T., Baek, S. H., Bark, C. W., Eom, C. B., Jia, Q., Vickers, M. E., MacManus-Driscoll, J. L., Thick lead-free ferroelectric films with high Curie temperatures through nanocomposite- 
induced strain. Nat. Nanotechnol. 2011, 6, 491-495.

[24] Wang, Z., Li, Y., Viswan, R., Hu, B., Harris, V. G., Li, J., Viehland, D., Engineered magnetic shape anisotropy in $\mathrm{BiFeO}_{3}-\mathrm{CoFe}_{2} \mathrm{O}_{4}$ self-assembled thin films. ACS Nano $2013,7,3447-3456$.

[25] Chen, A., Bi, Z., Tsai, C. F., Lee, J., Su, Q., Zhang, X., Jia, Q., MacManus-Driscoll, J. L., Wang, H., Tunable low-field magnetoresistance in $\left(\mathrm{La}_{0.7} \mathrm{Sr}_{0.3} \mathrm{MnO}_{3}\right)_{0.5}:(\mathrm{ZnO})_{0.5}$ selfassembled vertically aligned nanocomposite thin films. Adv. Funct. Mater. 2011, 21, $2423-2429$.

[26] Hsieh, Y. H., Liou, J. M., Huang, B. C., Liang, C. W., He, Q., Zhan, Q., Chiu, Y. P., Chen, Y. C., Chu, Y. H., Local conduction at the $\mathrm{BiFeO}_{3}-\mathrm{CoFe}_{2} \mathrm{O}_{4}$ tubular oxide interface. Adv. Mater. 2012, 24, 4564-4568.

[27] Lee, O., Harrington, S. A., Kursumovic, A., Defay, E., Wang, H., Bi, Z., Tsai, C. F., Yan, L., Jia, Q., MacManus-Driscoll, J. L., Extremely high tunability and low loss in nanoscaffold ferroelectric films. Nano Lett. 2012, 12, 4311-4317.

[28] Stratulat, S. M., Lu, X., Morelli, A., Hesse, D., Erfurth, W., Alexe, M., Nucleationinduced self-assembly of multiferroic $\mathrm{BiFeO}_{3}-\mathrm{CoFe}_{2} \mathrm{O}_{4}$ nanocomposites. Nano Lett. 2013, 13, 3884-3889.

[29] Ke, H., Zhang, H., Zhou, J., Jia, D., Zhou, Y., Room-temperature multiferroic and magnetodielectric properties of $\mathrm{SrTiO}_{3} / \mathrm{NiFe}_{2} \mathrm{O}_{4}$ composite ceramics. Ceram. Int. 2019, 45.

[30] Lee, S., MacManus-Driscoll, J. L., Research Update: Fast and tunable nanoionics in vertically aligned nanostructured films. APL Mater. 2017, 5, 042304.

[31] Ichino, Y., Yoshida, Y., Miura, S., Three-dimensional Monte Carlo simulation of nanorod self-organization in $\mathrm{REBa}_{2} \mathrm{Cu}_{3} \mathrm{O}_{\mathrm{y}}$ thin films grown by vapor phase epitaxy. Jpn. J. Appl. Phys. 2017, 56, 015601.

[32] Hennes, M., Schuler, V., Weng, X., Buchwald, J., Demaille, D., Zheng, Y., Vidal, F., Growth of vertically aligned nanowires in metal-oxide nanocomposites: Kinetic MonteCarlo modeling: Versus experiments. Nanoscale 2018, 10, 7666-7675.

[33] Hsieh, Y.-H. H., Strelcov, E., Liou, J.-M. M., Shen, C.-Y. Y., Chen, Y.-C. C., Kalinin, S. V., Chu, Y.-H. H., Electrical Modulation of the Local Conduction at Oxide Tubular Interfaces. ACS Nano 2013, 7, 8627-8633.

[34] Strelcov, E., Kim, Y., Jesse, S., Cao, Y., Ivanov, I. N., Kravchenko, I. I., Wang, C.-H. H., Teng, Y.-C. C., Chen, L.-Q. Q., Chu, Y. H., Kalinin, S. V., Probing Local Ionic Dynamics in Functional Oxides at the Nanoscale. Nano Lett. 2013, 13, 3455-3462. 


\title{
Chapter 2
}

\section{The building blocks of an All-Solid- State battery}

\begin{abstract}
Rechargeable batteries are among the most successful technologies for reversible and efficient electrochemical energy storage and conversion. The performance of rechargeable batteries depends essentially on the thermodynamics and kinetics of the electrochemical reactions involved in the components (i.e. anode, cathode and electrolyte) of the cell. Common rechargeable batteries are based on a liquid electrolyte, which implies that there are several restrictions for their design and size due to the available separators and liquid electrolytes. The liquid electrolyte also carry the inherent risk of leakage, limiting its application and miniaturization. Therefore, the need to implement solid-state batteries emerge. While most studies in battery materials are performed in bulk, thin film technology provides exclusive control over the crystallographic properties of the materials. Detailed understanding of the electrochemical behavior of specific crystal facets of battery materials can only be obtained when a single type of crystal orientation interfacing the electrolyte can be synthesized. This crucial requirement can be achieved by epitaxial thin film technology, in which the flat surface and restricted lattice plane of the thin film cathode simplify the reaction mechanism at such highly ordered cathode-electrolyte interface. Therefore, epitaxial engineering was applied, through Pulsed Laser Deposition, as a tool to study the relation between crystal orientation and ionic and electronic conduction, and to obtain improved control over the electrochemical properties of the $\mathrm{Li}_{4} \mathrm{Ti}_{5} \mathrm{O}_{12}$ (anode), $\mathrm{LiMn}_{2} \mathrm{O}_{4}$ (cathode) and $\mathrm{Li}_{0.5} \mathrm{La}_{0.5} \mathrm{TiO}_{3}$ (electrolyte) thin films, which could not be obtained in single crystals or polycrystalline samples.
\end{abstract}

Based on the publications:

D.M. Cunha, T.A. Hendriks, A. Vasileiadis, C.M. Vos, T. Verhallen, D.P. Singh, M. Wagemaker, M. Huijben, Doubling Reversible Capacities in Epitaxial $\mathrm{Li}_{4} \mathrm{Ti}_{5} \mathrm{O}_{12}$ Thin Film Anodes for Microbatteries. ACS Appl. Energy Mater. 2019, 2, 5, 3410-3418.

T.A. Hendriks, D.M. Cunha, D.P. Singh, M. Huijben, Enhanced Lithium Transport by Control of Crystal Orientation in Spinel $\mathrm{LiMn}_{2} \mathrm{O}_{4}$ Thin Film Cathodes. ACS Appl. Energy Mater. 2018, 1, 12, 7046-7051. 


\subsection{Introduction}

Rechargeable batteries are among the most successful technologies for reversible and efficient electrochemical energy storage and conversion.[1] The performance of rechargeable batteries depends essentially on the thermodynamics and kinetics of the electrochemical reactions involved in the components (i.e. anode, cathode and electrolyte) of the cell. The electrochemical process is a redox reaction involving electrochemical charge transfer coupled with insertion/extraction of mobile guest ions into/from the structure of an electronic and ionic conductive host. During the past decade, extensive efforts have been dedicated to develop advanced batteries with large capacity, high energy and power density, high safety, long cycle-life, fast response and low cost.[2,3]

Common rechargeable batteries are based on a liquid electrolyte, which implies that there are several restrictions for their design and size due to the available separators and liquid electrolytes. The liquid electrolyte also carry the inherent risk of leakage, limiting its application and miniaturization. Therefore, the need to implement solid-state batteries emerge, facilitating miniaturization and creating more flexibility in the design of standalone microelectronic devices, enhancing their applicability, for example in medical implants, due to the avoided leakage risks. However, the successful application of allsolid-state microbatteries depends strongly on the enhancement of energy density and lifetime. While most studies in battery materials are performed in bulk, thin film technology remains a relatively unknown field in battery application. Hence, epitaxial engineering was applied as a tool to obtain improved control over the electrochemical properties of the thin films, which is unique for this system and cannot be obtained in single crystals or polycrystalline samples.

The most promising candidates of spinel electrode materials are $\mathrm{Li}_{4} \mathrm{Ti}_{5} \mathrm{O}_{12}$ (LTO) and $\mathrm{LiMn}_{2} \mathrm{O}_{4}$ (LMO) for anode and cathode application, respectively. LTO is a lithium intercalation compound exhibiting a theoretical capacity of $175 \mathrm{mAh} \cdot \mathrm{g}^{-1}$ with a flat insertion/extraction voltage of approximately $1.55 \mathrm{~V}$ versus $\mathrm{Li} / \mathrm{Li}^{+}$, well above the potential for the formation of dendritic lithium and for the formation of a solid-electrolyte interphase (SEI) from the reduction of the organic electrolyte.[4] This advantage comes with compromise of a lower overall battery voltage decreasing energy density as compared to graphite anodes.[5,6] On the cathode side, LMO is being studied as an alternative for commercial $\mathrm{LiCoO}_{2}$ (LCO),[2,7] for its relatively high operating voltage (4.1 $\mathrm{V}$ versus $\mathrm{Li} / \mathrm{Li}^{+}$) and comparable energy density (theoretically $148 \mathrm{mAh} \cdot \mathrm{g}^{-1}$ ) combined with low cost and no direct environmental or safety hazards.[8,9] Compared to liquid electrolytes, solid electrolytes have lower ionic conductivity, focusing research mostly on developing better ionic conductors.[10,11] The perovskite $\mathrm{Li}_{0.5} \mathrm{La}_{0.5} \mathrm{TiO}_{3}$ is a 
very promising solid-state electrolyte, showing high ionic conductivities $\left(\sim 10^{-3} \mathrm{~S} \cdot \mathrm{cm}^{-1}\right)$ at room temperature close to that of a standard liquid electrolytes (e.g. $1 \mathrm{M} \mathrm{LiPF}_{6} \mathrm{EC}: \mathrm{DMC}$ $\left.1: 1,10^{-2} \mathrm{~S} \cdot \mathrm{cm}^{-1}\right) \cdot[11,12]$

\subsection{Methods}

All samples presented in this chapter were grown by PLD on various single crystal Nbdoped (0.5 wt\%) $\mathrm{SrTiO}_{3}$ (STO) substrates with different crystal orientations ((100), (110) and (111)). The growth parameters used for the different materials are summarized in Table 2-1.

The structural parameters of the films were analyzed using X-ray diffraction (XRD) measurements, performed on a PANalytical X'Pert PRO diffractometer, with a PIXcel1D detector and a $\mathrm{Cu}$ source $(\lambda=1.5406 \AA)$. The surface morphology was studied using Tapping-mode atomic force microscopy (AFM), carried out in air on a Bruker ICON Dimension Microscope. Cross-sectional images and qualitative compositional analysis were done with a Zeiss Merlin high-resolution scanning electron microscopy (HRSEM).

For electrochemical characterization the films were transferred to an argon atmosphere glovebox $\left(<0.1 \mathrm{ppm}\right.$ of $\mathrm{H}_{2} \mathrm{O}$ and $\left.\mathrm{O}_{2}\right)$ and placed on a hot plate for $\sim 10 \mathrm{~min}$ at $125^{\circ} \mathrm{C}$ to remove any water content. Subsequently, they were positioned in an electrochemical ECref cell by EL-CELL and combined with a glass fiber separator of $1 \mathrm{~mm}$ thickness, 0.6 $\mathrm{mL}$ electrolyte with $1 \mathrm{M} \mathrm{LiPF}_{6}$ in 1:1 ethylene carbonate dimethyl carbonate (EC:DMC) and lithium metal anode. The electrochemical measurements were performed at $22{ }^{\circ} \mathrm{C}$ using a BioLogic VMP-300 system in a two-electrode setup. Since the voltage range and current applied varies for every material, further details will be given on the designated sections.

Table 2-1. Parameters for deposition of the different battery materials studied in this chapter.

\begin{tabular}{llll}
\hline & \multicolumn{1}{c}{ LMO } & \multicolumn{1}{c}{ LLTO } & \multicolumn{1}{c}{ LTO } \\
\hline Temperature $\left({ }^{\circ} \mathrm{C}\right)$ & 600 & 900 & 700 \\
Laser frequency $(\mathrm{Hz})$ & 2 & 20 & 5 \\
$\mathrm{O}_{2}$ Pressure $(\mathrm{mbar})$ & 0.13 & 0.2 & 0.2 \\
Thickness $(\mathrm{nm})$ & 110 & 110,450 & $55-330$ \\
Fluency $\left(\mathrm{J} \cdot \mathrm{cm}^{-2}\right)$ & 2.33 & 2.33 & 2.33 \\
Spot size $\left(\mathrm{mm}^{2}\right)$ & 1.92 & 1.92 & 1.92 \\
\hline
\end{tabular}




\subsection{Orientation-dependent intercalation kinetics for $\mathrm{Li}_{4} \mathrm{Ti}_{5} \mathrm{O}_{12}$ anode thin films}

The spinel $\mathrm{Li}_{4} \mathrm{Ti}_{5} \mathrm{O}_{12}$ (LTO) material has been extensively studied as an alternative to carbon anode materials in particular when cycle life and power density matters, because of its negligible volume change $(0.2-0.3 \%)$, high rate capability, good safety characteristics and high cycling stability.[4-6,13] Although the native LTO exhibits low electronic conductivity $\left(\sim 10^{-8}\right.$ to $\left.\sim 10^{-13} \mathrm{~S} \cdot \mathrm{cm}^{-1}\right)$ and low lithium-ion diffusion coefficient $\left(\sim 10^{-9}\right.$ to $\left.\sim 10^{-16} \mathrm{~cm}^{2} \cdot \mathrm{s}^{-1}\right)$, considerable research on the morphological and surface optimization, doping, and nanostructuring has dramatically improved its capacity and rate capability.[4,13] Despite these advantageous properties, application of LTO is limited by a higher operating voltage and a lower capacity as compared to existing graphite anode.

(a) $\mathrm{Li}_{4} \mathrm{Ti}_{5} \mathrm{O}_{12}$

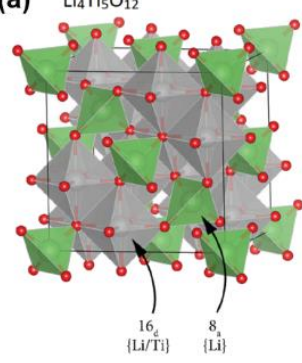

(b) $\mathrm{Li}_{7} \mathrm{Ti}_{5} \mathrm{O}_{12}$

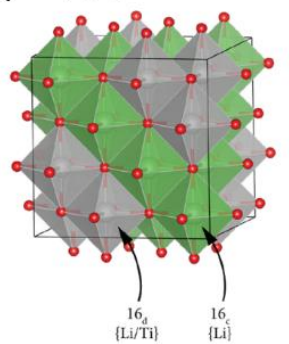

(c)

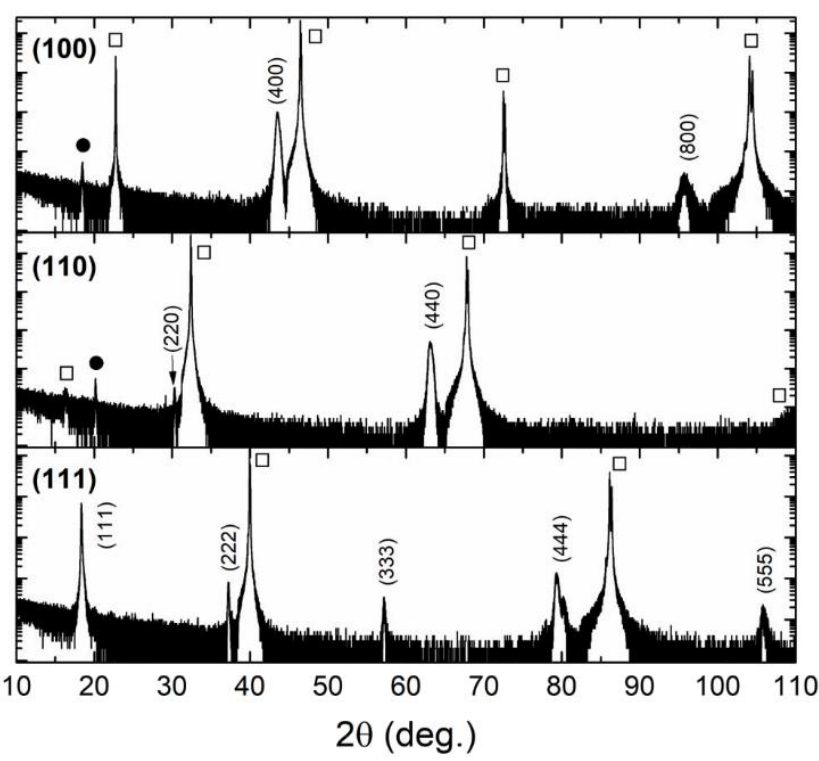

Figure 2-1. Schematics of the crystal structures of (a) spinel $\mathrm{Li}_{4} \mathrm{Ti}_{5} \mathrm{O}_{12}$ and (b) rock-salt $\mathrm{Li}_{7} \mathrm{Ti}_{5} \mathrm{O}_{12}$. (c) Out-of-plane XRD measurements of $220 \mathrm{~nm} \mathrm{Li} 4 \mathrm{Ti}_{5} \mathrm{O}_{12}$ thin films on $\mathrm{Nb}: \mathrm{SrTiO}_{3}$ substrates with different crystal orientations: (100), (110) and (111). Peaks of the $\mathrm{Nb}: \mathrm{SrTiO}_{3}$ substrates are indicated by $\square$, while minor contributions of $\beta-\mathrm{Li}_{2} \mathrm{TiO}_{3}$ are given by

Optimal performance of LTO relies on a fundamental understanding of the lithium diffusion kinetics and the underlying phase transformation mechanism. The defective spinel $\mathrm{Li}_{4} \mathrm{Ti}_{5} \mathrm{O}_{12}$ can be indexed by the space group of $\mathrm{Fd} \overline{3} \mathrm{~m}(a=8.36 \AA$ ), see Figure 
2-1a, in which tetrahedral 8 a sites are occupied by $\mathrm{Li}^{+}$, and octahedral $16 \mathrm{~d}$ sites are occupied by $\mathrm{Li}^{+}$and $\mathrm{Ti}^{4+}$ randomly in a ratio of $\mathrm{Li} / \mathrm{Ti}=1 / 5$, while the octahedral $32 \mathrm{e}$ sites are taken by $\mathrm{O}^{2-}$. Therefore, $\mathrm{Li}_{4} \mathrm{Ti}_{5} \mathrm{O}_{12}$ can be represented as $\left[\mathrm{Li}_{3}\right]^{8 \mathrm{a}}\left[\mathrm{LiTi}_{5}\right]^{16 \mathrm{~d}}\left[\mathrm{O}_{12}\right]^{32 \mathrm{e}}$. Lithiation leads to occupation of all the octahedral 16c sites and emptying of the tetrahedral $8 \mathrm{a}$ sites to obtain the rock-salt-structured $\mathrm{Li}_{7} \mathrm{Ti}_{5} \mathrm{O}_{12}$, which can be represented as $\left[\mathrm{Li}_{6}\right]^{16 \mathrm{c}}\left[\mathrm{LiTi}_{5}\right]^{16 \mathrm{~d}}\left[\mathrm{O}_{12}\right]^{32 \mathrm{e}}$, see Figure 2-1b. Although the lithiation process was for a long time understood as a two-phase reaction where the two end members coexist during lithium insertion/extraction, recent studies have demonstrated that a solid-solution exists with both $\mathrm{Li}_{4} \mathrm{Ti}_{5} \mathrm{O}_{12}$ and $\mathrm{Li}_{7} \mathrm{Ti}_{5} \mathrm{O}_{12}$ intimately mixed at nanometer length scales.[14-16] Furthermore, lithium compositions exceeding $\mathrm{Li}_{7} \mathrm{Ti}_{5} \mathrm{O}_{12}$ have been observed during the first cycles experimentally [17-20] as well as theoretically,[21,22] indicating this to be a surface-related phenomenon.

\subsubsection{Control over the LTO crystal orientation}

To obtain improved control over the electrochemical properties of $\mathrm{Li}_{4} \mathrm{Ti}_{5} \mathrm{O}_{12}$ thin films epitaxial engineering was applied, which cannot be obtained in single crystals or polycrystalline samples. To study the relation between crystal structure and ionic and electronic conduction, $\mathrm{Li}_{4} \mathrm{Ti}_{5} \mathrm{O}_{12}$ thin films were grown by PLD on various single crystal $\mathrm{Nb}$-doped (0.5 wt\%) $\mathrm{SrTiO}_{3}$ (STO) substrates with different crystal orientations ((100), (110) and (111)). All LTO thin films were deposited under the same conditions for various thicknesses in the range of $55-330 \mathrm{~nm}$.

The structural quality of the LTO films was investigated by XRD analysis, as shown in Figure 2-1c. The three types of LTO films grown on Nb-doped STO substrates with different orientations exhibit coherent growth in which the out-of-plane crystal orientation of the films is aligned with the orientation of the substrate. The LTO(111) films show the presence of a highly crystalline epitaxial layer, with a lattice parameter of $\sim 8.32 \AA$, without any impurity phase, in good agreement with previous study of LTO growth on STO(111) substrates.[19,23] This suggests that the PLD deposition process parameters (e.g. temperature, pressure, laser energy density, target composition) were optimized successfully to correct for any loss of volatile lithium during ablation, nucleation or growth. Interestingly, the LTO films with (100) and (110) orientations still show minor contributions of a secondary phase, although all three types of LTO films were grown during the same deposition procedure. The extra peaks at low diffraction angles cannot be ascribed to anatase or rutile $\mathrm{TiO}_{2}$ as observed in previous studies, $[19,20]$ but suggest the presence of a small amount of monoclinic $\beta-\mathrm{Li}_{2} \mathrm{TiO}_{3}$ with respectively (002) or (110) orientation.[24] Although $\beta$ - $\mathrm{Li}_{2} \mathrm{TiO}_{3}$ has also been investigated as anode material,[25] it will have a negligible effect on the electrochemical performance of the LTO thin films, as the anodic reduction and cathodic oxidation reactions in $\beta-\mathrm{Li}_{2} \mathrm{TiO}_{3}$ 
take place below $\sim 0.8 \mathrm{~V}$.[25] This is far below the potential window in the measurements of $1.0-2.5 \mathrm{~V}$ performed here.

The alignment of the out-of-plane crystal orientation for all types of LTO films, suggests an epitaxial relation between the crystal structures of the deposited LTO films and the underlying STO substrates, although large differences exist between spinel LTO $(a=8.36 \AA)$ and perovskite STO $(a=3.91 \AA)$. Therefore, the in-plane crystal orientations of the LTO films were studied by XRD $\varphi$-scans along the LTO/STO directions of respectively $(440) /(110),(111) /(111)$ and $(440) /(110)$, see Figure $2-2$. The separation of the STO peaks in the (100), (110) and (111) plane by respectively $90^{\circ}, 180^{\circ}$ and $120^{\circ}$ are consistent with the perovskite crystal structure, shown in the insets of Figure 2-2. For the LTO(100) and LTO(110) films the in-plane peaks are at the same angles as the substrate orientation indicating the in-plane alignment of the LTO layer to the STO structure. For the LTO(111) films the presence of two domains can be observed, in which part of the LTO layer is in-plane aligned to the STO substrate but the majority is rotated $60^{\circ}$ in-plane with respect to the substrate.
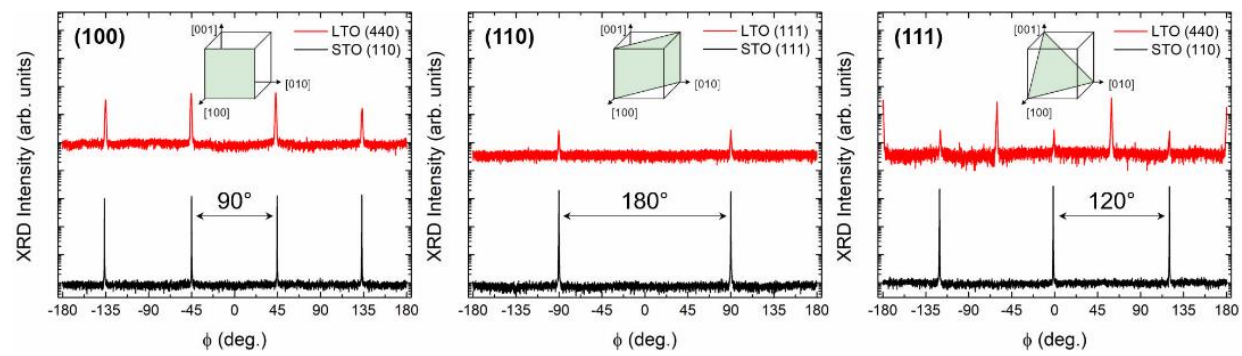

Figure 2-2. In-plane XRD measurements of $220 \mathrm{~nm} \mathrm{Li}_{4} \mathrm{Ti}_{5} \mathrm{O}_{12}$ thin films on $\mathrm{Nb}: \mathrm{SrTiO}_{3}$ substrates with different crystal orientations: (100), (110) and (111). Peaks of the $\mathrm{Li}_{4} \mathrm{Ti}_{5} \mathrm{O}_{12}$ thin films and $\mathrm{Nb}: \mathrm{SrTiO}_{3}$ substrates are indicated respectively in red and black.

The observed preferred orientation of the LTO films was confirmed by detailed analysis of the surface morphology through atomic force microscopy (AFM), see Figure 2-3. The surface of the LTO(100) film exhibits square-like structures with significant height differences $(\mathrm{Rms}=\sim 12.7 \mathrm{~nm})$, which is in good agreement with previously observed octahedron spinel structures.[26,27] Such pyramidal spinel structures consist of $\langle 111\rangle$ crystal facets on all four sides with an occasional presence of a truncated top of the pyramid exhibiting a $\langle 100\rangle$ crystal facet. These square-like structures confirm the $90^{\circ}$ periodicity in the in-plane orientation as observed in XRD measurements, see Figure 2-2. The LTO(110) film forms a layer with rooftop-like structures and a lower surface roughness $(\mathrm{Rms}=\sim 8.2 \mathrm{~nm})$, caused by the anisotropic nature of the (110)-plane which favors diffusion of atoms along the [110]-direction as compared to the [001]direction.[28] This results in elongated $\langle 111\rangle$ crystal facets exposed on the surface, which are all aligned in the same direction in good agreement with the $180^{\circ}$ periodicity observed in XRD results. Finally, the LTO(111) films form a layer with triangle-like structures 
exhibiting a very low surface roughness $(\mathrm{Rms}=\sim 1.4 \mathrm{~nm})$. The triangular shape corresponds to the (111) plane in a cubic structure and matches the observed $120^{\circ}$ periodicity in the XRD measurements. Furthermore, two different types of in-plane triangle orientations can be observed, which confirms the presence of two domain types, rotated $60^{\circ}$ with respect to each other, as observed in XRD analysis.
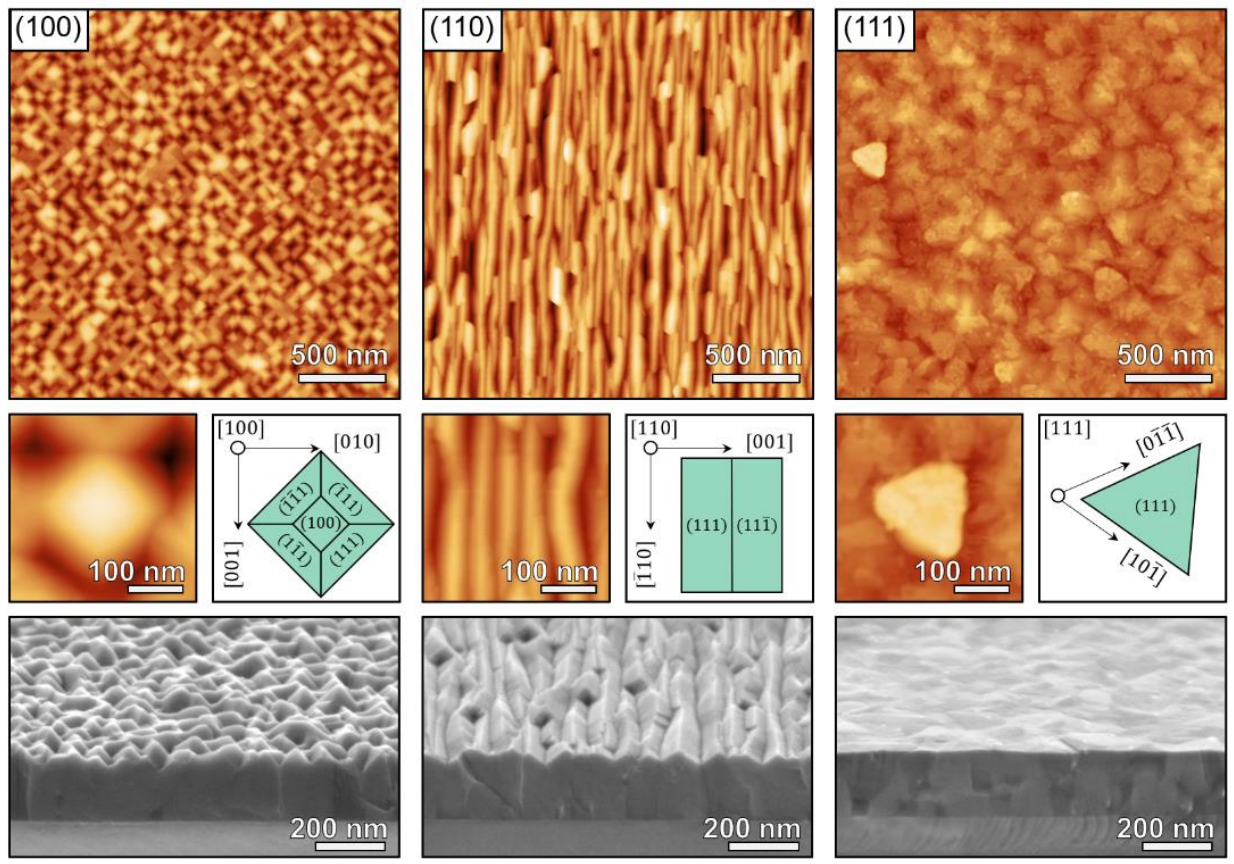

Figure 2-3. AFM (top) and SEM (bottom) analysis of the surface morphology of $220 \mathrm{~nm}$ $\mathrm{Li}_{4} \mathrm{Ti}_{5} \mathrm{O}_{12}$ thin films on $\mathrm{Nb}: \mathrm{SrTiO}_{3}$ substrates with different crystal orientations: (100), (110) and (111). Schematics (middle) are shown of the expected crystal facets for the different surface morphologies.

Therefore, all three types of LTO films with different out-of-plane orientations ((100), (110) and (111)) exhibit surfaces exposing predominantly $\langle 111\rangle$ crystal facets, which indicates that this is the lowest energy state surface. This is in good agreement with previous theoretical [22] and experimental [26] studies on LTO crystals, which demonstrated that oxygen-terminated (110) and (111) facets exhibit surface energies about half of a (100) facet due to the minimal loss of coordination to the subsurface $\mathrm{TiO}_{6}$ octahedra, and much lower than lithium- or titanium-terminated surfaces. 


\subsubsection{Electrochemical behavior of epitaxial LTO films}

The lithium intercalation characteristics were studied by galvanostatic chargedischarge analysis of electrochemical cells in which the LTO thin films were measured against lithium metal with a liquid electrolyte. To do so, the samples were cycled between 1.0 and $2.5 \mathrm{~V}$ with currents of $10,20,40$ and $100 \mu \mathrm{A}$, corresponding to $\mathrm{C}$-rates of approximately $3 \mathrm{C}, 6 \mathrm{C}, 12 \mathrm{C}$ and $30 \mathrm{C}$, respectively. A potentiostatic period of $5 \mathrm{~min}$. is used to ensure complete charge or discharge before the next step. Figure 2-4 shows charge-discharge curves from the first to the twentieth cycle for a constant current of 10 $\mu \mathrm{A}$, corresponding to a (dis)charge rate of $3 \mathrm{C}$, and cutoff voltages of 1.0 and $2.5 \mathrm{~V}$. A clear voltage plateau can be observed at $\sim 1.55 \mathrm{~V}$ in good agreement with bulk LTO characteristics. $[4,13]$ During the initial charge-discharge cycles of the LTO films the charge capacity remains constant, while the discharge capacity is reducing towards a constant level. As a result, the calculated Coulombic efficiency is changing within this initial cycling towards $\sim 100 \%$. However, when the LTO films are subsequently cycled at low rates of $3 \mathrm{C}$ within the voltage range between 1.0 and $2.5 \mathrm{~V}$ the discharge capacity is always larger than the charge capacity, corresponding to a Coulombic efficiency of about 95\%. This effect can also be observed in previous studies on epitaxial LTO thin films $[19,20,23]$ as well as on polycrystalline LTO thin films,[29] but was never discussed specifically.
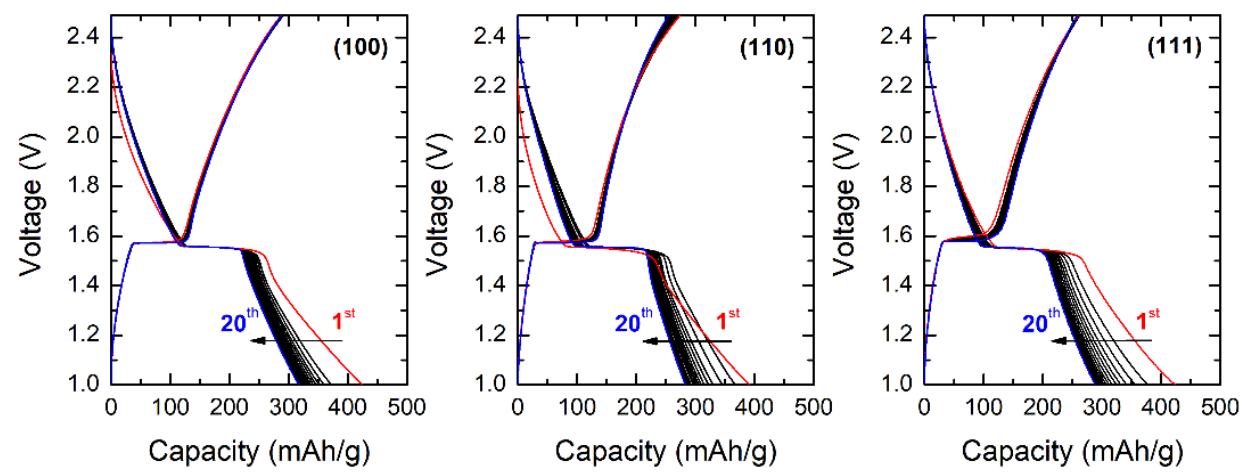

Figure 2-4. Charge-discharge analysis of the first 20 cycles on $220 \mathrm{~nm} \mathrm{Li}{ }_{4} \mathrm{Ti}_{5} \mathrm{O}_{12}$ thin films on $\mathrm{Nb}: \mathrm{SrTiO}_{3}$ substrates with different crystal orientations ((100), (110) and (111)). During the measurements a current of $10 \mu \mathrm{A}$ was used, which provided a (dis)charge rate of $3 \mathrm{C}$.

The LTO thin films do not exhibit an increase in internal resistance due to a growing SEI layer, but the experiments show a stable, reversible lithiation process. Therefore, it is suggested that the higher discharge capacity, as compared to the charge capacity, is due to a small increase in the SEI layer thickness which easily dissolves into the liquid 
electrolyte during subsequent charging. This process of SEI formation and dissolution at the LTO surface was previously demonstrated [30] and is more pronounced in LTO thin films due to the limited volume of the samples. Further research is required to achieve detailed understanding on the contribution of the SEI layer for lithium storage in the LTO thin films systems.

All three crystal orientations showed high electrochemical performance with good cyclability, as well as very high discharge capacities far above the theoretical capacity of $175 \mathrm{mAh} \cdot \mathrm{g}^{-1}$ for the $\mathrm{Li}_{7} \mathrm{Ti}_{5} \mathrm{O}_{12}$ composition where all octahedral $\mathrm{Li}$-positions are occupied. The total discharge capacity was the highest for the (100)-oriented LTO film, $\sim 313 \mathrm{mAh} \cdot \mathrm{g}^{-1}$, while the (110)- and (111)-oriented LTO films exhibit lower discharge capacities of respectively $\sim 277 \mathrm{mAh} \cdot \mathrm{g}^{-1}$ and $\sim 283 \mathrm{mAh} \cdot \mathrm{g}^{-1}$. The large surface area of the (100)-oriented LTO film, caused by pyramidal surface morphology, is suggested to cause the enhanced lithium storage as compared to the other crystal orientations. The crystal facets on all films are predominantly $\langle 111\rangle$, which eliminates any possible effect from local variations in crystal facets. Surpassing the theoretical capacity for $\mathrm{Li}_{7} \mathrm{Ti}_{5} \mathrm{O}_{12}$ composition is in good agreement with previous observations, although those theoretical models [21,22] and experimental studies on polycrystalline materials $[17,18]$ required a voltage range from $2.5 \mathrm{~V}$ to $0.01 \mathrm{~V}$ to realize lithiation up to $\mathrm{Li}_{8.5} \mathrm{Ti}_{5} \mathrm{O}_{12}$ exhibiting capacities of $\sim 260 \mathrm{mAh} \cdot \mathrm{g}^{-1}$. Here, reversible high capacities of $\sim 280-310 \mathrm{mAh} \cdot \mathrm{g}^{-1}$ were achieved for epitaxial films in the limited voltage range between $2.5 \mathrm{~V}$ and $1.0 \mathrm{~V}$, confirming the enhanced storage of lithium at the $\langle 111\rangle$ facets.[22] Interestingly, the results show enhanced lithiation for all three film orientations ((100), (110) and (111)), all exhibiting $\langle 111\rangle$ facets, in strong contrast to previous limitation to the (111) orientation.[19]

\subsubsection{Film thickness dependence on electrochemistry}

To distinguish the surface contribution from the bulk LTO layer dependent intercalation processes, variations in the electrochemical behavior were investigated for different LTO layer thicknesses. Figure 2-5 shows the charge-discharge curves for LTO film thicknesses in the range $55-330 \mathrm{~nm}$ together with the total capacity for each cell after charging or discharging. For the full thickness range and for all orientations the LTO films exhibit good electrochemical behavior with clear voltage plateaus as well as significant tails above and below those plateaus. The limited volume of the LTO layer in the thin films causes the surface contributions in the tails to be more pronounced as compared to bulk studies. It can be observed that for all three orientations the total capacities of the LTO films increase linearly with thickness, suggesting the presence of a volume dependent capacity in combination with a constant surface capacity. The linear fits indicate similar volume dependent discharge capacities of $\sim 120 \mathrm{mAh} \cdot \mathrm{g}^{-1}$ for all 
orientations, while minimal variations can be observed for the offset on the capacity axes for the three orientations. These fits suggest the presence of a constant surface capacity of $\sim 2 \mu \mathrm{Ah}\left(8 \mu \mathrm{Ah} \cdot \mathrm{cm}^{-2}\right)$ for all three orientations. This extra capacity cannot be completely stored within the LTO layer, as it would require an extra $\mathrm{Li}_{7} \mathrm{Ti}_{5} \mathrm{O}_{12}$ layer with a thickness of about $130 \mathrm{~nm}$. Therefore, the surface capacity can only partially be realized in an LTO layer with a higher lithiation level (i.e. $\mathrm{Li}_{9} \mathrm{Ti}_{5} \mathrm{O}_{12}$ ). However, the SEI was recently suggested to act as an extra charge reservoir with a significant contribution to the reversible lithiation process.[31] The combination of both volume and surface contributions explains the measured large capacities in thin films (see Figure 2-4), and strongly points out the necessity to distinguish between them instead of calculating a full volumetric capacity as was done in previous studies.
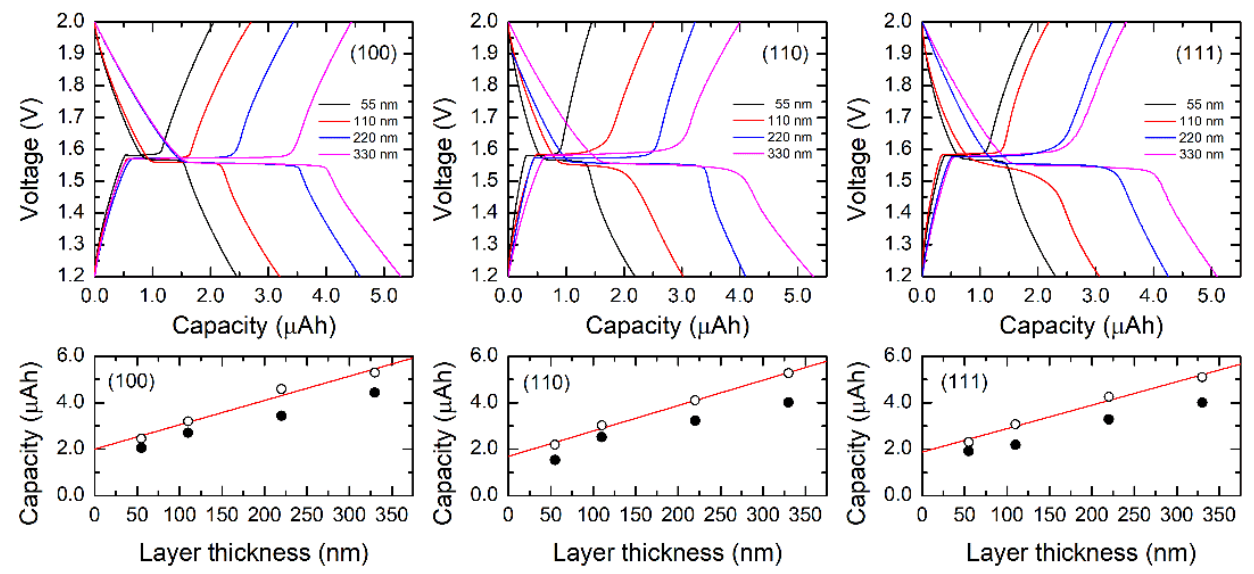

Figure 2-5. Layer thickness dependence of charge-discharge cycling (top) and total capacity (bottom) after charging (closed symbols) and after discharging (open symbols) of $\mathrm{Li}_{4} \mathrm{Ti}_{5} \mathrm{O}_{12}$ thin films on $\mathrm{Nb}: \mathrm{SrTiO}_{3}$ substrates with different crystal orientations ((100), (110) and (111)). During the measurements a current was provided to result in a (dis)charge rate of 3C. Linear fits are shown for the thickness dependent total discharge capacity.

The rate dependence of the discharge capacity is shown in more detail in Figure 2-6 for the LTO films with different crystal orientations. After the initial 20 charge-discharge cycles with $3 \mathrm{C}$ (i.e. current of $10 \mu \mathrm{A}$ ) the films are consecutively cycled at various rates in the range $3 \mathrm{C}-30 \mathrm{C}$ (i.e. currents $10-100 \mu \mathrm{A}$ ) before finishing the sequence with the final 60 cycles at $3 \mathrm{C}$. When the LTO films are cycled within the voltage range between 1.0 and $2.5 \mathrm{~V}$, the Coulombic efficiencies are very close to $100 \%$ for a high (dis)charge rate of $30 \mathrm{C}$ and a few percent lower for a slow (dis)charge rate of $3 \mathrm{C}$, as discussed before. The results show the stability of the LTO films during substantial cycling, as well as the enhanced performance of the (100)-oriented film as compared to the other orientations. However, the capacity taken over the full voltage range of $1.0-2.5 \mathrm{~V}$ contains the surface capacity together with the volumetric capacity, and the surface capacity is significantly 
larger for the (100)-oriented LTO film due the large surface area of the pyramidal morphology. The observed variation in surface area between the differently oriented films (about 30\% more surface area for (100)-oriented films as compared to (110)- and (111)oriented films, see Figure 2-3), explains this difference in capacity. When taking only the capacity at the voltage plateau $(1.5-1.6 \mathrm{~V})$ into account, the surface capacities do not contribute significantly and all three orientations show, at 3C, similar capacities of about $120 \mathrm{mAh} \cdot \mathrm{g}^{-1}$, in good agreement with the capacities determined from the layer thickness dependence.
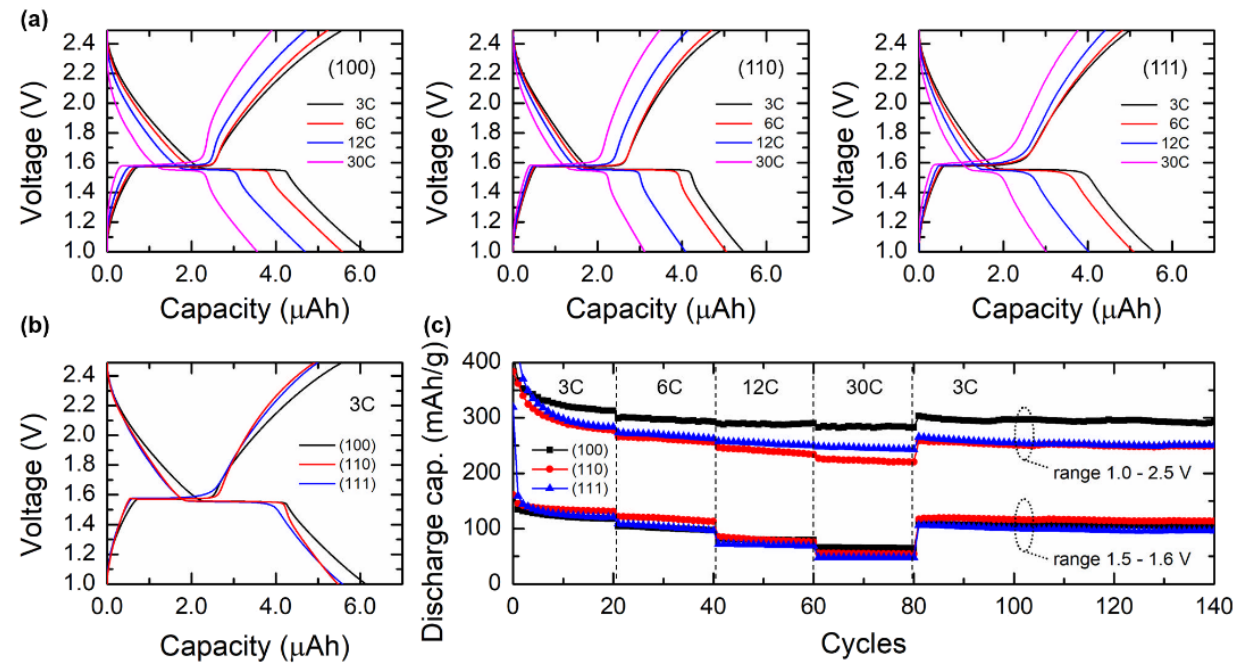

Figure 2-6. Charge-discharge rate dependence (a) of $220 \mathrm{~nm} \mathrm{Li} \mathrm{Ti}_{5} \mathrm{O}_{12}$ thin films on $\mathrm{Nb}: \mathrm{SrTiO}_{3}$ substrates with different crystal orientations ((100), (110) and (111)). During the measurements the current was varied between 10 and $100 \mu \mathrm{A}$, which led to a range of (dis)charge rates of 3C-30C. (b) Crystal orientation dependent charge-discharge analysis at a rate of 3C. (c) Cycle life analysis of the discharge capacity at various rates determined over the full voltage range of $1.0-2.5 \mathrm{~V}$ or only the voltage plateau between $1.5-1.6 \mathrm{~V}$. A potentiostatic period of $5 \mathrm{~min}$. is used to ensure complete charge or discharge before the next step.

At the highest rate of 30C the LTO films still exhibit volumetric capacities of about 60 $\mathrm{mAh} \cdot \mathrm{g}^{-1}$, in good agreement with values obtained for thick bulk LTO anodes.[32] The difference in measured capacities for the two voltage ranges suggest the presence of surface capacities of $\sim 15 \mu \mathrm{Ah} \cdot \mathrm{cm}^{-2}$ for (100)-oriented films and $\sim 12 \mu \mathrm{Ah} \cdot \mathrm{cm}^{-2}$ for (110)and (111)-oriented films. Furthermore, the measured surface capacities remain highly reversible with good cyclability up to those high (dis)charge rates. 


\subsubsection{Phase-field modelling of the lithiation mechanism}

In a collaborative work with Prof. Dr. Wagemaker from TU Delft, the lithiation mechanism of epitaxial LTO thin films was analyzed in detail by applying a phase-field model based on non-equilibrium electrochemical thermodynamics.[32] The model includes a thermodynamic description of the active material,[33-35] being able to capture phase separation in LTO electrodes and, coupled with a vacancy-based diffusion description in the solid, has been shown to correctly describe the material performance.[32] The model can only study Li-ion storage in the bulk and thus it is suitable to investigate the bulk capacities expected from the thin films, aiming to elucidate the possible limiting factors, including Li-ion diffusion, Li-ion transport, and electronic transport.

It is important to note that no thermodynamic or kinetic parameter was tuned to match the experimental results. All the parameters are identical to the ones reported in the prototypical case [29] and can be found somewhere else.[36] Regarding the geometrical features, the thin films are electrodes with practically zero porosity. In that sense, the surface of the thin film exposed to the electrolyte is expected to be completely wetted with no further Li-ion transport within the electrode due to the absence of pores. This indicates that the diffusion coordinate within the solid particles, mimicking the grains of the thin film, needs to be in the order of magnitude of the film thickness. The absence of pores also indicates that the lithiation wave should propagate in a single direction (vertical to the film), from the liquid electrolyte towards the Nb-doped STO current collector. However, the existence of multiple interconnected grains is evident in the SEM images (Figure 2-3) and complicates the solid description, where the introduction of more grain boundaries is likely to result in more $\mathrm{Li}_{4} \mathrm{Ti}_{5} \mathrm{O}_{12} / \mathrm{Li}_{7} \mathrm{Ti}_{5} \mathrm{O}_{12}$ phase interfaces that have been shown to catalyze Li-ion diffusion.[14,16] This is also supported by electrodes build-up by large secondary particles consisting of smaller primary particles showing superior electrochemical performance.[4,37,38] In the model, various particle shapes (spherical, cylindrical and single-direction rectangular) were investigated, that differ with regard to the volume fraction experienced and keep the Li-ion diffusion coordinate equal to the film thickness. Since all the other parameters were experimentally determined, the particle geometry that fits best the capacity trend as a function of current and film thickness was studied and conclusions regarding the effect of the interconnected grains, based on the different volume fractions, were drawn.

The simulation results of the simple spherical approximation are plotted and fitted with a shape preserving interpolant, creating the trend lines depicted in Figure 2-7a including the experimental results for comparison. The experimental values resulted from averaging the bulk $(1.5-1.6 \mathrm{~V})$ capacities of all three oriented LTO films ((100), (110) and (111)) at the respective rates and thicknesses. The simulated trend lines match well with the decreasing capacity "staircase" observed experimentally. Excellent agreement is 
observed for the thicker LTO films (220 and $330 \mathrm{~nm}$ ) while a larger capacity discrepancy is found for the thinner films where the simulated results converge faster to the theoretical maximum capacity. This is reasonable, as the ideal spherical approximation overestimates grain boundary diffusion (see Figure 2-7b) and the existence of larger errors in the measured capacity for thinner electrodes. However, the single-direction rectangular approximation, that initially may appear a realistic representation of the thin film batteries (see Figure 2-7c), predicts an extremely steep decrease in capacity with increasing thickness.

(a)

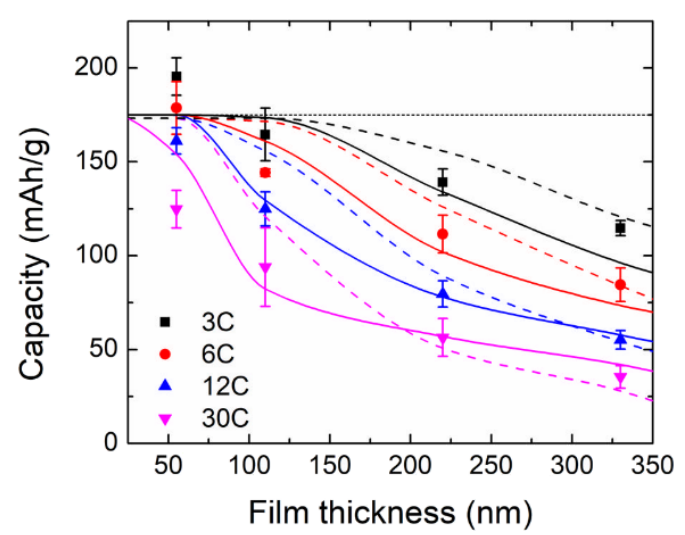

(b)

(d)

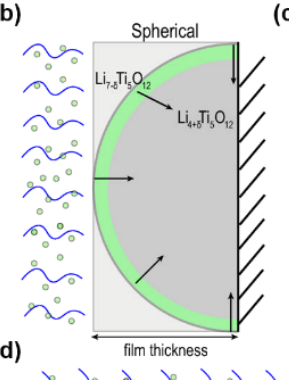

(c)
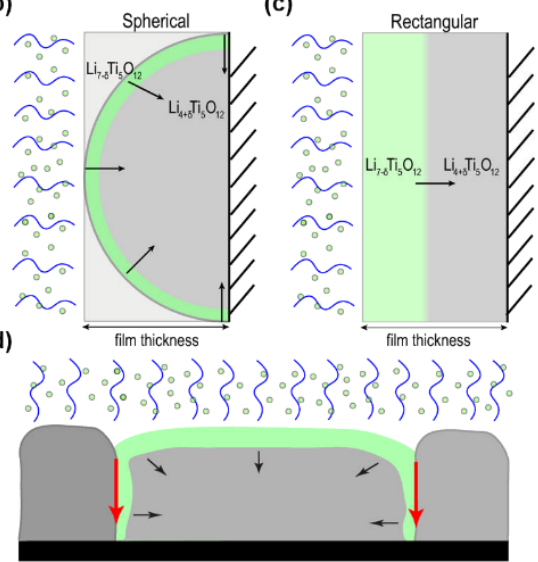

Figure 2-7. Lithiation mechanism in epitaxial $\mathrm{Li}_{4} \mathrm{Ti}_{5} \mathrm{O}_{12}$ thin films on $\mathrm{Nb}: \mathrm{SrTiO}_{3}$ substrates. (a) Gravimetric discharge capacity dependence on film thickness for different C-rates, determined at the voltage plateau (1.5 - 1.6 V). Experimental results (symbols) and theoretical phase field modeling for spherical approximation (dashed lines) and combined spherical and single-direction rectangular approximation (solid lines) are all shown. The two-phase lithiation model for (b) the spherical approximation, (c) singledirection rectangular approximation and (d) the mixed approximation, combining spherical and single-direction rectangular.

The results suggest that a mixed lithiation description, spherical and single-direction rectangular (Figure 2-7d), appears to be the best description of the experimental results for the thinnest LTO films (55 and $110 \mathrm{~nm}$ ), see Figure 2-7a. Thus, an exact multidimensional description of the grain geometry is required. This underlines the importance of understanding grain boundary diffusion and implies that the lithiation wave moves faster along the grain boundaries before moving inwards to the bulk of the grain, creating radial-like lithiation conditions for the bulk LTO. In this way the role of grain boundaries $[14,16]$ and the superior electrochemical behavior, encountered in literature among techniques utilizing large secondary particles, $[4,37,38]$ is rationalized. Thus, controlling the grain size is of great importance in order to tune the bulk capacity achievable for high C-rates in thin films. 
Analysis of the possible rate limiting kinetic mechanisms and their contribution to the total overpotential was performed.[36] Li-ion transport through the electrolyte, electronic transport, and Li-ion transfer over the electrolyte-electrode contact all exhibit minimal contributions to the internal resistance, and hence to the overpotentials that prevent utilizing the full capacity. The simulations confirm that the LTO thin films are limited completely by Li-ion diffusion in the solid phase, explaining the observed capacity loss with increasing current and thickness of material. Therefore, optimization of these batteries should focus on finding the optimum LTO thickness for the solid diffusion pathway or developing an intimate combination with a solid electrolyte with high ionic conductivity.

\subsection{Tailoring ionic transport by control of crystal orientation in spinel $\mathrm{LiMn}_{2} \mathrm{O}_{4}$ thin film cathode}

In the spinel cathode material $\mathrm{LiMn}_{2} \mathrm{O}_{4}$ (LMO), (space group $\mathrm{Fd} \overline{3} \mathrm{~m}$ ), $\mathrm{Li}$ and $\mathrm{Mn}$ occupy tetrahedral (8a) and octahedral (16d) sites in the intervening cubic close-packed array of oxygen atoms (32e sites). The edge-shared octahedral $\mathrm{Mn}_{2} \mathrm{O}_{4}$ host framework provides structural stability and interconnects face-shared tetrahedral lithium (8a) sites and empty octahedral (16c) sites. These interconnected pathways allow the threedimensional diffusion of lithium ions within the $\mathrm{Mn}_{2} \mathrm{O}_{4}$ framework, making $\mathrm{LiMn}_{2} \mathrm{O}_{4}$ suitable for high power application. The lithium (de)intercalation at (8a) tetrahedral sites results into the characteristic $\sim 4 \mathrm{~V}$ voltage plateau without distorting the spinel symmetry. Interestingly, this $\mathrm{Mn}_{2} \mathrm{O}_{4}$ framework can further host lithium into empty octahedral (16c) sites, resulting in a $3 \mathrm{~V}$ voltage plateau, almost doubling its capacity (theoretical capacity of $\mathrm{Li}_{2} \mathrm{Mn}_{2} \mathrm{O}_{4}$ is $285 \mathrm{mAh} \cdot \mathrm{g}^{-1}$ ) while undergoing a cubic to tetragonal phase transition. Furthermore, the operating voltage of $\mathrm{LiMn}_{2} \mathrm{O}_{4}$ can be increased to $\sim 5$ $\mathrm{V}$ by partially substituting $\mathrm{Mn}$ with $\mathrm{Ni}$ in the $\mathrm{Mn}_{2} \mathrm{O}_{4}$ framework.[39]

Despite of these advantageous properties, $\mathrm{LiMn}_{2} \mathrm{O}_{4}$ cathodes suffer from fading capacity and poor cycle life performance.[40] The origin of this capacity loss was attributed to two factors: first, the onset of Jahn-Teller distortion in deeply discharged electrodes,[41-43] and second, the dissolution of $\mathrm{Mn}$ ions from the $\mathrm{Mn}_{2} \mathrm{O}_{4}$ framework.[44] The Jahn-Teller distortion, accompanied by the cubic to tetragonal phase transition, irreversibly damages the structural integrity of the spinel framework during deep cycling down to $\sim 3 \mathrm{~V}$ causing capacity loss. However, the Jahn-Teller distortion can be avoided by limiting the charging and discharging to the $\sim 4 \mathrm{~V}$ plateau. Whereas, Mn dissolution causing continuous loss of active material and consequently blocking of 3D lithium diffusion pathways, hinders the overall cell performance and remains the key limitation for using $\mathrm{LiMn}_{2} \mathrm{O}_{4}$ cathodes.[45] Previous studies have suggested that acidification of electrolyte, caused by reaction of hexafluorophosphate $\left(\mathrm{LiPF}_{6}\right)$ salt in the 
electrolyte with $\mathrm{H}_{2} \mathrm{O}$, coupled with oxygen loss at the cathode surface, to be the origin of Mn dissolution.[44,46,47] The underlying mechanism can be understood via a disproportional reaction of $\mathrm{Mn}^{3+}$ generating soluble $\mathrm{Mn}^{2+}$ :

$$
4 \mathrm{H}^{+}+2 \mathrm{Li}\left(\mathrm{Mn}^{3+} \mathrm{Mn}^{4+}\right) \mathrm{O}_{4} \rightarrow 3 \lambda-\mathrm{Mn}^{4+} \mathrm{O} 2+\mathrm{Mn}^{2+}+2 \mathrm{Li}^{+}+2 \mathrm{H}_{2} \mathrm{O}
$$

Various strategies have been suggested to mitigate the Mn dissolution of $\mathrm{LiMn}_{2} \mathrm{O}_{4}$, such as aliovalent doping, surface coating, nanostructuring and mixed phase synthesis.[29,30-32,38,40-48] Although these strategies have indisputably shown significant enhancement in $\mathrm{LiMn}_{2} \mathrm{O}_{4}$ performance, it remains far from the desired level for usage in applications. Studies have shown that the specific crystal facet in contact with the electrolyte plays an important role in the electrochemical reactions occurring at the cathode surface for single crystalline nanowires, [57] truncated structures [58] and thin films.[59] It was concluded that, as the $\langle 111\rangle$ crystal facet possesses the lowest surface energy and the densest Mn atom arrangement, it can form a stable SEI layer and mitigate Mn dissolution, thus improving cycling stability. However, the (100)- and (110)-oriented facets were regarded to be better aligned to the lithium diffusion channels, thus able to increase discharge capacities and to facilitate high rate capabilities.[60]

Therefore, perfect control over the interface between the electrodes and electrolyte is needed but remains a great challenge. Most studies on $\mathrm{LiMn}_{2} \mathrm{O}_{4}$ thin films have investigated polycrystalline samples, while only limited experimental research has been performed on single crystalline thin films.[59,61-66] Characterization of epitaxial thin films has previously been focused on the structural properties, and only few reports have shown electrochemical results by clear redox peaks in the cyclic voltammetry, and discharge capacities of $\sim 125 \mathrm{mAh} \cdot \mathrm{g}^{-1}$ with clear plateau regions in the charge-discharge curves.[63,65,66]

Detailed insight into the relation between the specific crystal orientation toward the adjacent electrolyte and its electrochemical behavior has been lacking, which has hampered the successful development of high-quality $\mathrm{LiMn}_{2} \mathrm{O}_{4}$ cathode with high cyclability. A detailed study by Hirayama et al. concluded from surface X-ray diffraction measurements that a SEI was present on both (111) and (110) surfaces, although the (110) surface was less stable and indicated a higher Mn dissolution.[62] So far the electrochemical performance was only reported for $\mathrm{LiMn}_{2} \mathrm{O}_{4}$ thin films grown on (111)oriented $\mathrm{SrTiO}_{3}$ substrates, $[63,66]$ where an additional $\mathrm{Li}_{3} \mathrm{PO}_{4}$ coating was added to prevent a phase transition of the surface region and to suppress Mn dissolution and desorption of oxygen from the surface.

By structural engineering of stable, epitaxial $\mathrm{LiMn}_{2} \mathrm{O}_{4}$ thin films the electrochemical properties can be controlled and enhanced as compared to polycrystalline samples. In this section, the control over the crystallographic orientation of LMO thin films is demonstrated through PLD growth. By changing the crystal orientation of the underlying single crystalline substrate ((100), (110) and (111)), the specific orientation of the 
$\mathrm{LiMn}_{2} \mathrm{O}_{4}$ thin film can be controlled and, therefore, the cathode surface toward the adjacent electrolyte, allowing the analysis of the relation between structural and electrochemical properties.

\subsubsection{LMO thin film growth and characterization}

The structural quality of the LMO films was investigated by XRD analysis, as shown in Figure 2-8. The three types of LMO films grown on Nb:STO substrates with different orientations exhibit coherent growth in which the out-of-plane crystal orientation of the films is aligned with the orientation of the substrate. A $50 \mathrm{~nm} \mathrm{SrRuO}_{3}$ (SRO) layer was deposited as an intermediate layer to enhance the electrical transport between the $\mathrm{LiMn}_{2} \mathrm{O}_{4}$ cathode and the conducting $\mathrm{Nb}: \mathrm{STO}$ substrate.[63]

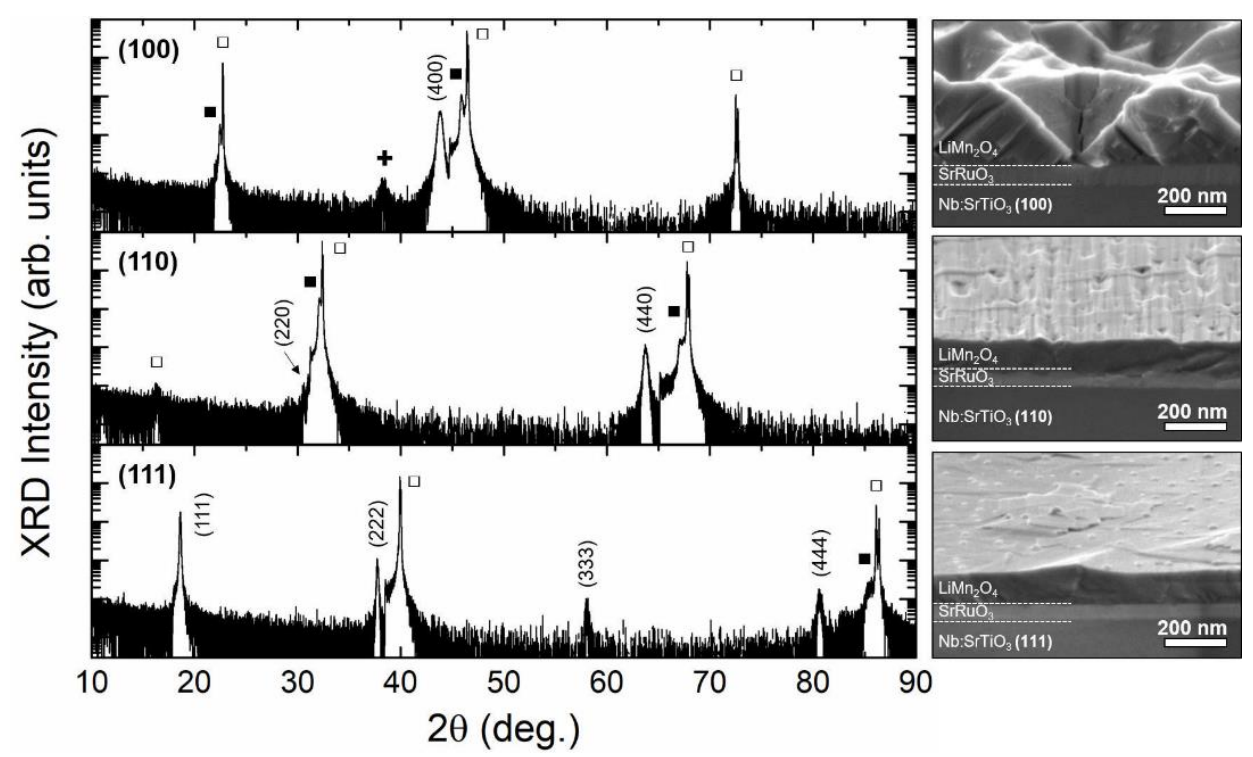

Figure 2-8. (left) Out-of-plane XRD measurements of $110 \mathrm{~nm} \mathrm{LiMn}_{2} \mathrm{O}_{4}$ epitaxial thin films on $50 \mathrm{~nm} \mathrm{SrRuO}_{3}$-buffered $\mathrm{Nb}: \mathrm{SrTiO}_{3}$ substrates with different crystal orientations: (100), (110), and (111). Nb:SrTiO 3 substrate peaks are indicated by $\square, \mathrm{LiMn}_{2} \mathrm{O}_{4}$ peaks by $\downarrow$, and $\mathrm{SrRuO}_{3}$ are indicated by $\mathbf{m}$, whereas minor contributions of $\mathrm{Mn}_{2} \mathrm{O}_{3}$ phase are given

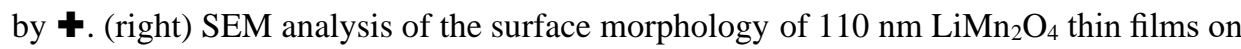
$\mathrm{SrRuO}_{3}$-buffered $\mathrm{Nb}: \mathrm{SrTiO}_{3}$ substrates with crystal orientations (100), (110) and (111). SEM images are taken after extensive electrochemical cycling and subsequent cleaning of the surfaces. 
The $\operatorname{LMO}(111)$ and $\mathrm{LMO}(110)$ films show the presence of highly crystalline epitaxial layers, with a cubic lattice parameter of $\sim 8.25 \AA$, without any impurity phase, in good agreement with previous studies of LMO growth on $\operatorname{STO}(111)$ and STO(110) substrates.[59,63] This suggests that the PLD process parameters (e.g. temperature, pressure, laser energy density, target composition) were optimized successfully to correct for any loss of volatile lithium during ablation, nucleation or growth. Interestingly, the LMO films with (100)-orientation show minor contributions of a secondary phase, although all three LMO films were grown during the same deposition procedure. The extra peaks suggest the presence of a small amount of $\mathrm{Mn}_{2} \mathrm{O}_{3}$, [67] which will have negligible effect on the electrochemical performance of the (100)-oriented $\mathrm{LiMn}_{2} \mathrm{O}_{4}$ thin films, as the anodic reduction and cathodic oxidation reactions in $\mathrm{Mn}_{2} \mathrm{O}_{3}$ take place below $\sim 1.3$ V. $[67,68]$ This is far below the potential window in the measurements of $3.6-4.5$ $\mathrm{V}$ performed. The coexistence of this lithium deficient phase could be due to the enhanced lithium volatility at the (100) surface of $\mathrm{LiMn}_{2} \mathrm{O}_{4}$.[49]

The alignment of the out-of-plane crystal orientation for all types of LMO films, suggests an epitaxial relation between the crystal structures of the deposited LMO films and the underlying $\mathrm{Nb}: \mathrm{STO}$ substrates, although large differences exist between spinel LMO ( $a=8.25 \AA$ ) and perovskite STO $(a=3.91 \AA)$. The surface of the LMO(100) film exhibits square-like structures with significant height differences (Rms $\sim 45 \mathrm{~nm}$ ), which is in good agreement with previously observed octahedron spinel structures.[27,58] Such pyramidal spinel structures consist of $\langle 111\rangle$ crystal facets on all four sides with an occasional presence of a truncated top of the pyramid exhibiting a (100) crystal facet. The LMO(110) film forms a layer with rooftop like structures and a lower surface roughness (Rms $\sim 5 \mathrm{~nm}$ ), caused by the anisotropic nature of the (110)-plane which favors diffusion of atoms along the [110] direction as compared to the [001] direction.[28] This results in elongated $\langle 111\rangle$ crystal facets exposed on the surface, which are all aligned in the same direction. Finally, the $\mathrm{LMO}(111)$ film forms a layer with triangle-like structures exhibiting a very low surface roughness $(\mathrm{Rms} \sim 1.5 \mathrm{~nm})$. The triangular shape corresponds to the (111) plane in a cubic structure, for which two different types of in-plane triangle orientations can be observed. Therefore, all three types of LMO films with different outof-plane orientations ((100), (110) and (111)) exhibit surfaces exposing predominantly $\langle 111\rangle$ crystal facets, which confirms that this is the lowest energy state surface of the spinel crystal structure.[58]

To study the dependence of the lithium transport on the specific crystal orientation of the LMO films, samples were cycled galvanostatically between 3.6 and $4.5 \mathrm{~V}$ with currents of $1,2,5,10,20$, and $50 \mu \mathrm{A}$, corresponding to $\mathrm{C}$ rates of approximately $0.7,1.3$, $3.3,6.6,13$, and $33 \mathrm{C}$, respectively, against lithium metal with a liquid electrolyte. Figure 2-9 shows charge-discharge curves for the LMO films with different orientations ((100), (110), and (111)) for various currents, resulting in (dis)charge rates in the range $0.7-33 \mathrm{C}$. 

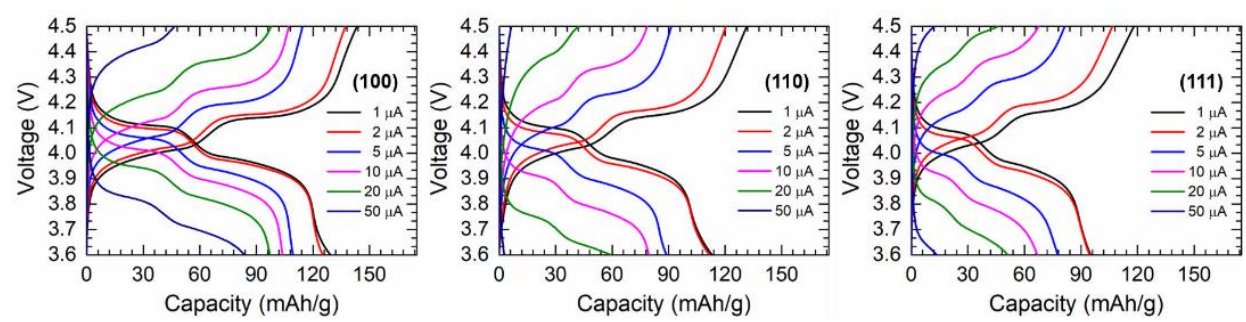

Figure 2-9. Charge-discharge analysis of $110 \mathrm{~nm} \mathrm{LiMn}_{2} \mathrm{O}_{4}$ films with different crystal orientations ((100), (110) and (111)) for various currents $(1,2,5,10,20$, and $50 \mu \mathrm{A})$. A potentiostatic period of $5 \mathrm{~min}$ is used to ensure complete charge or discharge before the next step.

The characteristic voltage plateaus for these epitaxial LMO thin films are in good agreement with bulk LMO charge-discharge profiles.[8] The total discharge capacity for the slowest rate of $0.7 \mathrm{C}$ was the highest for the (100)-oriented LMO film $\left(\sim 129 \mathrm{mAh} \cdot \mathrm{g}^{-1}\right)$, whereas the (110)- and (111)-oriented LMO films exhibit lower discharge capacities of respectively $\sim 113$ and $\sim 95 \mathrm{mAh} \cdot \mathrm{g}^{-1}$. The large surface area of the (100)-oriented LMO film, caused by pyramidal surface morphology, is considered to cause enhanced lithium kinetics as compared to the other crystal orientations. The crystal facets on all films are predominantly $\langle 111\rangle$, which eliminates any possible effect from local variations in crystal facets. The enhanced lithium kinetics for the (100)-oriented LMO films is also demonstrated by the large capacities still achievable during (dis)charging when using higher rates (33C), as compared to polycrystalline studies.[51] The high currents used stress the material and make the variations in lithium intercalation for the different crystal orientations more pronounced. For currents of $20 \mu \mathrm{A}(\sim 13 \mathrm{C})$, the discharge capacities for the (110)- and (111)-oriented films drop to $\sim 50 \mathrm{mAh} \cdot \mathrm{g}^{-1}$, whereas the (100)-oriented film still exhibits double the capacity $\left(\sim 100 \mathrm{mAh} \cdot \mathrm{g}^{-1}\right)$. The initial drop in discharge capacity after the first charge-discharge cycle may be attributed to anionic reaction occurring at upper voltage cutoff combined with irreversible dissolution of surface lithium and manganese.[56] Furthermore, it is interesting to note that initially at low currents, all films show a slightly higher charge capacity compared to the discharge. Although the exact origin is still unclear, the difference in charge-discharge capacities are within acceptable Coulombic efficiency limits (> 95\%).

\subsection{Investigating the ionic conductivity of $(\mathrm{Li}, \mathrm{La}) \mathrm{TiO}_{3}$ thin films}

Although many non-oxide solid electrolytes with high lithium-ion conductivities, including $\mathrm{Li}_{3} \mathrm{~N}\left(\sigma_{300 \mathrm{~K}}=1.2 \times 10^{-3} \mathrm{~S} \cdot \mathrm{cm}^{-1}\right)$, [69] thio-LISICON $\left(\mathrm{Li}_{3.25} \mathrm{Ge}_{0.25} \mathrm{P}_{0.75} \mathrm{~S}_{4}, \sigma_{300 \mathrm{~K}}=\right.$ $\left.2.2 \times 10^{-3} \mathrm{~S} \cdot \mathrm{cm}^{-1}\right),[70] \mathrm{Li}_{7} \mathrm{P}_{3} \mathrm{~S}_{11}\left(\sigma_{300 \mathrm{~K}}=3.2 \times 10^{-3} \mathrm{~S} \cdot \mathrm{cm}^{-1}\right),[71]$ and $\mathrm{Li}_{10} \mathrm{GeP}_{2} \mathrm{~S}_{12}\left(\sigma_{300 \mathrm{~K}}=\right.$ $\left.1.2 \times 10^{-2} \mathrm{~S} \cdot \mathrm{cm}^{-1}\right),[12]$ have been proposed, they are unsuitable for practical applications 
because their constituents easily decompose and oxidize when exposed to air. Electrolytes composed of lithium-ion conducting oxides have potential advantages due to chemical and thermal stability. Since the discovery of its high lithium-ionic conductivity $\left(\sigma_{300 \mathrm{~K}}\right.$ $\sim 10^{-3} \mathrm{~S} \cdot \mathrm{cm}^{-1}$ ) by Inaguma et al.,[72] lithium lanthanum titanate $\mathrm{Li}_{3 \mathrm{x}} \mathrm{La}_{2 / 3-\mathrm{x}} \square_{1 / 3-2 \mathrm{x}} \mathrm{TiO}_{3}$ (LLTO) has attracted much attention as promising candidate for lithium-ion conducting solid electrolytes.[73] However, the current performance of LLTO as solid electrolyte remains insufficient due to its high grain boundary interfacial resistance, which could be overcome via interface engineering, provided by thin film technology.

LLTO has a lattice parameter $(a=3.86-3.88 \AA[74,75])$ which matches with the $\mathrm{Nb}: \mathrm{SrTiO}_{3}$ substrate $(a=3.91 \AA)$ and half of the unit cell of the LTO and LMO spinel phases $(a=4.12-4.18 \AA$ ), having a high probability to grow epitaxially on the $\mathrm{Nb}: \mathrm{SrTiO}_{3}$ substrates and the chosen electrodes. The crystal structure of the perovskite LLTO is shown in Figure 2-10a. The LLTO unit cell is commonly represented by an orthorhombic structure of two stacked primitive perovskite unit cells due to A-site defects $(\square)$ in the crystal structure.[76,77] However, when the lithium ratio (x) in LLTO is sufficiently high $(x>0.08)$, the crystal structure is regarded as cubic.[78,79] The stoichiometry of LLTO has a dramatic effect on its ionic conductivity and lattice parameter,[80] e.g. for $x=0.11\left(\mathrm{Li}_{0.33} \mathrm{La}_{0.56} \mathrm{TiO}_{3}\right)$ the highest ionic conductivity is achieved, due to lithium vacancy controlled diffusion through the LLTO-system.[72,81] Ionic diffusivity within the LLTO structure occurs preferably in-plane, due the unequal distribution of $\mathrm{La}^{3+}$ ions, [82] causing an anisotropy in diffusion pathways which should be considered on the assembly of a full solid-state battery, as the electrodes set the electric field to a specific direction.

To study the influence of crystal orientation on the ionic conductivity of LLTO, thin films were grown using the parameters presented in Table $2-1\left(900{ }^{\circ} \mathrm{C}, 20 \mathrm{~Hz}\right.$ and 0.2 mbar), with stoichiometric target with La:Li (1:1) on (100) and (111) orientated Nb:STO crystalline substrates. It's expected for the (111) direction to show higher out-of-plane ionic conductivity as both a- and b-axis contribute for the conduction, whereas the (100) direction show conduction pathways parallel to the surface. The structural quality of the LLTO films was investigated by XRD analysis, as shown in Figure 2-10b. The two types of LTO films grown on Nb-doped STO substrates with different orientations exhibit coherent growth in which the out-of-plane crystal orientation of the films is aligned with the orientation of the substrate. The LLTO(111) film shows the presence of a highly crystalline epitaxial layer, with a lattice parameter of $\sim 3.87 \AA$, with the presence of $\mathrm{Li}$ deficient impurity phases $\left(\mathrm{TiO}_{2}\right.$-Anatase and $\left.\mathrm{La}_{2} \mathrm{Ti}_{2} \mathrm{O}_{7}\right)$. Anatase is commonly present on LLTO thin films $[75,83]$ and $\mathrm{La}_{2} \mathrm{Ti}_{2} \mathrm{O}_{7}$ has minor contributions to the structure, due its low XRD peak intensity, suggesting that the PLD process parameters (e.g. temperature, pressure, laser energy density, target composition) were optimized for the growth of LLTO. Interestingly, the LLTO film with (100) orientation shows higher contributions of a Li-deficient secondary phase $\left(\mathrm{TiO}_{2}\right.$-Anatase), although both films were grown with the 
same PLD parameters. As observed for the materials studied previously in this chapter (LTO and LMO), the coexistence of a lithium deficient phase could be attributed to the enhanced lithium volatility at the (100) surface.

(a)

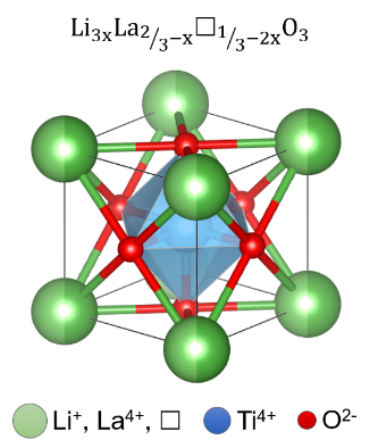

(b)

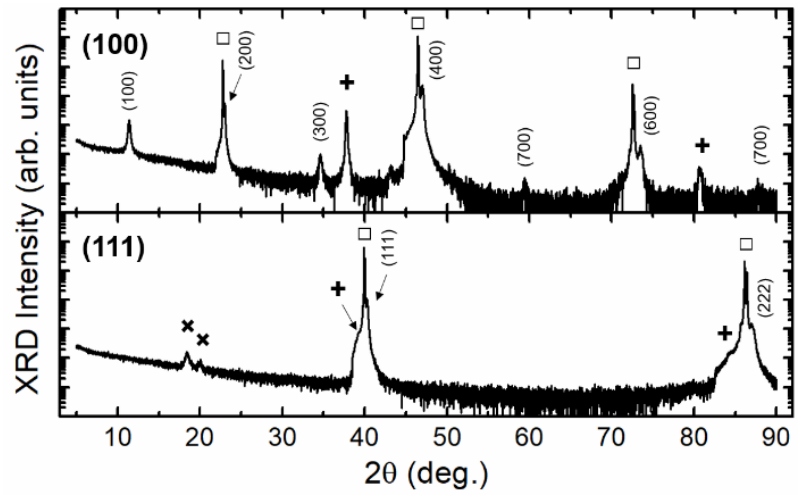

Figure 2-10. (a) Schematic of the perovskite cubic unit cell $(x=0.167)$ LLTO. (b) Outof-plane XRD measurements of $100 \mathrm{~nm}$ LLTO epitaxial thin films on $\mathrm{Nb}: \mathrm{SrTiO}_{3}$ substrates with different crystal orientations: (100) and (111). LLTO peaks are indicated by the (hkl) indices, $\mathrm{Nb}: \mathrm{SrTiO}_{3}$ substrate peaks are indicated by $\square$, Li-deficient phase anatase $\left(\mathrm{TiO}_{2}\right)$ by $\mathbf{+}$, whereas minor contributions of $\mathrm{La}_{2} \mathrm{Ti}_{2} \mathrm{O}_{7}$ phase are given by $\mathbf{x}$.

The ionic conductivity of LLTO thin films grown with different orientations were compared using Electrochemical Impedance Spectroscopy (EIS). EIS allows the modeling of electrochemical behavior of systems in detail by separating the behaviors of the different components in the time domain, allowing to decouple the ionic and electronic processes taking place within the material. By measuring the current response for small $\mathrm{AC}$ voltage $\left(10 \mathrm{mV}_{\mathrm{pp}}\right)$ perturbations, at different frequencies $(1 \mathrm{MHz}-1 \mathrm{~Hz})$ while the system is in thermodynamic equilibrium, the impedance as a function of frequency can be analyzed. For the analysis it is assumed that the system does not change over time. The quality of the measurements is checked by comparing the accuracy (pseudo- $\chi^{2}$ ) of the fit and the Kramers-Kronig relation (KK), where the Equivalent Circuits (EC) were adjusted to guarantee a minimum difference between them, while representing a realistic physical system. The measurements were performed at $22{ }^{\circ} \mathrm{C}$ using a BioLogic VMP-300 and an EC-ref cell by EL-CELL. For the temperature dependent study, Ivium vertex was used. All the films were covered by a gold sputtered layer for better electronic contact and dried in the glovebox for 10 minutes at $130{ }^{\circ} \mathrm{C}$ before the measurements. 


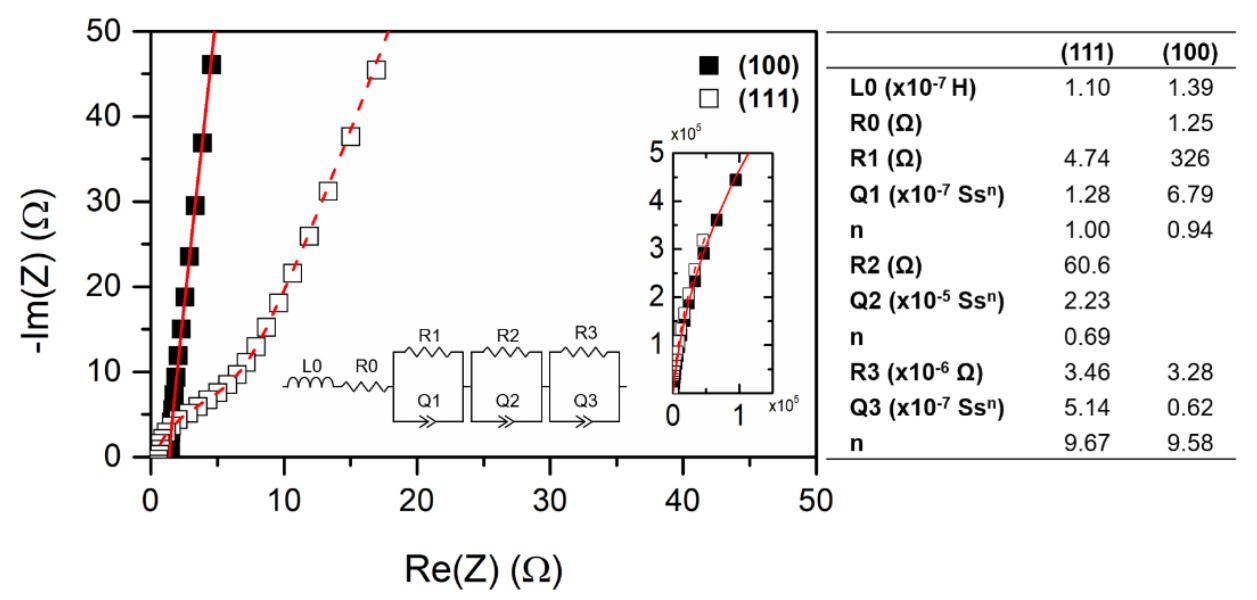

Figure 2-11. Complex plane plot for the LLTO grown on (100) and (111) oriented $\mathrm{Nb}$ :STO with their respective fittings. The inset show the equivalent circuit used for the fit, together with the data over the full range, and the values for the fitting can be found on table on the right.

The EIS measurements, plotted in complex plane plots $(-\operatorname{Im}(Z)$ vs. $\operatorname{Re}(Z))$, for the LLTO films grown on the (100) and (111) orientations are shown in Figure 2-11, with their respective fits and equivalent circuit. The transition in behavior observed for the (111) curve occurs at $171.6 \mathrm{kHz}$. Although parts of the EC are used to represent the wires and electrodes [R0L0], or the interface between the electrodes and the film (R3Q3), the discussion will be focused on the values of (R1Q1), which correspond to the sample properties and present capacitances on the same order of magnitude. The (R2Q2) component is present only for the (111) orientation, which could be dependent on the reactivity of the $\langle 111\rangle$ facet, and the exposure time of the sample with air before the measurement. As reported by Bohnke et al., [84] LLTO can interact with $\mathrm{H}_{2} \mathrm{O}$ and $\mathrm{CO}_{2}$ present in air to form $\mathrm{Li}_{0.30-y} \mathrm{H}_{\mathrm{y}} \mathrm{La}_{0.57} \mathrm{TiO}_{3}$ and $\mathrm{Li}_{2} \mathrm{CO}_{3}$, respectively. From the fitted values, the ionic resistance of the electrolyte on the (111) orientation $(\sim 4.7 \Omega)$ is two orders of magnitude lower than for the (100) $(\sim 346 \Omega)$. The tetragonal structure (Figure 2-10) obtained for the LLTO could result in a low ionic conductivity as the Li pathways are perpendicular to the c-axis and the direction of the applied electric field. The values for interface resistance and wire inductance are in the expected range.

The presence of impurity phases $\left(\mathrm{La}_{2} \mathrm{Ti}_{2} \mathrm{O}_{7}\right.$ and $\left.\mathrm{TiO}_{2}\right)$ could have influence over the measurements, lowering the ionic conductivity, as anatase's ionic conductivity is orders of magnitude lower than LLTO $\left(\sigma_{\text {anatase }}=1.8 \times 10^{-13} \mathrm{~S} \cdot \mathrm{cm}^{-1}[85]\right)$. But since both films have the presence of the Li-deficient phases, it was considered that the impurities wouldn't affect the comparison in conductivity. Another explanation for the difference in ionic conductivity, as demonstrated by Hasegawa et al.,[82] is the difference in strain between the (100) and (111) orientation. The activation energy of Li ionic conduction is 
increased with a compressive strain, resulting in contracted ionic conduction paths, which has a similar tendency to pressure and substitution effect in bulk study.
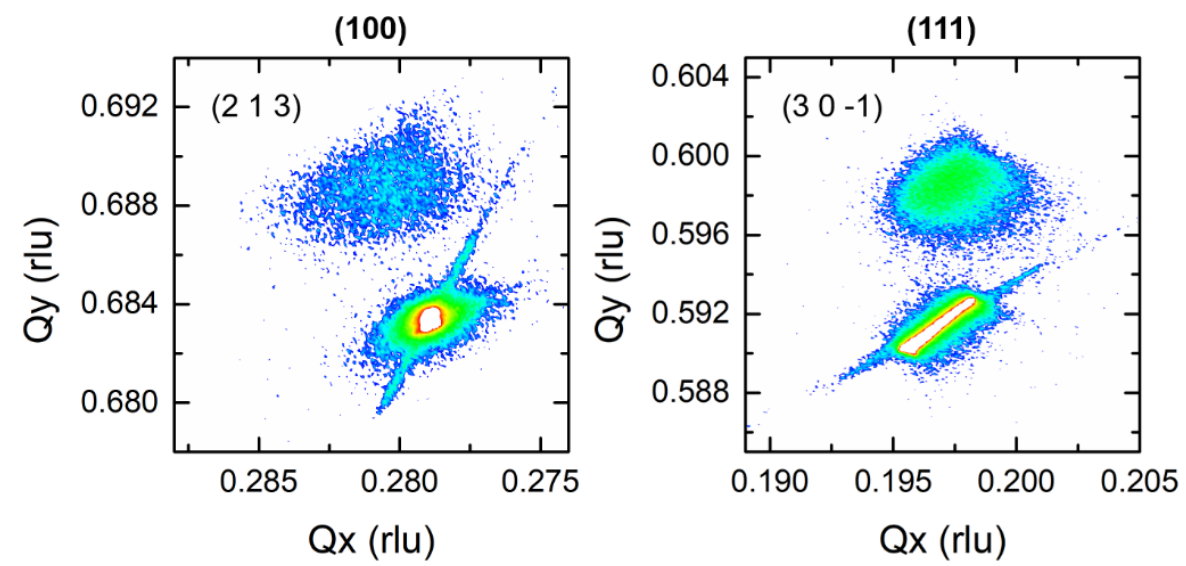

Figure 2-12. Reciprocal Space Maps for $\sim 110 \mathrm{~nm}$ LLTO thin film grown with the (100) orientation, measured along the (3 0 -1) plane, and the (111) orientation, measured along the $\left(\begin{array}{lll}2 & 1 & 3\end{array}\right)$ plane.

Figure 2-12 show Reciprocal Space Maps (RSM) for the (111) and (100) grown LLTO films, which adds in-plane information. On the (111) orientation, a partially relaxed film is grown which, according to Hasegawa et al.,[82] would favor the ionic conductivity. Whereas in the (100) direction a strained film is grown, decreasing the bottlenecks for diffusion, increasing the ionic resistivity.

\subsubsection{Temperature dependence analysis for LLTO ionic conductivity}

To calculate the activation energy for ionic conduction of $\sim 450 \mathrm{~nm}$ of the film grown at $900{ }^{\circ} \mathrm{C}$, a temperature dependent measurement was performed. Since the spinel electrodes mentioned in Section 2.3 and 2.4 grow smoother on the (111) direction, and the goal is to combine all three components in a full battery stack, LLTO(111) was chosen for this study. The ionic conductivity was calculating using Equation 2.1:

$$
\sigma=\frac{d}{R A}
$$

Where $d$ is the thickness of the film, $A$ is the surface area and $R$ is the ionic resistance obtained by EIS. In ionically conducting crystals, the hopping motion of charged defects 
(ions and vacancies within the structure) is what causes ionic conduction. [86] The lithium diffusion in crystals can be described by the Nernst-Einstein equation:[87]

$$
D=\frac{\mu k_{B} T}{q}
$$

Where $D$ is the diffusion coefficient, $\mu$ is the electrical mobility, $k_{B}$ is the Boltzmann constant, $T$ is the temperature in Kelvin and $q$ is the charge of the diffusing ion. The diffusion of $\mathrm{Li}^{+}$ions in solid state electrolytes can be modeled by the Arrhenius-type relation:

$$
D=D_{0} e^{\frac{-E_{A}}{k_{B} T}}
$$

Where $D_{0}$ is the diffusion constant and $\mathrm{E}_{\mathrm{A}}$ is the activation energy. The relation between conductivity and mobility is given by Equation 2.4:

$$
\sigma=n q \mu
$$

Where $\sigma$ is the ionic conductivity and $n$ is the concentration of moving ions. Combining Equations 2.2 and 2.4, Equation 2.3 can be rewritten as:

$$
\sigma=\frac{\sigma_{0}}{T} e^{\frac{-E_{A}}{k_{B} T}}
$$

Equation 2.5 shows the dependence of conductivity with temperature. Figure 2-13a shows EIS measurements for the LLTO film for different temperatures, with their respective fits and EC. At room temperature, the film show an ionic conductivity of 22.4 $\mu \mathrm{S} \cdot \mathrm{cm}^{-1}$, comparable with other studies on crystalline LLTO.[74,82] With the values of ionic resistivity obtained, using Equation 2.1, the Arrhenius plot in Figure 2-13b was drawn, with a slope of $-4320 \mathrm{~K}$. From Equation 2.5, an activation energy of $0.35 \pm 0.02$ $\mathrm{eV}$ is obtained, also comparable with other studies of LLTO(111) on STO.[74,82]

Although the value for $\mathrm{E}_{\mathrm{A}}$ obtained is similar with what others have reported, it is still higher than the theoretical values $(0.24 \mathrm{eV}$ [88]). On a study of the influence of domain boundaries on Li migration in LLTO, Moriwake et al. calculated the activation energy for diffusion for Li-ions diffusing through different barriers, summarized in Table 22.[89] Thus, a possible explanation for the difference between the theoretical ionic conductivity of $10^{-3} \mathrm{~S} \cdot \mathrm{cm}^{-1}$ and the ionic conductivity observed in thin films could be the formation of domain boundaries, resulting in La-rich layers, which would increase the total activation energy. 

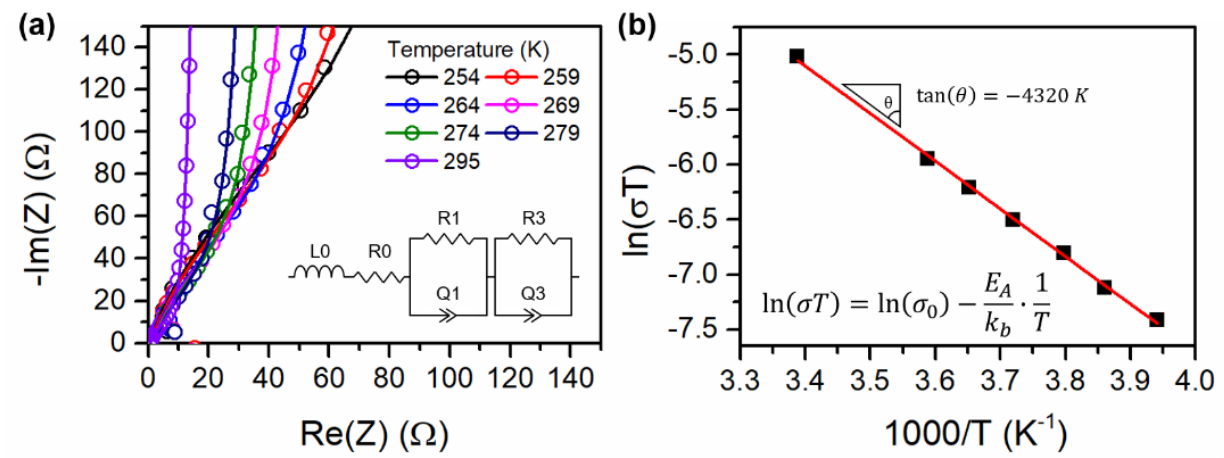

Figure 2-13. (a) Complex plane plot for the LLTO grown on (111) oriented Nb:STO with for different temperatures. The inset show the equivalent circuit used for the fit, together with the data over the full range.

Table 2-2. Activation energy, conductance and resistance for different conduction mechanisms in LLTO, extracted from [89]. The resistance is calculated for a $90 \mathrm{~nm}$ thick film, with surface area of $16 \mathrm{~mm}^{2}$.

\begin{tabular}{lccc}
\hline Conduction Mechanism & $\mathbf{E}_{\mathbf{A}}(\mathbf{e V})$ & $\boldsymbol{\sigma}\left(\mathbf{S} \cdot \mathbf{c m}^{-\mathbf{1}}\right)$ & $\mathbf{R}(\mathbf{\Omega})$ \\
\hline Through La-rich layer without La vacancy & 3.58 & $4.22 \times 10^{-60}$ & $1.33 \times 10^{55}$ \\
Through La-rich layer with La vacancy & 0.58 & $7.50 \times 10^{-9}$ & $7.50 \times 10^{3}$ \\
Through Li-rich layer with Li vacancy & 0.26 & $2.20 \times 10^{-3}$ & $2.56 \times 10^{-2}$ \\
\hline
\end{tabular}

The total ionic conduction in the film can be described according to Equation 2.6 if the migration of $\mathrm{Li}^{+}$is only through $\mathrm{La}$ or $\mathrm{Li}$ vacancies:

$$
R_{\text {total }}=R_{L i} \frac{x}{d}+R_{L a} \frac{y}{d}
$$

Where $R_{\text {total }}$ is the total ionic resistivity of the thin film, $R_{L i}$ is the ionic resistivity of a Liion moving through $\mathrm{Li}$ vacancy, $R_{L a}$ is the ionic resistivity of a $\mathrm{Li}$-ion moving through $\mathrm{La}$ vacancy, $x$ is the thickness of the layer with a resistance of $R_{L i}, y$ is the thickness of layer with a resistance of $R_{L a}$, and $d$ is the total thickness of the film. The ratios $x / d$ and $y / d$ can be obtained, considering that $x+y=d$, resulting in Equations 2.7 and 2.8:

$$
\begin{aligned}
& \frac{x}{d}=\frac{R_{t o t a l}-R_{L a}}{R_{L i}-R_{L a}} \\
& \frac{y}{d}=\frac{R_{t o t a l}-R_{L i}}{R_{L a}-R_{L i}}
\end{aligned}
$$


For the film with an ionic conductivity of $22.4 \mu \mathrm{S} \cdot \mathrm{cm}^{-1}$, the calculated $x / d$ and ${ }^{y} / d$ ratios are 0.9979 and 0.0021 , respectively. This simple model demonstrates that a minor contribution of La-rich layers can significantly increase the overall activation energy for ionic diffusion, confirming the importance of the control over the film growth. Transmission Electron Microscopy (TEM) could be used to confirm if the model holds, by measuring the amount of domain boundaries within the film. As mentioned before, the (111) film is partially strained and, although it is known that in-plane strain can influence the ionic conductivity,[82] this factor was disregarded in this calculation.

\subsection{Planar 2D all-solid-state battery}

From the knowledge obtained from the previous sections, it is now possible to synthesize the three key elements of the battery, namely the anode $\left(\mathrm{Li}_{4} \mathrm{Ti}_{5} \mathrm{O}_{12}-\mathrm{LTO}\right)$, solid electrolyte $\left(\mathrm{Li}_{3 x} \mathrm{La}_{2 / 3-x} \mathrm{TiO}_{3}-\mathrm{LLTO}\right)$, and cathode $\left(\mathrm{LiMn}_{2} \mathrm{O}_{4}-\mathrm{LMO}\right)$. As already mentioned, the use of a solid electrolyte improves the safety and energy density of a battery, with the disadvantage of low ionic diffusivity. However, a reduction in thickness, as the electrolyte can be just thick enough to avoid a short circuit between the electrodes, could be enough to compensate for the reduction in ionic diffusion coefficient. Thinner solid-state electrolyte and the choice of high potential cathodes, would solve the main issues regarding solid-state batteries.[90]

To synthesize a full stack of the materials, crucial limitations must be considered. The deposition temperature of $\mathrm{LMO}$ is limited to around $600{ }^{\circ} \mathrm{C}$, as higher temperatures result in a structural change which is not electrochemically active. As both the anode LTO and the electrolyte LLTO require higher deposition temperatures of respectively $700{ }^{\circ} \mathrm{C}$ and $900{ }^{\circ} \mathrm{C}$ they must be deposited before LMO. With voltages of $1.5 \mathrm{~V}$ and $4 \mathrm{~V}$ versus $\mathrm{Li} / \mathrm{Li}^{+}$ for the anode and cathode materials, respectively, a potential difference of $2.5 \mathrm{~V}$ is expected. The parameters used for the growth of the full 2D solid-state battery stack are the same as the ones used for the materials individually, presented in Table 2-3, with thickness of 170, 120 and $140 \mathrm{~nm}$ for the LTO, LLTO and LMO layers, respectively. For the thickness and Li density of the LMO grown on the (111)-oriented substrate, the LTO layer thickness was adjusted [36] to intercalate the same amount of lithium ions that is extracted from LMO layer upon charge. The three materials were deposited in the same PLD chamber.

Y. Zhu et al. [91] showed that the LLTO can form an unstable interphase on the interface with Li-metal anode or the oxide cathodes, which accelerates the decomposition, as it is thermodynamically favorable. To avoid the decomposition of the electrolyte, for the anode, LTO is chosen as many electrolytes decompose at low voltages versus the reactive $\mathrm{Li} / \mathrm{Li}^{+}$.[92,93] Additionally, other studies have shown LLTO to be stable in combination with LMO, as cathode.[74] 


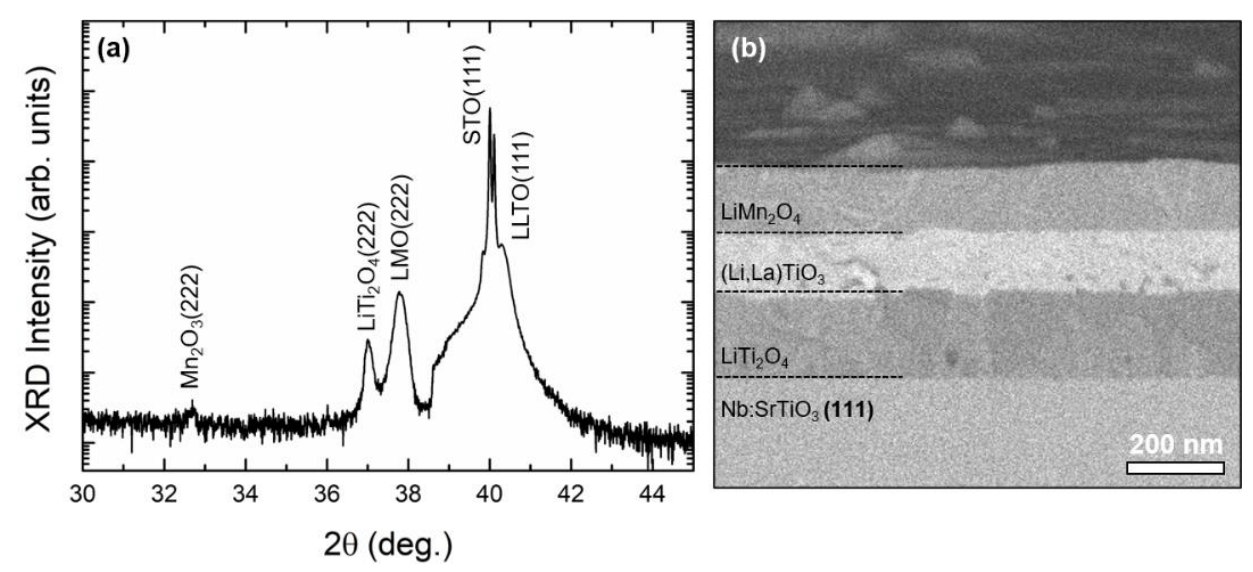

Figure 2-14. (a) XRD pattern for the full battery stack on (111)-oriented Nb:STO substrate. Peaks for LMO, LLTO and a Li-deficient LTO phase are highlighted, together with a small presence of (111) $\mathrm{Mn}_{2} \mathrm{O}_{3}$. (b) ESB image showing composition contrast.

The (111) orientation is chosen because both LTO and LMO layers grown on (111)oriented substrates show lower surface roughness than in other orientations, as expected for a spinel material grown on a (111)-oriented perovskite substrate [94]. The flat surface would allow the consecutive layers to also grow smoothly. The crystallinity of the multilayer was investigated by XRD (Figure 2-14a). The peaks of the STO substrate can be observed, together with peaks for LMO, LTO and LLTO. All the layers grow on the same orientation of the substrate. Although the $\mathrm{Li}_{4} \mathrm{Ti}_{5} \mathrm{O}_{12}$ phase was expected for the LTO thin film grown, a lithium deficient phase $\left(\mathrm{LiTi}_{2} \mathrm{O}_{4}\right)$ is present. The higher temperature of the consecutive layer $\left(900{ }^{\circ} \mathrm{C}\right.$ for the LLTO) could explain the loss of lithium on the anode. The all-solid-state battery stack formed is shown in the SEM images, in Figure 2-14b. Cross-sectional Energy selective Backscattered (ESB) SEM image is shown in Figure 2-14c, with the contrast given by difference in atomic mass of the materials, with heavier elements appearing brighter.

To study the electrochemical properties of the layered 2D battery, the whole stack was analyzed with EIS. The results and its equivalent circuit fit are presented in Figure 2-15. From this circuit, obtained values for wires, electrode and electrolyte are comparable to previous results, although the value for the charge transfer resistance (R3) is higher. The impedance results show no electrical short, as shorts are characterized by a single point in the Nyquist plot. Cyclic voltammetry measurements were also performed on these films, however no characteristic intercalation peaks were observed, which could be a result of the higher charge transfer resistance (R3), the nonoptimal LLTO electrolyte, and/or the lithium deficient LTO phase present. Future work is recommended on the optimization of the combined materials. 


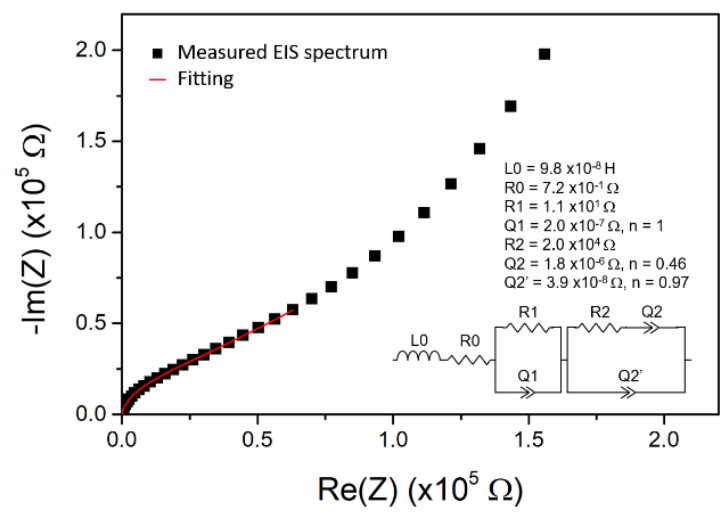

Figure 2-15. Electrochemical Impedance measurement of the full 2D solid-state battery stack, with the equivalent circuit and the fitted data. The fitting was done in frequencies above $12.8 \mathrm{~Hz}$.

\subsection{Conclusion}

Improved control over the electrochemical properties of $\mathrm{Li}_{4} \mathrm{Ti}_{5} \mathrm{O}_{12}, \mathrm{LiMn}_{2} \mathrm{O}_{4}$ and $\mathrm{Li}_{0.5} \mathrm{La}_{0.5} \mathrm{TiO}_{3}$ thin films is enabled by structural engineering, which is unique for epitaxial thin films and cannot be obtained in single-crystal or polycrystalline samples. Dramatic differences in surface morphology with pyramidal, rooftop, or flat nanostructures were achieved by controlling of the specific crystal orientation, respectively (100), (110), and (111), for the spinel LTO and LMO thin films. The exposed surface is predominantly $\langle 111\rangle$ for all three types of morphology, showed experimentally, and confirmed by the phase field model, to enhance the lithium storage of spinel thin film electrodes due to significant surface contributions. All three crystal orientations showed high electrochemical performance with good cyclability without any significant capacity fading, as well as high discharge capacities. The (100)-oriented LMO films exhibited the highest capacities and (dis)charging rates up to 33C, demonstrating enhanced cycle life without excessive capacity fading as compared to previous polycrystalline studies; while LTO thin films showed capacity of $280-310 \mathrm{mAh} \cdot \mathrm{g}^{-1}$ exceeding its theoretical capacity of $175 \mathrm{mAh} \cdot \mathrm{g}^{-1}$. The layer thickness dependence study demonstrated that these high capacities are caused by large surface contributions in lithium storage, explained by the contribution of the SEI layer. The (100)-oriented films exhibit an enhanced surface area for its pyramidal surface morphology, showing the highest surface capacities. Structural engineering for the LLTO thin films, together with EIS, showed the orientation dependence on the ionic diffusion for the solid electrolyte, as the Li-diffusion pathways are oriented in-plane, considering the tetragonal unit cell. Activation energies for hopping were calculated by a temperature dependence measurement, highlighting that a minor 
contribution of La-rich layers on the domain boundaries, could hinder ionic diffusion in thin films. Finally, with the knowledge acquired for the three components of the battery, a full solid-state battery stack was grown. XRD measurements show the correct phases of LMO and LLTO, with a Li-deficient phase for LTO, which could be the result of the higher temperatures required for the LLTO growth. EIS measurements do not show a short circuit between the layers, although cycling the full stack was not possible. Optimization of these batteries should focus on finding the optimum growth conditions for the combined materials to improve their interface or developing an intimate combination with a solid electrolyte in a vertically aligned 3D nanocomposite, as it will be focused on the next chapters.

\section{Bibliography}

[1] Winter, M., Brodd, R. J., What are batteries, fuel cells, and supercapacitors? Chem. Rev. 2004, 104, 4245-4269.

[2] Cheng, F., Liang, J., Tao, Z., Chen, J., Functional materials for rechargeable batteries. Adv. Mater. 2011, 23, 1695-1715.

[3] Oudenhoven, J. F. M., Vullers, R. J. M., Schaijk, R., A review of the present situation and future developments of micro-batteries for wireless autonomous. Int. J. energy Res. 2012, 36, 135-147.

[4] Zhao, B., Ran, R., Liu, M., Shao, Z., A comprehensive review of $\mathrm{Li}_{4} \mathrm{Ti}_{5} \mathrm{O}_{12}$-based electrodes for lithium-ion batteries: The latest advancements and future perspectives. Mater. Sci. Eng. R 2015, 98, 1-71.

[5] Yuan, T., Tan, Z., Ma, C., Yang, J., Ma, Z. F., Zheng, S., Challenges of Spinel Li4Tis $\mathrm{O}_{12}$ for Lithium-Ion Battery Industrial Applications. Adv. Energy Mater. 2017, 7, 1601625.

[6] Xu, G., Han, P., Dong, S., Liu, H., Cui, G., Chen, L., $\mathrm{Li}_{4} \mathrm{Ti}_{5} \mathrm{O}_{12}$-based energy conversion and storage systems: Status and prospects. Coord. Chem. Rev. 2017, 343, 139-184.

[7] Bruce, P. G., Martinet, S., Nanomaterials for rechargeable lithium batteries. Nanosci. Technol. 2016, 471-512.

[8] M.Thackeray, M., Thackeray, M. M., Manganese oxides for lithium batteries. Prog. Solid State Chem. 1997, 25, 1-71.

[9] Lee, M. J., Lee, S., Oh, P., Kim, Y., Cho, J., High performance $\mathrm{LiMn}_{2} \mathrm{O}_{4}$ cathode materials grown with epitaxial layered nanostructure for Li-Ion batteries. Nano Lett. 2014, 14, 993-999.

[10] Chen, C. H., Amine, K., Ionic conductivity, lithium insertion and extraction of lanthanum lithium titanate. Solid State Ionics 2001, 144, 51-57.

[11] Zheng, Z., Fang, H. Z., Liu, Z. K., Wang, Y., A fundamental stability study for amorphous $\mathrm{LiLaTiO}_{3}$ solid electrolyte. J. Electrochem. Soc. 2015, 162, A244-A248.

[12] Kamaya, N., Homma, K., Yamakawa, Y., Hirayama, M., Kanno, R., Yonemura, M., 
Kamiyama, T., Kato, Y., Hama, S., Kawamoto, K., Mitsui, A., A lithium superionic conductor. Nat. Mater. 2011, 10, 682-686.

[13] Yi, T. F., Yang, S. Y., Xie, Y., Recent advances of $\mathrm{Li}_{4} \mathrm{Ti}_{5} \mathrm{O}_{12}$ as a promising next generation anode material for high power lithium-ion batteries. J. Mater. Chem. A 2015, $3,5750-5777$.

[14] Wagemaker, M., Simon, D. R., Kelder, E. M., Schoonman, J., Ringpfeil, C., Haake, U., Lützenkirchen-Hecht, D., Frahm, R., Mulder, F. M., A kinetic two-phase and equilibrium solid solution in spinel $\mathrm{Li}_{4+\mathrm{x}} \mathrm{Ti}_{5} \mathrm{O}_{12}$. Adv. Mater. 2006, 18, 3169-3173.

[15] Wagemaker, M., Van Eck, E. R. H., Kentgens, A. P. M., Mulder, F. M., Li-ion diffusion in the equilibrium nanomorphology of spinel $\mathrm{Li}_{4+\mathrm{x}} \mathrm{Ti}_{5} \mathrm{O}_{12}$. J. Phys. Chem. B 2009, 113, 224-230.

[16] Ganapathy, S., Vasileiadis, A., Heringa, J. R., Wagemaker, M., The Fine Line between a Two-Phase and Solid-Solution Phase Transformation and Highly Mobile Phase Interfaces in Spinel $\mathrm{Li}_{4+\mathrm{x}} \mathrm{Ti}_{5} \mathrm{O}_{12}$. Adv. Energy Mater. 2017, 7, 1-12.

[17] Borghols, W. J. H., Wagemaker, M., Lafont, U., Kelder, E. M., Mulder, F. M., Size Effects in the $\mathrm{Li}_{4+}{ }_{4} \mathrm{Ti}_{5} \mathrm{O}_{12}$ Spinel. J. Am. Chem. Soc. 2009, 131, 17787-17792.

[18] Ge, H., Li, N., Li, D., Dai, C., Wang, D., Study on the theoretical capacity of spinel lithium titanate induced by low-potential intercalation. J. Phys. Chem. C 2009, 113, $6324-6326$.

[19] Hirayama, M., Kim, K., Toujigamori, T., Cho, W., Kanno, R., Epitaxial growth and electrochemical properties of $\mathrm{Li}_{4} \mathrm{Ti}_{5} \mathrm{O}_{12}$ thin-film lithium battery anodes. Dalt. Trans. 2011, 40, 2882-2887.

[20] Kim, K., Toujigamori, T., Suzuki, K., Taminato, S., Tamura, K., Mizuki, J., Hirayama, M., Kanno, R., Characterization of nano-sized epitaxial $\mathrm{Li}_{4} \mathrm{Ti}_{5} \mathrm{O}_{12}(110)$ film electrode for lithium batteries. Electrochemistry 2012, 80, 800-803.

[21] Zhong, Z., Ouyang, C., Shi, S., Lei, M., Ab initio studies on $\mathrm{Li}_{4+\mathrm{x}} \mathrm{Ti}_{5} \mathrm{O}_{12}$ compounds as anode materials for lithium-ion batteries. ChemPhysChem 2008, 9, 2104-2108.

[22] Ganapathy, S., Wagemaker, M., Nanosize storage properties in spinel $\mathrm{Li}_{4} \mathrm{Ti}_{5} \mathrm{O}_{12}$ explained by anisotropic surface lithium insertion. ACS Nano 2012, 6, 8702-8712.

[23] Hirayama, M., Shibusawa, T., Yamaguchi, R., Kim, K., Taminato, S., Yamada, N. L., Yonemura, M., Neutron reflectometry analysis of $\mathrm{Li}_{4} \mathrm{Ti}_{5} \mathrm{O}_{12} /$ organic electrolyte interfaces: characterization of surface structure changes and lithium intercalation properties. J. Mater. Res. 2016, 31, 3142-3150.

[24] Kleykamp, H., Phase equilibria in the Li-Ti-O system and physical properties of $\mathrm{Li}_{2} \mathrm{TiO}_{3}$. Fusion Eng. Des. 2002, 61-62, 361-366.

[25] Lakshmi Narayana, A., Dhananjaya, M., Guru Prakash, N., Hussain, O. M., Julien, C. M., Nanocrystalline $\mathrm{Li}_{2} \mathrm{TiO}_{3}$ electrodes for supercapattery application. Ionics (Kiel). 2017, 23, 3419-3428.

[26] Wang, Q., Guo, Q., Hu, Y., Li, B., High-quality spinel $\mathrm{LiCoTiO}_{4}$ single crystals with coexposed $\{111\}$ and $\{110\}$ facets: flux growth, formation mechanism, magnetic behavior and their application in photocatalysis. CrystEngComm 2016, 18, 6926-6933. 
[27] Wang, F., Wang, X., Chang, Z., Zhu, Y., Fu, L., Liu, X., Wu, Y., Electrode materials with tailored facets for electrochemical energy storage. Nanoscale Horizons 2016, 1, 272-289.

[28] Antczak, G., Ehrlich, G., Jump processes in surface diffusion. Surf. Sci. Rep. 2007, 62, 39-61.

[29] Mosa, J., Vélez, J. F., Reinosa, J. J., Aparicio, M., Yamaguchi, A., Tadanaga, K., Tatsumisago, M., $\mathrm{Li}_{4} \mathrm{Ti}_{5} \mathrm{O}_{12}$ thin-film electrodes by sol-gel for lithium-ion microbatteries. J. Power Sources 2013, 244, 482-487.

[30] Song, M. S., Kim, R. H., Baek, S. W., Lee, K. S., Park, K., Benayad, A., Is Li4Ti5 $\mathrm{O}_{12}$ a solid-electrolyte- interphase-free electrode material in Li-ion batteries? Reactivity between the $\mathrm{Li}_{4} \mathrm{Ti}_{5} \mathrm{O}_{12}$ electrode and electrolyte. J. Mater. Chem. A 2014, 2, 631-636.

[31] Rezvani, S. J., Gunnella, R., Witkowska, A., Mueller, F., Pasqualini, M., Nobili, F., Passerini, S., Cicco, A. Di, Is the Solid Electrolyte Interphase an Extra-Charge Reservoir in Li-Ion Batteries? ACS Appl. Mater. Interfaces 2017, 9, 4570-4576.

[32] Vasileiadis, A., de Klerk, N. J. J., Smith, R. B., Ganapathy, S., Harks, P. P. R. M. L., Bazant, M. Z., Wagemaker, M., Toward Optimal Performance and In-Depth Understanding of Spinel $\mathrm{Li}_{4} \mathrm{Ti}_{5} \mathrm{O}_{12}$ Electrodes through Phase Field Modeling. $A d v$. Funct. Mater. 2018, 28, 1-18.

[33] Fergusona, T. R., Bazant, M. Z., Nonequilibrium thermodynamics of porous electrodes. J. Electrochem. Soc. 2012, 159, A1967-A1985.

[34] Bazant, M. Z., Theory of chemical kinetics and charge transfer based on nonequilibrium thermodynamics. Acc. Chem. Res. 2013, 46, 1144-1160.

[35] Smith, R. B., Bazant, M. Z., Multiphase porous electrode theory. J. Electrochem. Soc. 2017, 164, E3291-E3310.

[36] Cunha, D. M., Hendriks, T. A., Vasileiadis, A., Vos, C. M., Verhallen, T., Singh, D. P., Wagemaker, M., Huijben, M., Doubling Reversible Capacities in Epitaxial $\mathrm{Li}_{4} \mathrm{Ti}_{5} \mathrm{O}_{12}$ Thin Film Anodes for Microbatteries. ACS Appl. Energy Mater. 2019, 2, 3410-3418.

[37] Wang, C., Wang, S., He, Y. B., Tang, L., Han, C., Yang, C., Wagemaker, M., Li, B., Yang, Q.-H., Kim, J.-K., Kang, F., Combining Fast Li-Ion Battery Cycling with Large Volumetric Energy Density. Grain Boundary Induced High Electronic and Ionic Conductivity in $\mathrm{Li}_{4} \mathrm{Ti}_{5} \mathrm{O}_{12}$ Spheres of Densely Packed Nanocrystallites. Chem. Mater. 2015, 27, 5647-5656.

[38] Verde, M. G., Baggetto, L., Balke, N., Veith, G. M., Seo, J. K., Wang, Z., Meng, Y. S., Elucidating the Phase Transformation of $\mathrm{Li}_{4} \mathrm{Ti}_{5} \mathrm{O}_{12}$ Lithiation at the Nanoscale. ACS Nano 2016, 10, 4312-4321.

[39] Ma, J., Hu, P., Cui, G., Chen, L., Surface and Interface Issues in Spinel $\mathrm{LiNi}_{0.5} \mathrm{Mn}_{1.5} \mathrm{O}_{4}$ : Insights into a Potential Cathode Material for High Energy Density Lithium Ion Batteries. Chem. Mater. 2016, 28, 3578-3606.

[40] Gummow, R. J., de Kock, A., Thackeray, M. M., Improved capacity retention in rechargeable 4 V lithium/lithium-manganese oxide (spinel) cells. Solid State Ionics 1994, $69,59-67$.

[41] Reddy, T. B., Linden, D., Linden's Handbook of Batteries. McGraw-Hill, New York 
2011.

[42] Ven, A. Van Der, Marianetti, C., Morgan, D., Ceder, G., Phase transformations and volume changes in spinel $\mathrm{Li}_{x} \mathrm{Mn}_{2} \mathrm{O}_{4}$. Solid State Ionics 2000, 135, 21-32.

[43] Thackeray, M. M., David, W. I. F., Bruce, P. G., Goodenough, J. B., Lithium Insertion into Manganese Spinels. Mater. Res. Bull. 1983, 18, 461-472.

[44] Hunter, J. C., Preparation of a new crystal form of manganese dioxide: $\lambda-\mathrm{MnO}_{2}$. J. Solid State Chem. 1981, 39, 142-147.

[45] Nitta, N., Wu, F., Lee, J. T., Yushin, G., Li-ion battery materials: Present and future. Mater. Today 2015, 18, 252-264.

[46] Luntz, A. C., Voss, J., Reuter, K., Interfacial Challenges in Solid-State Li Ion Batteries. J. Phys. Chem. Lett. 2015, 6, 4599-4604.

[47] Wang, K. X., Li, X. H., Chen, J. S., Surface and interface engineering of electrode materials for lithium-ion batteries. Adv. Mater. 2015, 27, 527-545.

[48] Myung, S. T., Lee, K. S., Kim, D. W., Scrosati, B., Sun, Y. K., Spherical core-shell $\mathrm{Li}\left[(\mathrm{Li} 0.05 \mathrm{Mn} 0.95){ }_{0.8}\left(\mathrm{Ni}_{0.25} \mathrm{Mn} 0.75\right)_{0.2}\right]_{2} \mathrm{O}_{4}$ spinels as high performance cathodes for lithium batteries. Energy Environ. Sci. 2011, 4, 935-939.

[49] Amos, C. D., Roldan, M. A., Varela, M., Goodenough, J. B., Ferreira, P. J., Revealing the Reconstructed Surface of $\mathrm{Li}\left[\mathrm{Mn}_{2}\right] \mathrm{O}_{4}$. Nano Lett. 2016, 16, 2899-2906.

[50] Jiao, F., Bao, J., Hill, A. H., Bruce, P. G., Synthesis of ordered mesoporous Li-Mn-O spinel as a positive electrode for rechargeable lithium batteries. Angew. Chemie - Int. Ed. 2008, 47, 9711-9716.

[51] Park, S. Bin, Shin, H. C., Lee, W. G., Cho, W. Il, Jang, H., Improvement of capacity fading resistance of $\mathrm{LiMn}_{2} \mathrm{O}_{4}$ by amphoteric oxides. J. Power Sources 2008, 180, 597601 .

[52] Yuan, Y., Amine, K., Lu, J., Shahbazian-Yassar, R., Understanding materials challenges for rechargeable ion batteries with in situ transmission electron microscopy. Nat. Commun. 2017, 8, 1-14.

[53] Lin, F., Markus, I. M., Nordlund, D., Weng, T. C., Asta, M. D., Xin, H. L., Doeff, M. M., Surface reconstruction and chemical evolution of stoichiometric layered cathode materials for lithium-ion batteries. Nat. Commun. 2014, 5, 3529.

[54] Lee, M. J., Lho, E., Bai, P., Chae, S., Li, J., Cho, J., Low-Temperature Carbon Coating of Nanosized $\mathrm{Li}_{1.015} \mathrm{Al}_{0.06} \mathrm{Mn}_{1.925} \mathrm{O}_{4}$ and High-Density Electrode for High-Power Li-Ion Batteries. Nano Lett. 2017, 17, 3744-3751.

[55] Kim, D. K., Muralidharan, P., Lee, H. W., Ruffo, R., Yang, Y., Chan, C. K., Peng, H., Huggins, R. A., Cui, Y., Spinel $\mathrm{LiMn}_{2} \mathrm{O}_{4}$ nanorods as lithium ion battery cathodes. Nano Lett. 2008, 8, 3948-3952.

[56] Bhandari, A., Bhattacharya, J., Review-Manganese Dissolution from Spinel Cathode: Few Unanswered Questions. J. Electrochem. Soc. 2017, 164, A106-A127.

[57] Hosono, E., Kudo, T., Honma, I., Matsuda, H., Zhou, H., Synthesis of single crystalline spinel $\mathrm{LiMn}_{2} \mathrm{O}_{4}$ nanowires for a lithium ion battery with high power density. Nano Lett. 2009, 9, 1045-1051. 
[58] Kim, J. S., Kim, K., Cho, W., Shin, W. H., Kanno, R., Choi, J. W., A truncated manganese spinel cathode for excellent power and lifetime in lithium-ion batteries. Nano Lett. 2012, 12, 6358-6365.

[59] Hirayama, M., Sonoyama, N., Ito, M., Minoura, M., Mori, D., Yamada, A., Tamura, K., Mizuki, J., Kanno, R., Characterization of Electrode/Electrolyte Interface with X-Ray Reflectometry and Epitaxial-Film $\mathrm{LiMn}_{2} \mathrm{O}_{4}$ Electrode. J. Electrochem. Soc. 2007, 154, A1065.

[60] Jiang, C., Tang, Z., Wang, S., Zhang, Z., A truncated octahedral spinel $\mathrm{LiMn}_{2} \mathrm{O}_{4}$ as highperformance cathode material for ultrafast and long-life lithium-ion batteries. J. Power Sources 2017, 357, 144-148.

[61] Sonoyama, N., Iwase, K., Takatsuka, H., Matsumura, T., Imanishi, N., Takeda, Y., Kanno, R., Electrochemistry of $\mathrm{LiMn}_{2} \mathrm{O}_{4}$ epitaxial films deposited on various single crystal substrates. J. Power Sources 2009, 189, 561-565.

[62] Hirayama, M., Ido, H., Kim, K., Cho, W., Tamura, K., Mizuki, J., Kanno, R., Dynamic Structural Changes at $\mathrm{LiMn}_{2} \mathrm{O}_{4} /$ Electrolyte Interface during Lithium Battery Reaction. $J$. Am. Chem. Soc. 2010, 132, 15268-15276.

[63] Suzuki, K., Kim, K., Taminato, S., Hirayama, M., Kanno, R., Fabrication and electrochemical properties of $\mathrm{LiMn}_{2} \mathrm{O}_{4} / \mathrm{SrRuO}_{3}$ multi-layer epitaxial thin film electrodes. J. Power Sources 2013, 226, 340-345.

[64] Gao, X., Ikuhara, Y. H., Fisher, C. A. J., Moriwake, H., Kuwabara, A., Oki, H., Kohama, K., Yoshida, R., Huang, R., Ikuhara, Y., Structural Distortion and Compositional Gradients Adjacent to Epitaxial $\mathrm{LiMn}_{2} \mathrm{O}_{4}$ Thin Film Interfaces. Adv. Mater. Interfaces 2014, 1, 1-10.

[65] Ikuhara, Y. H., Gao, X., Huang, R., Fisher, C. A. J., Kuwabara, A., Moriwake, H., Kohama, K., Epitaxial growth of $\mathrm{LiMn}_{2} \mathrm{O}_{4}$ thin films by chemical solution deposition for multilayer lithium-ion batteries. J. Phys. Chem. C 2014, 118, 19540-19547.

[66] Nakayama, N., Nozawa, T., Iriyama, Y., Abe, T., Ogumi, Z., Kikuchi, K., Interfacial lithium-ion transfer at the $\mathrm{LiMn}_{2} \mathrm{O}_{4}$ thin film electrode/aqueous solution interface. $J$. Power Sources 2007, 174, 695-700.

[67] Xia, H., Wan, Y., Yan, F., Lu, L., Manganese oxide thin films prepared by pulsed laser deposition for thin film microbatteries. Mater. Chem. Phys. 2014, 143, 720-727.

[68] Qiu, Y., Xu, G. L., Yan, K., Sun, H., Xiao, J., Yang, S., Sun, S. G., Jin, L., Deng, H., Morphology-conserved transformation: Synthesis of hierarchical mesoporous nanostructures of $\mathrm{Mn}_{2} \mathrm{O}_{3}$ and the nanostructural effects on Li-ion insertion/deinsertion properties. J. Mater. Chem. 2011, 21, 6346-6353.

[69] Alpen, U. V., Rabenau, A., Talat, G. H., Ionic conductivity in $\mathrm{Li}_{3} \mathrm{~N}$ single crystals. Appl. Phys. Lett. 1977, 30, 621-623.

[70] Kanno, R., Murayama, M., Lithium Ionic Conductor Thio-LISICON: The $\mathrm{Li}_{2} \mathrm{~S}-\mathrm{GeS}_{2}-\mathrm{P}$ ${ }_{2} \mathrm{~S}_{5}$ System. J. Electrochem. Soc. 2001, 148, 5-10.

[71] Mizuno, F., Hayashi, A., Tadanaga, K., Tatsumisago, M., New, highly ion-conductive crystals precipitated from $\mathrm{Li}_{2} \mathrm{~S}_{-} \mathrm{P}_{2} \mathrm{~S}_{5}$ glasses. Adv. Mater. 2005, 17, 918-921.

[72] Inaguma, Y., Chen, L., Itoh, M., Nakamura, T., High Ionic Conductivity in Lithium 
Lanthanum Titanate. Solid State Commun. 1993, 86, 689-693.

[73] Stramare, S., Thangadurai, V., Weppner, W., Lithium Lanthanum Titanates: A Review. Chem. Mater. 2003, 34, 3974-3990.

[74] Kim, S., Hirayama, M., Suzuki, K., Kanno, R., Hetero-epitaxial growth of $\mathrm{Li}_{0.17} \mathrm{La}_{0.61} \mathrm{TiO}_{3}$ solid electrolyte on $\mathrm{LiMn}_{2} \mathrm{O}_{4}$ electrode for all solid-state batteries. Solid State Ionics 2014, 262, 578-581.

[75] Aguesse, F., Roddatis, V., Roqueta, J., García, P., Pergolesi, D., Santiso, J., Kilner, J. A., Microstructure and ionic conductivity of LLTO thin films: Influence of different substrates and excess lithium in the target. Solid State Ionics 2015, 272, 1-8.

[76] Bohnke, O., Duroy, H., Fourquet, J. L., Ronchetti, S., Mazza, D., In search of the cubic phase of the $\mathrm{Li}+$ ion-conducting perovskite $\mathrm{La}_{2} / 3-\mathrm{L} \mathrm{Li}_{3 x} \mathrm{TiO}_{3}$ : Structure and properties of quenched and in situ heated samples. Solid State Ionics 2002, 149, 217-226.

[77] Mazza, D., Ronchetti, S., Bohnké, O., Duroy, H., Fourquet, J. L., Modeling Li-ion conductivity in fast ionic conductor $\mathrm{La}_{2 / 3-\mathrm{x}} \mathrm{Li}_{3 \times} \mathrm{TiO}_{3}$. Solid State Ionics $2002,149,81-88$.

[78] Ibarra, J., Várez, A., León, C., Santamaría, J., Torres-Martínez, L. M., Sanz, J., Influence of composition on the structure and conductivity of the fast ionic conductors La2/3${ }_{x} \mathrm{Li}_{3 x} \mathrm{TiO}_{3}(0.03 \leq x \leq 0.167)$. Solid State Ionics $2000,134,219-228$.

[79] Kim, S., Hirayama, M., Cho, W., Kim, K., Kobayashi, T., Kaneko, R., Suzuki, K., Kanno, R., Low temperature synthesis and ionic conductivity of the epitaxial $\mathrm{Li}_{0.17} \mathrm{La}_{0.61} \mathrm{TiO}_{3}$ film electrolyte. CrystEngComm 2014, 16, 1044-1049.

[80] Rivera, A., León, C., Santamaría, J., Várez, A., V’Yunov, O., Belous, A. G., Alonso, J. A., Sanz, J., Percolation-limited ionic diffusion in $\mathrm{Li}_{0.5-\mathrm{x}} \mathrm{Na}_{x} \mathrm{La}_{0.5} \mathrm{TiO}_{3}$ perovskites $(0 \leq \mathrm{x}$ $\leq 0.5)$. Chem. Mater. 2002, 14, 5148-5152.

[81] Kawai, H., Kuwano, J., Lithium Ion Conductivity of A-site deficient perovskite solid

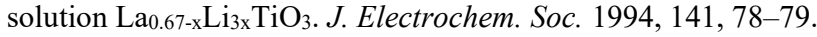

[82] Wei, J., Ogawa, D., Fukumura, T., Hirose, Y., Hasegawa, T., Epitaxial strain-controlled ionic conductivity in li-ion solid electrolyte $\mathrm{Li}_{0.33} \mathrm{La}_{0.56} \mathrm{TiO}_{3}$ thin films. Cryst. Growth Des. 2015, 15, 2187-2191.

[83] Ohnishi, T., Mitsuishi, K., Nishio, K., Takada, K., Epitaxy of $\mathrm{Li}_{3 x} \mathrm{La}_{2} / 3-\mathrm{x}$ TiO 3 films and the influence of la ordering on Li-ion conduction. Chem. Mater. 2015, 27, 1233-1241.

[84] Bohnke, O., Pham, Q. N., Boulant, A., Emery, J., Šalkus, T., Barré, M., H+/Li+ exchange property of $\mathrm{Li}_{3 \times \mathrm{La}_{2} / 3-\mathrm{TiO}}$ in water and in humid atmosphere. Solid State Ionics 2011, 188, 144-147.

[85] Lindström, H., Södergren, S., Solbrand, A., Rensmo, H., Hjelm, J., Hagfeldt, A., Lindquist, S. E., $\mathrm{Li}^{+}$ion insertion in $\mathrm{TiO}_{2}$ (anatase). 1. Chronoamperometry on CVD films and nanoporous films. J. Phys. Chem. B 1997, 101, 7710-7716.

[86] Funke, K., Debye-Hückel-Type Relaxation Processes in Solid Ionic Conductors: The Model. Solid State Ionics 1986, 183-190.

[87] McKee, R. A., A generalization of the Nernst-Einstein Equation for self-diffusion in high defect concentration solids. Solid State Ionics 1981, 5, 133-136.

[88] Kim, D. H., Kim, D. H., Jeong, Y. C., Seo, H. Il, Kim, Y. C., Lithium ion migration 
pathways in $\mathrm{Li}_{3 \times} \mathrm{La}_{2 / 3-x} \square_{1 / 3-2 x} \mathrm{TiO}_{3}$. Ceram. Int. 2012, 38S, S467-S470.

[89] Moriwake, H., Gao, X., Kuwabara, A., Fisher, C. A. J., Kimura, T., Ikuhara, Y. H., Kohama, K., Tojigamori, T., Ikuhara, Y., Domain boundaries and their influence on Li migration in solid-state electrolyte (La,Li) $\mathrm{TiO}_{3}$. J. Power Sources 2015, 276, $203-207$.

[90] Liu, G. Q., Wen, L., Liu, Y. M., Spinel $\mathrm{LiNi}_{0.5} \mathrm{Mn}_{1.5} \mathrm{O}_{4}$ and its derivatives as cathodes for high-voltage Li-ion batteries. J. Solid State Electrochem. 2010, 14, 2191-2202.

[91] Zhu, Y., He, X., Mo, Y., First principles study on electrochemical and chemical stability of solid electrolyte-electrode interfaces in all-solid-state Li-ion batteries. J. Mater. Chem. A 2016, 4, 3253-3266.

[92] Richards, W. D., Miara, L. J., Wang, Y., Kim, J. C., Ceder, G., Interface Stability in Solid-State Batteries. Chem. Mater. 2016, 28, 266-273.

[93] Christian Julien, Comparative Issues of Cathode Materials for Li-Ion Batteries Christian. Inorganics 2014, 2, 132-154.

[94] Zheng, H., Straub, F., Zhan, Q., Yang, P. L., Hsieh, W. K., Zavaliche, F., Chu, Y. H., Dahmen, U., Ramesh, R., Self-assembled growth of $\mathrm{BiFeO}_{3}-\mathrm{CoFe}_{2} \mathrm{O}_{4}$ nanostructures. Adv. Mater. 2006, 18, 2747-2752. 


\title{
Chapter 3
}

\section{Morphology Evolution during Li-based VAN Growth}

\begin{abstract}
Ceramics-based nanocomposites is a rapidly evolving research area, as they are currently being used in a wide range of applications. Epitaxial vertically aligned nanocomposites (VANs) offer promising advantages over conventional planar multilayers as key functionalities are tailored by the strong coupling at their vertical interfaces. However, limited knowledge exists of which materials systems are compatible in composite films and which types of structures are optimal for a given functionality. No lithium-based VANs have yet been explored for energy storage, while 3D solid-state batteries offer great promises for enhanced energy and power densities. Although solidon-solid Kinetic Monte-Carlo simulation (KMCS) models of VAN growth have previously been developed, phase separation was forced into the systems by limiting hopping directions and/or tuning activation energies for hopping. The focus of this chapter is the influence of temperature and deposition rate on the morphology evolution of lithium-based VANs, consisting of promising $\mathrm{LiMn}_{2} \mathrm{O}_{4}$ cathode and $\mathrm{Li}_{0.5} \mathrm{La}_{0.5} \mathrm{TiO}_{3}$ electrolyte, by applying a KMCS model with activation energies for hopping obtained experimentally and with minimum restrictions for hopping directions. Although the model considers only the kinetic processes and disregards the thermodynamics of growth that would determine the shape of pillars within the matrix, the trends in pillar size and distribution within the simulated VANs are in good agreement with experiments. This provides an elegant tool to predict the growth of VAN materials, as the experimental activation energies and higher degrees of freedom for hopping result in a more realistic and low computational cost model to obtain accurate simulations of VAN materials.
\end{abstract}

Based on the publication:

D.M. Cunha, C.M. Vos, T.A. Hendriks, D.P. Singh, M. Huijben, Morphology Evolution during LithiumBased Vertically Aligned Nanocomposite Growth. ACS Appl. Mater. Interfaces. 2019, 11, 47, 44444-44450. 


\subsection{Introduction}

Since no lithium-based VANs have yet been explored for energy storage, in this chapter a model was developed to predict the formation of these structures. Nanocomposites have attracted great interest over the last decades due to the presence of enhanced functional material properties induced by confinement of the structural dimensions.[1] Ceramicbased nanocomposites is one of the most rapidly evolving research areas, [2] as they are currently being used in a wide range of applications, such as motor engines, heat exchangers, power plants, and air-/spacecraft technology. However, accurate control of the distribution and orientation of the nanoparticles within the matrix material is often limited, or even impossible. Detailed knowledge on the alignment of nanostructures through self-assembly is very well studied in organic systems, [3] but remains a rather unexplored territory for inorganic nanocomposites.

In parallel to planar heterointerfaces, vertical heteroepitaxial nanocomposite thin films have been developed in the past decade as a new platform for creating self-assembled device architectures and multifunctionalities.[4,5] Such epitaxial vertically aligned nanocomposites (VANs) offer promising advantages over conventional planar multilayers as key functionalities are tailored by the strong coupling between the two phases at their interfaces, such as strain-enhanced ferroelectricity and multiferroics, [6,7] ferromagnetism,[8] magnetoresistance,[9] electronic transport,[10]and coupled dielectric and optical effects.[11]

Epitaxial VANs are self-assembled through physical vapor deposition, without control of the deposition sequence, as is required for planar multilayer films. For epitaxially directed self-assembly, it is desirable that one phase in the film is structurally wellmatched to the substrate such that it nucleates, grows epitaxially and forms the host matrix. The second phase (epiphyte) epitaxially aligns with the matrix phase. Depending on surface energy considerations and the relative concentrations of the two components in the film, the epiphyte phase may or may not seed its growth on the substrate. The host and epiphyte can both be chosen to be active phases whose functional properties are of interest, and where the phases interact with each other via strain- or charge-coupling at the interfaces. Dimensional tunability has been demonstrated, in which various nanopillar shapes and dimensions $(2-200 \mathrm{~nm})$ have been achieved in specific material systems. [4,5]

There is currently limited knowledge of which materials systems are compatible with one another in composite films and which types of structures are optimal for a given functionality.[12] To be able to design optimal VAN structures the current knowledge gap on fundamental understanding of the growth mechanisms during composite nanostructure formation must be filled. Detailed simulation of the VAN growth mechanism would provide important insight into the compositional, structural, and 
processing factors that influence the achievable VAN structures. Solid-on-solid Kinetic Monte-Carlo simulation (KMCS) models of vertical nanocomposite growth have previously been developed to study the influence of temperature,[13-16] deposition rate[14,17] and composition ratio[13,15] on the VAN morphology. Although the studied models result in good approximation of experimental results, they force phase separation into the nanocomposite system[13-17] by limiting the permitted hopping orientations, and/or by tuning the activation energies for hopping to fit experimental results.

Although various epitaxial VANs have been studied in the last decade, [4,5] the successful realization of two-phase epitaxial VANs has remained limited to specific material combinations: e.g. ferroelectrics $\left(\mathrm{BaTiO}_{3}, \mathrm{BiFeO}_{3}, \mathrm{PbTiO}_{3}\right)$ with ferromagnets $\left(\mathrm{CoFe}_{2} \mathrm{O}_{4}, \mathrm{NiFe}_{2} \mathrm{O}_{4}, \mathrm{MgFe}_{2} \mathrm{O}_{4}, \mathrm{La}_{0.7} \mathrm{Sr}_{0.3} \mathrm{MnO}_{3}, \mathrm{Fe}_{3} \mathrm{O}_{4}\right) ; \mathrm{BiFeO}_{3}$ ferroelectric with $\mathrm{LaFeO}_{3}$ antiferromagnet; and $\mathrm{ZnO}$ insulator with $\mathrm{La}_{0.7} \mathrm{Sr}_{0.3} \mathrm{MnO}_{3}$ ferromagnet. No lithium-based VANs have yet been explored for energy storage, while 3D solid-state batteries offer great promises for enhanced energy and power densities.[18,19] The potential advantages of VAN-based lithium-ion batteries are: larger electrode/electrolyte contact area leading to higher (dis)charge rates; short path lengths for both electronic and Li ion transport leading to higher lithium-ion flux (also allowing the usage of materials with low electronic or ionic conductivity); better accommodation of the strain during lithium insertion/extraction; and interface reactions not available in bulk materials.

The focus of this chapter is to analyze the influence of temperature and deposition rate on the morphology evolution of lithium-based VANs by applying a KMCS model with activation energies for hopping obtained experimentally and with minimum restrictions for hopping directions. The combination of KMCS and reflective high energy electron diffraction (RHEED) measurements provides an elegant tool to predict the growth of VAN materials. Although the model considers only the kinetic processes and disregards the thermodynamics of growth that would determine the exact shape of the pillars within the matrix, the trends in pillar size and distribution within the simulated VANs are in good agreement with experiments. It is shown that experimental activation energies and higher degrees of freedom for hopping result in a more realistic and low computational cost model that can be used to obtain accurate simulated results of VAN materials. Furthermore, we demonstrate the successful growth of lithium-based VANs consisting of the promising $\mathrm{LiMn}_{2} \mathrm{O}_{4}(\mathrm{LMO})$ cathode and $\mathrm{Li}_{0.5} \mathrm{La}_{0.5} \mathrm{TiO}_{3}$ (LLTO) electrolyte materials.

\subsection{PLD growth and structural analysis of VAN thin films}

To validate the results obtained from the simulations, VAN films were deposited by PLD and the morphology analyzed by AFM. Spinel LMO is a high voltage cathode material[20] exhibiting a spinel structure with lattice parameter of $a=8.245 \AA$, while LLTO is a high ionic conducting electrolyte [21] with a perovskite structure $(a=$ 
$3.904 \AA$ ), making them an interesting combination, similar to previous successful spinelperovskite VAN formations.[6,22] The films were grown from a sintered $67 \%$ $\mathrm{La}_{0.5} \mathrm{Li}_{0.5} \mathrm{TiO}_{3}+33 \% \mathrm{LiMn}_{2} \mathrm{O}_{4}(30 \mathrm{wt} \%$ excess $\mathrm{Li})$ target at an oxygen pressure of 0.2 mbar within the temperature range $700-900{ }^{\circ} \mathrm{C}$. A $\mathrm{KrF}$ excimer laser was used operating at $248 \mathrm{~nm}, 0.5-50 \mathrm{~Hz}$ and a laser energy fluence of $2.3 \mathrm{~J} . \mathrm{cm}^{-2}$ resulting in a growth rate of $\sim 0.15 \AA$ Apulse. The STO substrates ( $a=3.905 \AA$ )[23] were annealed in a tube-oven before the deposition at $950{ }^{\circ} \mathrm{C}$ for $90 \mathrm{~min}$ in an oxygen flow of $150 \mathrm{~L} / \mathrm{h}$. After deposition, the films were cooled down to room temperature in an oxygen pressure of 0.2 mbar at a rate of $10{ }^{\circ} \mathrm{C} / \mathrm{min}$. X-ray diffraction (XRD) measurements were performed on a PANalytical X'Pert PRO diffractometer, with a PIXcel1D detector and a $\mathrm{Cu}$ source $(\lambda=$ $1.5406 \AA$ A). Tapping-mode AFM was performed in air on a Bruker ICON Dimension Microscope. The size and distribution of the structures measured by AFM were obtained with Gwyddion, where pillar areas under $5 \mathrm{~nm}^{2}$ were neglected considering that it represents monomers on the surface. A Zeiss Merlin HRSEM was used for the scanning electron microscopy (SEM) images.

(a)

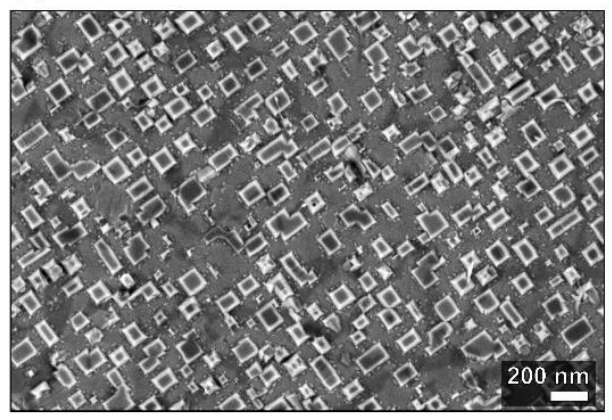

(b)

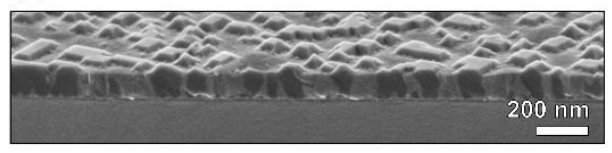

(c)
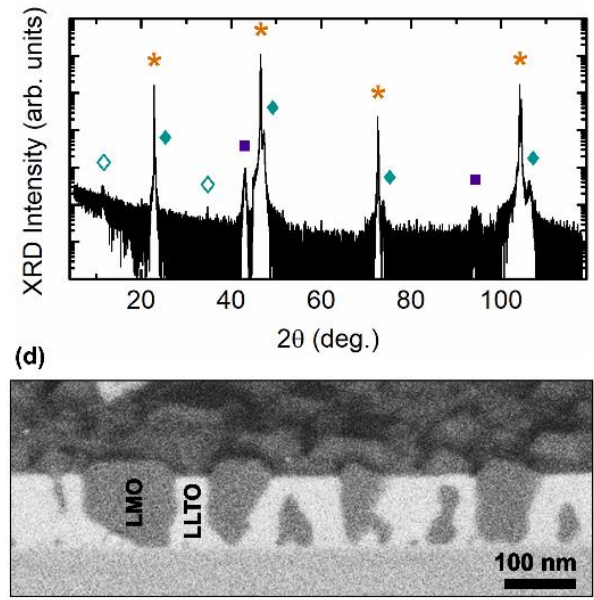

Figure 3-1. (a) Top-view and (b) cross-sectional SEM images of a nanocomposite thin film composed of LMO pillars embedded in LLTO matrix. (c) X-ray diffraction analysis of the VAN film, in which LLTO peaks are shown by $\diamond$, LMO by $\boldsymbol{\square}$, and the STO substrate peaks by $\star$, whereas minor contributions of the tetragonal LLTO phase are indicated by $\diamond$. (d) Cross-sectional Energy selective Backscattered SEM image showing the compositional contrast.

The successful phase separation of both materials into a nanopillar-matrix structure within the nanocomposite can be observed in the SEM images, Figure 3-1a and Figure $3-1 b$, while the purity and crystallinity of both specific phases are confirmed by XRD, Figure 3-1c. The out-of-plane (100) crystal orientations of both LMO and LLTO phases within the VAN films are aligned with the orientation of the STO substrate. The LMO 
and LLTO peaks show the presence of highly crystalline oriented spinel and perovskite structures, in a good agreement with previous studies of individual LMO or LLTO thin films grown on STO(100) substrates.[24,25] The small extra peaks suggest the presence of minor contributions of the tetragonal LLTO $\left(\mathrm{Li}_{0.56} \mathrm{La}_{0.33} \mathrm{TiO}_{3}\right)$, which could be randomly distributed throughout the LLTO matrix or located at specific interfaces. This minority tetragonal LLTO phase will be studied in detail in follow-up research. Also, the in-plane orientations of both cubic LMO and LLTO phases are aligned to the cubic substrate as confirmed by detailed XRD analysis (more details in Chapter 4), resulting in square LMO nanopillars rotated $45^{\circ}$ with respect to the (010) in-plane direction of both the perovskite LLTO matrix and STO substrate. Furthermore, phase separation was also confirmed by energy selective backscattered (ESB) SEM analysis, where contrast is determined by compositional differences (Figure 3-1d), leading to a much brighter LLTO matrix due to the presence of the heavy La ions.

\subsection{Modelling Self-Assembled Growth}

\subsubsection{Kinetic Monte Carlo Simulation}

Monte Carlo refers to a broad class of algorithms that solve problems using random numbers. They first emerged in the late 1940's and 1950's as electronic computers came into use,[26] and the name refers to the random nature of the gambling at Monte Carlo, Monaco. In the 1960's, different kinds of Monte Carlo algorithm were developed for evolving systems dynamically from state to state. The earliest application of this approach for an atomistic system was for radiation damage annealing, reported by Beeler et alii.[27] Over the next 20 years, there were developments and applications in other areas as surface adsorption, diffusion and growth,[28-31] which is applied here, and in statistical physics.[32] In the 1990's the terminology for this approach settled in as kinetic Monte Carlo. The popularity and range of applications of kinetic Monte Carlo (KMC) has continued to grow and $\mathrm{KMC}$ is now a common tool for studying the growth of materials, as stated in the introductory section. An appealing property of KMC is that it can, in principle, give the exact dynamical evolution of a system, although this ideal is virtually never achieved, or even attempted.

To accurately simulate atomic diffusion on a surface, KMC models applies the notion of infrequent-events. An infrequent-event system is one in which the dynamics is characterized by occasional transitions from one state to another, with long periods of relative inactivity between these transitions. Although the infrequent-event designation is fairly general (and hence also the possible applications of KMC), for simplicity the discussion is restricted to the case where each event corresponds to a single hop, and the 
long time between transitions arises because the system must overcome an energy barrier to get from one state to another. This is an appropriate description for most solid-state atomistic systems. The key property of an infrequent-event system in a state is that each event is disconnected from previous events. Then, for each possible event, there is a rate constant $k_{i j}$ that characterizes the probability, per unit time, that it permutes to that state $j$, and these rate constants are independent of what state preceded state $i$. This characteristic, that the transition probabilities for exiting state $i$ does not have influence from the history prior to entering state $i$, is the defining property of a Markov chain.[33] Thus, during each short increment of time, it has the same probability of finding an hopping path as it had in the previous increment of time. This gives rise to a first-order process with exponential decay statistics.

For each of the $M$ hopping possibilities there is an object with a length equal to the hopping rate $k_{i j}$ for that hopping event. The cumulative sum of these objects is defined as $k_{\text {tot }}$. To select a hopping event, a single random position along the length of this stack of objects is chosen. This procedure gives a probability of choosing a pathway that is proportional to the rate constant for that pathway. To advance the simulation time, a random number is drawn from the exponential distribution for the rate constant $k_{t o t}$. Note that the time advance does not depend on which event is chosen as the hopping time depends only on the total hopping rate. Once the system is in the new state, the list of events and rates is updated, and the procedure is repeated. The mathematical formalism that rule the Solid-on-Solid KMC algorithm will be presented in Section 3.3.2.

Usually in KMC simulations, the atoms in the system are mapped onto a lattice and each hopping event will move one atom to a new unique position in the grid. Lattice mapping makes it easy to exploit locality in determining rates, where only the atoms near the atom of interest affect the rate constant for any change for that atom. The rates computed in this way will form a catalog,[31] which can be used to search for the rates needed for every state the system may achieve. Finally, the locality imposed by this ratecatalog approach has the benefit that updating the list of rates after a move has been accepted requires only fixed amount of work, rather than work scaling as the number of atoms in the entire system. Where the rate is specified by a barrier height and a preexponential factor, an easy simplification is to assume that the barrier height can be approximated by additive interactions.

As mentioned before KMC can, in principle, give the exact state-to-state dynamics for a system. This assumes that a complete rate catalog has been generated, containing an accurate rate constant for every hopping possibility. The real dynamical evolution of a system, however, will often incorporate unexpected and complex pathways, not known beforehand. Because these pathways are firstly unknown, they will not be included in the rate catalog, and hence will not be accounted for during the KMC simulation. A classic example can be found on the field of surface diffusion. Until 15 years ago, diffusion of an adatom on the simple fcc(100) surface was assumed to occur by the adatom hopping 
from one site to the next. In 1990, however, calculations showed that the primary diffusion pathway on $\mathrm{Al}(100)$ actually involves the exchange event, in which the adatom plunges into the surface, pushing a substrate atom up into the second nearest neighbor binding site.[34] Thus, a KMC simulation of $\mathrm{Al}$ adatoms on a $\mathrm{Al}(100)$ surface, using a rate catalog built assuming hop events only, would give a seriously flawed description of the diffusion dynamics. More recently, there have been many examples of unexpected surface and bulk diffusion mechanisms.[35-38] In some cases, the discovered mechanisms are so complex that it would not be easy to incorporate them into a $\mathrm{KMC}$ rate catalog, even after the existence of the pathway is known.

The total simulation time achieved in a KMC simulation is strongly system dependent. Each KMC step advances the system by a time (on average) no greater than the inverse of the fastest hopping rate. This rate depends exponentially on the barrier height divided by the temperature, and the size of the lowest barrier can change as the system evolves. However, to get some sense of what is possible, present-day computers can take roughly $10^{10}$ steps in a few hours of computer time. If assumed that for every atom there is one fast hopping direction with a fixed lowest barrier $E_{a}$ and a prefactor of $10^{13}$, then a simulation time of $10^{10} /\left(10^{-13} \exp \left(\mathrm{E}_{\mathrm{a}} / \mathrm{k}_{\mathrm{B}} \mathrm{T}\right)\right)$ is achieved. For $\mathrm{E}_{\mathrm{a}}=0.5 \mathrm{eV}$, this gives a total simulation time with a factor of $10^{14} \mathrm{~s}$ at $\mathrm{T}=300 \mathrm{~K}, 10^{18} \mathrm{~S}$ at $\mathrm{T}=600 \mathrm{~K}$ and $10^{20} \mathrm{~s}$ at $\mathrm{T}=$ 1000K.[39]

Kinetic Monte Carlo is a very powerful and general method. Given a set of rate constants connecting states of a system, KMC offers a way to propagate dynamically correct trajectories through the state space. The type of system, as well as the definition of a state, is fairly arbitrary, provided it is appropriate to assume that the system will make first-order transitions among these states. If the rate catalog is constructed properly, the easily implemented KMC dynamics can give exact state-to-state evolution of the system. However, this ideal is virtually never realizable, primarily to the fact that there are reaction pathways in the system that are not expected. Despite these limitations, KMC remains a powerful approach available for making dynamical predictions at the mesoscale. Moreover, even in situations where a more accurate simulation would be feasible, the extreme efficiency of KMC makes it ideal for rapid scans over different conditions, for example, and for model studies, as will be described in the following sections.

\subsubsection{KMCS model with experimental activation energies and higher degrees of freedom for hopping}

For the KMCS modeling a 3-dimensional $128 \times 128 \times Z$ grid, was used to represent the cubic (100) surface of STO, where $\mathrm{Z}$ is the height. In these simulations the PLD growth process is simplified, as only deposition and diffusion of singular entities is allowed, 
depicted in Figure 3-2a. Typically, these entities are atoms when simulating growth of metals. However, in the case of oxides deposition and diffusion of complete unit cell blocks are simulated, which are referred here as adatoms. The deposition of material is simulated by instantaneously generating adatoms on randomly chosen locations on the grid. One adatom represents one unit cell of the LLTO perovskite crystal structure or 1/8th unit cell of the LMO spinel crystal structure. The number of adatoms deposited each pulse is $1 / 25$ th of the size of the grid, based on the RHEED results for the LLTO growth rate, shown in Figure 3-2b. No desorption is allowed.

After each pulse, the deposited material can diffuse over the surface, which is modeled as the hopping of adatoms, one at a time, to available surrounding coordinates (Figure 3-2a). Periodic boundary conditions were used, and hopping is only possible if a site is unoccupied and if there is a path available to it. Hopping continues until a new pulse arrives, at which moment the location of the old adatoms are fixed. The hopping rate of the adatoms is defined as:

$$
k_{i}=k_{0} e^{\left(-\frac{E_{D, i}}{k_{B} T}\right)}
$$

Where $k_{i}$ is the hopping rate of unit cell $i, k_{0}$ is the hop attempt frequency[13] $\left(\mathrm{k}_{0}=10^{12}\right.$ $\left.\mathrm{s}^{-1}\right), E_{D, i}$ is the activation energy for diffusion, $k_{B}$ is the Boltzmann constant and $T$ is the absolute temperature. The activation energy $E_{D}$ was calculated considering the sum of the interaction energies between the adatom and all occupied neighboring positions:

$$
E_{D}=E_{S}+\sum_{j=1}^{J} n_{j} \times E_{j}
$$

Where $E_{S}$ is a static contribution[41] $]^{\dagger}$ independent of neighboring sites, $n_{j}$ is the number of neighbors of type $j$ and $E_{j}$ is the interaction energy of the adatom with this neighbor. Only interactions between face-sharing adatoms are considered and long-range interactions are ignored. Hopping can occur both in the 6 cubic and 12 diagonal directions. For diagonal hops (step-down or step-up) the Ehrlich-Schwoebel barrier ( $\mathrm{E}_{\mathrm{ES}}$ ) is present due to the decreased coordination between a surface diffusing adatom and the substrate. An $E_{E S}$ value of $0.15 \mathrm{eV}$ was used in good agreement with previous studies for various material systems.[42-44]

$\dagger$ The static contribution $E_{S}$ is the surface energy, or the activation energy for hopping of a free adatom. The term was introduced by Ratsch et al.,[40] and in the model sets the number of hops per pulse. Lower $E_{S}$ increase the number of hops per pulse, increasing the computational time for the KMC simulation. 
(a)

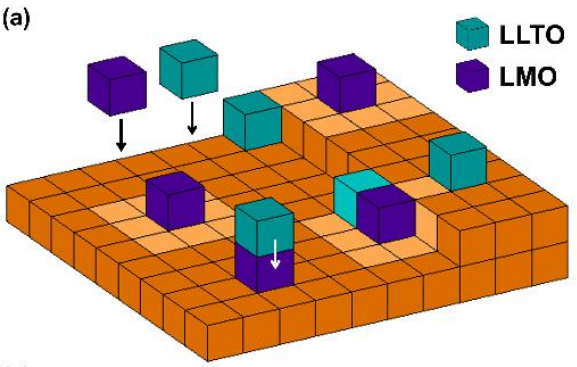

(c)

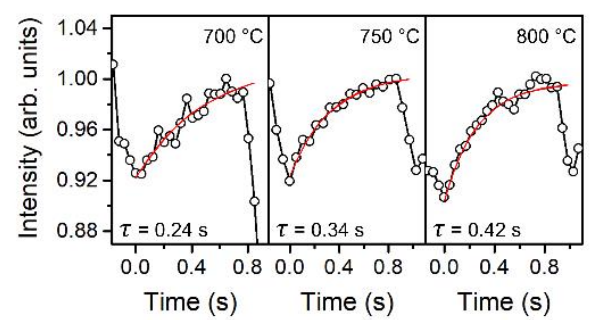

(1)

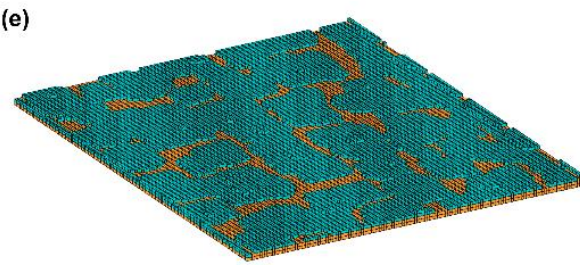

(b)

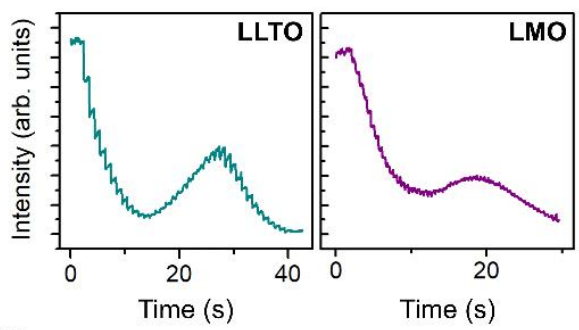

(d)
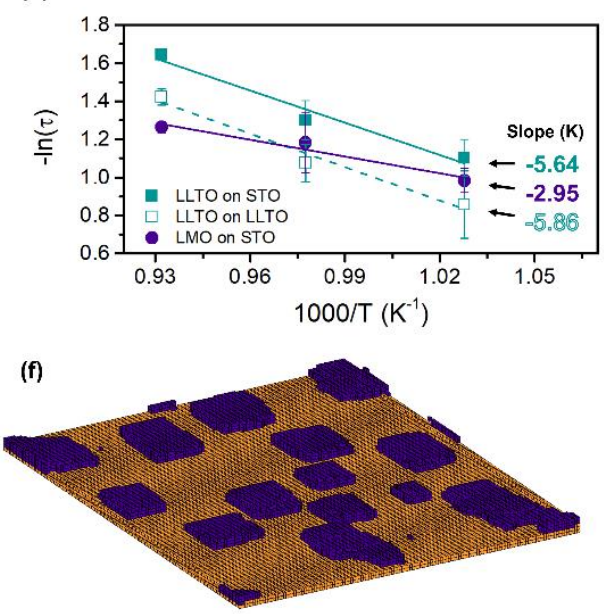

Figure 3-2. (a) Schematic of the KMCS model, representing the deposition and diffusion of both phases, LMO (purple) and LLTO (blue), on a STO (orange) surface. The bright areas indicate possible hopping positions for LMO adatoms on the surface. Note that adatoms that could lead to floating adatoms (indicated by the white arrow) have no possible hopping direction. (b) RHEED intensity oscillations during PLD growth of individual LLTO and LMO thin films on STO substrates. (c) Exponential fitting of the specular spot RHEED intensity variation during a single laser pulse for LLTO growth on LLTO surface at different temperatures. (d) Arrhenius plot of the RHEED relaxation time dependence on the growth temperatures for several combinations: LLTO on STO, LLTO on LLTO and LMO on STO. (e,f) KMC simulation showing the wetting and non-wetting behavior of LLTO and LMO thin films on a STO surface, based on 12 deposition pulses using the activation energies for hopping as obtained by RHEED analysis. 
To perform a hopping event a random number, $r(0<r<1)$, is generated and the adatom with hopping probability closer to $r \cdot K$ is chosen to hop, where $K$ is the cumulative sum of hopping probabilities. Adatoms with a higher hopping rate have a higher probability of being chosen.

After an adatom is selected, the $E_{E S}$ barrier determines the probability of diagonal movement. After each hop, the location of the adatoms in the $(\mathrm{X}, \mathrm{Y}, \mathrm{Z})$ matrix is updated, as well as the hopping rates that may have changed, and a new adatom is selected. The time of the simulation is updated after each hop using $\Delta t=-\frac{\log (r)}{K}$.[45] Once a simulation time has passed equal to $1 / f$, where $f$ is the deposition frequency, a new pulse of material arrives, and the simulation steps begin anew.

The time constant obtained from Figure 3-2c is a measure of the mobility of the diffusing adatoms, defined as:

$$
\ln (\tau)=\ln \left(l_{D}^{2} / v a^{2}\right)+\mathrm{E}_{\mathrm{A}} / \mathrm{k}_{\mathrm{B}} \cdot 1 / \mathrm{T}
$$

Where $l_{D}$ is the diffusion length, $v$ is the diffusion attempt frequency and $a$ is the characteristic jump distance. Therefore, using the obtained relaxation times $\tau$, an Arrhenius plot can be drawn (Figure 3-2d), where the slope is used to calculate the activation energy $E_{A}$ for each material combination.[46] From the initial growth of the

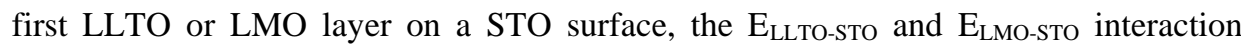
energies were determined, while subsequent thin film growth resulted in values for $\mathrm{E}_{\text {LLTO- }}$

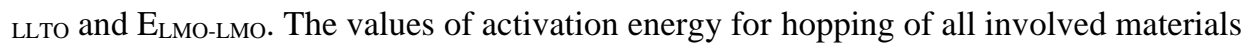
combinations are listed in Table 3-1. The combined effect of all physical interactions between the materials is reflected in this single value of the activation energy for diffusion. Considering how these values are applied in the KMCS model, a high value indicates favorable interactions, resulting in a wetting behavior, while a low value corresponds to a nonwetting behavior.

Table 3-1. Activation energies obtained by RHEED (in eV) and constants used in the KMC model.

\begin{tabular}{cccccccc}
\hline $\mathbf{k}_{\mathbf{0}}$ & $\mathbf{E}_{\mathbf{S}}$ & $\mathbf{E}_{\mathrm{ES}}$ & $\mathbf{E}_{\text {LMo-sto }}$ & $\mathbf{E}_{\text {LMO-LMO }}$ & $\mathbf{E}_{\text {LLTO-LMo }}$ & $\mathbf{E}_{\text {LLTO-STO }}$ & $\mathbf{E}_{\text {LLTO-LLTO }}$ \\
\hline $10^{12}$ & 1.0 & 0.15 & $0.25 \pm 0.01$ & $0.51 \pm 0.01$ & $0.25 \pm 0.01$ & $0.49 \pm 0.01$ & $0.51 \pm 0.01$ \\
\hline
\end{tabular}

The values for $\mathrm{E}_{\text {LLto-sto }}$ and $\mathrm{E}_{\text {LLTO-LLTo }}$ are similar, indicating similar favorable interactions, that can be explained by their identical perovskite crystal structure. The value for $\mathrm{E}_{\mathrm{LMO}-\mathrm{STO}}$ is significantly lower, indicating reduced wetting behavior for a spinel layer on a perovskite surface. Note that, by using these values, the (non)wettability is only implicitly added to the model, which successfully reproduced the wettability for both 
LLTO and LMO phases on STO surfaces, as shown in Figure 3-2e and Figure 3-2f, respectively. Due the Volmer-Weber growth nature of (100)-oriented LMO,[24] $\mathrm{E}_{\mathrm{LMO}}$

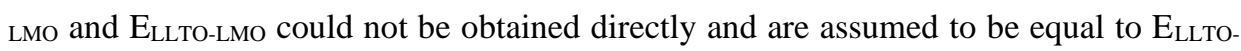
LLTo and E EMO-STo, respectively, since similar interactions are expected. The obtained activation energies are used in the KMC simulation to model the VAN formation at different growth temperatures.

\subsection{Comparison between experimental and simulation results}

Figure 3-3 shows KMC simulations for the VAN surface after 300 pulses of the nanocomposite growth at a $20 \mathrm{~Hz}$ deposition rate in the temperature range of $700-900{ }^{\circ} \mathrm{C}$. The KMCS results are in good agreement with the variation in VAN formation in the experiments as shown by the AFM images in Figure 3-3. The LMO and LLTO components phase-separate into well-defined and evenly spaced nanostructures. The KMCS model does not incorporate anisotropic interaction energies to reflect different crystal facets and disregards the thermodynamic processes for energy reduction at the interfaces between the different material phases, i.e. nanopillar and matrix. The two components show some intermixing because the interaction energies are relatively close, while increasing the difference between these values will lead to stronger phase separation.[47] However, the simulated vertical nanostructures are qualitatively similar to those described in previous nanocomposite studies.[13,15,41,47,48]
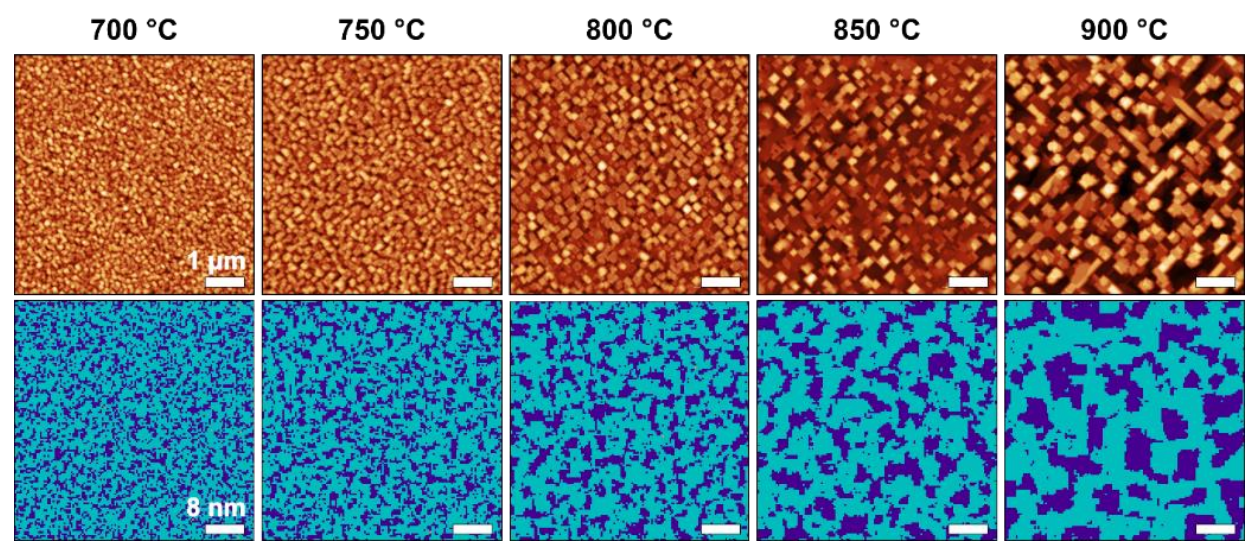

Figure 3-3. AFM images (top) and KMCS results (bottom) for the LMO-LLTO nanocomposite growth at a deposition rate of $20 \mathrm{~Hz}$ for different growth temperatures.

The cross-sectional SEM analysis is shown in Figure 3-4, with the experimental VAN thin films for different deposition rates alongside the 3D view of the KMCS results with the same parameters. The formation of the LMO nanopillars within the LLTO matrix can be observed as they are connected to the underlying STO substrate and grow through the 
full thickness of the VAN film. The vertical pillars formed at $2 \mathrm{~Hz}$ exhibit a larger diameter as compared to those grown at $8 \mathrm{~Hz}$. However, for a deposition rate of $0.5 \mathrm{~Hz}$ the LMO phase does not form vertical structures but grows in larger, three-dimensional agglomerates dispersed in the LLTO matrix. Notably, many LMO structures are not well connected to the STO substrate, and instead rest on a LLTO region in many locations.
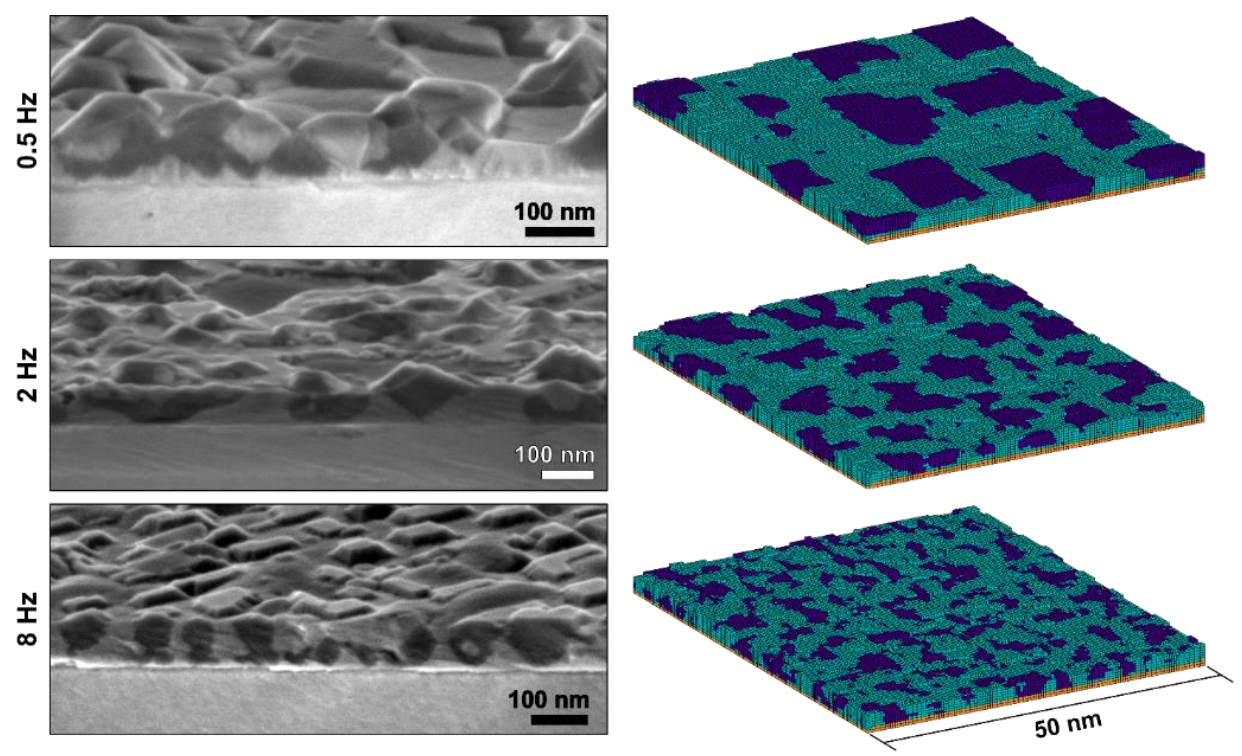

Figure 3-4. Cross-sectional SEM analysis (left) and 3D view of KMCS results (right) for the LMO-LLTO nanocomposite growth for different deposition rates $(0.5,2$ and $8 \mathrm{~Hz})$ at $800{ }^{\circ} \mathrm{C}$.

It can be clearly observed that a lower growth rate does not favor the growth of nanopillars even though particles have more time to hop over the surface to find their own species. It is expected that the formation of pillars depends on the islands that are formed in the initial stage of growth.[13,14] The diffusion length of the two components plays a critical role in the resulting island size,[47] and therefore the expected nanopillar formation. The diffusion length is given by:

$$
l_{D}=\sqrt{D \tau}
$$

Where $\tau$ is the diffusion time and $D$ is the diffusion coefficient, obtained by:

$$
D=v a^{2} e^{\left(-\frac{E_{A}}{k_{B} T}\right)}
$$


At low frequencies the long LMO diffusion length leads to the growth of a few larger islands. For higher frequencies the diffusion length rapidly drops, resulting in the growth of many smaller islands on the surface. For such higher frequencies, the diffusion length of both LMO and LLTO species becomes comparable, and vertically aligned structures are formed. This limits the pillar diameter to about $90 \mathrm{~nm}$ for VAN growth at higher frequencies, in good agreement with experimental results in previous studies.[22]

A quantitative analysis was performed considering the number density of pillars over the VAN film surface. Figure 3-5a and Figure 3-5b show the comparison of the number density between KMCS and experimental results as a function of respectively the different substrate temperatures for a $20 \mathrm{~Hz}$ deposition rate, and the different deposition rates for a substrate temperature of $800{ }^{\circ} \mathrm{C}$. The KMCS results show good qualitative agreement with respect to the evolution of pillar density upon changes in synthesis parameters, with a difference of a factor of 100 . This difference is caused by the $E_{s}$ values used in the KMC simulation. For the standard simulation, $\mathrm{E}_{\mathrm{s}}=1.0 \mathrm{eV}$ was used, which generates good results for a low computational cost. Decreasing the value of $E_{s}$ (open squares in Figure 3-5b) allows more hops between consecutive pulses, yielding number density values closer to experiment, but with significantly longer computational times. Extrapolating KMCS number densities for different $E_{s}$ values from Figure 3-5b to the experimental number density of $7.6 \times 10^{-5} \mathrm{~nm}^{-2}$ for a $50 \mathrm{~Hz}$ deposition rate, an $E_{s}$ value of $0.38 \mathrm{eV}$ is calculated, resulting in an estimated increase of computational time for the KMC simulation of two orders of magnitude.
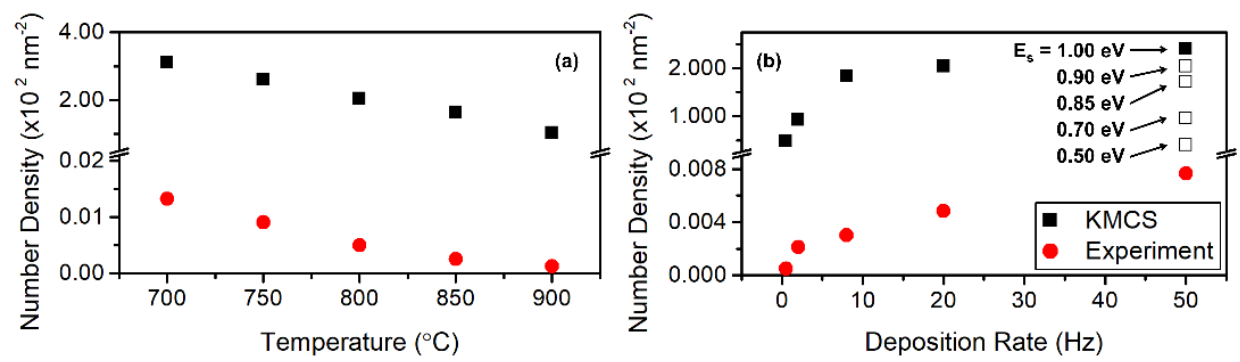

Figure 3-5. Number density comparison between KMCS (ם) and experimental results (O) as a function of (a) different substrate temperatures for a $20 \mathrm{~Hz}$ deposition rate, and (b) different deposition rates for a substrate temperature of $800^{\circ} \mathrm{C}$. White squares $(\square)$ in (b) represent different $\mathrm{E}_{\mathrm{s}}$ values used during simulations.

\subsection{Conclusion}

In this chapter, the successful use of KMCS to model the growth of lithium-based vertically aligned nanocomposite films was demonstrated, consisting of a promising LMO-LLTO cathode-electrolyte combination towards 3D solid-state batteries. For the 
algorithm, a more realistic and low computational cost model was applied, where a minimal number of restrictions was used for diffusion and for which the interaction energies were based on experimental RHEED measurements. The simulations, both of the individual components and of the nanocomposite VAN structures showed good agreement with the films obtained experimentally and followed the trends for deposition rate and temperature dependencies predicted by physical models. The simulated and experimental results show that growing at low deposition frequencies does not generate vertically aligned nanostructures, which is expected to be related to the islands formed during the initial growth stage. On the other hand, higher deposition frequencies yield comparable diffusion lengths, resulting in well defined, but smaller, vertical nanopillars. The inverse effect is expected for variations in deposition temperature, where higher temperatures cause the formation of larger epiphyte grains. Quantitative analysis shows good agreement with respect to the evolution of nanopillar density upon changes in synthesis parameters, with a difference factor explained by the choice of energies used in the KMCS modeling to minimize the computational time. The results demonstrate that KMCS modeling can be successfully applied to predict the nanopillar-matrix formation in lithium-based VAN films. However, to achieve optimal electrochemical functionality in the created VAN structures for energy storage, the specific crystal structure and stoichiometry must be taken into consideration for the individual cathode and electrolyte materials, as well as for the interface formed between them. In Chapter 4, a deeper analysis of the influence of different growth conditions over the structure and electrochemistry of the VAN films was carried out.

\section{Bibliography}

[1] Thostenson, E. T., Li, C., Chou, T. W., Nanocomposites in context. Compos. Sci. Technol. 2005, 65, 491-516.

[2] Palmero, P., Structural Ceramic Nanocomposites: A Review of Properties and Powders' Synthesis Methods. Nanomaterials 2015, 5, 656-696.

[3] Stuart, M. A. C., Huck, W. T. S., Genzer, J., Müller, M., Ober, C., Stamm, M., Sukhorukov, G. B., Szleifer, I., Tsukruk, V. V, Urban, M., Winnik, F., Zauscher, S., Luzinov, I., Minko, S., Emerging applications of stimuli-responsive polymer materials. Nat. Mater. 2010, 9, 101-13.

[4] Zhang, W., Ramesh, R., MacManus-Driscoll, J. L., Wang, H., Multifunctional, selfassembled oxide nanocomposite thin films and devices. MRS Bull. 2015, 40, 736-745.

[5] Huang, J., MacManus-Driscoll, J. L., Wang, H., New epitaxy paradigm in epitaxial selfassembled oxide vertically aligned nanocomposite thin films. J. Mater. Res. 2017, 32, 4054-4066.

[6] Zheng, H., Wang, J., Lofland, S. E., Ma, Z., Mohaddes-Ardabili, L., Zhao, T., Salamanca-Riba, L., Shinde, S. R., Ogale, S. B., Bai, F., Viehland, D., Jia, Y., Schlom, 
D. G., Wuttig, M., Roytburd, A., Ramesh, R., Multiferroic $\mathrm{BaTiO}_{3}-\mathrm{CoFe}_{2} \mathrm{O}_{4}$ nanostructures. Science 2004, 303, 661-663.

[7] Harrington, S. A., Zhai, J., Denev, S., Gopalan, V., Wang, H., Bi, Z., Redfern, S. A. T., Baek, S. H., Bark, C. W., Eom, C. B., Jia, Q., Vickers, M. E., MacManus-Driscoll, J. L., Thick lead-free ferroelectric films with high Curie temperatures through nanocompositeinduced strain. Nat. Nanotechnol. 2011, 6, 491-495.

[8] Wang, Z., Li, Y., Viswan, R., Hu, B., Harris, V. G., Li, J., Viehland, D., Engineered magnetic shape anisotropy in $\mathrm{BiFeO}_{3}-\mathrm{CoFe}_{2} \mathrm{O}_{4}$ self-assembled thin films. ACS Nano $2013,7,3447-3456$.

[9] Chen, A., Bi, Z., Tsai, C. F., Lee, J., Su, Q., Zhang, X., Jia, Q., MacManus-Driscoll, J. L., Wang, H., Tunable low-field magnetoresistance in $\left(\mathrm{La}_{0.7} \mathrm{Sr}_{0.3} \mathrm{MnO}_{3}\right)_{0.5}:(\mathrm{ZnO})_{0.5}$ selfassembled vertically aligned nanocomposite thin films. Adv. Funct. Mater. 2011, 21, $2423-2429$.

[10] Hsieh, Y. H., Liou, J. M., Huang, B. C., Liang, C. W., He, Q., Zhan, Q., Chiu, Y. P., Chen, Y. C., Chu, Y. H., Local conduction at the $\mathrm{BiFeO}_{3}-\mathrm{CoFe}_{2} \mathrm{O}_{4}$ tubular oxide interface. Adv. Mater. 2012, 24, 4564-4568.

[11] Lee, O., Harrington, S. A., Kursumovic, A., Defay, E., Wang, H., Bi, Z., Tsai, C. F., Yan, L., Jia, Q., MacManus-Driscoll, J. L., Extremely high tunability and low loss in nanoscaffold ferroelectric films. Nano Lett. 2012, 12, 4311-4317.

[12] MacManus-Driscoll, J. L., Suwardi, A., Wang, H., Composite epitaxial thin films: A new platform for tuning, probing, and exploiting mesoscale oxides. MRS Bull. 2015, 40, $933-$ 942.

[13] Mouton, I., Talbot, E., Pareige, C., Lardé, R., Blavette, D., The early stage of formation of self-organized nanocolumns in thin films: Monte Carlo simulations versus atomicscale observations in Ge-Mn. J. Appl. Phys. 2014, 115, 053515.

[14] Ichino, Y., Yoshida, Y., Miura, S., Three-dimensional Monte Carlo simulation of nanorod self-organization in $\mathrm{REBa}_{2} \mathrm{Cu}_{3} \mathrm{O}_{\mathrm{y}}$ thin films grown by vapor phase epitaxy. $\mathrm{Jpn}$. J. Appl. Phys. 2017, 56, 015601.

[15] Ichino, Y., Tsuruta, A., Miura, S., Yoshida, Y., Yoshizumi, M., Izumi, T., Determinant for self-organization of $\mathrm{BaMO}_{3}$ nanorods included in vapor-phase-grown $\mathrm{REBa}_{2} \mathrm{Cu}_{3} \mathrm{O}_{\mathrm{y}}$ films. IEEE Trans. Appl. Supercond. 2015, 25, 6995944.

[16] Hennes, M., Schuler, V., Weng, X., Buchwald, J., Demaille, D., Zheng, Y., Vidal, F., Growth of vertically aligned nanowires in metal-oxide nanocomposites: Kinetic MonteCarlo modeling: Versus experiments. Nanoscale 2018, 10, 7666-7675.

[17] Otomo, J., Kurokawa, R., Takahashi, H., Nagamoto, H., Kinetic process of phase separation in $\mathrm{Co}-\mathrm{SiO}_{2}$ thin films and preparation of mesoporous $\mathrm{SiO}_{2}$ thin films with mesopore channels aligned perpendicularly to substrate surfaces. Vacuum 2007, 81, $1003-1011$.

[18] Long, J. W., Dunn, B., Rolison, D. R., White, H. S., Three-dimensional battery architectures. Chem. Rev. 2004, 104, 4463-4492.

[19] Oudenhoven, J. F. M., Baggetto, L., Notten, P. H. L., All-solid-state lithium-ion microbatteries: A review of various three-dimensional concepts. Adv. Energy Mater. $2011,1,10-33$. 
[20] M.Thackeray, M., Manganese oxides for lithium batteries. Prog. Solid State Chem. 1997, $25,1-71$.

[21] Stramare, S., Thangadurai, V., Weppner, W., Lithium Lanthanum Titanates: A Review. Chem. Mater. 2003, 15, 3974-3990.

[22] Zheng, H., Straub, F., Zhan, Q., Yang, P. L., Hsieh, W. K., Zavaliche, F., Chu, Y. H., Dahmen, U., Ramesh, R., Self-assembled growth of $\mathrm{BiFeO}_{3}-\mathrm{CoFe}_{2} \mathrm{O}_{4}$ nanostructures. Adv. Mater. 2006, 18, 2747-2752.

[23] Herger, R., Willmott, P. R., Bunk, O., Schlepütz, C. M., Patterson, B. D., Delley, B., Shneerson, V. L., Lyman, P. F., Saldin, D. K., Surface structure of $\mathrm{SrTiO}_{3}(001)$. Phys. Rev. B - Condens. Matter Mater. Phys. 2007, 76, 195435.

[24] Hendriks, R., Cunha, D. M., Singh, D. P., Huijben, M., Enhanced Lithium Transport by Control of Crystal Orientation in Spinel $\mathrm{LiMn}_{2} \mathrm{O}_{4}$ Thin Film Cathodes. ACS Appl. Energy Mater. 2018, 1, 7046-7051.

[25] Ohta, H., Mizoguchi, T., Aoki, N., Yamamoto, T., Sabarudin, A., Umemura, T., Lithium-ion conducting $\mathrm{La}_{2} / 3 \times \mathrm{Li}_{3 \times} \mathrm{TiO}_{3}$ solid electrolyte thin films with stepped and terraced surfaces. Appl. Phys. Lett. 2012, 100, 173107.

[26] Metropolis, N., The beginning of the Monte Carlo Method. Los Alamos Sci. 1987, 125 130.

[27] Beeler Jr., J. R., Displacement Spikes in Cubic Metals. I. a-Iron, Copper, and Tungsten. Phys. Rev. 1966, 150, 470-487.

[28] Gordon, R., Adsorption isotherms of lattice gases by computer simulation. J. Chem. Phys. 1968, 48, 1408-1409.

[29] Gilmer, G. H., Growth on imperfect crystal faces. I. Monte-Carlo growth rates. J. Cryst. Growth 1976, 36, 15-28.

[30] Rikvold, P. A., Simulations of a stochastic model for cluster growth on a square lattice. Phys. Rev. A 1982, 26, 647-650.

[31] Voter, A. F., Classically exact overlayer dynamics: Diffusion of rhodium clusters on Rh(100). Phys. Rev. B 1986, 34, 6819-6829.

[32] Bortz, A. B., Kalos, M. H., Lebowitz, J. L., A new algorithm for Monte Carlo Simulation of Ising Spin Systems. J. Comput. Phys. 1975, 17, 10-18.

[33] Norris, J. R., Markov Chains. Cambridge University Press, Cambridge 1997.

[34] Feibelman, P. J., Diffusion path for an $\mathrm{Al}$ adatom on Al(001). Phys. Rev. Lett. 1990, 65, 729-732.

[35] Liu, C.-L., Adams, J. B., Structure and diffusion of clusters on Ni surfaces. Surf. Sci. 1992, 268, 73-86.

[36] Wang, R., Fichthorn, K. A., An Investigation of Adsorption-Induced Smoothing Mechanisms in Pt/Pt(111) Homoepitaxy. Mol. Simul. 1993, 11, 105-120.

[37] Hamilton, J. C., Daw, M. S., Foiles, S. M., Dislocation mechanism for island diffusion on fcc (111) surfaces. Phys. Rev. Lett. 1995, 74, 2760-2763. 
[38] Uberuaga, B. P., Smith, R., Cleave, A. R., Montalenti, F., Henkelman, G., Grimes, R. W., Voter, A. F., Sickafus, K. E., Structure and Mobility of Defects Formed from Collision Cascades in MgO. Phys. Rev. Lett. 2004, 92, 19-22.

[39] Voter, A. F., in: Sickafus, K. E., Kotomin, E. A., Uberuaga, B. P. (Eds.), Radiation Effects in Solids. Springer Netherlands, Dordrecht 2007, pp. 1-23.

[40] Ratsch, C., Zangwill, A., Smilauer, P., Vvedensky, D. D., Saturation and scaling of epitaxial island densities. Phys. Rev. Lett. 1994, 72, 3194-3197.

[41] Lam, P. M., Liu, S. J., Woo, C. H., Monte Carlo simulation of pulsed laser deposition. Phys. Rev. B - Condens. Matter Mater. Phys. 2002, 66, 454081-454086.

[42] Ehrlich, G., Atomic displacements in one- and two-dimensional diffusion. J. Chem. Phys. 1966, 44, 1050-1055.

[43] Schwoebel, R. L., Shipsey, E. J., Step motion on crystal surfaces. J. Appl. Phys. 1966, 37, 3682-3686.

[44] Meyer, J. A., Vrijmoeth, J., van der Vegt, H. A., Vlieg, E., Behm, R. J., Importance of the additional step-edge barrier in determining film morphology during epitaxial growth. Phys. Rev. B 1995, 51, 790-793.

[45] Maksym, P. A., Fast Monte Carlo simulation of MBE growth. Semicond. Sci. Technol. 1988, 3, 594-596.

[46] Rijnders, G., Blank, D. H. A., Choi, J., Eom, C.-B., Enhanced surface diffusion through termination conversion during epitaxial $\mathrm{SrRuO}_{3}$ growth. Appl. Phys. Lett. 2004, 84, 505507.

[47] Zheng, S., Zhu, W., Gao, Y. F., Stocks, G. M., Zhang, Z., Kinetic Monte Carlo simulations of nanocolumn formation in two-component epitaxial growth. Appl. Phys. Lett. 2010, 96, 071913.

[48] Walter, J. C., Barkema, G. T., An introduction to Monte Carlo methods. Phys. A Stat. Mech. its Appl. 2015, 418, 78-87. 


\title{
Chapter 4
}

\section{Li-based Vertically Aligned Nanocomposites}

\begin{abstract}
The complex oxides class exhibits an amazing range of functional properties far exceeding those of the semiconductors that are abundant in the current electronics. Exploiting vertically aligned nanocomposite (VAN) films to create new device architectures and multifunctionalities is a concept that has been explored in the last 15 years. Such structures have powerful advantages over conventional multilayer structures, due to the possibility of self-assembly, greater interfacial area, and strain tunability to large thicknesses. A careful tuning of thermodynamic parameters (e.g. temperature, composition, and bond energies) is required to control the overall energy of the system to create the desired structures, while control of the kinetics is required to tune the length scales. To design VAN structures, it is necessary to understand the different mechanisms of composite nanostructure formation in thin films, and the compositional, structural, and processing factors that influence these. The influence of deposition temperature and frequency on the morphology evolution of the $\mathrm{LiMn}_{2} \mathrm{O}_{4} /(\mathrm{Li}, \mathrm{La}) \mathrm{TiO}_{3}$ system was investigated previously in chapter 3 . In this chapter, a study of the influence of these parameters, along with the substrate crystal orientation, on the structure and composition of the VANs will be carried out. The possibility of adjusting the diffusivity of the arriving species on the substrate surface by means of buffer layers, and the implications of increasing the film thickness, will be analyzed. A step towards the integration of VANs with Silicon technology is given via buffered crystalline STO layers, and the impact on the structure is assessed. Finally, electrochemical analysis of the Lithium-based VANs is performed, and the effect of structure and composition on the VAN functionality is explored.
\end{abstract}




\subsection{Introduction}

The complex oxides class of materials exhibits an amazing range of functional properties far exceeding those of the semiconductors that are abundant in the current electronics, e.g. high-temperature superconductivity, room-temperature magnetic semiconductivity, colossal magnetoresistance, ferroelectricity, and multiferroicity.[1-4] While there has been limited work on self-assembly of inorganic structures, over the last 30 years there has been much work on organic systems. There is every reason to believe that similar developments in the inorganic area will lead to a range of new interfacecoupled thin-film functionalities.

Conventional interfacial structures are usually in the form of heteroepitaxial multilayers. Utilizing vertically aligned nanocomposite (VAN) films to create new, selfassembled device architectures and multifunctionalities is a concept that has been explored in the last 15 years.[5,6] Such structures have powerful advantages over conventional multilayer structures, due to the possibility of self-assembly, greater interfacial area, and strain tunability to large thicknesses. Most device materials technologies rely on planar films grown on a single crystal substrate surface, which epitaxially strains the thin films up to a critical layer thickness, on the order of a few nanometers, depending on the amount of the mismatch between the involved crystal structures. Above the critical thickness, the strain is relieved, in the form of misfit dislocations and, commonly above tens of nanometers, significant strain relaxation occurs.[7] Self-assembled VAN structures have the great advantage over planar films as the strain is controlled by the numerous vertical interfaces and not the single horizontal interface with the substrate.

A careful tuning of thermodynamic parameters (e.g. temperature, composition, and bond energies) is required to control the overall energy of the system to create the desired structures, while control of the kinetics is required to tune the length scales. To design VAN structures, it is required to understand the different mechanisms of composite nanostructure formation in films, and the compositional, structural, and processing factors that influence these. The influence of temperature and frequency on the morphology evolution of the $\mathrm{LiMn}_{2} \mathrm{O}_{4} /(\mathrm{Li}, \mathrm{La}) \mathrm{TiO}_{3}$ (LMO/LLTO) system was investigated previously in chapter 3 . In this chapter, a study of the influence of these parameters, along with the substrate crystal orientation, on the structure and composition of the VANs will be carried out. 


\subsubsection{Conditions and Mechanisms of VAN Film Growth}

Oxide VAN film structures are composed of two intermixed materials, grown typically by pulsed laser deposition on a single crystal substrate from a mixed target. The most thermodynamically stable phase or phases (considering epitaxial stabilization) will grow on the substrate, which are not necessarily the same phases present in the target.

Consider the case of growing an oxide film with a mixture of phases of $\mathrm{AO}+\mathrm{BO}$, where A could be a single cation or combination of cations, and B could be a single cation or combination of cations that might include one or more of the cations in A. A composite target precursor material containing all the chemical constituents, with different ratios between $\mathrm{AO}$ and $\mathrm{BO}$, would typically be used to grow a VAN film. There are four basic conditions that give rise to the whole range of possible film compositions and microstructures, including VAN structures, [8] namely:

1. Immiscibility, where no solid solution exists between $\mathrm{AO}$ and BO. From equilibrium thermodynamics the two phases will form by nucleation and growth;

2. Miscibility of $\mathrm{A}, \mathrm{B}$, and $\mathrm{O}$, where a single phase of $(\mathrm{A}, \mathrm{B}) \mathrm{O}$ will form by nucleation and growth;

3. Miscibility at high temperatures with clustering at lower temperatures. Depending on the overall composition of the starting mixture, two phases will form by either nucleation and growth, or spinodal growth;

4. Partial miscibility with coherent precipitation of a low symmetry phase in a matrix of a higher symmetry phase.

The three basic growth mechanisms derived from the four mechanisms of phase formation are nucleation and growth, spinodal decomposition and pseudo-spinodal decomposition. While the different growth mechanisms will be discussed in the following section, the discussion will be focused on nucleation and growth as this behavior is present during the LMO/ LLTO VAN formation, as described in the previous chapter.

\section{Nucleation and Growth}

For a system with complete miscibility, growth of a single-phase film occurs on the substrate, and the orientation of such a film will be dictated by the epitaxial growth relation with the substrate. For VAN thin film growth, two phases need to be produced, consequently they must be immiscible. Two phases form in the film by nucleation and growth with size, orientation, and phase distribution that depend on epitaxial constraints. The microstructures formed are determined by the ratio A:B, their crystallographic 
relations, the surface energies between the two phases (and between each phase and the substrate), and the growth kinetics.

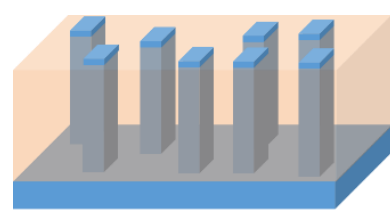

(a)

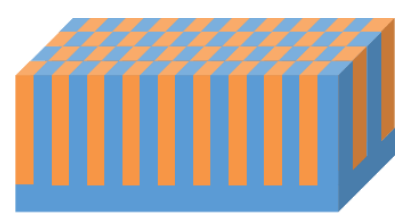

(b)

Figure 4-1. Illustration of vertically aligned nanocomposites obtained by (a) high A:B ratio nucleation and growth and (b) spinodal decomposition.

In the high $\mathrm{A}: \mathrm{B}$ ratio regime (30:70 range), illustrated in Figure 4-1a, the morphology of the microstructure depends on the different interfacial energies of $\mathrm{AO}$ and $\mathrm{BO}$ with the given substrate surface. A good example is the well-studied mixture of the two immiscible phases, the perovskite $\mathrm{BiFeO}_{3}(\mathrm{BFO})$ and the spinel $\mathrm{CoFe}_{2} \mathrm{O}_{4}(\mathrm{CFO})$. Growth on single crystal (001) $\mathrm{SrTiO}_{3}$ (STO) has shown that (001) BFO completely wets the STO surface as the interfacial energy between these two crystallographically matched perovskite surfaces is low.[9,10] In contrast, the (111) plane is the lowest surface energy plane for the CFO, so it grows on STO(001) with the (001) plane parallel to it but with the four (111) faces growing out of the plane of the substrate to form pyramidal crystallites, as also demonstrated in chapter 2 for the spinel $\mathrm{LiMn}_{2} \mathrm{O}_{4}$ and $\mathrm{Li}_{4} \mathrm{Ti}_{5} \mathrm{O}_{12}$ thin films. The resulting microstructure, therefore, displays surface pyramidal crystallites of $\mathrm{CFO}$ embedded in planar BFO. On a STO (111) surface, this behavior is reversed as the CFO (111) is more favorable to grow, completely wetting the substrate surface, with BFO crystallites.[9] In all cases of substrate orientation, the crystallite domains are typically around $100 \mathrm{~nm}$ in width. With increasing ratio of BFO to CFO, the growth mode of the films remain the same but with different sizes and/or densities of the embedded crystallites.

Similar structures can be obtained also for the VAN system with $\mathrm{BaTiO}_{3}$ (BTO) and $\mathrm{CFO}$, except that the CFO islands form into more cylindrical pillars rather than pyramidal.[6,11,12] The micro-faceting present in the cylindrical pillars is on a much finer scale and is commensurate with the pillar diameter, which is also smaller, typically $<50 \mathrm{~nm}$. This difference in pillar diameter is given by different kinetics on the surface for BFO and BTO. Although both materials have the perovskite structure, as the melting point of $\mathrm{BFO}$ is $\sim 500{ }^{\circ} \mathrm{C}$ lower than BTO, for the same growth temperature the mobility of species on the film surface is greater for BFO, resulting in larger pillar diameters. [8] Although no clear evidence of spatial ordering of phases by standard nucleation and growth of a two-phase mixture have been reported, partial ordering of structures can be 
produced as a result of long range elastic interaction,[13] and ordered BFO-CFO VANs could be obtained by nucleation-induced self-assembly process.[14]

The most widely studied system in the low ratio regime (10:90 range) is $\mathrm{YBa}_{2} \mathrm{Cu}_{3} \mathrm{O}_{7}$ (YBCO) containing immiscible second phase additions. If $\mathrm{YBCO}(\mathrm{BO})$ is the matrix and $\mathrm{Gd}_{3} \mathrm{TaO}_{7}(\mathrm{AO})$ the minor phase, for example, then either randomly dispersed, very small $(<5 \mathrm{~nm})$ nanoparticles of $\mathrm{Gd}_{3} \mathrm{TaO}_{7}$ embedded in $\mathrm{YBCO}[15]$ having incoherent or semicoherent interfaces,[16] or self-assembled columnar structures of nanoparticles are observed, similar to self-assembled semiconductor quantum dots.[13,17,18] Which of these structures will form is a result of growth kinetics, as slow growth rates and high growth temperatures allow sufficient time for AO to migrate over the BO surface. It is more favorable for the $\mathrm{AO}$ phase to nucleate on a strained region of the $\mathrm{BO}$ surface, where the $\mathrm{AO}$ lies beneath it, producing a thin columnar structure of $\mathrm{AO}$ in a $\mathrm{BO}$ matrix.[19]

\section{Spinodal and pseudo-spinodal decomposition}

Spinodal decomposition, illustrated in Figure 4-1b, requires a positive enthalpy of mixing with a tendency toward clustering at low temperatures. Spinodal fluctuations in composition create coexisting regions with slightly different lattice parameters, leading to local strains in the decomposing crystal. Minimization of strain energy leads to spatial organizations of the composition fluctuations that are dictated by the symmetry of the elastic properties. The coexisting regions coarsen over time from around $5 \mathrm{~nm}$ to around $50 \mathrm{~nm}$, and they become highly ordered.[20] Spinodal decomposition has received limited attention in oxide thin films, which is surprising since oxides typically exhibit wide miscibility of different cations, e.g. $\mathrm{RE}_{1-\mathrm{x}} \mathrm{AE}_{\mathrm{x}} \mathrm{MnO}_{3}$, where $\mathrm{RE}=$ rare earth and $\mathrm{AE}=$ alkaline earth, and that such mixing is a prerequisite for spinodal decomposition. While many efforts to study spinodal decomposition are focused on bulk materials, [21-23] films from $\mathrm{Zn}(\mathrm{Mn}, \mathrm{Ga})_{2} \mathrm{O}_{4}$ have also been grown,[24] where regularly spaced checkerboard domains of alternating orthorhombic (Mn-rich) and tetragonal (Mn-poor) regions of $9 \mathrm{~nm}$ size were observed. This checkerboard structure forms to reduce the volume strain energy associated with the lattice misfit between the two different domain compositions and it also reduces the formation of strain relieving misfit dislocations.[25]

Pseudo-spinodal growth contains elements of both spinodal decomposition and nucleation and growth and was first observed in metallic alloys 40 years ago.[26] It is particularly important in the Co-Pt ferromagnetic memory phases.[27] Compared to standard spinodal decomposition, pseudo-spinodal growth gives the possibility of a checkerboard structure in a wider region of compositional space since it does not rely on the presence of complete miscibility at high temperatures with a miscibility gap opening up at lower temperatures. Instead, it occurs by decomposition of an initially random 
mixture of cations into coherent precipitates of a low symmetry phase embedded in a matrix of high symmetry phase, e.g. growth of tetragonal precipitates in a cubic matrix.

\subsection{Experimental}

The films studied in this chapter were grown from a sintered $67 \% \mathrm{La}_{0.5} \mathrm{Li}_{0.5} \mathrm{TiO}_{3}+33 \%$ $\mathrm{LiMn}_{2} \mathrm{O}_{4}(30 \mathrm{wt} \%$ excess $\mathrm{Li}$ ) target, with a target-substrate distance of $5 \mathrm{~cm}$, at an oxygen pressure of 0.2 mbar within the temperature range $700-900{ }^{\circ} \mathrm{C}$. A $\mathrm{KrF}$ excimer laser was used operating at $248 \mathrm{~nm}, 8-50 \mathrm{~Hz}$ and a laser energy fluence of $2.3 \mathrm{~J} . \mathrm{cm}^{-2}$ resulting in a growth rate of $\sim 0.15 \AA$ Apulse. The films were grown using 7200 pulses, except if mentioned otherwise. The STO substrates $(a=3.905 \AA$ )[28] were pre-annealed in a tube-oven at $950{ }^{\circ} \mathrm{C}$ for $90 \mathrm{~min}$ in an oxygen flow of $150 \mathrm{~L} / \mathrm{h}$. After deposition, the films were cooled down to room temperature in an oxygen pressure of $0.2 \mathrm{mbar}$ at a rate of 10 ${ }^{\circ} \mathrm{C} / \mathrm{min}$. The structural parameters of the films were analyzed using X-ray diffraction (XRD) measurements, performed on a PANalytical X'Pert PRO diffractometer, with a PIXcel1D detector and a Cu source $(\lambda=1.5406 \AA)$. The surface morphology was studied using Tapping-mode atomic force microscopy (AFM), carried out in air on a Bruker ICON Dimension Microscope. The size and distribution of the structures measured by AFM were obtained with Gwyddion software. Cross-sectional images and qualitative compositional analysis were done with a Zeiss Merlin high-resolution scanning electron microscopy (HRSEM).

\subsection{Target composition}

Pulsed laser deposition (PLD) is a physical vapor deposition (PVD) technique based on the evaporation of material through a highly energetic laser beam, which, focused by a lens, shows a high enough energy density for ablation of the source material. The vaporized material forms a plasma plume, propagating through the chamber and depositing onto the substrate. One of the advantages of PLD over other deposition methods is the capability for stoichiometric transfer of material from target to substrate,[29] where the exact chemical composition of a complex material can be reproduced in the film if the focused laser energy density is chosen properly.[30] Special attention has to be given for material's composition which includes light elements (e.g. $\mathrm{Li}, \mathrm{Na}, \mathrm{K}, \mathrm{Be}, \mathrm{Mg}$ ), as the target composition have to be compensated for losses due the materials' desorption from the surface. 


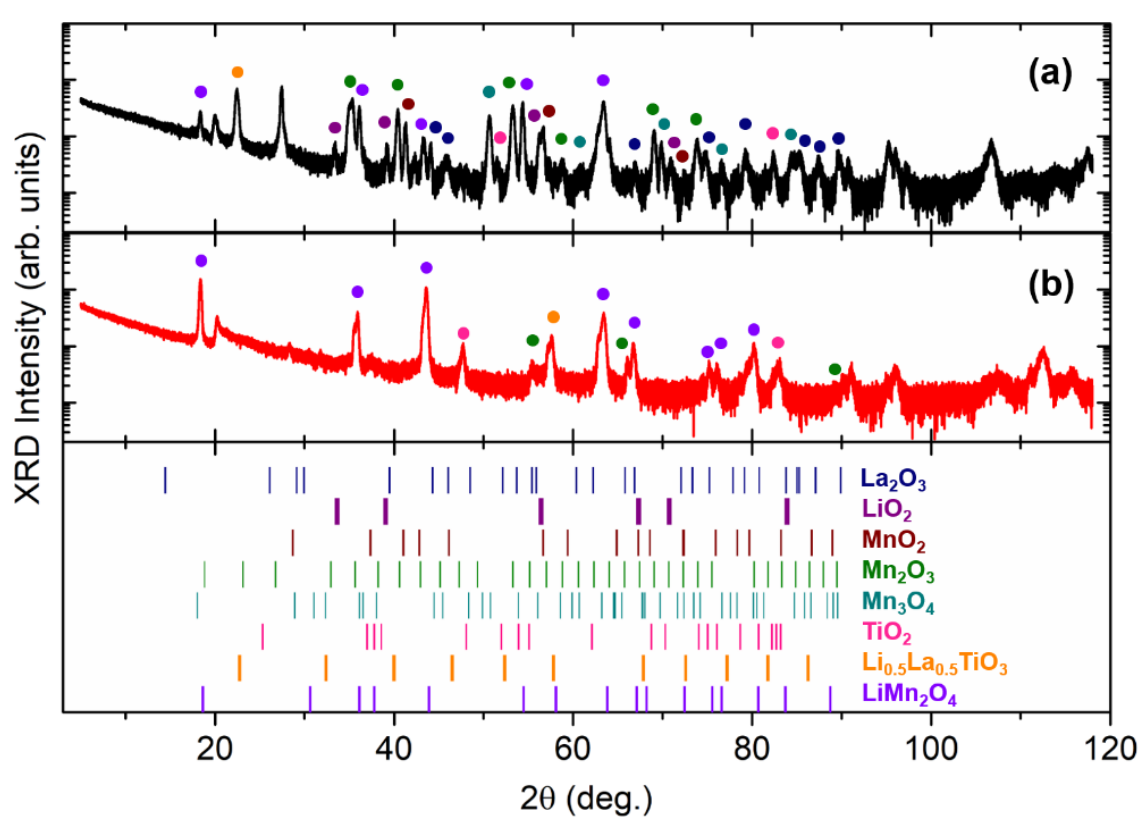

Figure 4-2. XRD pattern showing the stoichiometry of (a) Target 1 and (b) Target 2. Both targets have the same composition, but different starting phases. Below are the characteristic XRD peak positions for different polycrystalline oxide compounds containing Li, La, Ti and/or Mn.

The XRD pattern for two targets with exact same composition, that is $67 \%$ $\mathrm{La}_{0.5} \mathrm{Li}_{0.5} \mathrm{TiO}_{3}+33 \% \mathrm{LiMn}_{2} \mathrm{O}_{4}$, is shown in Figure 4-2. Although both targets present the same elemental composition, structural characterization shows that the targets are composed by different crystallographic phases. While Target 1 presents multiple phases, e.g. LMO, LLTO, $\mathrm{La}_{2} \mathrm{O}_{3}, \mathrm{TiO}_{2}, \mathrm{LiO}_{2}$ and $\mathrm{Mn}_{\mathrm{x}} \mathrm{O}_{\mathrm{y}}$, Target 2 displays mainly $\mathrm{LMO}$ peaks with contributions of LLTO, $\mathrm{TiO}_{2}$ and $\mathrm{Mn}_{2} \mathrm{O}_{3}$, which highlights the difference in complexity between both targets.

The structural analysis of the films deposited with the same growth condition are depicted by the XRD patterns in Figure 4-3a,b. Films were grown at $0.2 \mathrm{mbar} \mathrm{O}_{2}$, substrate temperature of $800{ }^{\circ} \mathrm{C}$, and deposition rate of $20 \mathrm{~Hz}$. A priori, as the materials are ablated and ionized into the plasma plume, different crystallographic phases in the targets should not influence the composition of the deposited films. However, Figure 4-3a,b shows formation of different crystallographic phases. Both films display the peak expected for LMO, but with a considerable shift. The thin film grown from Target 1 (black line) shows the LMO peak at $44.7^{\circ}$ (Li-poor LMO phase), while films grown from Target 2 (red line) show the LMO peak at $43.4^{\circ}$ (Li-rich LMO phase), compared to the expected $43.9^{\circ}$ for LMO. Possible explanations for the peak shift will be explored in the following sections. The LLTO peaks are only present on the film obtained from Target 2 , although, according to the Target 1's composition, the phase should be present on its film. 

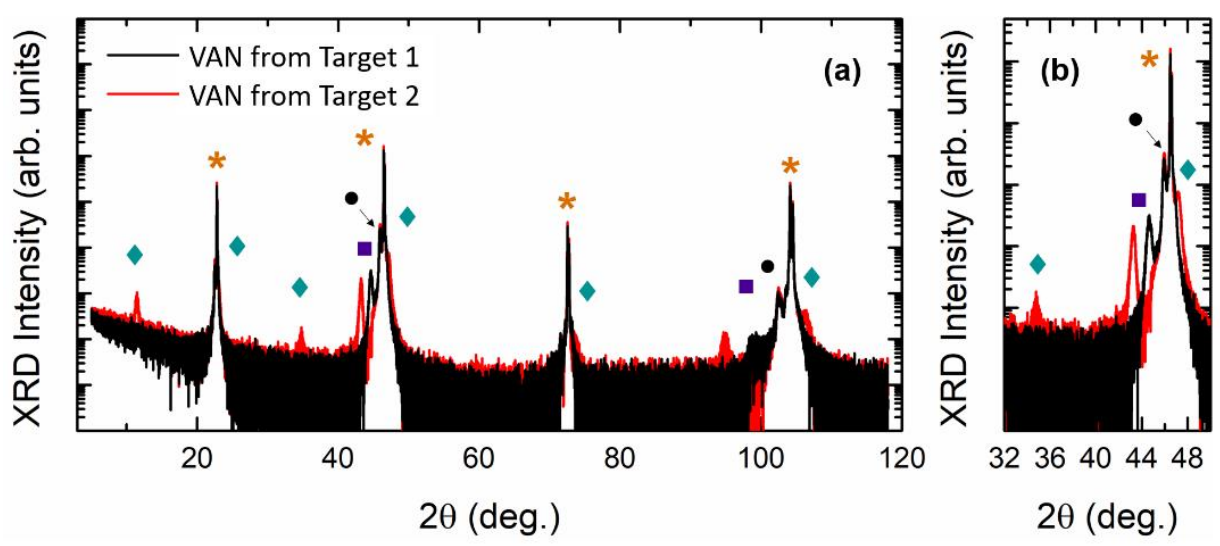

(c)

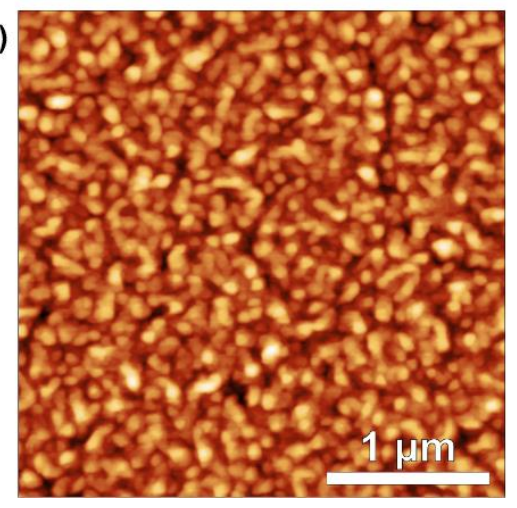

(d)

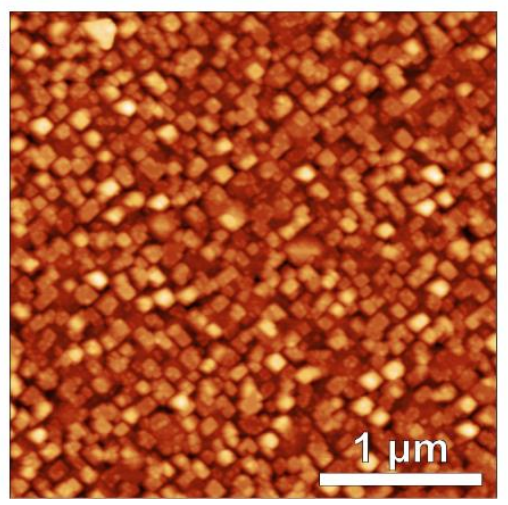

Figure 4-3. (a) Full XRD pattern of the VAN films grown with the same conditions for Target 1 (black) and 2 (red), in which LLTO peaks are shown by $\bullet$, LMO by $\boldsymbol{\square}$, SRO by , and the STO substrate peaks by *. (b) XRD pattern highlighting the peaks between $32^{\circ}-50^{\circ}$. AFM images of the VAN films grown with the same conditions for (c) Target 1 and (d) Target 2.

A difference in morphology is also observed for the films obtained, as displayed by the AFM images in Figure 4-3c,d. While Target 2 yields the expected VAN structure, with square-like features embedded into a matrix, the film obtained from Target 1 shows a grainy surface, with no specific features distinguishable.

Different parameters can influence the interaction of the laser beam with the target material, like the absorption coefficient and reflectivity of the target material, the pulse duration, wavelength and energy of the laser beam. Different phases present in the target (e.g. Target 1), show variation in its absorption properties leading to a less uniform ablation, resulting in different composition and kinetics of the plasma plume. Since the quality of the thin film depends on the absorption/desorption [31] and diffusivity [32,33] of the particles on the surface, for the growth of VANs, it is important to take into account the phases that make up the target and not only its composition, as more components 
increase the complexity of the ablation and kinetics of the plume. For the rest of the study of Li-based VAN formation, Target 2 was chosen, as it provides the desired phases and morphology.

\subsection{Control of Li-based VAN structure during growth}

To design VAN structures, it is required to understand the different mechanisms of composite nanostructure formation in films, and the compositional, structural, and processing factors that influence these. The composition, structure and morphology of Licontaining VAN structures are studied in this section, where a series of thin films were deposited and analyzed for different growth temperatures, depositions rates, substrate crystal orientations and terminations.

\subsubsection{Temperature}

As discussed in chapter 2, the LMO cathode thin films are grown at $600{ }^{\circ} \mathrm{C}$ because at higher temperatures Li desorbs from the surface and only $\mathrm{Mn}_{\mathrm{x}} \mathrm{O}_{\mathrm{y}}$ phases are obtained,[34] whereas the LLTO phase displays its highest crystallinity and performance at $900{ }^{\circ} \mathrm{C}$. Although, due to the strong coupling at the vertical interfaces, VANs allow phases to be stabilized in conditions that would not be possible otherwise. It is expected that at higher temperatures the LLTO matrix would be the most crystalline while Li evaporates from the structure and $\mathrm{Mn}_{\mathrm{x}} \mathrm{O}_{\mathrm{y}}$ phases become predominant in the pillars. For the analysis of the influence of growth temperature on the composition, structure and morphology of the desired VANs, a series of thin films were grown on $\mathrm{Nb}: \mathrm{STO}(100)$ substrate, at an oxygen pressure of 0.2 mbar, deposition rate of $8 \mathrm{~Hz}$, within the temperature range $600-900{ }^{\circ} \mathrm{C}$.

For the discussion on the formation of crystalline VAN thin films temperatures lower than $700{ }^{\circ} \mathrm{C}$ were not used as it was not possible to obtain phase separation and the formation of crystallographic phases, as presented by Figure 4-4. A $50 \mathrm{~nm} \mathrm{SrRuO}_{3}$ (SRO) layer was deposited as an intermediate layer to enhance the electrical transport between the $\mathrm{LiMn}_{2} \mathrm{O}_{4}$ cathode pillars in the VAN structure and the conducting $\mathrm{Nb}: \mathrm{STO}$ substrate.[34,35] 

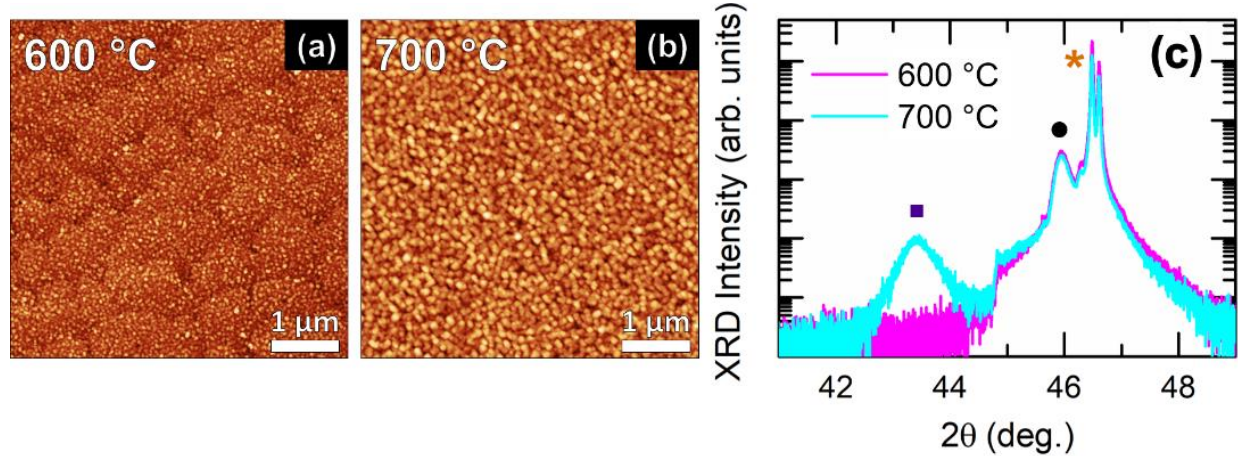

Figure 4-4. AFM images for VAN structures grown at (a) $600{ }^{\circ} \mathrm{C}$ and (b) $700{ }^{\circ} \mathrm{C}$. (c) XRD pattern highlighting the peaks between $40^{\circ}-50^{\circ}$, comparing the crystallographic properties for VAN films grown at $600{ }^{\circ} \mathrm{C}$ and $700{ }^{\circ} \mathrm{C}$.
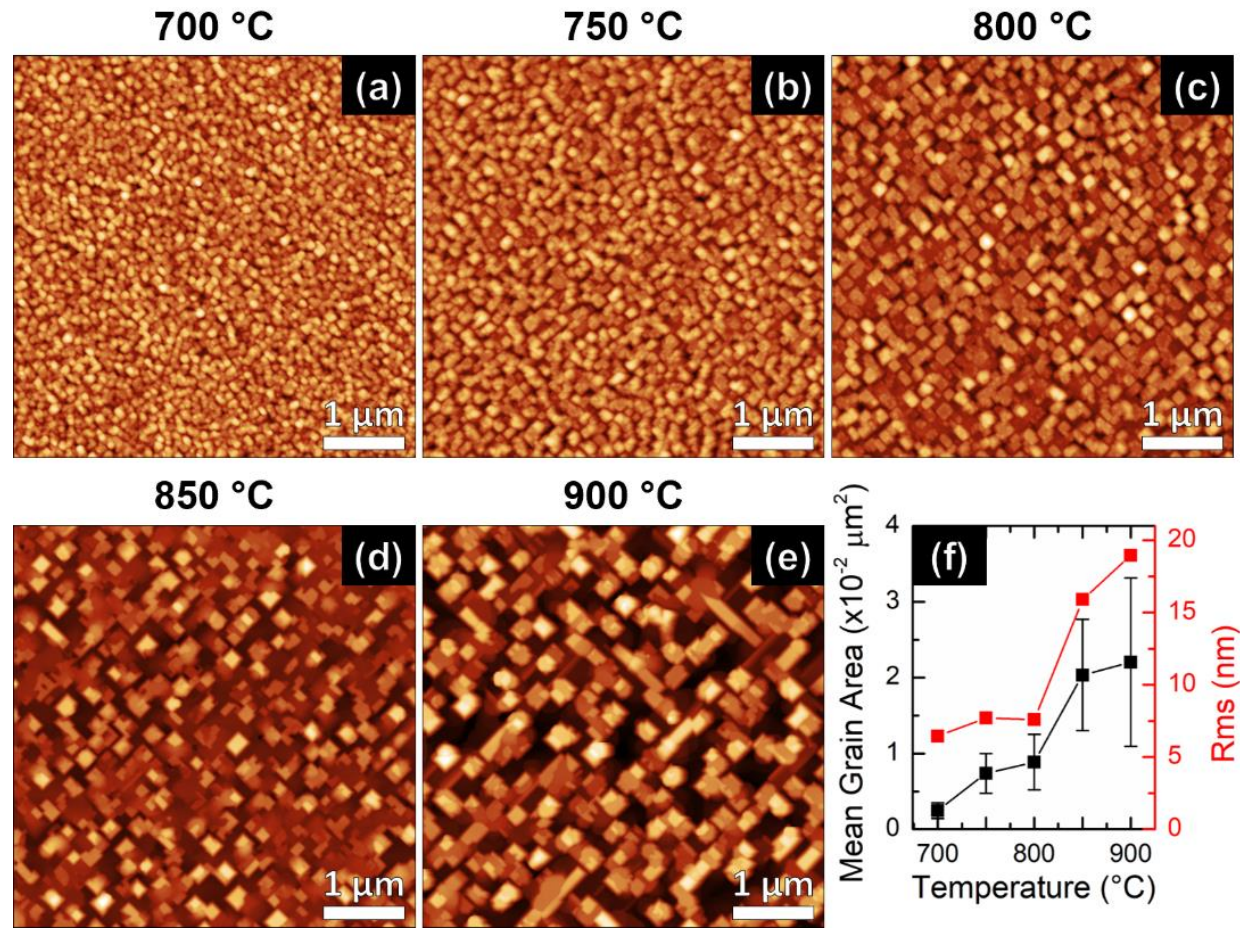

Figure 4-5. (a-e) AFM images for VAN structures grown at different temperatures. (f) Mean grain area and Roughness RMS obtained from the AFM images. 
The AFM images given by Figure 4-5a-d shows the VAN morphology obtained for different growth temperatures. Higher growth temperatures reduce the activation energy for hopping of the adatoms on the surface, increasing the mean diffusion length yielding bigger (but less uniform) grains, $[9,36]$ as presented by the mean grain area dependence on temperature shown in Figure 4-5f, in accordance with the kinetic model presented in chapter 3 and other studies on the growth and formation of VANs.[9,36] Higher growing temperatures results also in an height increase, due the formation of the pillars, as indicated by the rise of RMS values in Figure 4-5f.

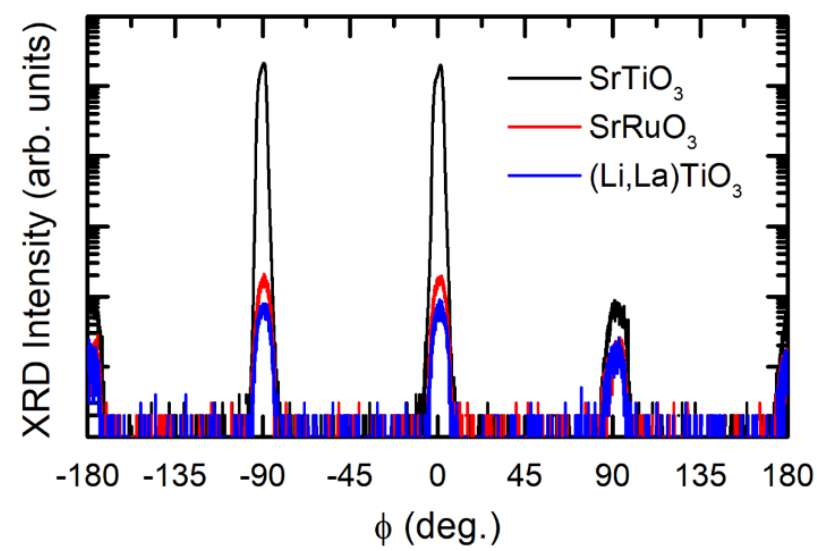

Figure 4-6. In-plane XRD measurements of VAN grown at $750{ }^{\circ} \mathrm{C}$, for the $(013)$ plane. Peaks of the $\mathrm{SrTiO}_{3}, \mathrm{SrRuO}_{3}$ and $(\mathrm{Li}, \mathrm{La}) \mathrm{TiO}_{3}$ are indicated respectively in black, red and blue.

The in-plane crystal orientations of the VAN films were studied by XRD $\varphi$-scans along the (013) direction for STO/SRO/LLTO, see Figure 4-6. The LMO is not permitted at this orientation and, for the (113) orientation, where LMO is allowed, the XRD intensity counts are not enough and therefore, not shown. The separation of the STO peaks in the (100) plane by $90^{\circ}$ is consistent with the perovskite crystal structure, as discussed in chapter 2. For the LLTO(100) and SRO(110) layers the in-plane peaks are at the same angles as the substrate orientation indicating the in-plane alignment of the perovskite LLTO and SRO layer to the underlying STO perovskite substrate.

The deposited LMO, LLTO and SRO layers, exhibit coherent growth in which the outof-plane crystal orientation is aligned with the (001) orientation of the substrate, as shown in Figure 4-7. The VAN thin film show the presence of a highly crystalline epitaxial layer without any impurity phase, suggesting that the PLD deposition process parameters (e.g. temperature, pressure, laser energy density, target composition) were chosen successfully to correct for any loss of volatile lithium during ablation, nucleation or growth. The SRO layer shows the expected lattice parameter of $\sim 3.95 \AA$. The LLTO phase is epitaxially 
strained to the underlying STO substrate as determined by reciprocal space mapping XRD (Figure 4-8 top). The LLTO exhibits an out-of-plane lattice parameter of $\sim 3.85 \AA$, which corresponds to a crystal structure with a volume equal to a relaxed cubic phase of about $\mathrm{Li}_{0.3} \mathrm{La}_{0.57} \mathrm{TiO}_{3}$ with a lattice parameter of $\sim 3.88 \AA$. [37] This indicates a $0.06 \%$ in-plane strain in the LLTO unit cell. The reduction of intensity observed for the LLTO peak for lower temperatures is expected as it requires higher temperatures for enhanced crystallinity.[37,38] The LMO peak shows a shift in lattice parameter of $8.32 \AA$ to 8.45 $\AA$, on the temperature range studied $\left(700{ }^{\circ} \mathrm{C}\right.$ to $\left.900{ }^{\circ} \mathrm{C}\right)$, resulting in unit cell volumes of $576 \AA^{3}$ and $603 \AA^{3}$, respectively. These values of correspond to an increment of at least $2.8 \%$ on the volume of the LMO unit cell, which is related to a high lithiation state $\left(\mathrm{Li}_{\mathrm{x}} \mathrm{Mn}_{2} \mathrm{O}_{4}\right.$, with $\left.\mathrm{x}>1\right)$. [39,40] This shift in peak position also presents an unexpected behavior, as films deposited at higher temperatures contains lower Li concentration due Li evaporation [41] and, thus, would display smaller unit cells.[39,40]
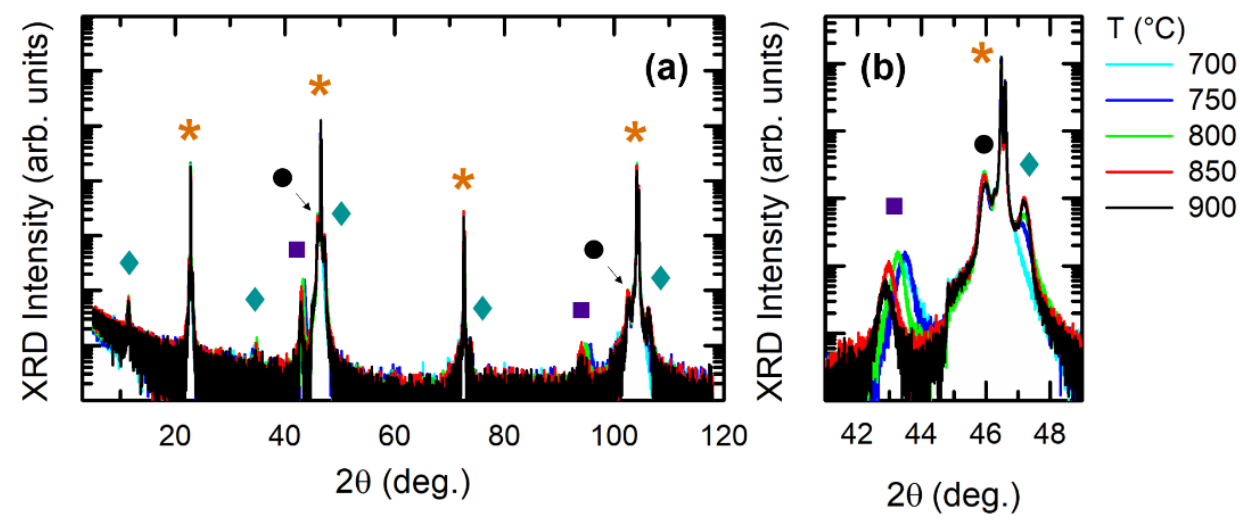

Figure 4-7. (a) Full XRD pattern of the VAN films grown at different temperatures, in which LLTO peaks are shown by $\boldsymbol{\bullet}$, LMO by $\boldsymbol{\square}$, SRO by $\boldsymbol{\bullet}$, and the STO substrate peaks by $\star$. (b) XRD pattern highlighting the peaks between $40^{\circ}-50^{\circ}$.

Due to high temperatures and the availability of Ti in the target, the LMO pillars could be doped with titanium. Cationic substitution, is a known behavior in complex oxide growth, and has been explored for the growth of VANs.[8] The doped cathode material $\mathrm{LiMn}_{2-\mathrm{y}} \mathrm{Ti}_{\mathrm{y}} \mathrm{O}_{4}$ (LMTO) has been investigated as an alternative for $\mathrm{LMO}$, as the $\mathrm{Ti}^{4+}$ cation would bring more stability for the structure, allowing the intercalation of two $\mathrm{Li}^{+}$per unit cell, making use of the $3 \mathrm{~V}$ plateau that would result in Jahn-Teller distortion and structure instability otherwise.[42-44]

Considering the LMTO phase, the shift in peak position could be explained as higher temperatures increase the doping level of $\mathrm{Ti}$ into LMO,[43] expanding the unit cell volume, as observed. The enthalpy of formation for LMTO is also predicted to be $0.8 \mathrm{eV}$ smaller than that of LMO,[43] and depositing all elements at the same time would result 
in the most energetically favorable phase. To understand the structure formed and their relation, reciprocal space mapping (RSM) was performed to obtain the in-plane, as well as out-of-plane information of the structures, and the results are shown in Figure 4-8. The $\mathrm{RSM}$ results on the $\mathrm{STO}(013)$ plane shows that, for the temperature range considered, both the SRO layer and the LLTO matrix are epitaxially strained to the STO substrate as they present the same Qx values, while LMO, as shown by the RSM maps for the STO(113) plane, is relaxed, indicating that any change in unit-cell volume is caused by changes in composition or its structural interaction with the surrounding matrix.
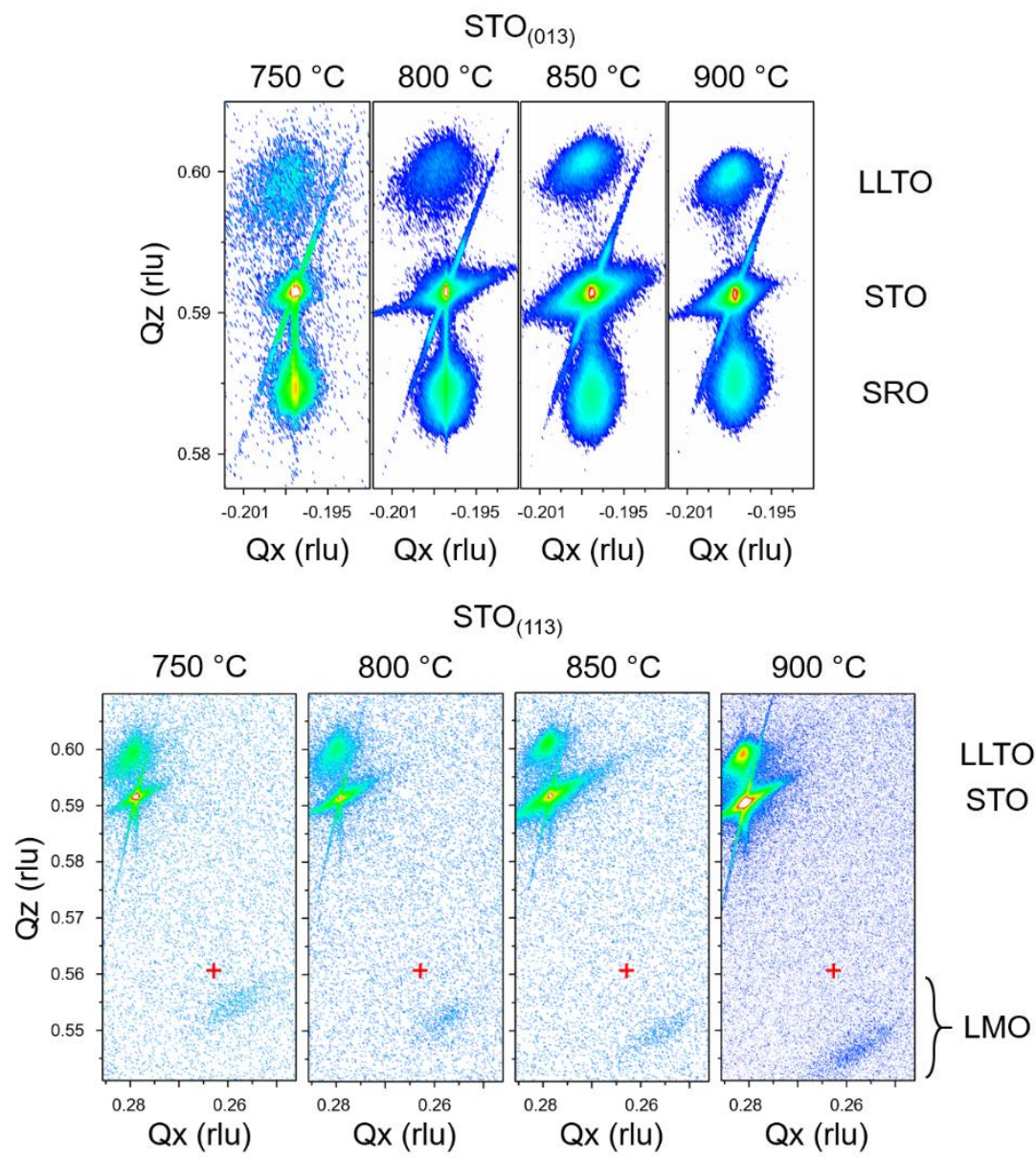

Figure 4-8. (top) RMS graphs obtained at the STO (013) plane and (bottom) RMS graphs obtained at the STO (113), the red cross indicates the position expected for the relaxed bulk LMO. 
The out-of-plane (c) and in-plane (a) lattice parameters were calculated considering a tetragonal unit cell from the RSM maps, and their values, including $c / a$ ratio and unit cell volume (V), are displayed in Figure 4-9. As observed by the out-of-plane analysis (Figure 4-7) the $c$ value of LMO increases with temperature, on the other hand, the $a$ value is reduced in an almost proportional way, with $c / a$ reaching unit for temperatures above 850 ${ }^{\circ} \mathrm{C}$. This compensation results in a nearly constant unit cell volume, of about $600 \AA^{3}, 7.1$ $\%$ larger than expected for bulk LMO, but that coincides for a $\mathrm{LiMn}_{2-\mathrm{y}} \mathrm{Ti}_{\mathrm{y}} \mathrm{O}_{4}$ phase with y $=1.5$.[45]
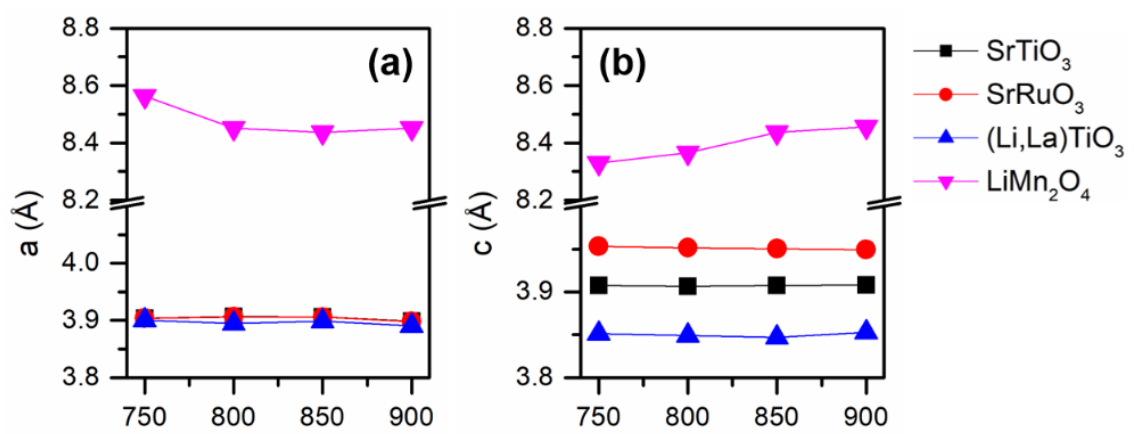

Temperature $\left({ }^{\circ} \mathrm{C}\right)$

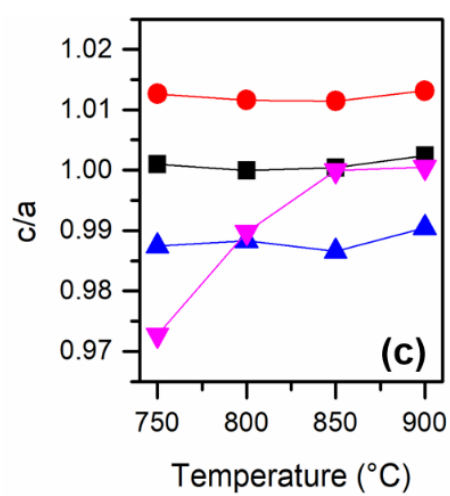

Temperature $\left({ }^{\circ} \mathrm{C}\right)$

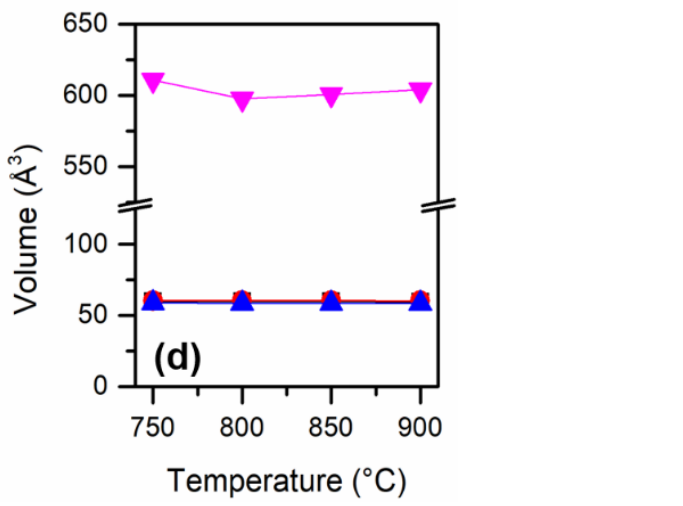

Figure 4-9. (a) In-plane and (b) out-of-plane lattice parameter for all components of the VAN films obtained from the RSM maps. (c) shows the c/a ratio and (d) the unit cell volume calculated considering a tetragonal unit cell and $\mathrm{a}=\mathrm{b}$ for all materials.

A more detailed structural and compositional analysis was performed through high resolution scanning transmission electron microscopy (HR-STEM) and electron energy loss spectroscopy (EELS), shown in Figure 4-10. Both perovskite LLTO and spinel LMO structures are visible in Figure 4-10a, on top of the perovskite SRO buffer layer. Initially considered to be exclusive to low frequencies, a 10 unit cell layer of LLTO between the LMO nanopillars and the SRO layer is also visible, which is not desired as an isolating 
layer can hinder the electronic transport between the cathode material and current collector. Figure 4-10b shows in more detail the LMO spinel structure of the pillars. With the annular bright-field (ABF) STEM, in which the contrast has a low scaling rate with the atomic number allowing simultaneous imaging of light and heavy elements, $\mathrm{Li}$ atoms within the structure are resolved. The EELS analysis, depicted in Figure 4-10d, shows the elemental composition along the line drawn in Figure 4-10c. The profile shows that, as discussed before, the pillars present a higher concentration of Ti of about 1.5 times higher than $\mathrm{Mn}$ and, in the region close to the LMTO/LLTO interface, an interphase with Ti/Mn $=2$ and some La intermixing into the pillars is observed. For the rest of the thesis, the LMTO phase would be referred as LMO for simplicity.

(a)

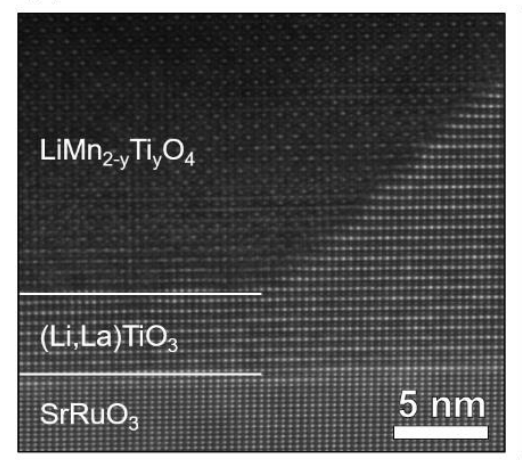

(b)

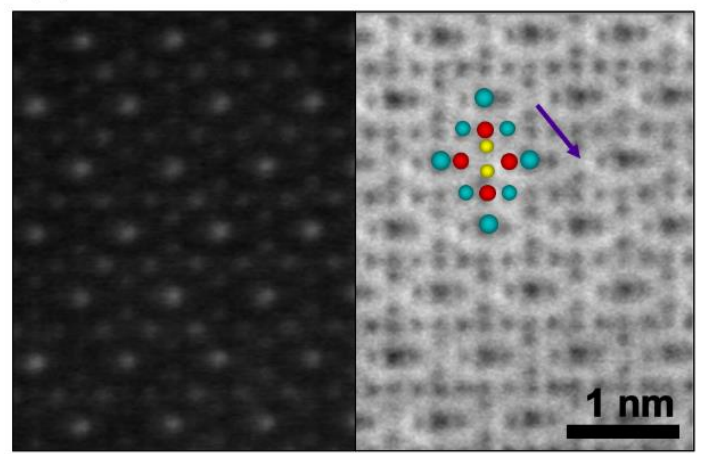

(d)

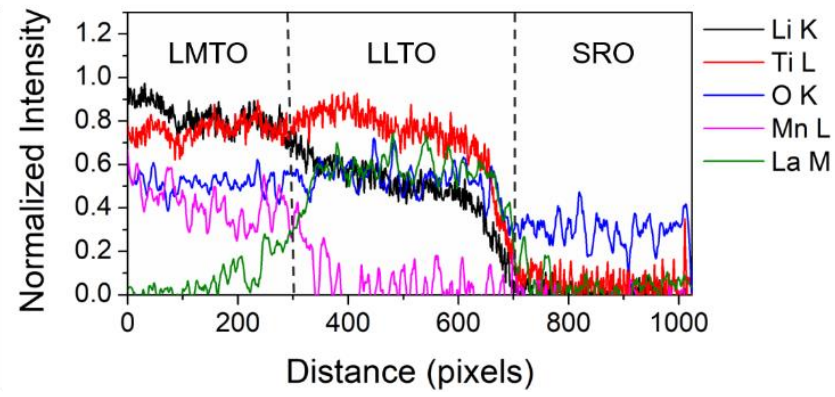

Figure 4-10. (a) HR-STEM cross section of the VAN thin film grown at $850{ }^{\circ} \mathrm{C}$, highlighting the 10 unit cells of LLTO between the LMO pillar and SRO layer. (b) Smaller range HR-TEM, with the respective annular bright-field image, highlighting the atomic structural arrangement of the $\mathrm{LiMn}_{2-\mathrm{y}} \mathrm{Ti}_{\mathrm{y}} \mathrm{O}_{4}$ pillars. The colorful spheres represent (light blue) $\mathrm{Mn} / \mathrm{Ti}$, (red) $\mathrm{O}$ and (yellow) $\mathrm{Li}$ atoms. Interstitial sites are showed by the purple arrow. Elemental analysis was performed with EELS on the profile highlighted in (c). (d) shows the normalized intensity for different elements along the line in (c). 


\subsubsection{Deposition Rate}

As described in chapter 3, kinetics play an important role on the size and distribution of the pillars in VANs,[36] although the deposition rate is not expected to influence the composition of the phases formed.[9] The PLD deposition of Li-containing materials, on the other hand, might be influenced by the deposition rate as higher pulse frequencies could limit the desorption of light elements from the substrate surface. For the analysis of the influence of deposition rate on the composition, structure and morphology of the desired VANs, a series of thin films were grown on $\mathrm{Nb}: \mathrm{STO}(100)$ substrates, at an oxygen pressure of 0.2 mbar, substrate temperature of $800{ }^{\circ} \mathrm{C}$, within the frequency range 8-50 Hz. A $50 \mathrm{~nm} \mathrm{SrRuO} 3$ (SRO) layer was deposited as an intermediate layer to enhance the electrical transport between the $\mathrm{LiMn}_{2} \mathrm{O}_{4}$ cathode pillars and the conducting $\mathrm{Nb}: \mathrm{STO}$ substrate.[34,35]
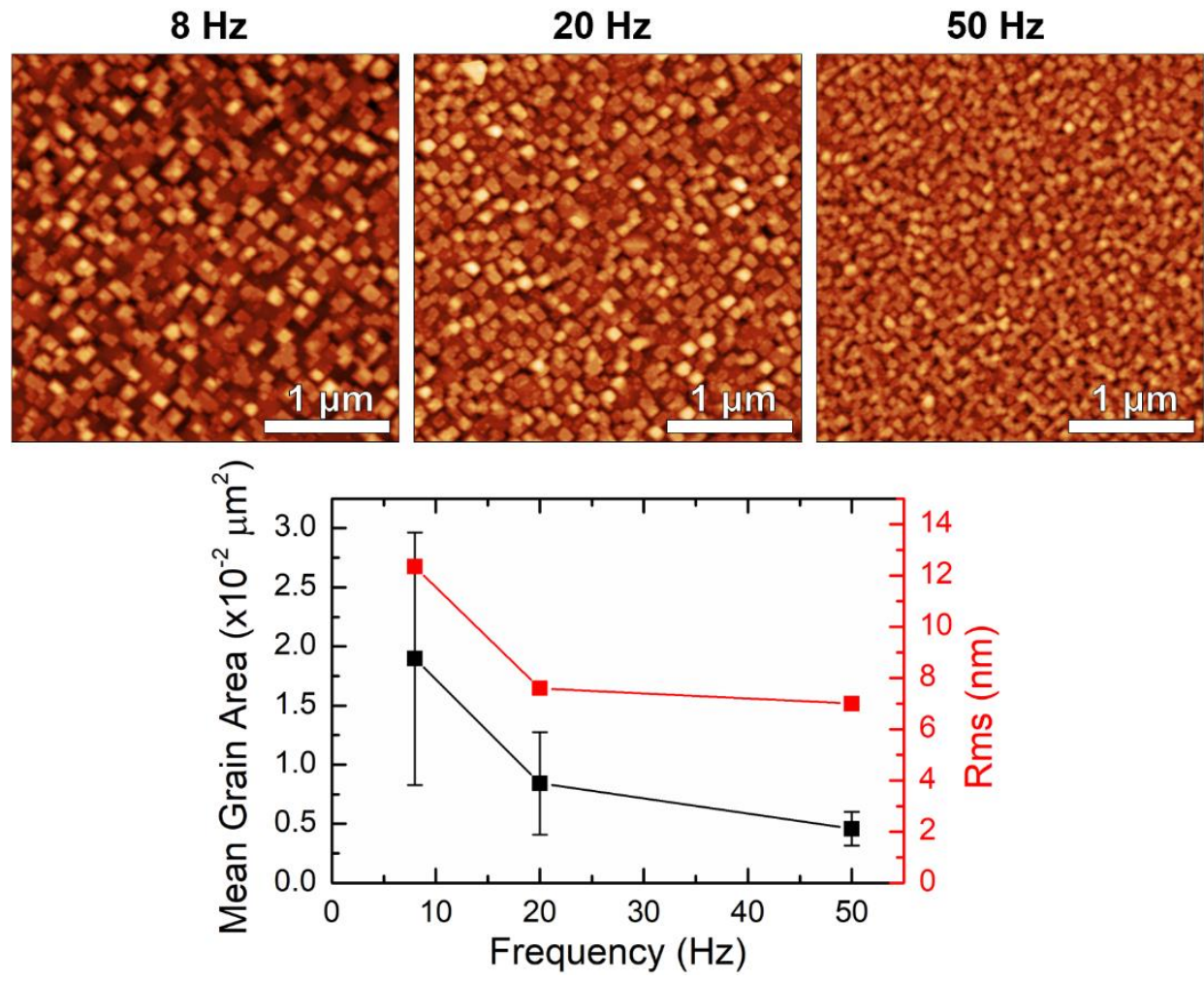

Figure 4-11. (top) AFM images for VAN grown at different deposition rates. (bottom) Mean grain area and Roughness RMS obtained from the AFM images.

The AFM images given by Figure 4-11 show the VAN morphology obtained for different deposition rates. Contrary to the temperature influence on the grains, higher 
frequencies limits the diffusion length by increasing the amount of collisions with the constant arrival of adatoms on the surface.[46,47] As presented by the mean grain area dependence on deposition rate, higher frequencies yields smaller, shorter, but more uniform pillars in accordance with the kinetic model presented in chapter 3 and other studies on the growth and formation of VANs.[9,36]
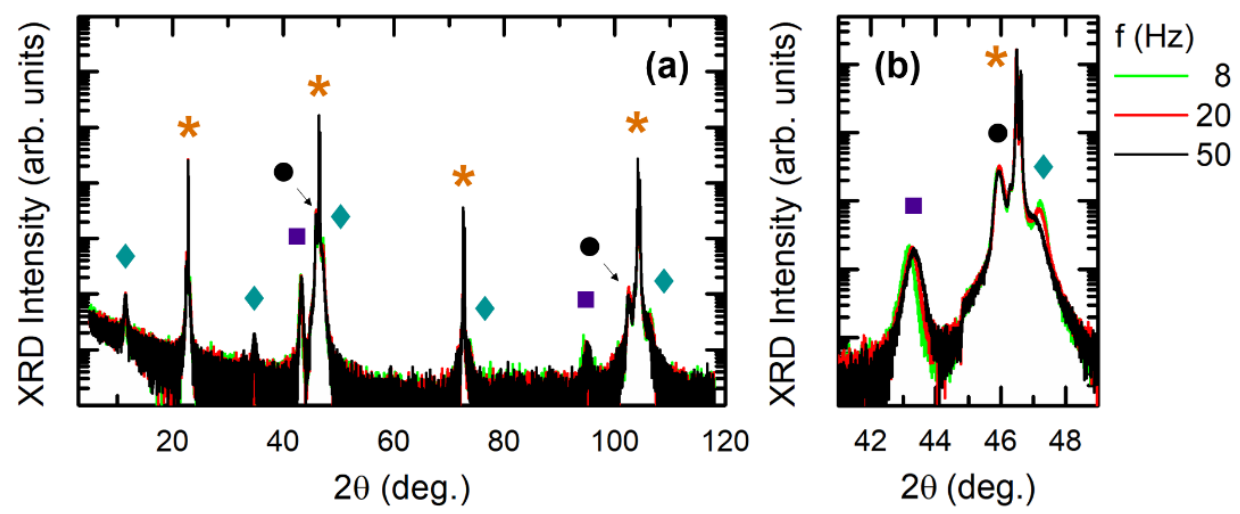

Figure 4-12. (a) Full XRD pattern of the VAN films grown at different frequencies, in which LLTO peaks are shown by $\bullet$, LMO by $\boldsymbol{\square}$, SRO by $\boldsymbol{\bullet}$, and the STO substrate peaks by $\star$. (b) XRD pattern highlighting the peaks between $40^{\circ}-50^{\circ}$.

Although it is known many materials require lower deposition rates to form the correct phase or smoother films, [47-49] for VAN formation it is expected that a change in frequency would have larger effect over the morphology, as discussed above, than on the composition of the phases formed.[9] On the other hand, as a light element, Li desorbs from the substrate surface on a higher rate as the other elements, [46] thus for higher frequencies would be expected to increase the amount of $\mathrm{Li}$ in the film, limited only by the Li contained in the target, while the Ti/Mn ratio should remain the same. Structural characterization of the VAN films grown at different deposition rate are shown in Figure 4-12. The LMO peak presents a shift from $8.38 \AA(8 \mathrm{~Hz})$ to $8.34 \AA(50 \mathrm{~Hz})$ on the frequency range analyzed, corresponding to a $1.4 \%$ reduction in the unit cell volume (considering a cubic unit cell). This change can be explained by variation in Li content in the cell, previously observed for LMTO cathode upon charge (up to $1.7 \%$ volume change), confirming this hypothesis.

\subsubsection{Substrate crystal orientation}

As aforementioned, VANs offer promising advantages as their functionalities can be tailored by the coupling at their vertical interfaces. However, the degree of coupling and hence the significance of such nanostructures is critically dependent on the nanostructure morphologies including domain patterns and shapes as well as structures and properties 
of the interfaces. The surface energy of spinels is very different from that of perovskites, as most perovskite phases are characterized by low-energy (100) surfaces, [50-52] while in spinels, the (111) planes exhibit the lowest surface energy.[34,53-56] The growth mode of the perovskite and spinel phases can, thus, be very different when grown on specific substrate surfaces. Therefore, different substrate orientations, are expected to modify the arrangement of pillars and matrix and, consequently, the electrochemical performance of the films.

To estimate the advantages of 3D electrode geometries over 2D planar films in terms of the contact area, a model was constructed. It is expected that by increasing the interfacial area while keeping the volume of the electrodes constant, an enhanced power density can be achieved. The model is based on values obtained by AFM images and the cross-sectional SEM data of all three orientations, presented in Figure 4-14, with support of models for VAN pillars found in literature.[9] The pillar-shape approximation models are displayed on Figure 4-13 top).

(100)

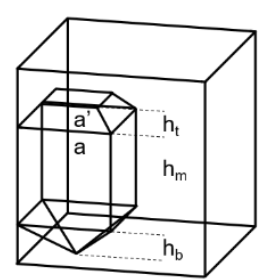

(110)
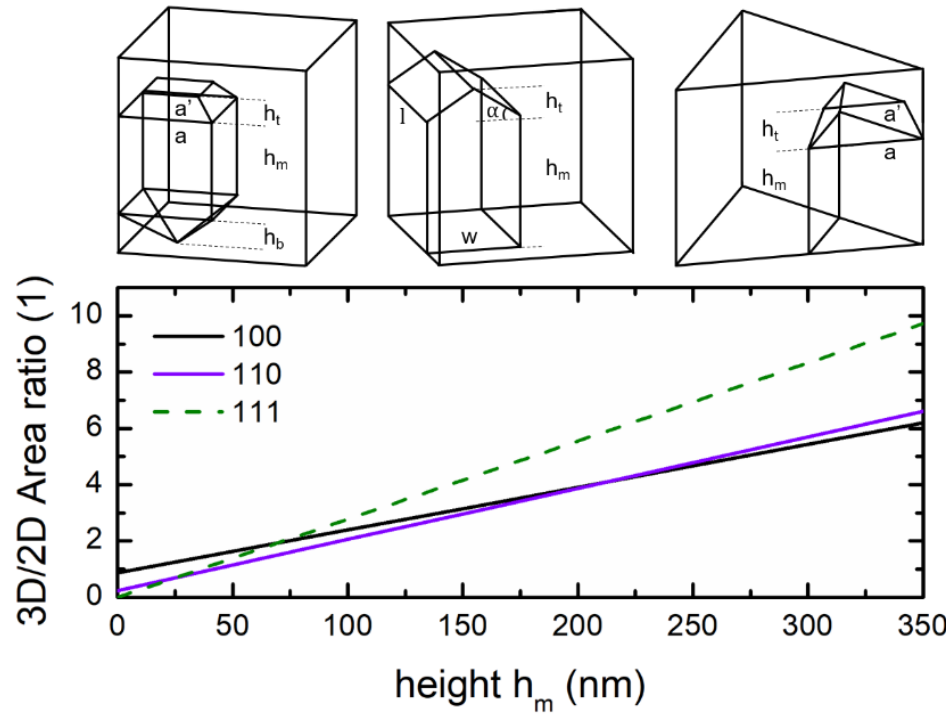

Figure 4-13. (top) Pillar model for different orientations and (b) 3D/2D area ratio dependence on the pillar's height $\left(\mathrm{h}_{\mathrm{m}}\right)$.

To determine the improvement in surface area, the $2 \mathrm{D}$ area was calculated considering the projection of the top contact of the pillars with the electrolyte, whereas the $3 \mathrm{D}$ area takes into account the height of the pillars $\left(\mathrm{h}_{\mathrm{m}}\right)$ and the shape of the base. For different film thickness, it is assumed that the bottom and the top section of the models in Figure 4-13(top) remain constant and that only $h_{m}$ changes. With the models considered, the following equations were used to estimate the total contact area for the nanopillars: 
(100)

$$
\begin{gathered}
A_{(100)}=4 \mathrm{a} \times h_{m}+2 a \times\left(h_{b}^{2}+\left(\frac{a}{2}\right)^{2}\right)^{1 / 2}+4 h_{t} \times \frac{a+a^{\prime}}{2}+a^{\prime 2} \\
A_{(110)}=2 \times h_{m} \times(w+l)+h_{t} \times w+2 \times \frac{h_{t}}{\cos \alpha} \times l \\
A_{(111)}=3 a \times h_{m}+h_{t} \times\left(3 \frac{a+a^{\prime}}{2}+0.5 a^{\prime}\right)
\end{gathered}
$$
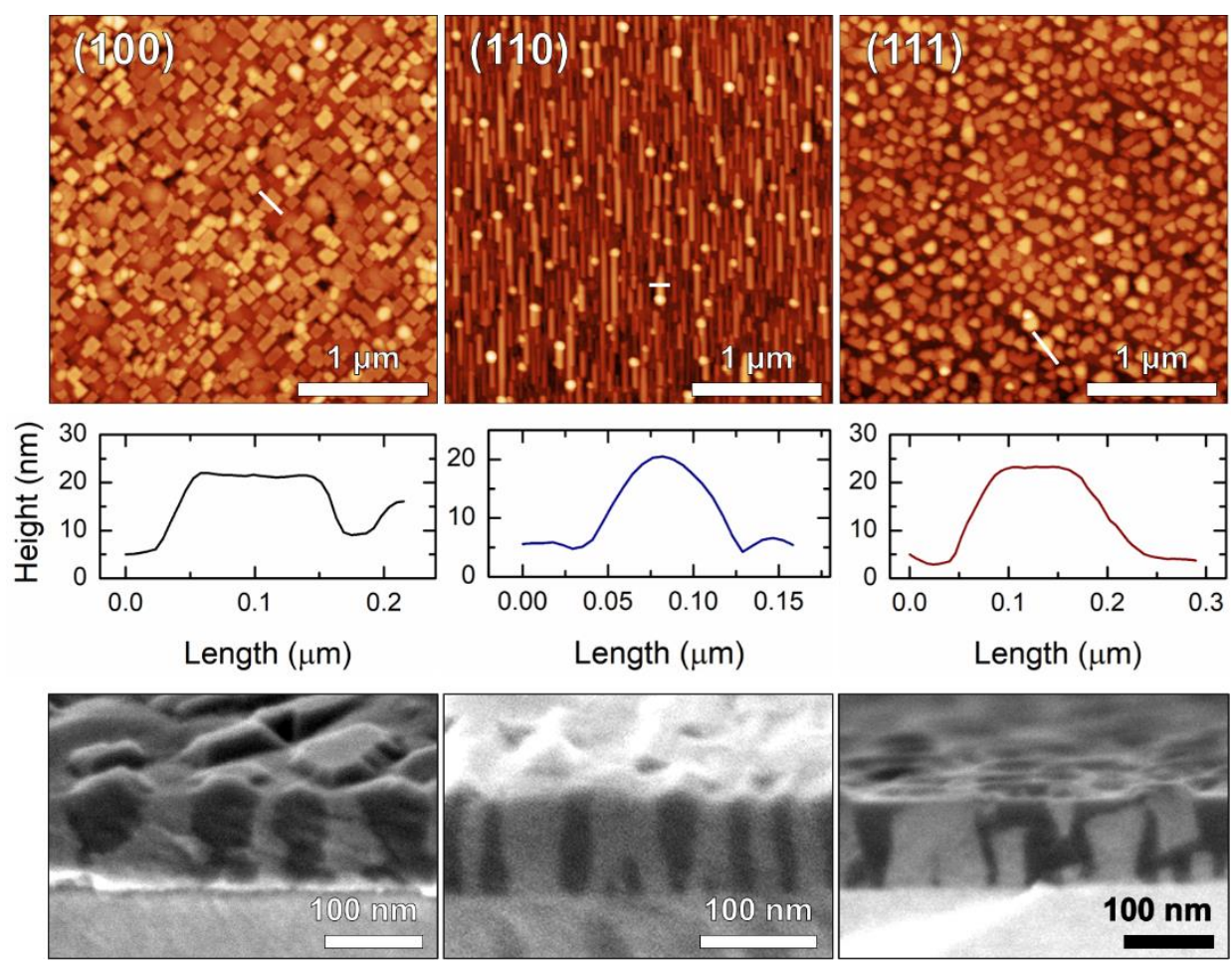

Figure 4-14. (top) AFM images of the VAN films grown under the same deposition conditions on $\mathrm{Nb}$ :STO substrates with different crystallographic orientations. (middle) Height profiles obtained from the AFM images on the regions highlighted in white. (bottom) Cross-sectional SEM showing the formation of pillars for different orientations.

All values, except for the pillar height $\left(\mathrm{h}_{\mathrm{m}}\right)$, in Equation 4.1, 4.2 and 4.3 were extracted from Figure 4-14. By altering $h_{m}$, the estimation of improvement in contact area for the 
VAN thin films depending on thickness is shown Figure 4-13(bottom). Surprisingly, the slope of the (111)-model is steeper than for the other two orientations, it is also possible to observe that the (110) model surpasses the (100) in surface area above $200 \mathrm{~nm}$ thickness due to its elongated profile, which makes it an interesting candidate for thicker battery electrode application.

To investigate the influence of crystal orientation on the structure and morphology of the desired VANs, a series of thin films were grown with a deposition rate of $8 \mathrm{~Hz}$, at an oxygen pressure of 0.2 mbar, substrate temperature of $800^{\circ} \mathrm{C}$, on $\mathrm{Nb}: \mathrm{STO}$ substrates with three different crystal orientations, namely (100), (110) and (111). The AFM images given by Figure 4-14 show the surface morphology of the VANs obtained for different substrate orientations. Although the different shapes obtained are clearly visible, specifically square-, rooftop- and triangular-like for the (100), (110), and (111) respectively, the AFM profiles show that the pillar structures do not vary significantly in height $(\sim 15 \mathrm{~nm})$ from the matrix baseline. This small variation keeps the roughness of the films nearly constant (RMS of $6.9 \mathrm{~nm}, 3.9 \mathrm{~nm}$ and $4.2 \mathrm{~nm}$ for the (100), (110) and (111), respectively) which is not observed for the individual materials grown in different substrate orientations, as described in chapter 2.[34,53]

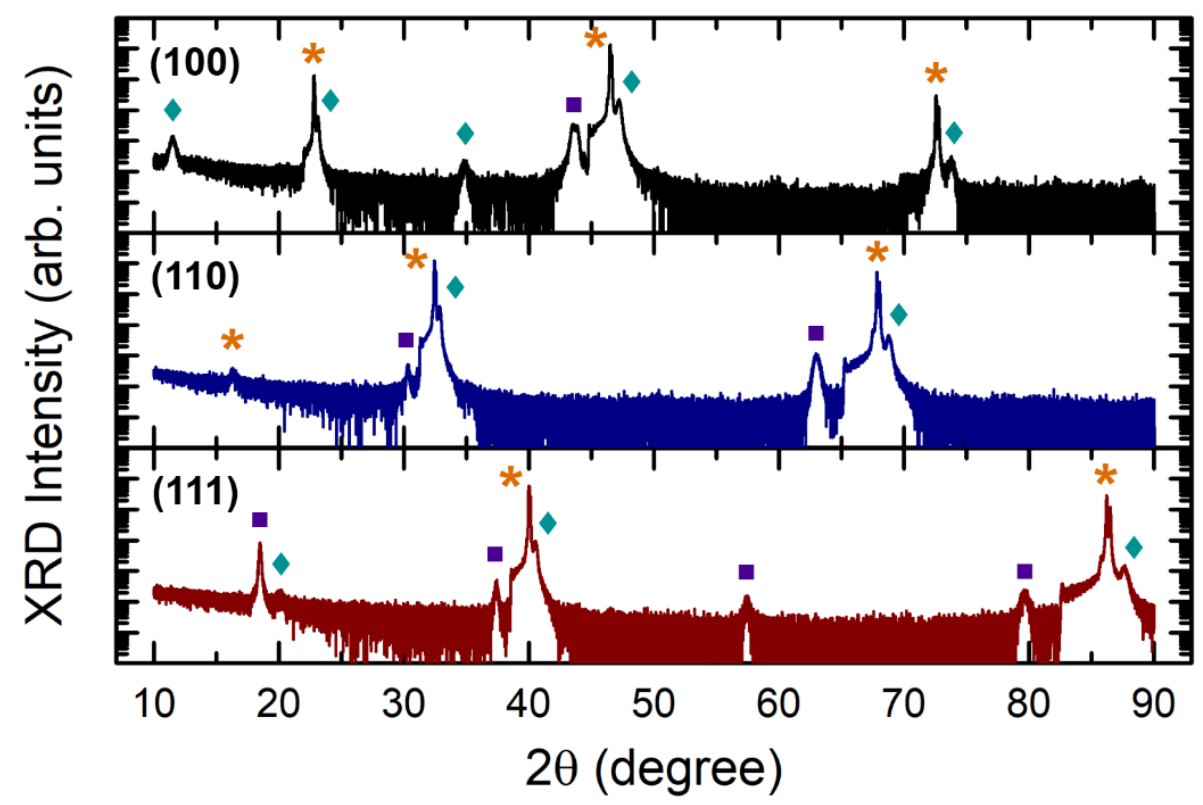

Figure 4-15. Full XRD pattern of the VAN films grown with the same conditions on STO substrates with different crystallographic orientations, in which LLTO peaks are shown by $\diamond$, LMO by $\boldsymbol{\square}$, and the STO substrate peaks by $\star$.

The cross-sectional SEM images show the formation of square and elongated pillars for the (100) and (110) crystallographic orientations, respectively, indicating that it is 
possible to tailor the pillar/matrix arrangements for Li containing materials as it has been reported for other systems. $[9,10,57]$ The triangular features showed by the AFM image for the VAN film grown on the (111) substrate orientation does not display uniform pillars underneath which could be a result of LLTO being grown with a tetragonal unit-cell, confirmed by the structural analysis in Figure 4-15. Other studies have shown a switching behavior in films grown in the (100) and the (111) orientation, where due the symmetry of the crystal structure, on different orientation the matrix become the pillar and vice versa. $[9,10,57]$ Such behavior is not observed here, which supports the proposition that a tetragonal unit-cell is present, which lacks the symmetry required for such switching.

Structural analysis of the VAN films grown on different substrate orientations is shown in Figure 4-15. The out-of-plane XRD patterns indicate that both the LMO and LLTO phases are stabilized within the nanocomposite thin film and aligned to the STO substrate orientation, without any impurity phase, suggesting that the PLD deposition process parameters (e.g. temperature, pressure, laser energy density, target composition) were optimized successfully to correct for any loss of volatile lithium during ablation, nucleation or growth. The LMO and LLTO peaks show the presence of highly crystalline oriented spinel and perovskite structures, in a good agreement with previous studies of individual LMO or LLTO thin films grown on STO(100) substrates.[34,58] The VAN structure seems to stabilize the LMO phase at high temperatures $\left(800{ }^{\circ} \mathrm{C}\right)$, normally not achievable in LMO thin films, [34] which could be a result of the epitaxial strain induced by the perovskite matrix, as observed for other systems.[59]

\section{Thickness dependence}

In most device technologies that rely on planar films grown on a single crystal substrate surface, films are strained to the substrate lattice up to a critical layer thickness, $t_{c}$, which is on the order of a few nanometers, depending on the amount of the misfit. Above $t_{c}$, misfit dislocations form to release the strain and above $50 \mathrm{~nm}$ significant strain relaxation occurs.[7,60] Self-assembled VAN structures show the advantage of not having an intrinsic thickness limitation for strain control, because the strain is controlled by the vertical interfaces and not the single interface with the substrate. For pillar diameters smaller or equal to $20 \mathrm{~nm}$, the substrate controls the strain only in the first $20 \mathrm{~nm}$ and after that, the vertical strain imposed at the vertical interfaces dominates the overall strain state of the film,[61] as there is insufficient pillar width to allow full relaxation by misfit dislocation formation.

In chapter 1, it was mentioned that while planar 2D solid-state thin-film batteries exhibit an undesirable energy $v s$. power balance, 3D electrodes would allow for a much better energy storage performance,[62-64] where electrodes could contain as much material as required for increased capacity without hindering the ionic diffusion between 
them. Another advantage mentioned is that the internal surface area between cathode, electrolyte and anode is enlarged, improving their current output. As shown in the previous section, different crystal orientations yield different VAN architectures, and the possibility of growing thicker films and the expected improvement in contact area over 2D planar films, is studied here. The films grown in this section follow the same recipe of those in the previous sections, but with four times the amount of pulses, yielding approximately $400 \mathrm{~nm}$ thick films.
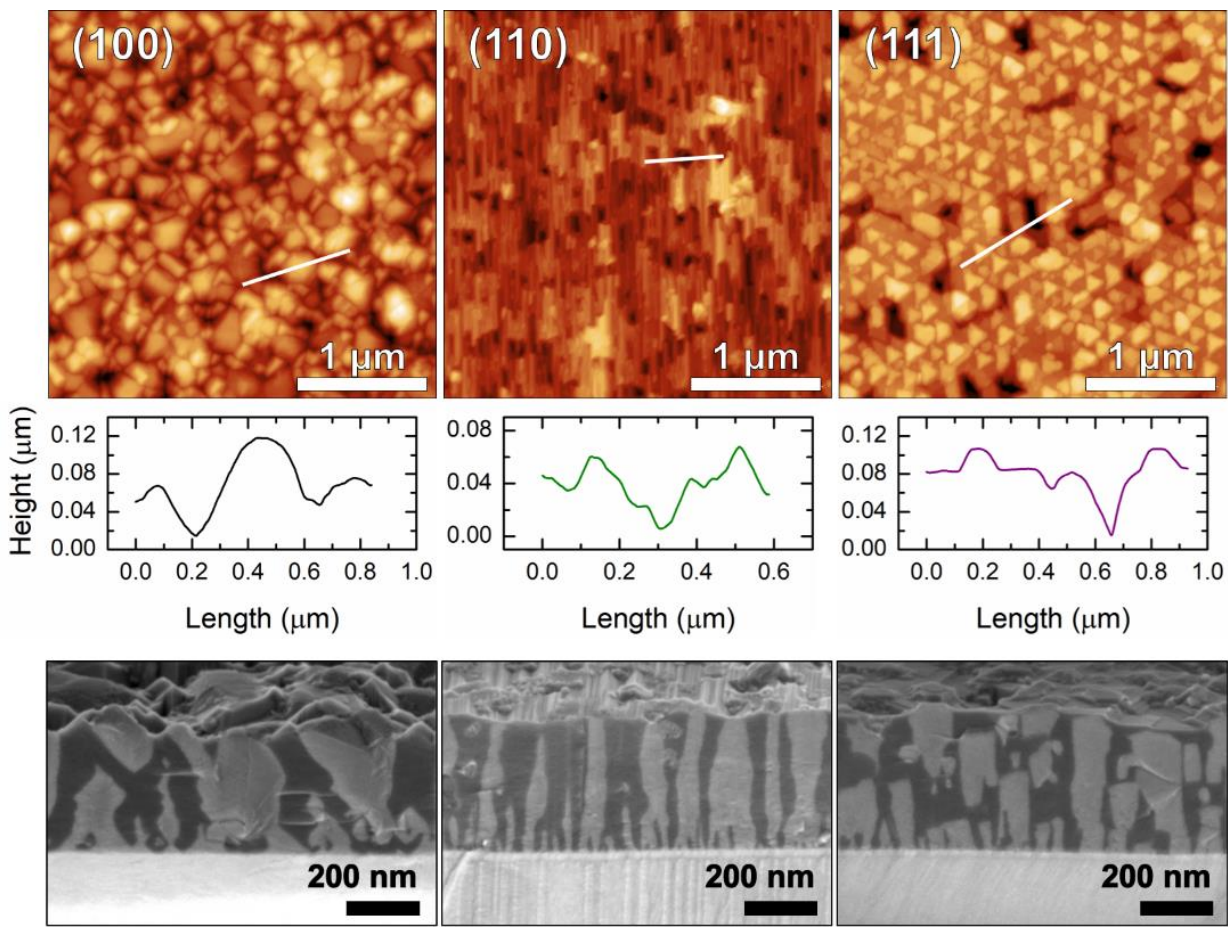

Figure 4-16. (top) AFM images of $400 \mathrm{~nm}$ VAN films grown with the same conditions on STO substrates with different crystallographic orientations. (middle) Height profile obtained from the AFM images on the regions highlighted with the respective colors. (bottom) Cross-sectional SEM showing the formation of coalesced pillars for different orientations.

The AFM images given by Figure 4-16 show the surface morphology of the thick VAN films obtained for different substrate orientations. The specific shapes observed previously in $100 \mathrm{~nm}$ thick VAN films are still visible for the thicker films, although for the (100) orientation the square-like features become less clear, while the triangular-like pillars for the (111) orientation become sharper. The height profile obtained from the AFM images show that the films become rougher (RMS of $22.5 \mathrm{~nm}, 11.07 \mathrm{~nm}$ and 13.6 $\mathrm{nm}$ for the (100), (110) and (111), respectively), which is expected for relaxed films. The 
relaxation is demonstrated by the cross-sectional SEM images at the bottom of Figure 4-16. As aforementioned, VANs improve the critical thickness $t_{c}$ of thin films for specific cases. For the VANs analyzed, the pillars maintain their shape for $\sim 110 \mathrm{~nm}$, above what is commonly obtained for thin films,[60] but as the structures relax with the increase in thickness, they tend to coalesce. The average pillar size of $\sim 80 \pm 20 \mathrm{~nm}$ for the (100) orientation, for example, could be sufficient to form misfit dislocations and allow the full relaxation of the films. Tuning the growth parameters to decrease the pillar size (e.g. higher frequencies, or manipulating the surface diffusivity) could improve the stability of pillar shape for thicker films of these nanocomposites.

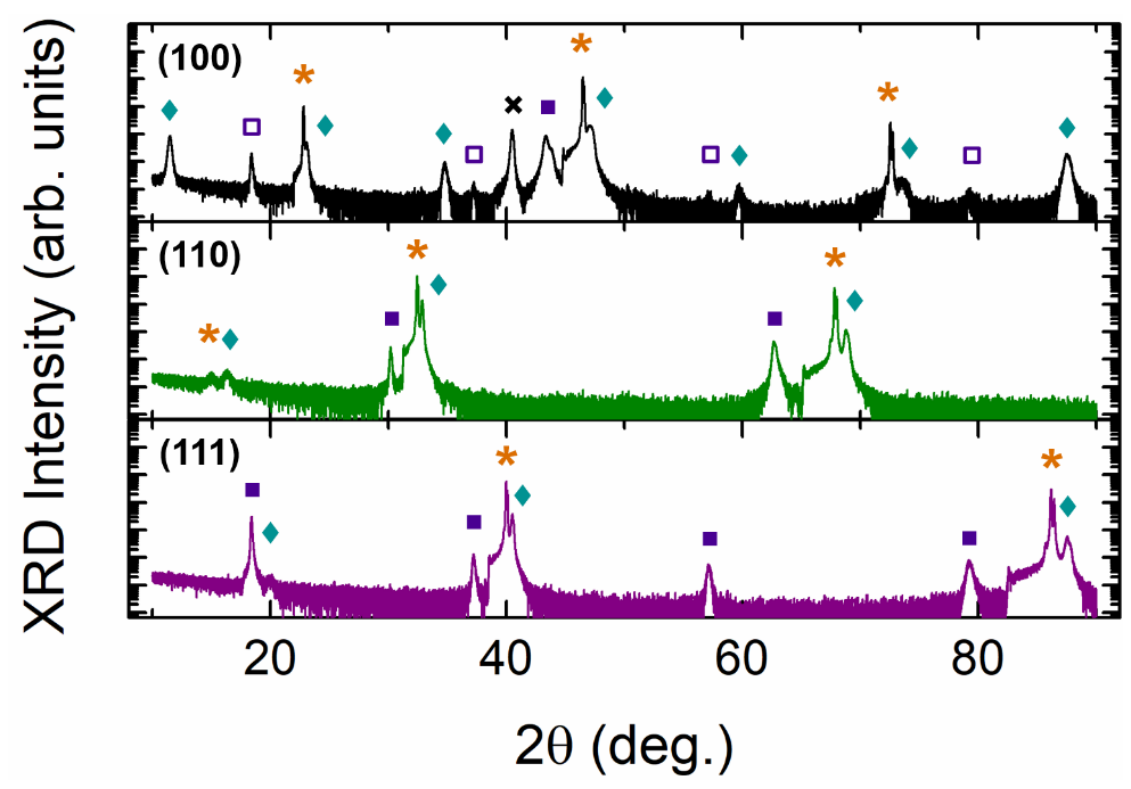

Figure 4-17. Full XRD pattern for $400 \mathrm{~nm}$ VAN films grown with the same conditions on STO substrates with different crystallographic orientations, in which LLTO peaks are shown by $\boldsymbol{\diamond}$, LMO by $\boldsymbol{\square}$, and the STO substrate peaks by $\star$. Impurities phases are indicated by $\square$, corresponding to $\mathrm{Mn}_{2} \mathrm{O}_{3}$ and $\boldsymbol{X}$, to rutile $\mathrm{TiO}_{2}$.

Structural analysis of the thicker films grown on different substrate orientations is shown in Figure 4-17. The out-of-plane XRD patterns indicate that both the LMO and LLTO phases are stabilized within the nanocomposite thin film and aligned to the $\mathrm{Nb}$ :STO substrate orientation. The LMO and LLTO peaks show the presence of highly crystalline oriented spinel and perovskite structures, in a good agreement with previous studies of individual LMO or LLTO thin films grown on STO(100) substrates.[34,58] It is also possible to observe additional peaks for the film grown on the (100) orientation which correspond to Li-deficient $\mathrm{Mn}_{2} \mathrm{O}_{3}$ and $\mathrm{TiO}_{2}$ phases. The coexistence of this lithium deficient phase could be due to the enhanced lithium volatility at the (100) surface of 
spinels, [65] as mentioned in chapter 2, that becomes more prominent on thicker films (and consequently longer deposition times). No impurity phases are present in these 400 nm thick (110)- and (111)- oriented VAN films.

\subsection{Investigating the influence of different conductive buffer layers}

In the previous sections, it has been mentioned that LMO requires a metallic layer as a buffer to the Nb:STO substrate to improve the electronic transfer at the interface.[34,35] The metallic layer reported in literature, and also used in most cases throughout this chapter is $\mathrm{SrRuO}_{3}$ (SRO). In order to investigate how different layers would play a role in the conductivity of the LMO pillars in the VAN proposed, in this section, different conductive buffer layers will be explored and their impact on the VAN growth will be investigated.

In chapter 3, the role of the interaction between different species with the substrate, in the form of activation energies for hopping, was investigated and their importance on their wetting behavior was unveiled, showing the important role of kinetics on the size and distribution of VAN structures.

The importance of different surface terminations on the growth of complex oxide materials was investigated the Solmaz et al.,[66] where it was observed that B-site terminated STO hinders the diffusion of BFO adatoms over the surface increasing the nucleation sites, while A-site terminated STO reduces the activation energy for hopping, yielding smoother films. The SRO layer used throughout this thesis results in a A-site terminated surface,[66] creating the need to investigate other terminations on the VAN growth.

To understand the influence of different substrate terminations on the growth of VANs, the KMCS model introduced in chapter 3 was used. All the Nb:STO substrates used throughout this thesis were treated to obtain the smooth $\mathrm{TiO}_{2}$ terraces on the surface.[67] Therefore the values obtained from the RHEED experiments are for a STO B-site terminated STO surface. On the study of self-organized SRO nanowires on ordered oxide surface terminations, [68] Kuiper et al. reported the activation energy for hopping on a Asite terminated surface is $\sim 67 \%$ lower than the activation energies for a B-site terminated substrate.[68] Thus, the activation energies for hopping for a A-site terminated surface for the materials used were calculated, based on the RHEED values obtained previously, and the new energies are presented in Table 4-1. 
Table 4-1. Activation energies obtained by RHEED for the B-site terminated films and the estimated activation energies for the A-site terminated surfaces. All the energies are provided in $\mathrm{eV}$.

\begin{tabular}{lccccc}
\hline Termination & $\mathbf{E}_{\text {LMO-STo }}$ & $\mathbf{E}_{\text {LMO-LMo }}$ & $\mathbf{E}_{\text {LLto-LMo }}$ & $\mathbf{E}_{\text {LLto-Sto }}$ & $\mathbf{E}_{\text {LLto-LLto }}$ \\
\hline A-site & $0.17 \pm 0.01$ & $0.34 \pm 0.01$ & $0.17 \pm 0.01$ & $0.32 \pm 0.01$ & $0.34 \pm 0.01$ \\
\hline B-site & $0.25 \pm 0.01$ & $0.51 \pm 0.01$ & $0.25 \pm 0.01$ & $0.49 \pm 0.01$ & $0.51 \pm 0.01$ \\
\hline
\end{tabular}

With the new values as an input for the KMCS model, the influence of different terminations on the VAN formation can be observed in Figure 4-18. For the A-site terminated substrate bigger grains are visible, whereas the B-site terminated substrate, smaller grains are presented, confirming the hypothesis. The model suggests that different activation energies would influence the contact point between the LMO pillars and the layer underneath. Comparing the pillars present on the edge of both images in Figure 4-18 it is possible to verify that on the A-site terminated surface, from all the pillars present, not all have a good contact with the bottom layer, while in the B-site terminated surface they are more noticeable.

(a)

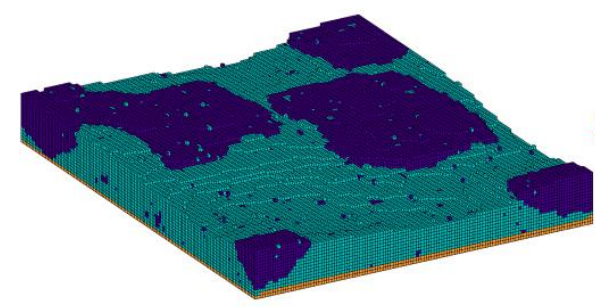

A-site terminated (b)

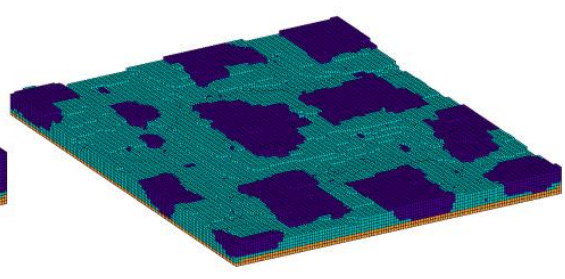

B-site terminated

Figure 4-18. 3D view of KMCS results for the LMO-LLTO nanocomposite growth for different (a) A-site and (b) B-site terminated substrates.

\subsubsection{Buffer layer candidates}

Promising candidates for replacing A-site terminated SRO as a bottom electrode are the B-site terminated $\mathrm{LaAlO}_{3}(\mathrm{LAO})$ and $\mathrm{LaNiO}_{3}$ (LNO). Both materials present the perovskite structure with LNO displaying a pseudocubic unit cell lattice parameter of 3.84 $\AA$ and resistivity of $90 \mu \Omega \mathrm{cm}$,[69] while LAO, has a pseudocubic lattice parameter of $3.79 \AA$ and band-gap of 5.5-6.5 eV [70] (insulator) but when grown on the STO, the interface can become conductive for very thin layers. The study of the buffer layers was 
carried out by PLD on $\mathrm{TiO}_{2}$-terminated $\mathrm{Nb}$ :STO substrates with the (100) orientation, using the parameters summarized in Table 4-2.

Table 4-2. Parameters for deposition of the different buffer layer materials studied in this chapter.

\begin{tabular}{llll}
\hline & \multicolumn{1}{r}{ SRO } & LAO & \multicolumn{1}{c}{ LNO } \\
\hline Temperature $\left({ }^{\circ} \mathrm{C}\right)$ & 600 & 800 & 700 \\
Laser frequency $(\mathrm{Hz})$ & 2 & 1 & 2 \\
$\mathrm{O}_{2}$ Pressure $(\mathrm{mbar})$ & 0.133 & 0.002 & 0.040 \\
Thickness $(\mathrm{nm})$ & 20 & 1.6 & 20 \\
Fluency $\left(\mathrm{J} \cdot \mathrm{cm}^{-2}\right)$ & 2.33 & 1.33 & 1.90 \\
Spot size $\left(\mathrm{mm}^{2}\right)$ & 1.92 & 1.92 & 1.92 \\
\hline
\end{tabular}
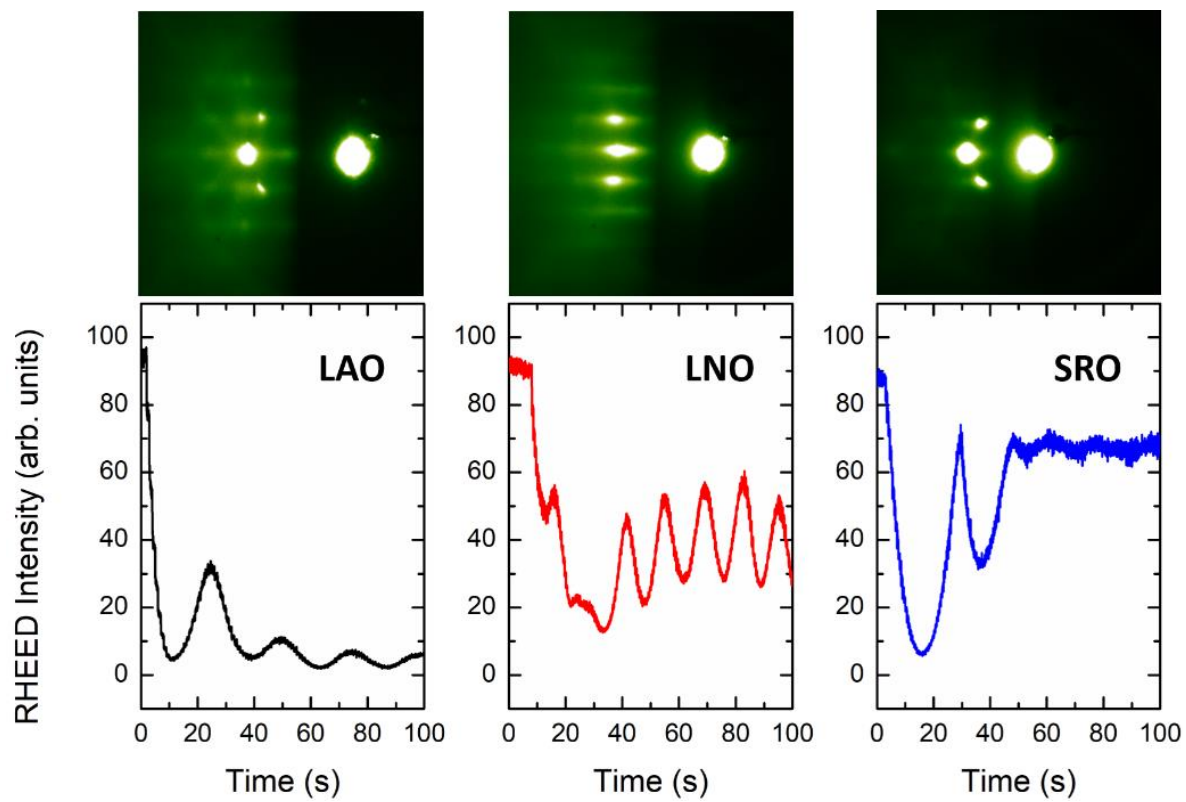

Figure 4-19. (top) RHEED pattern obtained at the end of the deposition and (bottom) RHEED intensity oscillations as a function of time for the different buffer layer materials.

To guarantee the growth quality the deposition process was monitored by RHEED, and the intensity oscillations and patterns are displayed in Figure 4-19. The RHEED images obtained at the end of the buffer layer deposition, displayed on Figure 4-19(top) show the characteristic diffraction patterns expected for the materials deposited, and ensures the smoothness of the layers, which is required for the subsequent deposition. The RHEED intensity oscillations in Figure 4-19(bottom) show the control of the layer thickness by tracking the layer-by-layer growth, as 4 monolayers are required for LAO, and for LNO and SRO the oscillations provide the growth rate used to calculate the layers thicknesses. 
The oscillations for the LNO layer show an erratic behavior for the first 50 pulses that could due to the specific alignment of the RHEED.

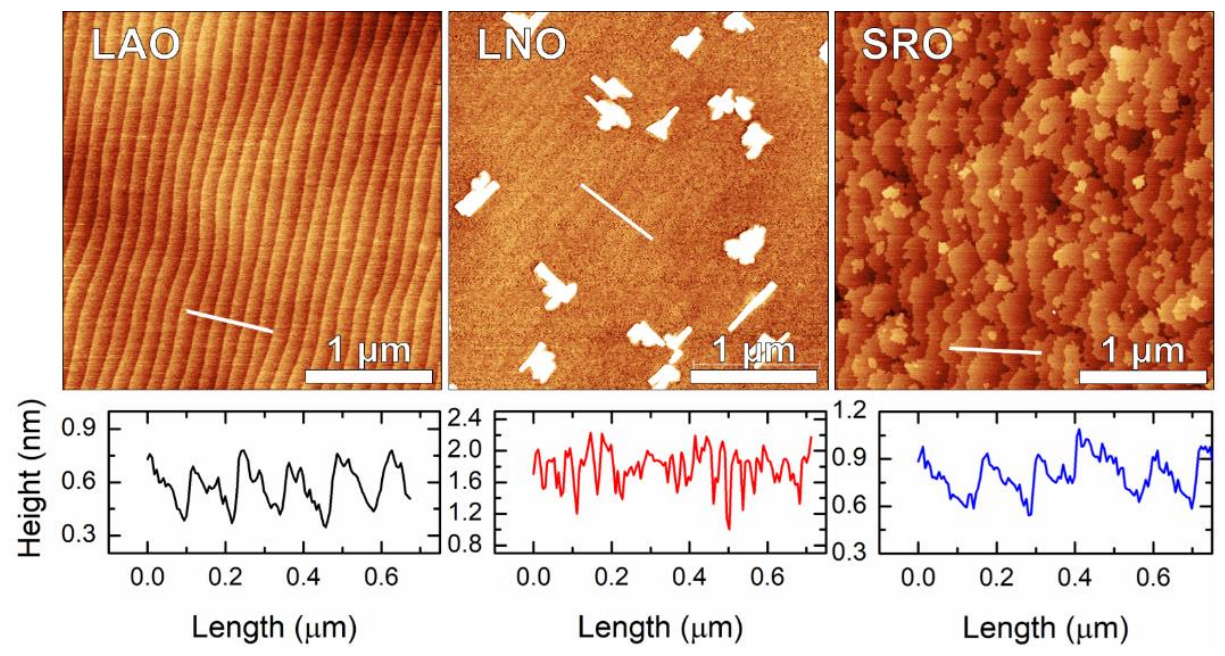

Figure 4-20. (top) AFM images of the different buffer layers grown on STO(100) substrates. (bottom) Height profile obtained from the AFM images on the regions highlighted in white.
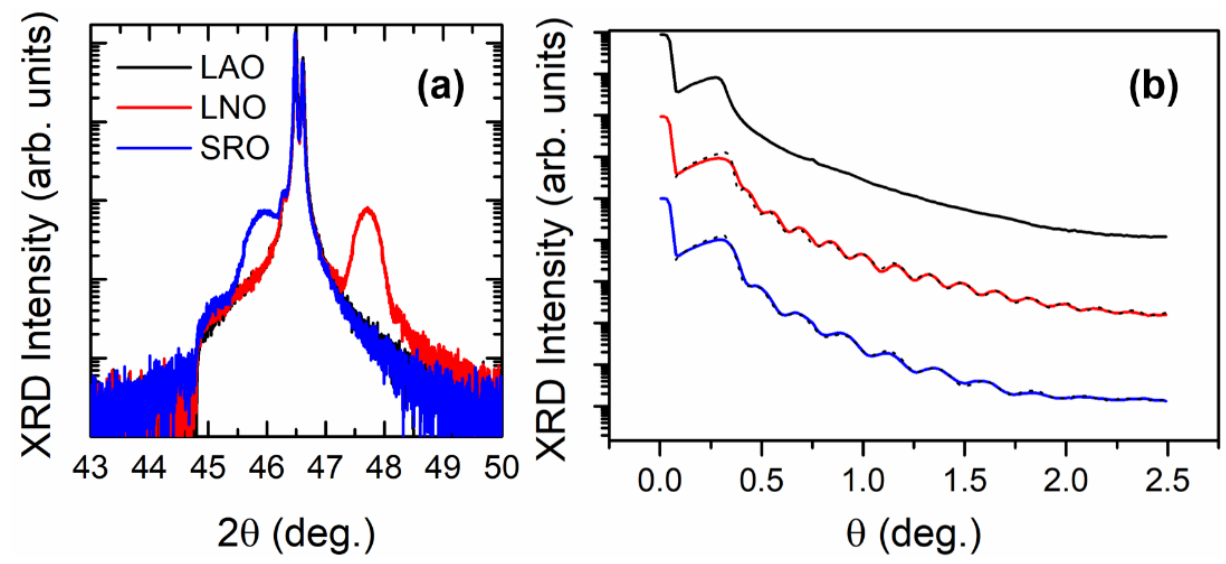

Figure 4-21. (a) XRD pattern of the different buffer layers thin films grown on STO(100) highlighting the peaks between $43^{\circ}-50^{\circ}$. (b) XRR measurements of the different buffer layers thin films. The dotted lines show the accuracy of the fit.

The AFM images given by Figure 4-20 show the surface morphology of the buffer layers grown. The layers obtained for all three materials display visible terraces and smooth surfaces with roughness RMS of $0.13,0.28$ and $0.20 \mathrm{~nm}$ for LAO, LNO and SRO, respectively, confirmed by the height profiles. The LNO layer morphology exhibits, 
however, features that seem to be aligned either perpendicular or parallel to the terraces, suggesting they might be crystalline and byproducts of an non-stochiometric growth.[71]

Out-of-plane structural analysis of the buffer layers is shown in Figure 4-21a. The XRD patterns indicate that the thin films are aligned to the STO substrate orientation, and the peaks show the presence of highly crystalline oriented perovskite structures, with lattice parameters of $\sim 3.95 \AA$ and $\sim 3.81 \AA$ for the SRO and LNO layers, respectively. The smaller out-of-plane lattice parameter observed for LNO, against the expected 3.86 $\AA$,[69] suggests that the layer is still strained to the substrate.

Reflectivity measurements, depicted in Figure 4-21b confirms the smoothness of the interfaces, and the fitting yields thicknesses of 18.3 and $25.8 \mathrm{~nm}$ for SRO and LNO layers, respectively, in agreement with the RHEED oscillations, and an average density of 5.45 g. $\mathrm{cm}^{-3}$. The volume of the LAO layer is insufficient to show any diffraction peaks. However, previous studies of LAO grown on STO have demonstrated full in-plane epitaxial ordering.[72]

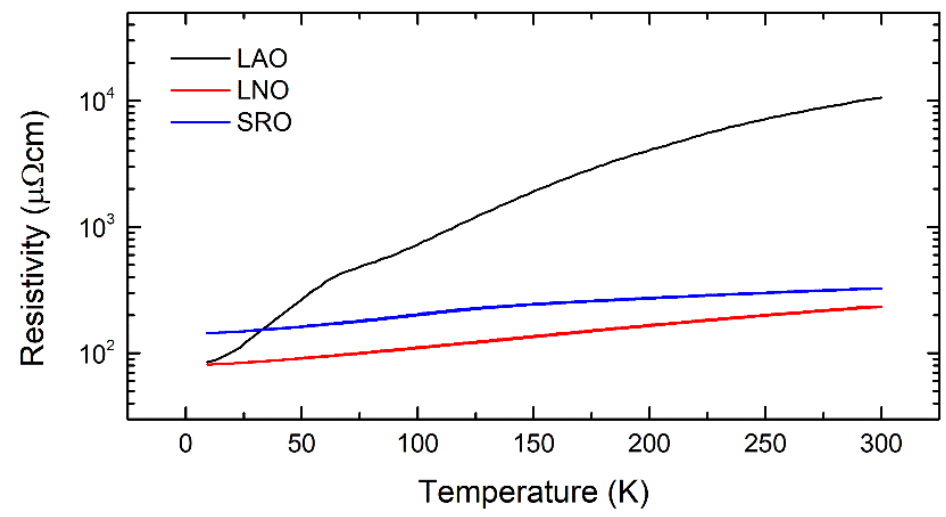

Figure 4-22. In-plane resistivity values measured for the different buffer layers thin films at low temperatures.

The different buffer layers are used to modify the diffusivity of adatoms over the surface and, although the structure and morphology are important properties, they are also meant to improve the electrical contact between the cathode nanopillars and the current collector. Thus, the resistivity of the layers were measured in-plane for different temperatures and the results are displayed in Figure 4-22. Resistivity values were calculated by multiplying the sheet resistances with the layer thicknesses, where $20 \mathrm{~nm}$ was used for the SRO and LNO layers, while $1.6 \mathrm{~nm}$ was used for the LAO layer. The resistivity values obtained for the samples analyzed agree with those observed in literature for thin films grown on the same conditions.[73-75] The characteristic transition temperature $\left(\mathrm{T}_{\mathrm{C}}\right)$ for $\mathrm{SRO}$, at $\sim 150 \mathrm{~K},[74]$ is noticeable. The conductivity of the LAO layer is taken as an estimate for comparison, as the $2 \mathrm{D}$ electron gas located at the interface 
for the LAO-STO system also expands over some thickness, making the LAO layer likely to show inhomogeneous conductivity.

\subsubsection{VAN formation on different buffer layers}

To evaluate the influence of different surface termination on the composition, structure and morphology of the desired VANs $\left(\mathrm{LiMn}_{2} \mathrm{O}_{4}+(\mathrm{Li}, \mathrm{La}) \mathrm{TiO}_{3}\right)$, a series of thin films were grown on $\mathrm{Nb}: \mathrm{STO}(100)$ buffered with the layers discussed above, at an oxygen pressure of 0.2 mbar, substrate temperature of $850{ }^{\circ} \mathrm{C}$, and deposition rate of $20 \mathrm{~Hz}$. The AFM images given by Figure 4-23 show the top morphology of the VAN structures grown on different buffer layers. It can be observed that different surface terminations do not impede the phase separation for the formation of VANs, as the pillar structures are visible for all three films. The strained LAO layer [72] exhibit a smoother surface than its counterparts, resulting in a smoother VAN film on the LAO layer, with roughness RMS of $5.1 \mathrm{~nm}$. The VANs grown on SRO and LNO layers show roughness values of 12.7 and $13.6 \mathrm{~nm}$, which are in the same range as the films grown previously with similar deposition parameters (Figure 4-11).
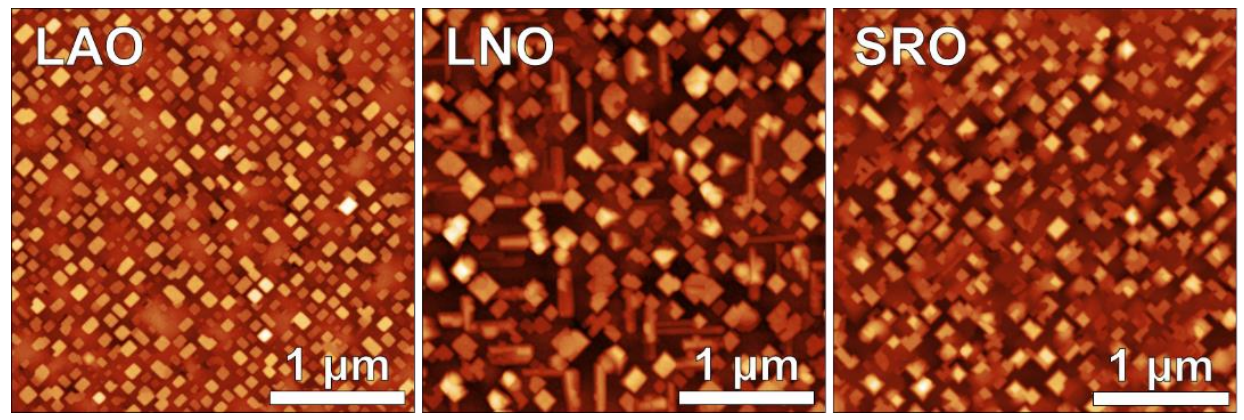

Figure 4-23. AFM images of the VAN thin films grown on different buffer layers.

The simulations presented by Figure 4-18 suggested that B-site terminated surfaces would yield VANs with smaller pillar sizes which is correct for the LAO layer, displaying average pillar size of $0.012 \pm 0.003 \mu \mathrm{m}^{2}$ compared to $0.018 \pm 0.005 \mu \mathrm{m}^{2}$ showed by the VANs on SRO buffer layer. For the VANs on LNO, however, the average pillar size is increased to $0.021 \pm 0.011 \mu \mathrm{m}^{2}$ with a high distribution of pillar sizes. This could be an effect of the presence of different crystallographic orientations or a more complex interaction between the VAN materials and the LNO layer.

Out-of-plane structural analysis of the VANs on different buffer layers is shown in Figure 4-24. The XRD patterns show all the peaks expected for the LMO phase and tetragonal LLTO, indicating that different surface terminations do not have influence on 
the crystal structure of the VAN materials. The VAN film grown on LAO shows the highest XRD intensity LMTO and LLTO peaks, which could be result of the strained starting surface provided by the LAO layer underneath improving the crystallinity of the LLTO layer. The peaks positions change depending on the underlying layer: $47.08^{\circ}$, $47.17^{\circ}$ and $47.19^{\circ}$ for the LLTO peaks and $43.12^{\circ}, 43.06^{\circ}$ and $43^{\circ}$ for the LMTO peaks for the LAO, LNO and SRO layers, respectively. The shift to higher angles for the LLTO peaks could be attributed to the interaction of the films and the buffer layers, which display increasing lattice parameter of $3.79 \AA$, $3.86 \AA$ and $3.91 \AA$, that better accommodate the matrix. The pillars follow the inverse trend. Small peaks are visible at $6.83^{\circ}$ and $42^{\circ}$ which corresponds to $\mathrm{La}_{2} \mathrm{Ti}_{2} \mathrm{O}_{7}$. The $\mathrm{LNO}$ buffer layer displays small peaks related to $\mathrm{LMTO}(110)$ at $30^{\circ}$ and $62^{\circ}$, which could be the influence of the irregular starting LNO layer (Figure 4-20).
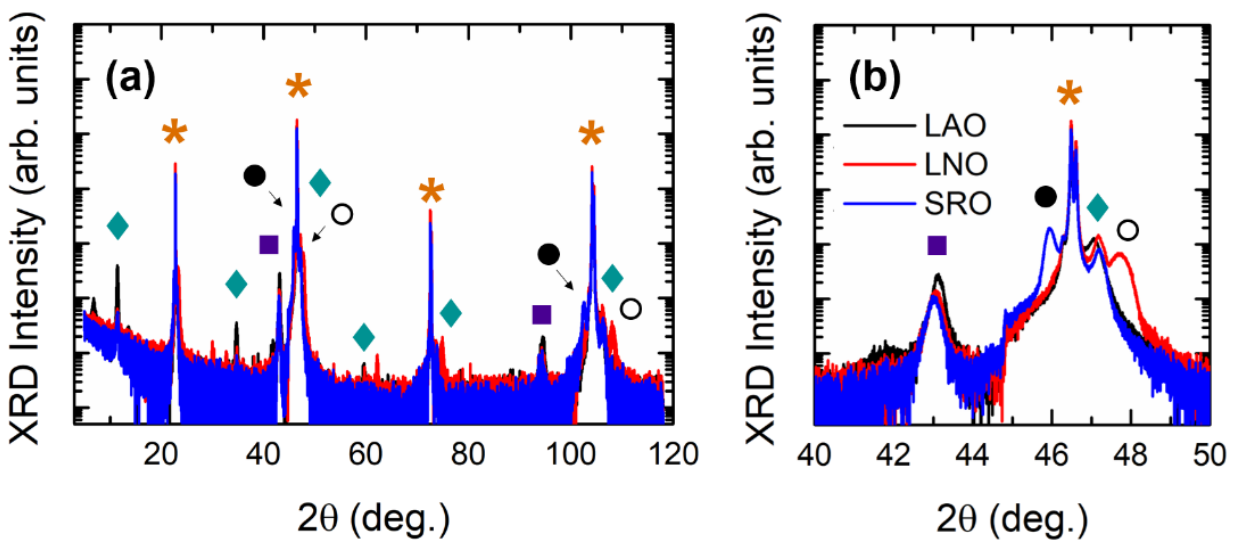

Figure 4-24. (a) Full XRD pattern of the VAN films grown on buffer layers with different terminations, in which LLTO peaks are shown by $\bullet$, LMO by $\boldsymbol{\square}$, SRO by $\boldsymbol{\bullet}$, LNO by $\mathrm{O}$ and the STO substrate peaks by $\star$. (b) XRD pattern highlighting the peaks between $40^{\circ}-50^{\circ}$.

The VAN grown on LNO was analyzed in more detail by HR-STEM (Figure 4-25b) to verify if what was observed from the simulations that the diffusion of adatoms on B-site terminated surfaces would improve the contact between the pillars and the current collector. The HR-STEM image in Figure 4-25a is the same showed previously in Figure 4-10a, as a comparison. It is possible to observe that, although both the LMTO and LLTO maintain their spinel and perovskite structure, respectively, the LMTO pillars incorporates into the LNO layer, indicated by the white arrow in Figure 4-25b. 

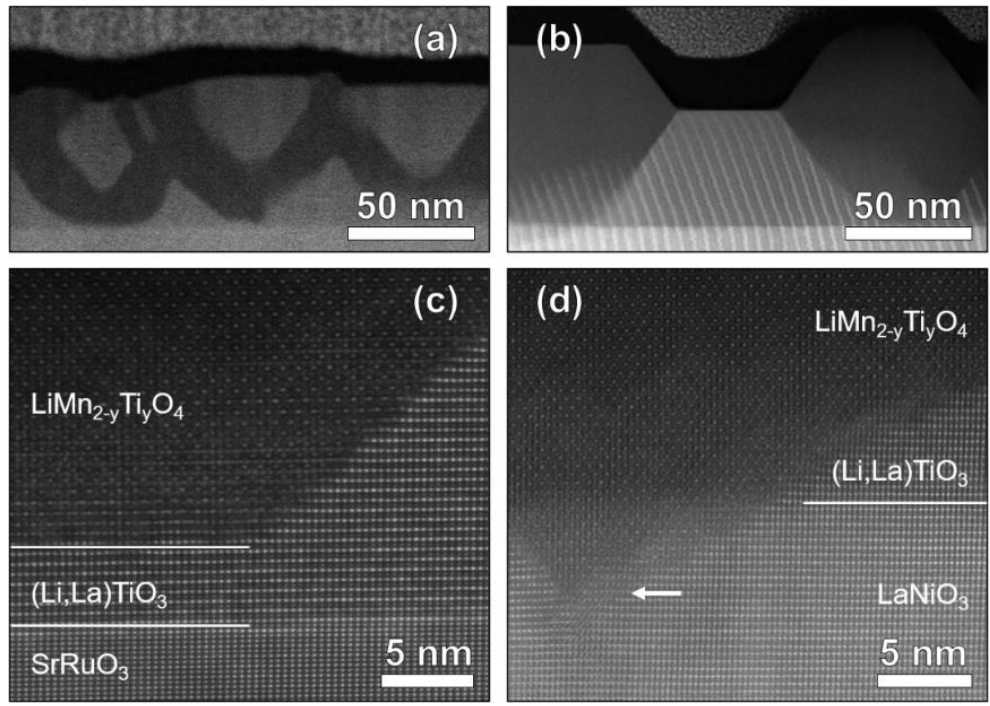

Figure 4-25. HR-STEM cross section of the VAN thin film (a) on SRO layer and (b) on LNO layer. Higher magnification of HR-STEM cross section of the VAN thin film (c) on SRO layer, highlighting the 10 u.c. of LLTO between the LMO pillar and SRO layer, and (d) on LNO layer, where the white arrow indicates the point where the spinel incorporates into the LNO buffer layer.
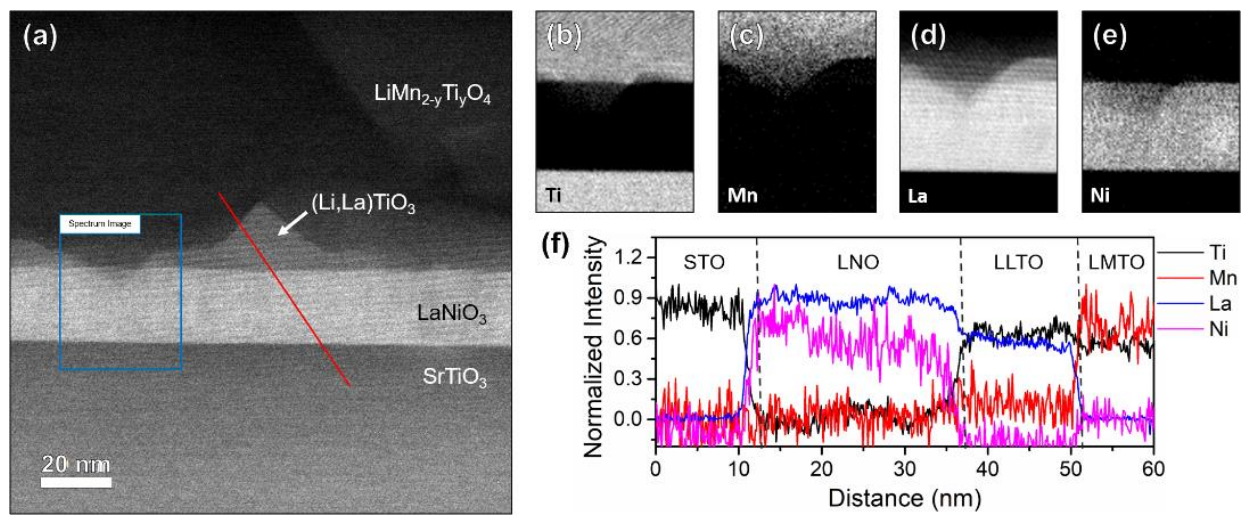

Figure 4-26. (a) HR-STEM cross section of the VAN thin film on the LNO layer. Elemental analysis was performed with EELS on the profiles highlighted by the blue box and red line in (a). (b-e) show the distribution of different elements on the region in the blue box in (a). (f) shows the normalized intensity for different elements along the red line in (a). 
The incorporation of the LMTO pillars into the LNO layer could indicate that no LLTO is formed in between the pillars and the buffer, improving its conductivity. To better understand the interface between the layers of the VAN grown on LNO, elemental analysis was performed with EELS, shown in Figure 4-26. The cross-section in Figure 4-26a show the LNO and LLTO/LMO layers grown on Nb:STO. The region highlighted by the blue box was analyzed for different elements and the results are displayed in Figure 4-26b-e. As expected, Ti is present on the STO substrate and on the LLTO and LMTO portions of the film, as also shown in the profiles in Figure 4-26f. Figure 4-26d shows La present on the LNO film and the LLTO portion of the nanocomposite, Figure 4-30e shows $\mathrm{Ni}$ is present only on the LNO layer. Analyzing closely the incorporated pillar portion of the image, it is noted that the Ti in Figure 4-26b goes deeper into the LNO layer than the Mn in Figure 4-26c. This asymmetry suggests that a LLTO layer is still being formed in between the LMTO pillar and the buffer layer. The line profile in Figure 4-26e shows that the $\mathrm{LiMn}_{2-\mathrm{y}} \mathrm{Ti}_{\mathrm{y}} \mathrm{O}_{4}$ phase is being formed with $\mathrm{y}=1$.

Lastly, the formation enthalpy of spinel decreases drastically $\left(\Delta \mathrm{H}=-121.86 \mathrm{~kJ} \mathrm{~mol}^{-1}\right)$ with Ni doping, indicating that LMNO has a higher structural stability than that of the pristine and, therefore, is thermodynamically more favorable to form.[76] The absence of $\mathrm{Ni}$ in the pillars, in Figure 4-26, shows the stability of the LNO layer with the LMO/LLTO nanocomposite combination.

\subsection{Silicon}

Since the microelectronic industry is based on the fabrication of devices on silicon wafers, integration of VANs on silicon is required for the integration of these structures as microbatteries.[77] Growth of epitaxial oxides on silicon is not straightforward as the unavoidable presence of an amorphous silicon native oxide prevents a lattice match between the silicon lattice and the orientation of the growing film. To verify if the VAN thin films for battery application could be grown on Si substrates, and how that would influence the composition, structure and morphology of the desired VANs, a thin film was grown on $\mathrm{SrTiO}_{3}(100)$-buffered Silicon, where the first $4 \mathrm{~nm}$ of $\mathrm{SrTiO}_{3}$ was deposited on Si by molecular beam epitaxy (MBE) and the subsequent $40 \mathrm{~nm}$ by PLD. The conditions for the substrate preparations are found elsewhere.[78]

The VANs were deposited with an oxygen pressure of $0.2 \mathrm{mbar}$, substrate temperature of $850{ }^{\circ} \mathrm{C}$, and deposition rate of $20 \mathrm{~Hz}$. A $50 \mathrm{~nm} \mathrm{SrRuO} 3$ (SRO) layer was deposited as an intermediate layer.[34,35] The structural quality of the STO layer provided was checked by XRD, depicted in Figure 4-27a, where sharp peaks for STO(001) orientation are visible alongside the Si substrate. The surface was analyzed by AFM (Figure 4-27b) and, while no steps are visible, the surface is extremely smooth, with roughness RMS of $0.21 \mathrm{~nm}$. 


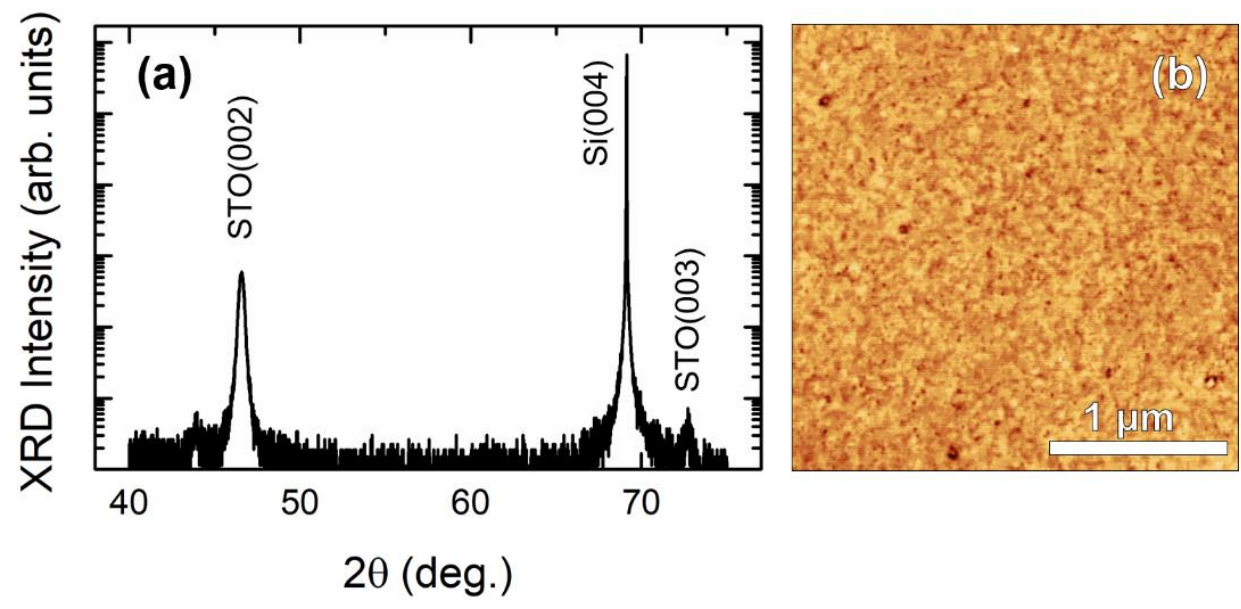

Figure 4-27. (a) XRD pattern of the STO-Buffered Si substrate highlighting the peaks between $40^{\circ}-75^{\circ}$. (b) AFM image of the substrate surface, with roughness RMS of 0.21 nm.

The Energy selective Backscattered SEM (EsB-SEM) analysis top view of the VAN films in Figure 4-28a show the compositional contrast, indicating that the phase separation occurred forming the desired VANs, while the cross-sectional EsB-SEM in Figure 4-28b show all the layers grown and the pillars of LMO formed in the matrix of LLTO. The AFM image given by Figure 4-28c shows the top morphology of the VAN structures grown on a larger scale, with a roughness RMS of $8.8 \mathrm{~nm}$, expected for the conditions used (see Figure 4-11). The average pillar size is $0.015 \pm 0.003 \mu \mathrm{m}^{2}$, in good agreement with previous VAN thin films grown with the same depositions parameters.
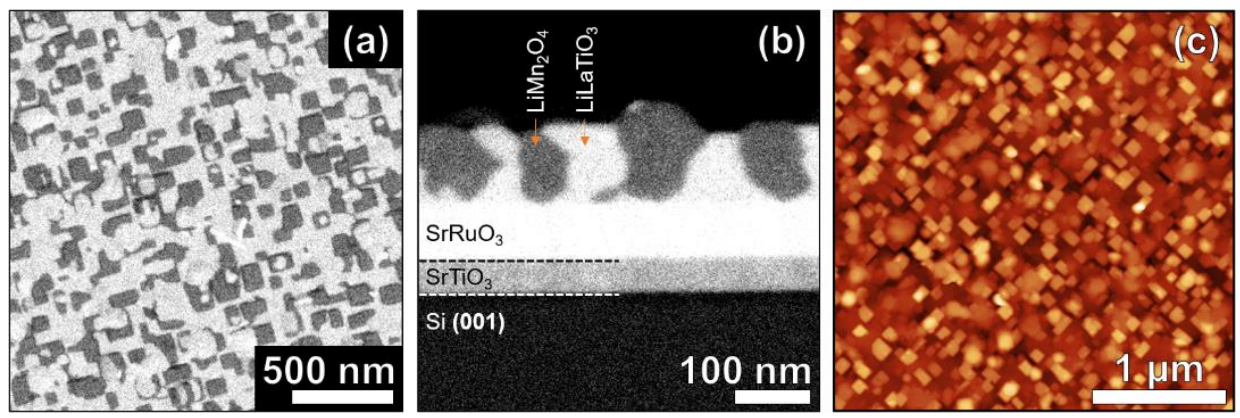

Figure 4-28. Energy selective Backscattered SEM image showing the compositional contrast of the phase separated VANs in (a) Top-view and (b) Cross-section. (c) AFM image of the VAN thin films grown on STO-buffered Silicon with different terminations. 
The VANs on STO-buffered Si were characterized by out-of-plane XRD to evaluate the crystallographic ordering of the involved materials, shown in Figure 4-29. The XRD patterns show all the peaks expected for the LMO and LLTO phases, as well as the peaks for SRO, STO and Si. Li-deficient phases were also observed, namely $\mathrm{Mn}_{2} \mathrm{O}_{3}$ and rutile $\mathrm{TiO}_{2}$. The less crystalline STO layer, when compared to previous depositions, could result in a different strain over the grown materials, resulting in the observed phases. Nonetheless, although more tests are necessary to optimize the growth conditions for the new substrate, this shows the possibility of integration the VAN technology for battery application into Silicon application.
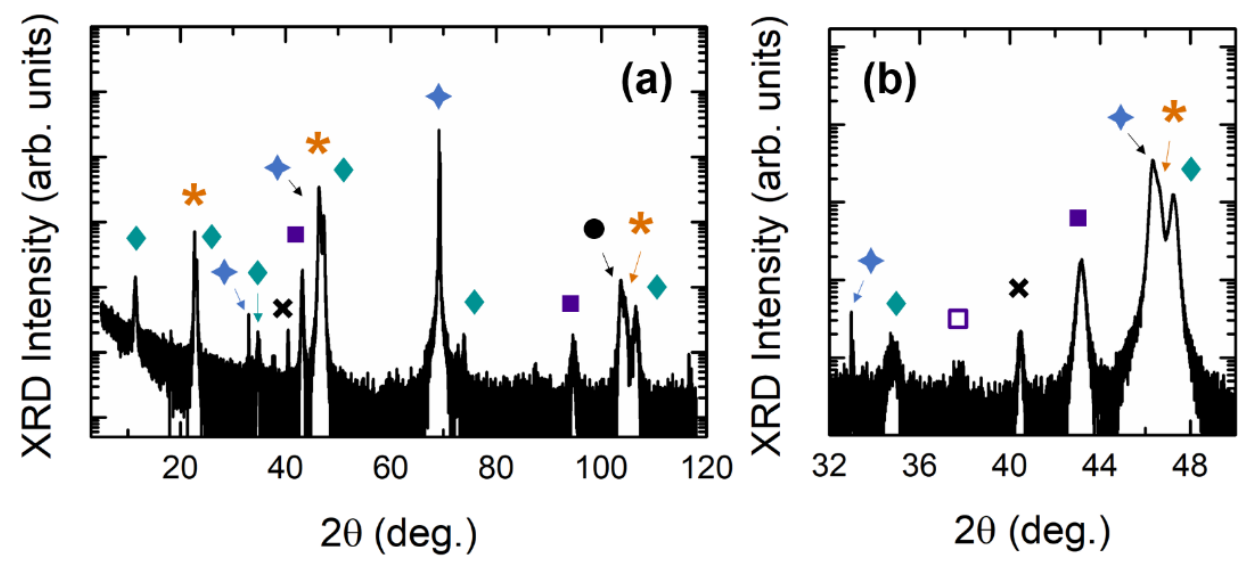

Figure 4-29. (a) Full XRD pattern of the VAN films grown on STO(100)-buffered Silicon substrate, in which LLTO peaks are shown by $\bullet$, LMO by $\boldsymbol{\square}$, SRO by $\boldsymbol{\bullet}$, and the STO peaks by $\star$. The substrate, $\mathrm{Si}(001)$, is indicated by $\bullet$. Impurities phases are indicated by $\square$, corresponding to $\mathrm{Mn}_{2} \mathrm{O}_{3}$, and $\boldsymbol{X}$ to rutile $\mathrm{TiO}_{2}$. (b) XRD pattern highlighting the peaks between $32^{\circ}-50^{\circ}$.

\subsection{Electrochemical analysis}

To evaluate if the grown structures can be used as a three-dimensional battery cathode, the electrochemical behavior was characterized with charge-discharge and cyclic voltammetry $(\mathrm{CV})$ measurements for VANs grown at different conditions. The experiments were performed on the same conditions described in Chapter 2 (section 2.2). $\mathrm{CV}$ was performed at $1 \mathrm{mV} / \mathrm{s}$ between $4.5 \mathrm{~V}$ and $3.3 \mathrm{~V}$, while charge-discharge measurements were done with a current of $1 \mu \mathrm{A}$ to charge to $4.9 \mathrm{~V}$ and discharge to 2.5 $\mathrm{V}$. The test cells were assembled in the same way as described in chapter 2. Specific capacity for LMO was estimated based on the ratio between the cathode and electrolyte (1:3) in the VAN and the VAN layer thickness of $\sim 100 \mathrm{~nm}$. A $50 \mathrm{~nm} \mathrm{SrRuO} 3$ (SRO) layer 
was deposited as an intermediate layer to enhance the electrical transport between the LMTO cathode and the conducting Nb:STO substrate.[35]

The characteristics peaks and plateaus for LMO, described in chapter 2, are not visible in the CV measurements in Figure 4-30. This behavior can be explained by the Ti doping on the LMO pillars, as described in section 4.4.1. The cathode LMO doped with Ti displays better structural stability for the low voltage range ( $3 \mathrm{~V}$ plateau), with the cost of lower capacity. With higher Ti doping levels the capacity drops significantly, making the plateaus also less pronounced.[42,44] The size factor could also play a role, as demonstrated by Okubo et al. for $\mathrm{LiCoO}_{2},[79]$ where the influence of grain size on the capacity drop was studied. For smaller grain sizes, surface effects become more dominant and, therefore, the plateaus would also become less evident.[79] The average lateral size for pillars in the VANs studied here, not considering possible interphases that may occur between LMO and LLTO reducing the active material volume,[80] is $\sim 100 \mathrm{~nm}$, which could affect the electrochemical behavior of these films. In Figure 4-10 (section 4.4.1), a layer of 10 unit cells of insulating LLTO in between the LMO pillars and the current collector is visible, which could hinder the electronic transfer making the LMO pillars electrochemically inactive.
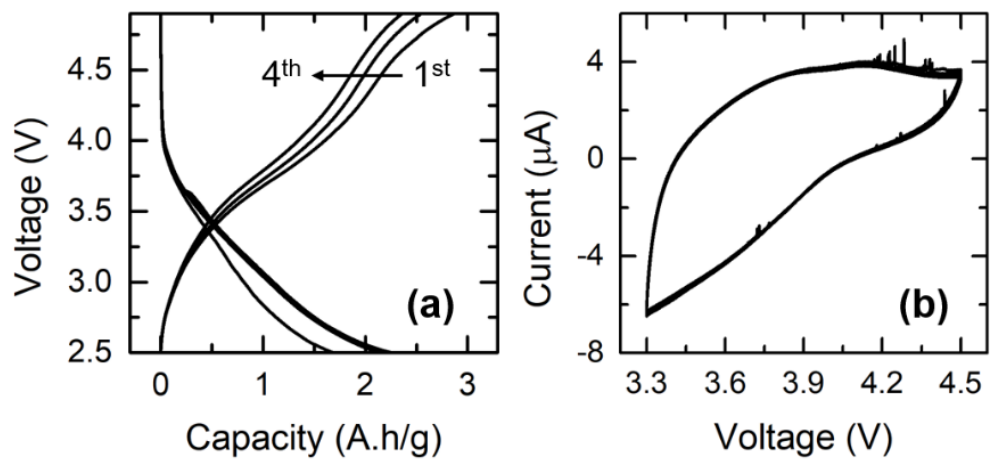

Figure 4-30. (a) Charge-discharge cycles and (b) cyclic voltammetry for the LMO-LLTO VAN grown at $8 \mathrm{~Hz}, 800^{\circ} \mathrm{C}$.

During the charge-discharge, the specific capacity between 4.9 and $2.5 \mathrm{~V}$ of $2 \mathrm{Ah} \cdot \mathrm{g}^{-1}$ $(7.13 \mu \mathrm{Ah})$ is observed, above the expected theoretical bulk capacity for LMO at the analyzed range $\left(\sim 0.25 \mathrm{Ah} \cdot \mathrm{g}^{-1}\right)$. [42,44] In chapter 2 , the influence of surface capacity in thin films was discussed. As mentioned previously, these 3D structures present a surface area approximately 2.5 times higher when compared to 2D thin films, for a thin film with $100 \mathrm{~nm}$ thickness. Considering a surface capacity of $8 \mu \mathrm{Ah} \cdot \mathrm{cm}^{-2}$, observed previously for thin films in chapter 2 , the increased surface area could accommodate another $5 \mu \mathrm{Ah}$. Considering both surface and volumetric capacity, the LMO pillars would be responsible for $82.5 \%(5.9 \mu \mathrm{Ah})$ of the total capacity observed in Figure 4-30. 

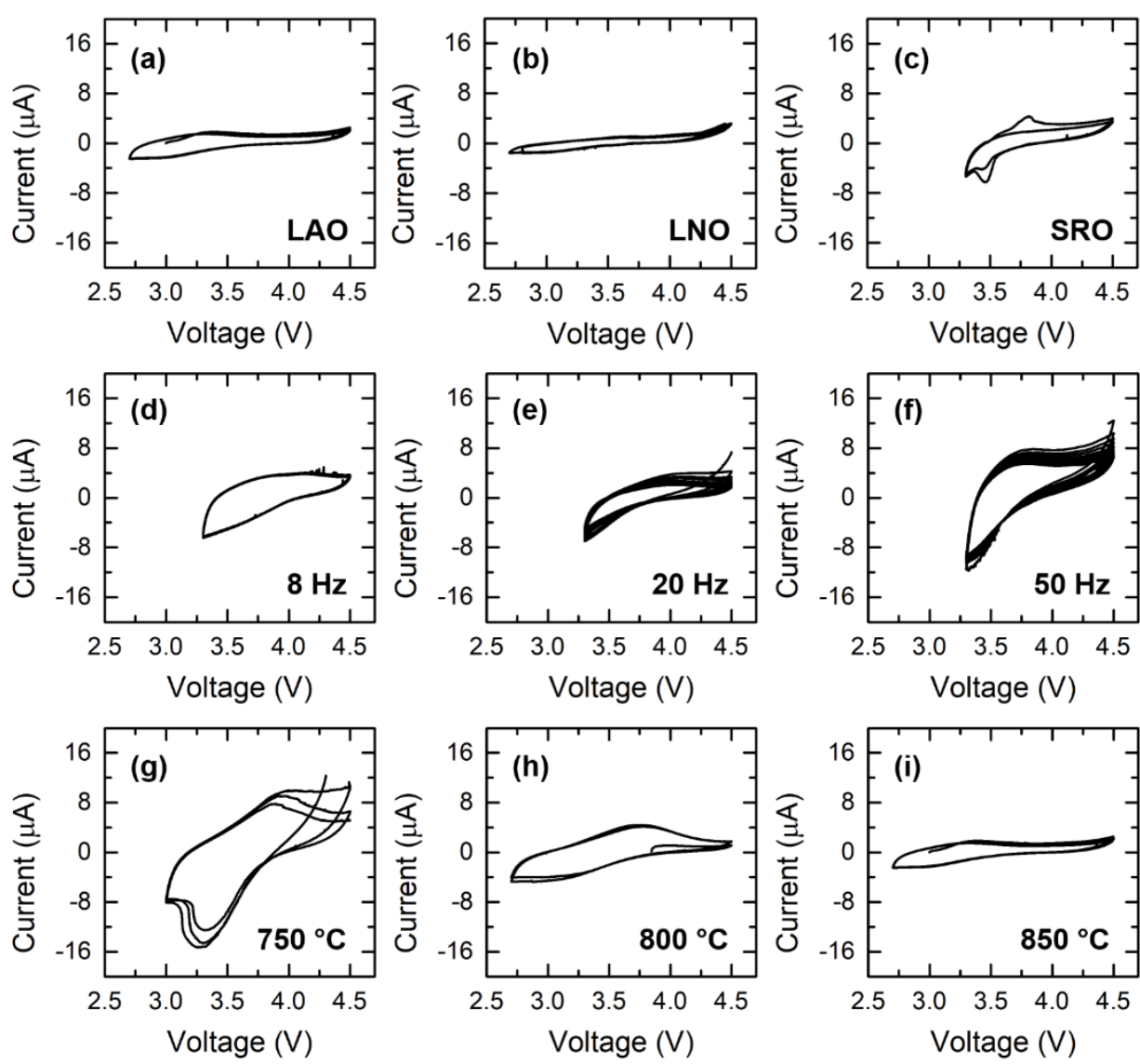

Figure 4-31. Cyclic voltammetry measurements for the LMO-LLTO VAN grown at (ac) $20 \mathrm{~Hz}, 850{ }^{\circ} \mathrm{C}$ for different buffer layers, (d-f) SRO buffer layer, $800{ }^{\circ} \mathrm{C}$ for different deposition rates, and (g-i) LAO buffer layer, $20 \mathrm{~Hz}$ for different growth temperatures.

An explanation for the remaining $17.5 \%$ is that the LLTO matrix is participating in the capacity by intercalating Li into its structure, which was also observed by others in different solid-electrolyte materials.[81] Taking into account the mass of LLTO, the solid electrolyte would be responsible for approximately $158 \mathrm{mAh} \cdot \mathrm{g}^{-1}$ of the total capacity, in agreement with other systems. [81] While the films grown on substrates with different crystal orientation were not measured, VANs deposited at different growth parameters (e.g. temperature, deposition rate, buffer layers) were measured and the cyclic voltammetry and the results are displayed in Figure 4-31.

The CVs displayed in Figure 4-31 show no clear peaks on the positions expected for LMO (4.1 V vs. Li metal), independent of the growth condition, which could be a result of the nanosized structures, as mentioned above.[79] Figure 4-31g-i exhibit a trend of increased CV loop area for lower growth temperatures. Although it is expected higher 
crystallinity for LLTO grown at higher temperatures (see chapter 2), lower currents are observed, indicating higher film resistance. Lower temperatures yield higher number density, increasing the surface capacity as aforementioned. However, a change in number density alone is not sufficient to explain the observed trend, as it would also be visible for different frequencies (as shown in chapter 3). It can be expected that for higher growth temperatures the materials can display a lack of $\mathrm{Li}$, due its volatility, influencing the initial conductivity of the materials and altering the first cycles. Lastly, in section 4.4.1, Figure $4-9$, it is shown that for the temperature range analyzed $\left(750^{\circ} \mathrm{C}\right.$ to $\left.850^{\circ} \mathrm{C}\right)$ the $c / a$ ratio varies from 0.97 to 1 , despite the volume of unit cell being kept nearly constant. This asymmetry could contract/expand the Li diffusion paths, altering the ionic conductivity, but more research is required to support this hypothesis.

The VANs studied in this chapter provide a complex system that, perhaps, the techniques commonly used to measure electrochemistry are not suitable to fully elucidate the ionic and electronic processes. Thus, to elucidate the mechanisms that rule the electrochemistry in these nanocomposites, new ways to measure the functionality at the local level are required, which will be discussed in chapter 5 .

\subsection{Conclusion}

In this chapter, the influence of different growth parameters on the formation of VANs and its properties, namely structure, morphology, and composition, was explored. The trend observed in the temperature dependence study suggested the formation of the Ti doped $\mathrm{LiMn}_{2-\mathrm{y}} \mathrm{Ti}_{\mathrm{y}} \mathrm{O}_{4}$ phase, with $\mathrm{y}=1.5$, instead of the pristine $\mathrm{LMO}$, later confirmed by RSM maps and EELS analysis. The presence of Ti in the LMO pillars could be a result of the high substrate temperature, required for the proper phase separation in the VANs, and the target containing high amounts of Ti which, due the lower formation enthalpy of LMTO, would favor the incorporation of Ti into the pillars. Although the deposition rate can be used to tailor the pillar size of the VANs, here it was shown that it barely affects the composition of the LMO phase. Different substrate orientations can be used to tailor the structure of the matrix and pillar phases and, consequently the morphology and interface between them, where the (110) orientation show great potential with a higher increase in surface area. For the LMO-LLTO combination, the structure was maintained up to $\sim 100 \mathrm{~nm}$, but for thicker films it coalesces into bigger structures, which needs further investigation to mitigate this effect. Different conductive buffer layers were explored as an alternative to the commonly used SRO. Based on experimental results and the simulations presented in chapter 3 , the activation energy for hopping of the adatoms on the substrate surface can be tailored by means of different buffer layers, resulting in A-site and B-site termination. This change in diffusivity could be used to tune the pillar size and increase the pillar-current collector contact. While the hypothesis is correct for some surfaces, the EELS analysis on the LNO buffer layer shows that the intermediate 
LLTO buffer layer is still present. The growth on Si substrate by means of a crystalline buffer layer of STO was shown, indicating the possibility of integration with $\mathrm{Si}$ technology. The expected characteristic peaks were not visible in the electrochemical analysis, due possibility the Ti doping aforementioned and/or the nanosizing of structures, suggesting that the VANs studied in this chapter could provide a complex system beyond the capability of current electrochemical techniques, indicating that new ways to measure the functionality at the device level are required.

\section{Bibliography}

[1] Logvenov, G., Gozar, A., Bozovic, I., High-Temperature Superconductivity in a Single Copper-Oxygen Plane. Science 2009, 326, 699-702.

[2] Bousquet, E., Dawber, M., Stucki, N., Lichtensteiger, C., Hermet, P., Gariglio, S., Triscone, J.-M., Ghosez, P., Improper ferroelectricity in perovskite oxide artificial superlattices. Nature 2008, 452, 732-736.

[3] Yamada, H., Ogawa, Y., Ishii, Y., Sato, H., Kawasaki, M., Akoh, H., Tokura, Y., Engineered Interface of Magnetic Oxides. Science 2004, 305, 646-648.

[4] Ohtomo, A., Hwang, H. Y., A high-mobility electron gas at the $\mathrm{LaAlO}_{3} / \mathrm{SrTiO}_{3}$ heterointerface. Nature 2004, 427, 423-426.

[5] Moshnyaga, V., Damaschke, B., Shapoval, O., Belenchuk, A., Faupel, J., Lebedev, O. I., Verbeeck, J., van Tendeloo, G., Mücksch, M., Tsurkan, V., Tidecks, R., Samwer, K., Structural phase transition at the percolation threshold in epitaxial $(\mathrm{La} 0.7 \mathrm{Ca} 0.3 \mathrm{MnO} 3)_{1-}$ х:(MgO)x nanocomposite films. Nat. Mater. 2003, 2, 247-252.

[6] Zheng, H., Wang, J., Lofland, S. E., Ma, Z., Mohaddes-Ardabili, L., Zhao, T., Salamanca-Riba, L., Shinde, S. R., Ogale, S. B., Bai, F., Viehland, D., Jia, Y., Schlom, D. G., Wuttig, M., Roytburd, A., Ramesh, R., Multiferroic $\mathrm{BaTiO}_{3}-\mathrm{CoFe}_{2} \mathrm{O}_{4}$ Nanostructures. Science 2004, 303, 661-663.

[7] Matthews, J. W., Mader, S., Light, T. B., Accommodation of Misfit Across the Interface Between Crystals of Semiconducting Elements or Compounds. J. Appl. Phys. 1970, 41, 3800 .

[8] Macmanus-Driscoll, J. L., Self-assembled heteroepitaxial oxide nanocomposite thin film structures: Designing interface-induced functionality in electronic materials. Adv. Funct. Mater. 2010, 20, 2035-2045.

[9] Zheng, H., Straub, F., Zhan, Q., Yang, P. L., Hsieh, W. K., Zavaliche, F., Chu, Y. H., Dahmen, U., Ramesh, R., Self-assembled growth of $\mathrm{BiFeO}_{3}-\mathrm{CoFe}_{2} \mathrm{O}_{4}$ nanostructures. Adv. Mater. 2006, 18, 2747-2752.

[10] Zheng, H., Zhan, Q., Zavaliche, F., Sherburne, M., Straub, F., Cruz, M. P., Chen, L. Q., Dahmen, U., Ramesh, R., Controlling self-assembled perovskite-spinel nanostructures. Nano Lett. 2006, 6, 1401-1407.

[11] Zheng, H., Wang, J., Mohaddes-Ardabili, L., Wuttig, M., Salamanca-Riba, L., Schlom, 
D. G., Ramesh, R., Three-dimensional heteroepitaxy in self-assembled $\mathrm{BaTiO}_{3}-\mathrm{CoFe}_{2} \mathrm{O}_{4}$ nanostructures. Appl. Phys. Lett. 2004, 85, 2035-2037.

[12] Yan, L., Yang, Y., Wang, Z., Xing, Z., Li, J., Viehland, D., Review of magnetoelectric perovskite-spinel self-assembled nano-composite thin films. J. Mater. Sci. 2009, 44, $5080-5094$.

[13] Roshko, A., Harvey, T. E., Hyland, B. L., Lehman, S. Y., Cobry, K. D., Lateral variations in self-assembled InGaAs quantum dot distributions. J. Cryst. Growth 2009, 311, 4109-4115.

[14] Stratulat, S. M., Lu, X., Morelli, A., Hesse, D., Erfurth, W., Alexe, M., Nucleationinduced self-assembly of multiferroic $\mathrm{BiFeO}_{3}-\mathrm{CoFe}_{2} \mathrm{O}_{4}$ nanocomposites. Nano Lett. 2013, 13, 3884-3889.

[15] Harrington, S. A., Durrell, J. H., Wang, H., Wimbush, S. C., Tsai, C. F., MacManusDriscoll, J. L., Understanding nanoparticle self-assembly for a strong improvement in functionality in thin film nanocomposites. Nanotechnology 2010, 21, 95604.

[16] Gutiérrez, J., Llordés, A., Gázquez, J., Gibert, M., Romà, N., Ricart, S., Pomar, A., Sandiumenge, F., Mestres, N., Puig, T., Obradors, X., Strong isotropic flux pinning in solution-derived $\mathrm{YBa}_{2} \mathrm{Cu}_{3} \mathrm{O}_{7-\mathrm{x}}$ nanocomposite superconductor films. Nat. Mater. 2007, $6,367-373$.

[17] Wu, W., Schulman, J. N., Hsu, T. Y., Efron, U., Effect of size nonuniformity on the absorption spectrum of a semiconductor quantum dot system. Appl. Phys. Lett. 1987, 51, $710-712$.

[18] Solomon, G. S., Competing processes in the surface ordering of InAs islands using a subsurface island superlattice. Appl. Phys. Lett. 2004, 84, 2073-2075.

[19] Goyal, A., Kang, S., Leonard, K. J., Martin, P. M., Gapud, A. A., Varela, M., Paranthaman, M., Ijaduola, A. O., Specht, E. D., Thompson, J. R., Christen, D. K., Pennycook, S. J., List, F. A., Irradiation-free, columnar defects comprised of selfassembled nanodots and nanorods resulting in strongly enhanced flux-pinning in $\mathrm{YBa}$ ${ }_{2} \mathrm{Cu}_{3} \mathrm{O}_{7-\delta}$ films. Supercond. Sci. Technol. 2005, 18, 1533-1538.

[20] Putnis, A., An Introduction to Mineral Sciences. Cambridge University Press 1992.

[21] Kuroda, S., Nishizawa, N., Takita, K., Mitome, M., Bando, Y., Osuch, K., Dietl, T., Origin and control of high-temperature ferromagnetism in semiconductors. Nat. Mater. 2007, 6, 440-446.

[22] Wójcik, A., Godlewski, M., Guziewicz, E., Kopalko, K., Jakieła, R., Kiecana, M., Sawicki, M., Guziewicz, M., Putkonen, M., Niinistö, L., Dumont, Y., Keller, N., Low temperature growth of $\mathrm{ZnMnO}$ : A way to avoid inclusions of foreign phases and spinodal decomposition. Appl. Phys. Lett. 2007, 90, 82502.

[23] Kim, H.-S., Bi, L., Paik, H., Yang, D.-J., Park, Y. C., Dionne, G. F., Ross, C. A., SelfAssembled Single-Phase Perovskite Nanocomposite Thin Films. Nano Lett. 2010, 10, 597-602.

[24] Park, S., Horibe, Y., Asada, T., Wielunski, L. S., Lee, N., Bonanno, P. L., O’Malley, S. M., Sirenko, A. A., Kazimirov, A., Tanimura, M., Gustafsson, T., Cheong, S.-W., Highly Aligned Epitaxial Nanorods with a Checkerboard Pattern in Oxide Films. Nano Lett. 2008, 8, 720-724. 
[25] O’Malley, S. M., Bonanno, P. L., Ahn, K. H., Sirenko, A. A., Kazimirov, A., Tanimura, M., Asada, T., Park, S., Horibe, Y., Cheong, S.-W., Epitaxial checkerboard arrangement of nanorods in $\mathrm{ZnMnGaO}_{4}$ films studied by x-ray diffraction. Phys. Rev. B 2008, 78, 165424.

[26] Bouar, Y. Le, Loiseau, A., Khachaturyan, A. G., Origin of chessboard-like structures in decomposing alloys. Theoretical model and computer simulation. Acta Mater. 1998, 46, $2777-2788$.

[27] Zhang, B., Lelovic, M., Soffa, W. A., The formation of polytwinned structures in $\mathrm{Fe} \square \mathrm{Pt}$ and $\mathrm{Fe} \square \mathrm{Pd}$ alloys. Scr. Metall. Mater. 1991, 25, 1577-1582.

[28] Herger, R., Willmott, P. R., Bunk, O., Schlepütz, C. M., Patterson, B. D., Delley, B., Shneerson, V. L., Lyman, P. F., Saldin, D. K., Surface structure of $\mathrm{SrTiO}_{3}(001)$. Phys. Rev. B - Condens. Matter Mater. Phys. 2007, 76, 195435.

[29] Chrisey, D. B., Hubler, G. K., Pulsed Laser Deposition of Thin Films. John Wiley \& Sons, New York 1994.

[30] Lowndes, D. H., Geohegan, D. B., Puretzky, A. A., Norton, D. P., Rouleau, C. M., Synthesis of Novel Thin-Film Materials by Pulsed Laser Deposition. Science 1996, 273, 898 LP - 903.

[31] Tyunina, M., Levoska, J., Leppävuori, S., Experimental studies and modeling of $\mathrm{Pb}-\mathrm{Zr}-$ Ti-O film growth in pulsed laser deposition. J. Appl. Phys. 1998, 83, 5489-5496.

[32] Dam, B., Stauble-Pumpin, B., Growth mode issues in epitaxy of complex oxide thin films. J. Mater. Sci. Mater. Electron. 1998, 9, 217-226.

[33] Blank, D. H. A., Rijnders, G. J. H. M., Koster, G., Rogalla, H., In-situ monitoring by reflective high energy electron diffraction during pulsed laser deposition. Appl. Surf. Sci. 1999, 138-139, 17-23.

[34] Hendriks, R., Cunha, D. M., Singh, D. P., Huijben, M., Enhanced Lithium Transport by Control of Crystal Orientation in Spinel $\mathrm{LiMn}_{2} \mathrm{O}_{4}$ Thin Film Cathodes. ACS Appl. Energy Mater. 2018, 1, 7046-7051.

[35] Suzuki, K., Kim, K., Taminato, S., Hirayama, M., Kanno, R., Fabrication and electrochemical properties of $\mathrm{LiMn}_{2} \mathrm{O}_{4} / \mathrm{SrRuO}_{3}$ multi-layer epitaxial thin film electrodes. J. Power Sources 2013, 226, 340-345.

[36] Cunha, D. M., Vos, C. M., Hendriks, T. A., Singh, D. P., Huijben, M., Morphology Evolution during Lithium-Based Vertically Aligned Nanocomposite Growth. ACS Appl. Mater. Interfaces 2019, 11, 44444-44450.

[37] Stramare, S., Thangadurai, V., Weppner, W., Lithium Lanthanum Titanates: A Review. Chem. Mater. 2003, 34, 3974-3990.

[38] Ohnishi, T., Mitsuishi, K., Nishio, K., Takada, K., Epitaxy of $\mathrm{Li}_{3 x} \mathrm{La}_{2 / 3-x} \mathrm{TiO}_{3}$ films and the influence of la ordering on Li-ion conduction. Chem. Mater. 2015, 27, 1233-1241.

[39] Mishra, S. K., Ceder, G., Structural stability of lithium manganese oxides. Phys. Rev. B Condens. Matter Mater. Phys. 1999, 59, 6120-6130.

[40] Morcrette, M., Barboux, P., Perrière, J., Brousse, T., Traverse, A., Boilot, J. P., Nonstoichiometry in $\mathrm{LiMn}_{2} \mathrm{O}_{4}$ thin films by laser ablation. Solid State Ionics 2001, 138, $213-$ 
219.

[41] Zhang, Y., Hughes, R. A., Britten, J. F., Gong, W., Preston, J. S., Botton, G. A., Niewczas, M., Epitaxial Ni-Mn-Ga films derived through high temperature in situ depositions. Smart Mater. Struct. 2009, 18, 025019.

[42] He, G., Li, Y., Li, J., Yang, Y., Spinel $\mathrm{LiMn}_{2-\mathrm{x}} \mathrm{Ti}_{\mathrm{x}} \mathrm{O}_{4}(\mathrm{x}=0.5,0.8)$ with High capacity and enhanced cycling stability synthesized by a modified sol-gel method. Electrochem. Solid-State Lett. 2010, 13, 19-22.

[43] Pigliapochi, R., Seymour, I. D., Merlet, C., Pell, A. J., Murphy, D. T., Schmid, S., Grey, C. P., Structural Characterization of the Li-Ion Battery Cathode Materials $\mathrm{LiTi}_{\mathrm{x}} \mathrm{Mn}_{2-\mathrm{x}} \mathrm{O} 4$ $(0.2 \leq \mathrm{x} \leq 1.5)$ : A Combined Experimental 7Li NMR and First-Principles Study. Chem. Mater. 2018, 30, 817-829.

[44] Wang, S., Yang, J., Wu, X., Li, Y., Gong, Z., Wen, W., Lin, M., Yang, J., Yang, Y., Toward high capacity and stable manganese-spinel electrode materials: A case study of Ti-substituted system. J. Power Sources 2014, 245, 570-578.

[45] Petrov, K., Rojas, R. M., Alonso, P. J., Amarilla, J. M., Lazarraga, M. G., Rojo, J. M., Cation distribution and phase transformations in $\mathrm{LiMn}_{2-\mathrm{y}} \mathrm{Ti}_{\mathrm{y}} \mathrm{O}_{4}(0.2 \leq \mathrm{y} \leq 1.5)$ solid solutions. Solid State Sci. 2005, 7, 277-286.

[46] Ettlinger, R. B., Cazzaniga, A., Canulescu, S., Pryds, N., Schou, J., Pulsed laser deposition from $\mathrm{ZnS}$ and $\mathrm{Cu}_{2} \mathrm{SnS}_{3}$ multicomponent targets. Appl. Surf. Sci. 2015, 336, $385-390$.

[47] Develos, K. D., Kusunoki, M., Mukaida, M., Ohshima, S., Effect of deposition rate on the surface morphology of $\mathrm{CeO}_{2}$ films deposited by pulsed laser deposition. Phys. $C$ Supercond. its Appl. 1999, 320, 21-30.

[48] Sun, T., Ma, J., Yan, Q. Y., Huang, Y. Z., Wang, J. L., Hng, H. H., Influence of pulsed laser deposition rate on the microstructure and thermoelectric properties of $\mathrm{Ca}_{3} \mathrm{Co}_{4} \mathrm{O}_{9}$ thin films. J. Cryst. Growth 2009, 311, 4123-4128.

[49] Kim, J.-W., Kang, H.-S., Lee, S.-Y., Effect of Deposition Rate on the Property of ZnO Thin Films Deposited by Pulsed Laser Deposition. J. Electr. Eng. Technol. 2006, 1, 98 100 .

[50] Sano, T., Kim, C.-S., Rohrer, G. S., Shape Evolution of $\mathrm{SrTiO}_{3}$ Crystals During Coarsening in a Titania-Rich Liquid. J. Am. Ceram. Soc. 2005, 88, 993-996.

[51] Padilla, J., Vanderbilt, D., Ab initio study of $\mathrm{BaTiO}_{3}$ surfaces. Phys. Rev. B 1997, 56, $1625-1631$.

[52] Meyer, B., Padilla, J., Vanderbilt, D., Theory of $\mathrm{PbTiO}_{3}, \mathrm{BaTiO}_{3}$, and $\mathrm{SrTiO}_{3}$ surfaces. Faraday Discuss. 1999, 114, 395-405.

[53] Cunha, D. M., Hendriks, T. A., Vasileiadis, A., Vos, C. M., Verhallen, T., Singh, D. P., Wagemaker, M., Huijben, M., Doubling Reversible Capacities in Epitaxial $\mathrm{Li}_{4} \mathrm{Ti}_{5} \mathrm{O}_{12}$ Thin Film Anodes for Microbatteries. ACS Appl. Energy Mater. 2019, 2, 3410-3418.

[54] Mishra, R. K., Thomas, G., Surface energy of spinel. J. Appl. Phys. 1977, 48, 45764580 .

[55] Huang, M.-R., Lin, C.-W., Lu, H.-Y., Crystallographic facetting in solid-state reacted 
$\mathrm{LiMn}_{2} \mathrm{O}_{4}$ spinel powder. Appl. Surf. Sci. 2001, 177, 103-113.

[56] Van Der Laag, N. J., Fang, C. M., De With, G., De Wijs, G. A., Brongersma, H. H., Geometry of $\{001\}$ Surfaces of Spinel $\left(\mathrm{MgAl}_{2} \mathrm{O}_{4}\right)$ : First-Principles Simulations and Experimental Measurements. J. Am. Ceram. Soc. 2005, 88, 1544-1548.

[57] Tan, Z., Slutsker, J., Roytburd, A. L., Epitaxial self-assembly of multiferroic nanostructures. J. Appl. Phys. 2009, 105, DOI: 10.1063/1.3056160.

[58] Ohta, H., Mizoguchi, T., Aoki, N., Yamamoto, T., Sabarudin, A., Umemura, T., Lithium-ion conducting $\mathrm{La}_{2 / 3-x} \mathrm{Li}_{3 \times} \mathrm{TiO}_{3}$ solid electrolyte thin films with stepped and terraced surfaces. Appl. Phys. Lett. 2012, 100, 173107.

[59] Huang, J., MacManus-Driscoll, J. L., Wang, H., New epitaxy paradigm in epitaxial selfassembled oxide vertically aligned nanocomposite thin films. J. Mater. Res. 2017, 32, 4054-4066.

[60] Pimpinelli, A., Villain, J., Physics of Crystal Growth. Cambridge University Press 1998.

[61] MacManus-Driscoll, J. L., Zerrer, P., Wang, H., Yang, H., Yoon, J., Fouchet, A., Yu, R., Blamire, M. G., Jia, Q., Strain control and spontaneous phase ordering in vertical nanocomposite heteroepitaxial thin films. Nat. Mater. 2008, 7, 314-320.

[62] Long, J. W., Dunn, B., Rolison, D. R., White, H. S., Three-dimensional battery architectures. Chem. Rev. 2004, 104, 4463-4492.

[63] Oudenhoven, J. F. M., Baggetto, L., Notten, P. H. L., All-solid-state lithium-ion microbatteries: A review of various three-dimensional concepts. Adv. Energy Mater. $2011,1,10-33$.

[64] Yue, C., Li, J., Lin, L., Fabrication of Si-based three-dimensional microbatteries: A review. Front. Mech. Eng. 2017, 12, 459-476.

[65] Amos, C. D., Roldan, M. A., Varela, M., Goodenough, J. B., Ferreira, P. J., Revealing

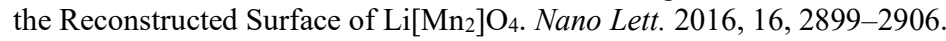

[66] Solmaz, A., Huijben, M., Koster, G., Egoavil, R., Gauquelin, N., Van Tendeloo, G., Verbeeck, J., Noheda, B., Rijnders, G., Domain Selectivity in $\mathrm{BiFeO}_{3}$ Thin Films by Modified Substrate Termination. Adv. Funct. Mater. 2016, 26, 2882-2889.

[67] Woo, S., Jeong, H., Lee, S. A., Seo, H., Lacotte, M., David, A., Kim, H. Y., Prellier, W., Kim, Y., Choi, W. S., Surface properties of atomically flat poly-crystalline $\mathrm{SrTiO}_{3}$. Sci. Rep. 2015, 5, 1-7.

[68] Kuiper, B., Self-assembled $\mathrm{SrRuO}_{3}$ nanowires through selective growth on $\mathrm{DyScO}_{3}$ surface terminations. 2009.

[69] Chen, X. Y., Wong, K. H., Mak, C. L., Liu, J. M., Yin, X. B., Wang, M., Liu, Z. G., The orientation-selective growth of $\mathrm{LaNiO}_{3}$ films on $\mathrm{Si}(100)$ by pulsed laser deposition using a MgO buffer. Appl. Phys. A Mater. Sci. Process. 2002, 75, 545-549.

[70] Murtaza, G., Ahmad, I., Shift of indirect to direct bandgap and optical response of $\mathrm{LaAlO}_{3}$ under pressure. J. Appl. Phys. 2012, 111, 123116.

[71] Rakshit, S., Gopalakrishnan, P. S., Oxygen nonstoichiometry and its effect on the structure of $\mathrm{LaNiO}_{3}$. J. Solid State Chem. 1994, 110, 28-31. 
[72] Golalikhani, M., Lei, Q. Y., Chen, G., Spanier, J. E., Ghassemi, H., Johnson, C. L., Taheri, M. L., Xi, X. X., Stoichiometry of $\mathrm{LaAlO}_{3}$ films grown on $\mathrm{SrTiO}_{3}$ by pulsed laser deposition. J. Appl. Phys. 2013, 114, 1-6.

[73] Fowlie, J., Gibert, M., Tieri, G., Gloter, A., Íñiguez, J., Filippetti, A., Catalano, S., Gariglio, S., Schober, A., Guennou, M., Kreisel, J., Stéphan, O., Triscone, J. M., Conductivity and Local Structure of $\mathrm{LaNiO}_{3}$ Thin Films. Adv. Mater. 2017, 29, 1-5.

[74] Koster, G., Klein, L., Siemons, W., Rijnders, G., Dodge, J. S., Eom, C. B., Blank, D. H. A., Beasley, M. R., Structure, physical properties, and applications of $\mathrm{SrRuO}_{3}$ thin films. Rev. Mod. Phys. 2012, 84, 253-298.

[75] Huijben, M., Brinkman, A., Koster, G., Rijnders, G., Hilgenkamp, H., Blank, D. H. A., Structure-Property Relation of $\mathrm{SrTiO}_{3} / \mathrm{LaAlO}_{3}$ Interfaces. Adv. Mater. 2009, 21, 16651677.

[76] Shu, J., Yi, T. F., Shui, M., Wang, Y., Zhu, R. S., Chu, X. F., Huang, F., Xu, D., Hou, L., Comparison of electronic property and structural stability of $\mathrm{LiMn}_{2} \mathrm{O}_{4}$ and

$\mathrm{LiNi}_{0.5} \mathrm{Mn}_{1.5} \mathrm{O}_{4}$ as cathode materials for lithium-ion batteries. Comput. Mater. Sci. 2010, $50,776-779$.

[77] Narayanan, V., Frank, M. M., Demkov, A. A., Thin Films on Silicon: Electronic and Photonic Applications. World Scientific Publishing 2016.

[78] Marchiori, C., Sousa, M., Guiller, A., Siegwart, H., Locquet, J.-P., Fompeyrine, J., Norga, G. J., Seo, J. W., Thermal stability of the $\mathrm{SrTiO}_{3}(\mathrm{Ba}, \mathrm{Sr}) \mathrm{O}$ stacks epitaxially grown on Si. Appl. Phys. Lett. 2006, 88, 72913.

[79] Okubo, M., Hosono, E., Kim, J., Enomoto, M., Kojima, N., Kudo, T., Zhou, H., Honma, I., Nanosize effect on high-rate Li-ion intercalation in $\mathrm{LiCoO}_{2}$ electrode. J. Am. Chem. Soc. 2007, 129, 7444-7452.

[80] Kim, S., Hirayama, M., Suzuki, K., Kanno, R., Hetero-epitaxial growth of $\mathrm{Li} 0.17 \mathrm{La} 0.61 \mathrm{TiO}_{3}$ solid electrolyte on $\mathrm{LiMn}_{2} \mathrm{O}_{4}$ electrode for all solid-state batteries. Solid State Ionics 2014, 262, 578-581.

[81] Schwietert, T. K., Arszelewska, V. A., Wang, C., Yu, C., Vasileiadis, A., de Klerk, N. J. J., Hageman, J., Hupfer, T., Kerkamm, I., Xu, Y., van der Maas, E., Kelder, E. M., Ganapathy, S., Wagemaker, M., Clarifying the relationship between redox activity and electrochemical stability in solid electrolytes. Nat. Mater. 2020, 19, 429-435. 


\title{
Chapter 5
}

\section{Electrochemistry at the Nanoscale}

\begin{abstract}
Solid-state microbatteries can facilitate miniaturization, create more flexibility for the design of stand-alone microelectronic devices and enhance their applicability. However, the successful application of all-solid-state microbatteries depends strongly on the improvement of energy density and lifetime. Therefore, perfect control on the interfacial properties between the electrodes and electrolyte is needed but remains a great challenge. SPM techniques allow the measurement of the electrochemistry taking place on the nanoscale, which can be used to elucidate structure/function relationships in battery materials with exceptional resolution. To achieve insight into the non-uniform distribution of lithium activity at the cathode-electrolyte interface, First Order Reversal Curve current-voltage (FORC-IV) analysis is applied. This technique enables the probing of nanoscale electrochemistry due to the capability to measure local ionic currents. In FORC-IV measurements, current through the sample is measured with an AFM probe in contact mode as a function of the bias waveform, a triangular wave with increasing amplitude, allowing the measurement of a variety of electrochemical processes. Depending on the rate and reversibility of these processes, this will result in hysteretic IV curves, proportional to the voltage-activated electrochemical processes and transport at a given spot. In this chapter, the local lithium diffusion in $\mathrm{LiMn}_{2} \mathrm{O}_{4}$ and $\mathrm{VAN}$ thin films, epitaxially grown by Pulsed Laser Deposition on single crystalline $\mathrm{Nb}$-doped $\mathrm{SrTiO}_{3}$ substrates, was studied. By changing the crystal orientation of the underlying single crystalline substrate, the specific orientation of the $\mathrm{LiMn}_{2} \mathrm{O}_{4}$ thin film can be controlled and, therefore, the cathode surface towards the electrolyte. The FORC results expose the electrochemical activity for different crystal facets indicating the topography dependent lithium diffusion. These results provide better understanding of the importance of the specific crystal facet in contact with the adjacent electrolyte to enable enhanced engineering of the interfacial properties.
\end{abstract}




\subsection{Introduction}

Based on diffusion in a solid versus a liquid, the success of solid-state batteries depend on the capability to address the nanoscale ionic processes in the thin films, at their interfaces, and the combined electronic-ionic transport. As described in chapter 4, at submicrometer thicknesses, the nanoionic properties of the thin film system become more dominant and the interfaces between layers represent a higher volume fraction in the final cell when compared to bulk. These conditions create challenges for the physical characterization of battery materials such as the local correlation between a nanoscale stimulus and the resulting material response.[1,2]

The ability to image function on the nanoscale can be used to elucidate the relationship between crystal structure and functionality in nanostructured materials, often on working devices, and with exceptional resolution. The development of scanning probe microscopy (SPM)[1] techniques, namely electrochemical strain microscopy (ESM) $[3,4]$ and first order reversal curve current-voltage (FORC-IV)[5-8], has enabled the probing of nanoscale electrochemistry due to the capability to measure local ionic currents, which provides crucial insight into the local electrochemical reactions and ionic flows.

ESM is based on applying an electric bias to an AFM tip in contact mode, which will induce interfacial electrochemical reactions and ionic motion in the material, caused by the concentrated electric field in the small volume of material below the tip. The strong coupling between ionic concentration and molar volume results in localized strains and hence minute deformations of the solid, which can be detected by the cantilever. However, the signal requires advanced processing as many contributions might be present, e.g. piezoelectric, and charge injection.[5,9,10] On the other hand, FORC-IV overcomes this shortcoming, where the small changes in ionic state are detectable via the variations in electronic transport, as it will be further explained in section 5.2.

Since the cathode materials of interest are not perfect ionic conductors, which is a requirement for ESM, here, the FORC-IV technique will be applied to provide detailed information about the lithium diffusion mechanism at a resolution of tens of nanometers, showing variations in ionic conductivity for different crystal facets. To deconvolute the transport and electrochemical reactivity, the measurements at each location will be performed as a function of bias sweep amplitude using a specified waveform. A set of hysteresis loops will be obtained as a function of excitation window at each location with high spatial correlation, eliminating the need for spatial alignment of maps obtained sequentially. This enables the detailed characterization of voltage-activated lithium transport at a certain critical voltage. Therefore, this technique offers the capability to distinguish local regions with low effective mobility of lithium ions from other regions with high lithium mobility. 
In this chapter, to study the nanoscale characteristics of solid-state materials FORC-IV is applied. As model system, $\mathrm{LiMn}_{2} \mathrm{O}_{4}$ (LMO) as cathode material [11,12] deposited by PLD on crystalline substrates, as well as polycrystalline Platinum, is analyzed. In addition, an analysis is performed on VAN thin films grown on STO(100) buffered Silicon, to elucidate the mechanisms present in these structures.

\subsection{First Order Reversal Curve Current-Voltage}

The ESM detection of electrochemical processes is based on the electromechanical response of a system and can provide ambiguous results $[5,9,10]$ requiring careful separation of piezoelectric, flexoelectric, Vegard strain, Faradaic, charge injection, chemical dipole, and other effects, all of which can contribute to the ESM signal. Thus, ESM is only applicable to pure ionic conductors, where the current is Faradaic. In order to overcome these shortcomings and probe the electrochemistry in solid mixed ionicelectronic conductors (MIECs), FORC-IV technique was introduced.[5-8] In such systems, the electronic conductivity is inherently dependent on the ionic transport, as ions act as mobile dopants changing the local conductivity as they migrate through the material under the external electric field. Thus, even small changes in the ionic state of the system are amplified into detectable variations in the electronic transport.

(a)

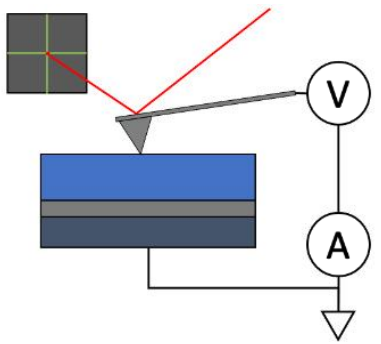

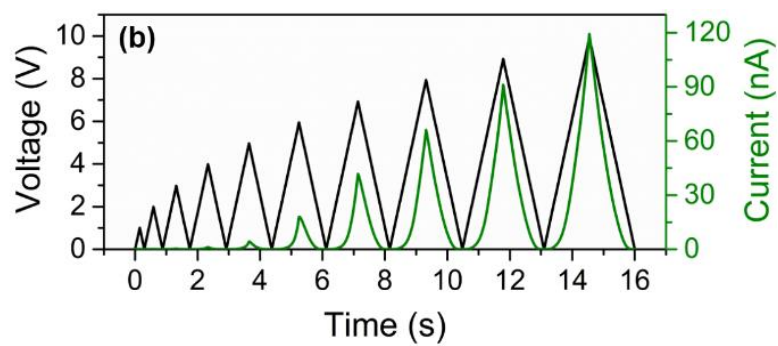

Figure 5-1. (a) FORC-IV schematic, highlighting the AFM tip in contact mode where a bias is applied, and the bottom electrode is grounded. The setup resembles that of a conductive AFM measurement, except by (b) the voltage waveform consisting of several triangular pulses with increasing amplitude, and the respective current recorded.

A good example of such behavior is the LTO system where the reversible cycling between $\mathrm{Li}_{4} \mathrm{Ti}_{5} \mathrm{O}_{12}$ and $\mathrm{Li}_{7} \mathrm{Ti}_{5} \mathrm{O}_{12}$ results in only $0.2 \%$ volumetric change, which would be undetectable by ESM.[13] The two materials are respectively electronically insulating and conductive, where a simple current mapping would reveal the spatial distribution of the two phases at different charging states of the battery.[13] An investigation of more complex systems, where both the reactant and product are MIECs or the insulatorconductor transition proceeds gradually, requires the use of the FORC-IV, as depicted in 
Figure 5-1a. A voltage waveform consisting of several triangular pulses with increasing amplitude $\left(\mathrm{V}_{\mathrm{p}}\right)$, is applied to a conductive AFM tip in contact mode, and the current is recorded, as in Figure 5-1b. The IV curves will be non-hysteretic unless the circuit includes a large capacitance, Joule heating occurs, or an electrochemical process changes the conductivity. If the two former effects are considered and cared for (section 5.2.1),[5] the FORC-IV hysteresis loop area will serve as a measure of the electrochemical activity of the sample beneath the tip. When the FORC-IV loop area is plotted against the peak bias, an exponentially increasing curve is formed. Below the threshold voltage for activation of an electrochemical process (e.g. ion migration, Faradaic process) the FORCIV loop area will be negligible, and above the critical peak bias it will rapidly increase.

The potential of FORC-IV technique is revealed in the spectroscopic mode, when the IV hysteresis is measured consecutively at each location of a spatial grid. A good example is the widely studied $\mathrm{BiFeO}_{3}-\mathrm{CoFe}_{2} \mathrm{O}_{4}(\mathrm{BFO}-\mathrm{CFO})$ nanocomposite mentioned in chapter 4.[7] The interface being inherently more conductive than the matrix or CFO when no electrochemical processes are active, and the voltage-controlled on-off switching of the conductivity on the interface suggests the possible process of oxygen vacancies that accumulate at the grain boundary. However, the FORC-IV loop area maps for this system clearly show that the electrochemical activity concentrates at the interfaces and is activated in different locations and at different voltages.[7]

\subsubsection{Parasitic Capacitance}

For scanning capacitance microscopy ( $\mathrm{SCM})$, the effect of parasitic capacitance $\left(\mathrm{C}_{\mathrm{par}}\right)$ has been extensively investigated. However, for conductive AFM (cAFM) methods, like FORC-IV, this effect is rarely mentioned.[14] From SCM studies, it is known that $\mathrm{C}_{\text {par }}$ is in the $\mathrm{pF}$ range, $[15,16]$ thus, when cAFM-IV curves are performed by applying a voltage sweep with a sweep rate (sr) dV/dt, $\mathrm{C}_{\mathrm{par}}$ should contribute significantly to the sensitivity limit of cAFM measurements. This effect influences the IV measurements because the variation in electric field $(\mathrm{sr} \neq 0)$ results in tip-sample capacitance that alters the distance between the tip and the sample. This small change in distance generates displacement currents $\left(\mathrm{I}_{\text {disp }}\right)$, which are about equal to or exceed the current of interest $\left(\mathrm{I}_{\text {int }}\right)$. Slower scan rates may reduce this problem, but lead to increased electrical stress.[14]

As mentioned, local IV measurements are typically performed applying a voltage sweep with a voltage sweep rate $s r$, and a forward and a backward sweep are performed within one measurement sequence where for both directions different $s r$ can be defined. Considering the same $s r$ for both directions, only the sign of $s r$ changes for forward (positive) and backward (negative). Then, the overall current ( $\left.\mathrm{I}_{\text {total }}\right)$ is composed (Equation (5.1) of the $\mathrm{I}_{\text {int }}, \mathrm{I}_{\mathrm{disp}}$ in case of any effective parasitic capacitance $\mathrm{C}_{\text {par }}$ according 
to Equation (5.2), the offset current $\left(\mathrm{I}_{\text {off }}\right)$ due to the measurement setup, and transient effects ( $\left.\mathrm{I}_{\text {trans }}\right)$, due the response of the circuitry on abrupt changes in current.

$$
\begin{gathered}
I_{\text {total }}=I_{\text {int }}+I_{\text {disp }}+I_{o f f}+I_{\text {trans }} \\
I_{\text {disp }}=C_{\text {par }} \frac{d V}{d t}
\end{gathered}
$$

Different strategies are proposed to reduce the effects of capacitance,[14,17] but a simple data processing procedure can be performed to correct for the displacement current. The procedure is based on the change of sign of the displacement current for forward and backward directions, respectively. Thus, simply adding forward and backward currents for each voltage in an appropriate voltage regime will cancel out $\mathrm{I}_{\text {disp }}$, and the resulting summed currents yields $2 \mathrm{I}_{\text {off }}$, so both the offset and displacement currents can be accounted for. As $\mathrm{I}_{\text {dis }}$ and $\mathrm{I}_{\text {off }}$ are not constant, which can be determined once and then can be compensated for the given measurement system but rather changes with time, location on the surface, and measurement range, this procedure have to be performed for every point of the grid for the FORC-IV measurements described in section 5.2. Such approach would additionally improve reproducibility and sensitivity of cAFM IV measurements.

\subsection{Experimental}

The cathode films were grown from a sintered $\mathrm{LiMn}_{2} \mathrm{O}_{4}(10 \mathrm{wt} \%$ excess $\mathrm{Li})$ target at an oxygen pressure of $0.133 \mathrm{mbar}$, substrate temperature of $600{ }^{\circ} \mathrm{C}$, deposition frequency of $2 \mathrm{~Hz}(\sim 0.08 \AA /$ pulse), on Platinum (Pt) sputtered over Si, and Nb:STO with the (100), (110), and (111) crystal orientations. The VAN film was grown from a sintered $67 \%$ $\mathrm{La}_{0.5} \mathrm{Li}_{0.5} \mathrm{TiO}_{3}+33 \% \mathrm{LiMn}_{2} \mathrm{O}_{4}(30 \mathrm{wt} \%$ excess $\mathrm{Li})$ target at an oxygen pressure of 0.2 mbar, substrate temperature of $850{ }^{\circ} \mathrm{C}$, deposition frequency of $20 \mathrm{~Hz}(\sim 0.15 \AA /$ pulse), on STO(100) layer, where the first $4 \mathrm{~nm}$ was deposited on Si by molecular beam epitaxy (MBE) and $40 \mathrm{~nm}$, by PLD. A KrF excimer laser was used operating at $248 \mathrm{~nm}$ and laser

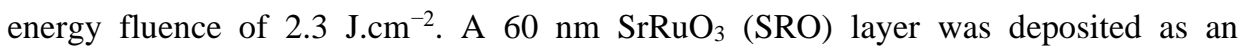
intermediate layer for both samples to enhance the electrical transport between the $\mathrm{LiMn}_{2} \mathrm{O}_{4}$ cathode and the conducting $\mathrm{Nb}: \mathrm{STO}$ substrate.[12,18] The Nb:STO substrates (0.5 wt \%,$a=3.905 \AA$ )[19] were annealed in a tube-oven before the deposition at 950 ${ }^{\circ} \mathrm{C}$ for $90 \mathrm{~min}$ in an oxygen flow of $150 \mathrm{~L} / \mathrm{h}$. After deposition, the films were cooled down to room temperature in an oxygen pressure of $0.2 \mathrm{mbar}$ at a rate of $10{ }^{\circ} \mathrm{C} / \mathrm{min}$. To examine the crystal structure of the films, X-ray diffraction (XRD) measurements were performed 
on a PANalytical X'Pert PRO diffractometer, with a PIXcel1D detector and a $\mathrm{Cu}$ source $(\lambda=1.5406 \AA)$.

(a)

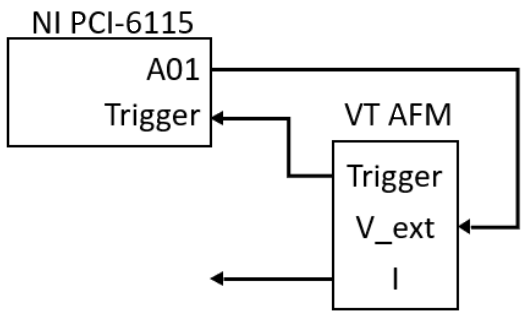

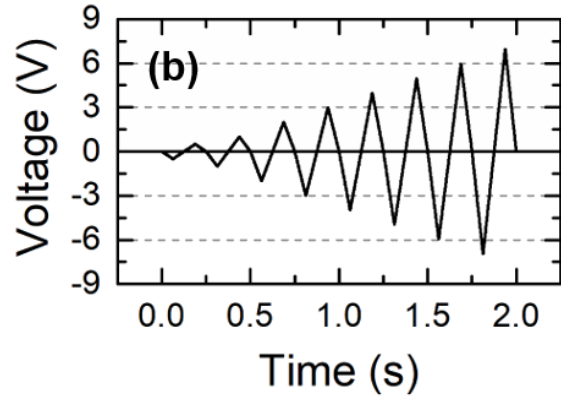

Figure 5-2. (a) Configuration used for the FORC-IV technique and (b) bias applied by NI PCI-6116 from the A01 port into the V_ext at the VT-AFM.

The current-voltage measurements have been performed on an Omicron VT-AFM atomic force microscope at pressures below $10^{-10}$ mbar interfaced with National Instrument card (NI PCI-6115) controlled by LabView. Nanosensors' PPP-EFM-SPL n ${ }^{+}$ silicon cantilevers with conductive $\mathrm{Pt} / \mathrm{Ir}$ coated tip were used $(\mathrm{k}=0.5-9.5 \mathrm{~N} / \mathrm{m})$. For the $\mathrm{Nb}$ :STO films, the substrate is used as bottom contact, while for films grown on buffered Silicon, Pt and SRO were used as contacts for each specific case. The setup and interface are displayed in Figure 5-2a, together with the voltage signal applied, in Figure 5-2b. The bipolar wavelength assists in restoring the initial state of the pixel and its surroundings, eliminating the memory of adjacent pixels.[5] The current data acquired was analyzed using a custom Matlab program, where the capacitance is compensated by the method described in section 5.2.1, the IV hysteresis loop area is calculated by the Matlab function polyarea and, to make the area of each loop comparable,[5] a normalization procedure was carried out where the normalized area is given by $\mathrm{N}=\mathrm{A} /\left(\mathrm{I}_{\max } \mathrm{V}\right)$, where $V$ is applied bias and $I_{\max }$ is the maximum current obtained for each loop.

\subsection{Local Electrochemistry in $\mathrm{LiMn}_{2} \mathrm{O}_{4}$ cathode thin films}

For implementing the technique, LMO was used as a model system. From chapter 2, LMO can be grown in highly crystalline films, and such films' orientations can be easily tuned by means of changing the crystal structure of the bottom electrode.[12] Local electrochemistry of polycrystalline films of LMO have been investigated by Kholkin et al. [20,21] via ESM measurements. Epitaxial thin films provide the capability to study specific orientation of the crystal facets on the surface, which polycrystalline films make inaccessible. As aforementioned, ESM is better applied in pure ionic conductors, thus FORC-IV will be applied here. 


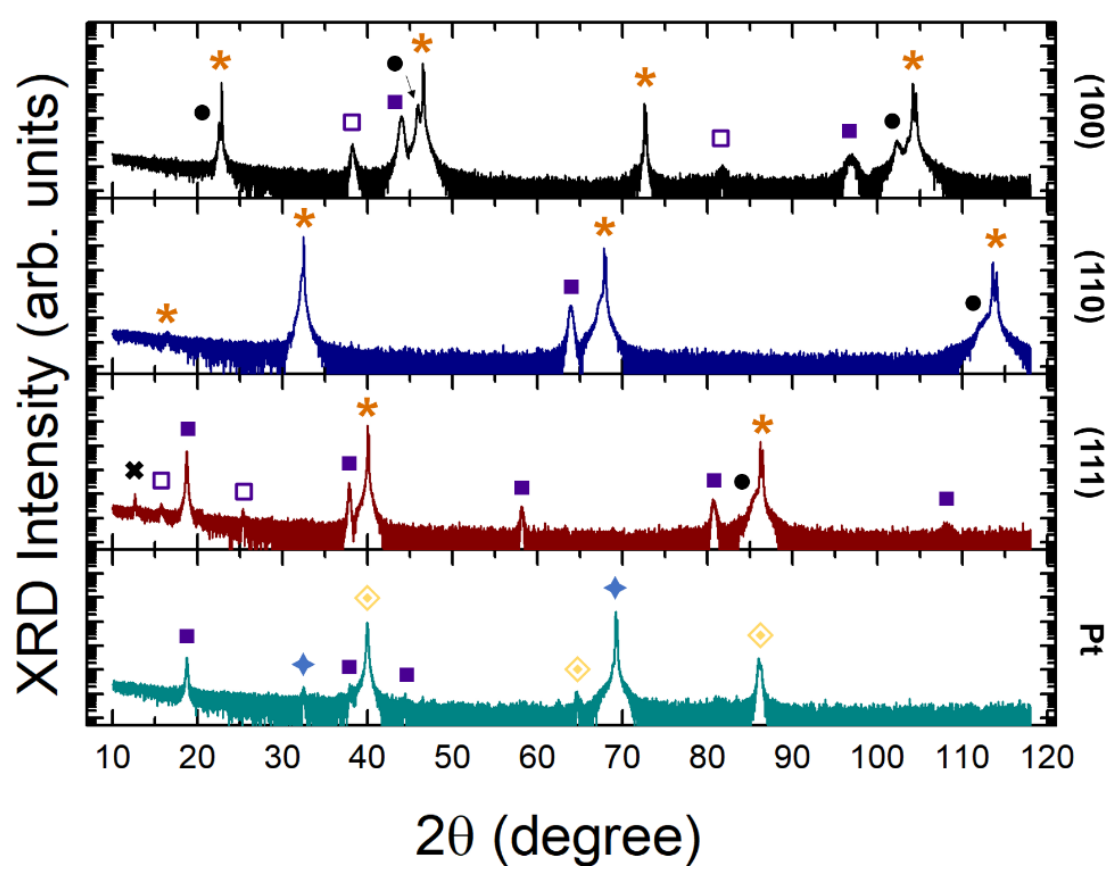

Figure 5-3. Full XRD pattern of the LMO films grown with the same conditions on STO substrates with different crystallographic orientations and on Pt. LMO peaks are shown by $\boldsymbol{\square}$, the STO substrate peaks by $\star$, SRO by $\bullet$, Si by $\downarrow$, and Pt by $\diamond$. Impurity phases are indicated by $\square$, corresponding to $\mathrm{Mn}_{2} \mathrm{O}_{3}$ and $\mathbf{x}$, to $\mathrm{Sr}_{2} \mathrm{RuO}_{4}$.

The structural quality of the LMO films was investigated by XRD analysis, as shown in Figure 5-3. The three types of LMO films grown on Nb:STO substrates with different orientations exhibit coherent growth in which the out-of-plane crystal orientation of the films is aligned with the orientation of the substrate. The $\mathrm{LMO}(\mathrm{Pt})$ displays a combination of both (111) and (100) orientation, which is expected considering that for this spinel, the $\langle 111\rangle$ crystal facets is the lowest energy state surface,[22] and the $\langle 100\rangle$ facet displays formation energy close to that of the $\langle 111\rangle .[23]$

As aforementioned, a $60 \mathrm{~nm} \mathrm{SrRuO}_{3}$ (SRO) layer was deposited as an intermediate layer. The $\mathrm{LMO}(110)$ and $\mathrm{LMO}(\mathrm{Pt})$ films show the presence of highly crystalline epitaxial layers, without any impurity phase, in good agreement with previous studies of LMO growth on STO substrates.[18,24] This suggests that the PLD process parameters (e.g. temperature, pressure, laser energy density, target composition) were optimized successfully to correct for any loss of volatile lithium during ablation, nucleation or growth. Interestingly, the $\mathrm{LMO}(100)$ and $\mathrm{LMO}(111)$ show minor contributions of a secondary phase, although all four LMO films were grown during the same deposition procedure. The extra peaks suggest the presence of a small amount of $\mathrm{Mn}_{2} \mathrm{O}_{3}$, [25] which 
could be due to the enhanced lithium volatility at the (100) surface of $\mathrm{LiMn}_{2} \mathrm{O}_{4}$ as mentioned in chapter 2.[26]

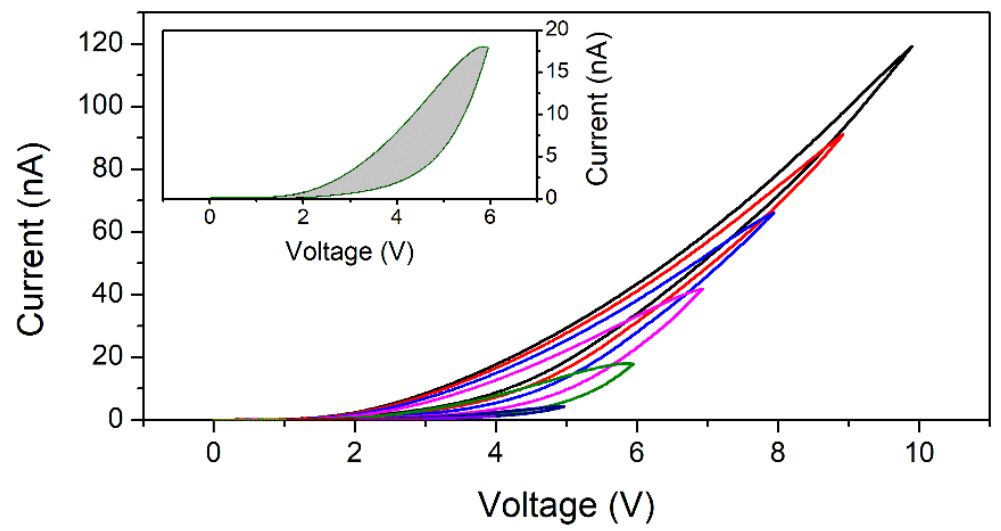

Figure 5-4. Current as a function of voltage for each $V_{p}$ from the FORC-IV measurement on the $\mathrm{LMO}(100)$ sample. Different $\mathrm{V}_{\mathrm{p}}$ are represented by different colors. The IV curves are an average of all measured pixels. (inset) Current as a function of voltage for $\mathrm{V}_{\mathrm{p}}=6$ $\mathrm{V}$. The area of the graph is highlighted.

The positive portion of the current as a function of the voltage applied (Figure 5-2b) obtained for the LMO(100) sample is displayed in Figure 5-4. Each $\mathrm{V}_{\mathrm{p}}$ is plotted with a different color for clarity, and these curves are an average of the whole scanned area. It is possible to notice that up to $\mathrm{V}_{\mathrm{p}}=6 \mathrm{~V}$ no hysteresis loop is observable. This reversible behavior indicates that only electronic conduction is responsible for the current. As the voltage increases, ions start to participate on the total conduction and the IV loop begins to display a hysteresis, depending on the scanning rate. This area, highlighted on the inset of Figure 5-4, is proportional to the amount of ions involved on the conduction and, in order to make it comparable for different $\mathrm{V}_{\mathrm{p}}$, it is normalized by the procedure introduced in the Experimental section. The normalized area, when plotted versus $V_{p}$, yields a curve in which the peak is used on the peak voltage maps.

The results obtained by the FORC-IV measurement, are displayed in Figure 5-5. The morphology images (Figure 5-5a-d), current data (Figure 5-5e-h) and peak voltage maps (Figure 5-5i-1) were obtained simultaneously. The measurement was performed in a $63 \times 63$ grid, resulting in a pixel size of $\sim 16 \mathrm{~nm}^{2}$. The current maps display the maximum value on the IV loop for the $7 \mathrm{~V}_{\mathrm{p}}$ applied by the waveform in Figure 5-2b. Although in this case spectroscopic maps look like the current maps (due to the inherent connection between the Li-ion dynamics and conductivity in LMO), they reveal different type of information. Specifically, electrochemical activity can be directly visualized in the loop area maps but cannot be directly extracted from the current map. 

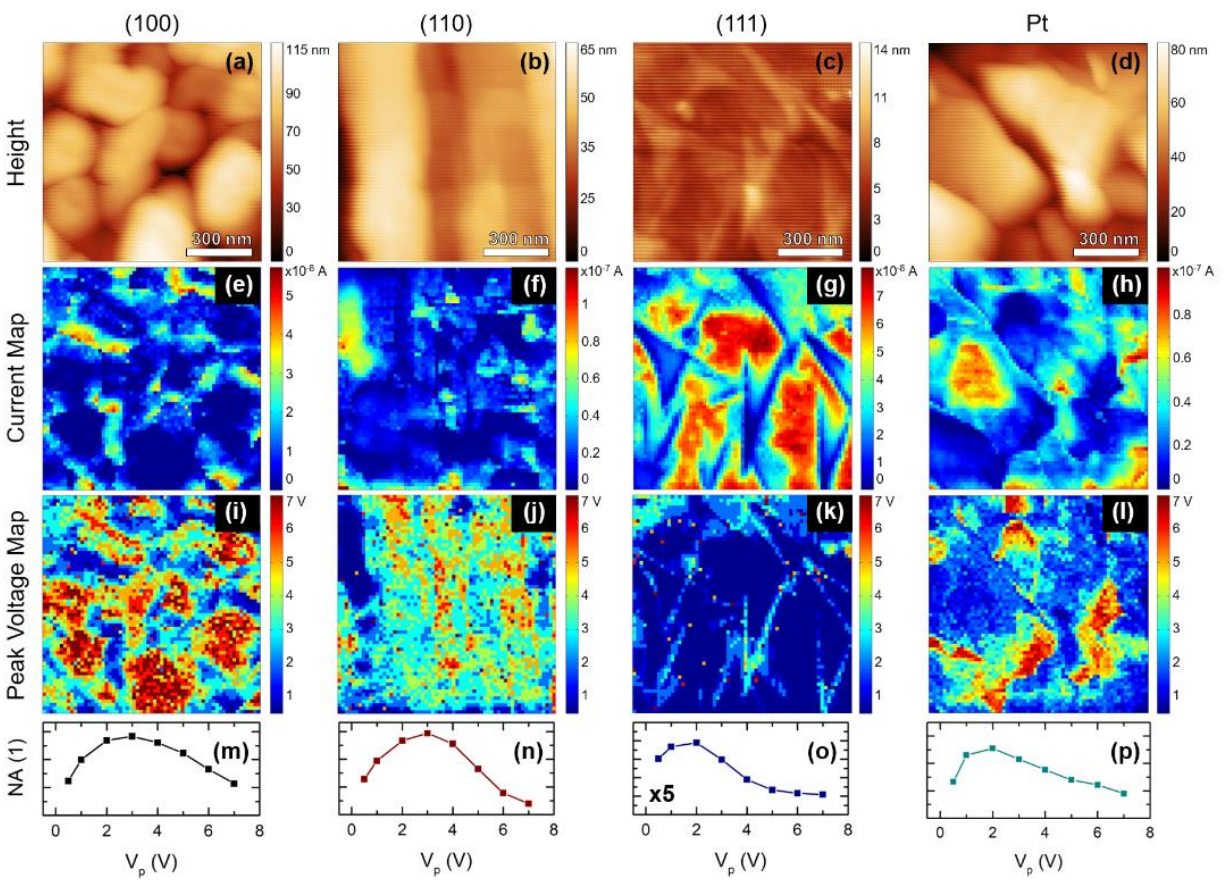

Figure 5-5. FORC-IV analysis displaying the (a-d) AFM images with the morphology of the area scanned (e-h) current maps with the maximum current measured at the $7 \mathrm{~V}$ loop, (i-l) peak voltage maps where the voltage for the maximum normalized area value is plotted for every pixel and, (m-o) average normalized area per peak voltage curves for STO substrates with different crystallographic orientations and Pt.

The normalized area versus peak voltage for each sample is plotted in Figure 5-5m-p. The curves in Figure 5-5m-p are an average for all the pixels measured, while in Figure 5-5i-1 the voltage where the maximum value occurs for the normalized area (NA) curve for each pixel is displayed. The contrast is given by the ease in each ions can move in certain areas, given that the peak in NA yields the voltage in which the ionic movement is more efficient. Thus, higher voltage (red colors) means more energy required for dislocating the ions. This fact can be explained by the predominantly electronic conductivity of the high-conductive state, as opposed to ion-controlled resistivity changes in the low-conductive state.[5]

For all the maps in Figure 5-5i-1, the $\langle 111\rangle$ facet is displayed in blue, which means higher ionic mobility. The $\langle 111\rangle$ facet could display a higher ionic mobility because, although the spinel structure presents 3D channels for Li-ion diffusion,[27] the conduction pathways are aligned along the (111) orientation resulting in direct channels for diffusivity at the $\langle 111\rangle$ facet, corroborating with the given contrast. These results could 
lead to the initial conclusion that the films grown along the (111) orientation would result in better electrochemical performance, which is not observed for these films (chapter 2). For batteries, surface area is an essential factor,[28] as discussed in chapters 2 and 4, due to the improved contact with the electrolyte resulting in more electrochemical reactions occurring concurrently. Thus, even if the films on the (111) orientation would display higher ionic mobility, their flat surface makes them unsuitable for the application as a cathode. The LMO thin film grown on Pt results in an averaged NA area with a peak at 2 $\mathrm{V}$ (as expected for the (111) orientation), but appears to display a minor peak a higher voltages $(\sim 6 \mathrm{~V})$ which could be a result of the combination of both (111) and (100) orientations present in the film, as shown by the XRD images in Figure 5-3.

Thus, the qualitative FORC-IV analysis, together with the previous knowledge in LMO cathode material, indicates that to improve the battery performance both surface area and the presence of the $\langle 111\rangle$ facets should be increased. To achieve that, the growth conditions of the $\operatorname{LMO}(100)$, which already displays the highest surface area, can be adjusted to favor smaller features, for example, through higher deposition frequencies.

\subsection{Electrochemistry of VAN at the nanoscale}

To study the electrochemistry of VANs at the nanoscale the films grown on Silicon buffered with crystalline STO(100) were chosen, which are the most promising for the applications in microbatteries.[29] The growth, composition, morphology and structure (XRD pattern displayed in Figure 5-6d, for ease) of this film are discussed in more details in chapter 4 . The results obtained by the FORC-IV measurement, are displayed in Figure 5-6. The morphology (Figure 5-6a), current data (Figure 5-6b), and peak voltage maps (Figure 5-6c) were obtained simultaneously. The measurement was performed in a $63 \times 63$ grid, resulting in a pixel size of $\sim 6.3 \mathrm{~nm}^{2}$. For this measurement, the waveform presented in Figure 5-2b was multiplied by a gain of 0.5 to avoid premature dielectric breakdowns or charge trapping,[17] resulting in the current map depicted in Figure 5-6a, where the maximum current value on the IV loop for the $3.5 \mathrm{Vp}$ is shown.

The peak voltage maps, depicted in Figure 5-6b show clear contrast with the majority of the map displaying the maximum of the NA at $3.5 \mathrm{~V}$. This result indicates that the maximum voltage used for the FORC-IV measurement was insufficiently low, and a higher voltage is required to obtain a full curve where the $V_{p}$ on which the maximum NA occurs can be extracted. For this measurement, higher voltages were not used as the sample displayed currents that exceeded the limits of the equipment. It is also worth noticing that not all pillars (displayed in Figure 5-6a) are conductive (Figure 5-6b) or show FORC-IV signal, which could be a result of the non-ideal contact between the cathode pillars and the current collector due to the presence of a thin LLTO layer in between, as discussed in chapter 4 . The same behavior is observed in other regions of the 
same film, as observed in Figures 5-5e and Figure 5-6f. Some regions of the LLTO matrix show low resistance and a FORC-IV signal, which is perhaps caused by the presence of multiple impurities phases, as shown by Figure 5-6d and discussed in more details in chapter 4 , although the conduction pathways that leads to this behavior would require further investigation.
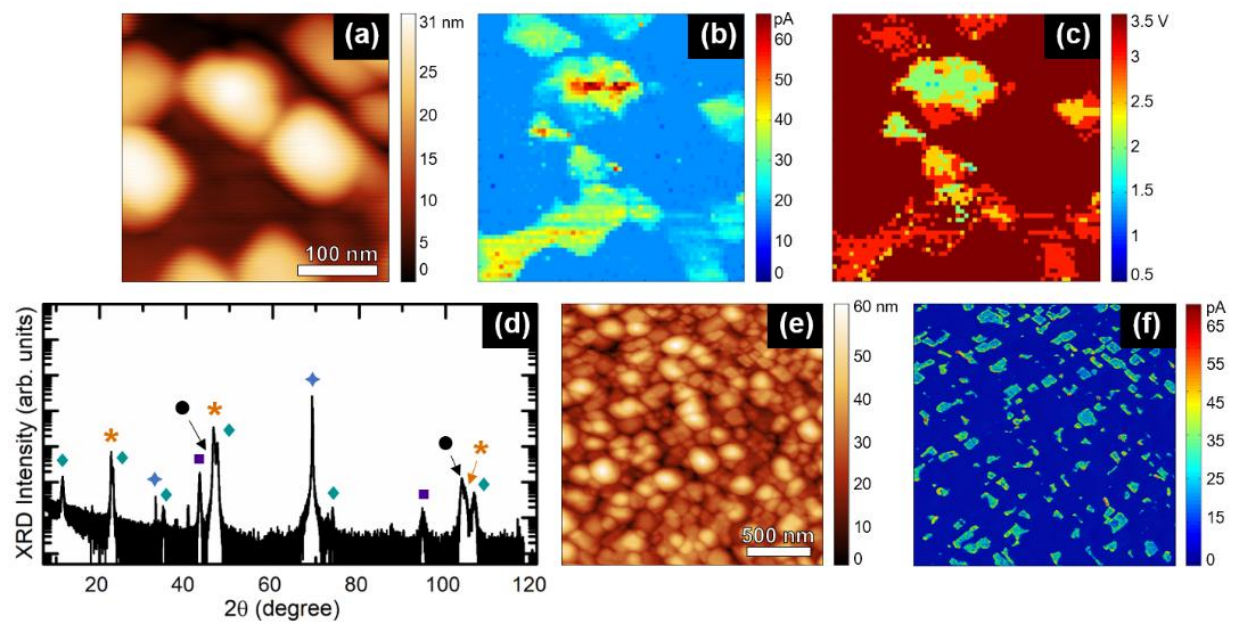

Figure 5-6. (a) AFM image with the morphology of the area scanned. (b) Current map with the maximum current measured at the $3.5 \mathrm{~V}$ loop. (c) peak voltage map where the voltage for the maximum normalized area value is plotted for every pixel. (d) Full XRD pattern of the VAN films grown on STO(100)-buffered Silicon substrate, in which LLTO

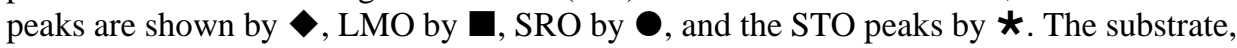
$\mathrm{Si}(001)$, is indicated by $\uparrow$. Impurities phases as $\mathrm{Mn}_{2} \mathrm{O}_{3}$ and rutile $\mathrm{TiO}_{2}$ are present but not indicated. (e) AFM image of a larger area. (f) Current map at the larger area at $4 \mathrm{~V}$.

The pillars that show FORC-IV signal, display two distinctive regions: the middle with higher ionic conductivity (green) and edges with slightly lower ionic conductivity (orange). Hirayama et al. show the formation of a possible interphase between thin films of LLTO and LMO (111)-oriented grown by PLD,[30] formed by the diffusion of oxygen and titanium between the layers, which could reduce the active material volume, affecting the electrochemical behavior of this film as shown in chapter 4. In a multilayer film, such reactions could be mitigated by buffer layers of a material that does not react with the materials of interest and that wouldn't hinder the efficient of the layers. In the case of VANs, such approach would require a third material to grow around the pillars, shielding them from the matrix, however the growth self-assembled three-component VANs have not been widely explored.[31] Another method is to investigate different orientations that could provide a more stable interface [24] or explore new combinations of cathode and electrolyte that wouldn't show such chemical reaction. 


\subsection{Prospects for the analysis of a complex ionic/electronic system}

The FORC-IV spectroscopy analysis is a powerful method to measure the electrochemistry at the nanoscale, however, the maps acquired only provide qualitative data over the local ionic conductivity. The versatility of the technique becomes evident as much more can be achieved beyond the qualitative analysis presented. In this section, techniques that can be used to give the next step on attaining physical meaning from the spatial IV curves obtained are introduced and recommended for future work.

\subsubsection{Clustering and Unmixing}

In order to decorrelate a high-dimensional FORC-IV data set in a way that allows physical interpretation, different techniques can be used and have been proposed.[32] To know what is the smallest reasonable number of behaviors present in the system, k-means clustering is a powerful technique used in datamining, and can be used for unmixing, considered all components are linear. As an advanced unmixing technique, Bayesian Linear Unmixing will be introduced.

\section{k-means}

The k-means clustering is a method of vector quantization, originally from signal processing, that is popular for cluster analysis in data mining, that will be used to know what is the minimum number of components in the system and unmixing them. The initial simple hypothesis is that the number of behaviors is equal to the number of materials in the nanocomposite. However, MIECs provide different types of conduction (electronic and ionic), and the interface might display a different kind of conductive behavior, as shown in section 5.5.

To help establish the relevant number of behaviors, k-means clustering can be employed in a full spectrographic data set.[33] The k-means algorithm separates $M$ points that exist in $N$ dimensions into a specified $k$ number of clusters of curves that have similar behavior so that the sum of squares within a cluster is minimized, described by Equation (5.3)[34,35] Thus, given a set of observations $\left(\mathrm{x}_{1}, \mathrm{x}_{2}, \ldots, \mathrm{x}_{\mathrm{M}}\right)$, where each observation is a $\mathrm{N}$-dimensional real vector, $\mathrm{k}$-means clustering aims to partition the $M$ observations into $k(\leq \mathrm{M})$ sets $\mathrm{S}=\left\{\mathrm{S}_{1}, \mathrm{~S}_{2}, \ldots, \mathrm{S}_{\mathrm{k}}\right\}$ so as to minimize the within-cluster sum of squares (i.e. variance). 


$$
\arg \min \sum_{i=1}^{k} \sum_{x_{j} \in S_{i}}\left\|x_{j}-\mu_{i}\right\|^{2}
$$

where $\mu_{i}$ is the mean of points in $S_{i}$. For the FORC-IV measurements proposed, $M$ is the set of data acquired for each individual point with the IV dimensions (N), and $S$ is set of components that the IV curve can be decomposed to. As a minimization parameter, square Euclidian distance is used, with each centroid being the component-wise median of the points in a given cluster. The k-means algorithm divides the data set in a specified number of optimally selected clusters, with the number of clusters as an input.

To evaluate the quality of the separation as a function of the number of clusters, the data can be presented in the form of a dendrogram, where all observations are grouped into a single cluster initially and are recursively separated down the hierarchy. This is achieved by establishing a distance metric between observations and linkage criteria used to find the dissimilarity of clusters as a function of pairwise distances. A larger vertical drop in each of the binary branches of the dendrogram tree, indicates a better cluster classification scheme in the data. Therefore, the number of clusters can be obtained based on the linkage analysis and used as an input to the k-means clustering method.
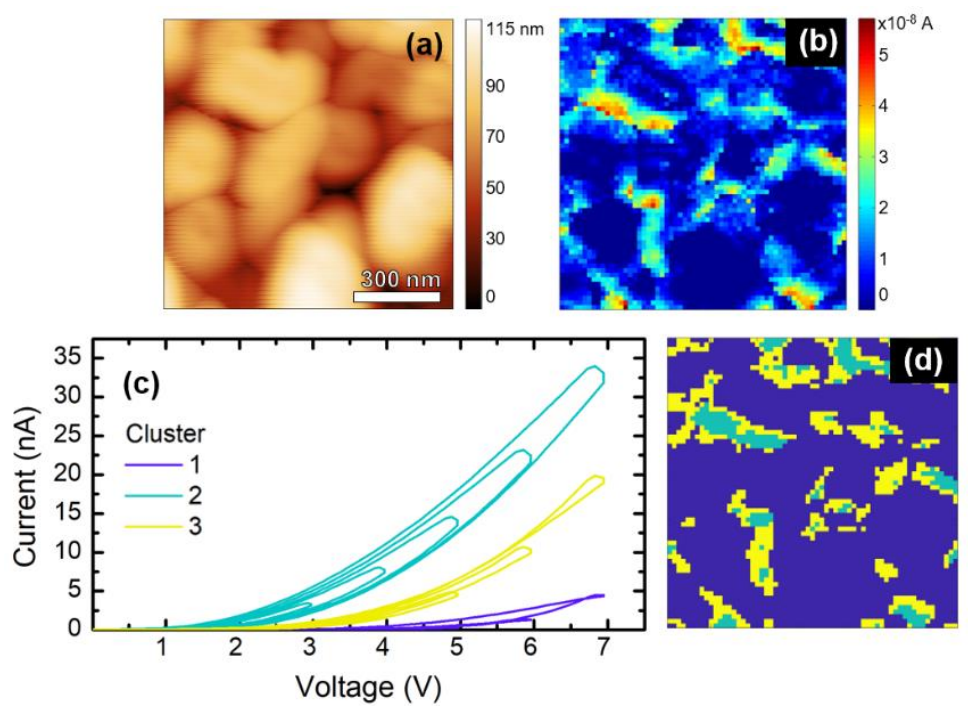

Figure 5-7. (a) AFM image with the morphology of the area scanned, (b) current map with the maximum current measured at the $7 \mathrm{~V}$ loop, (c) IV curves for three different clusters and, (d) cluster distribution over the scanned area. 
An example of how k-means clustering can be used is shown in Figure 5-7. The data used is the same as presented in Figure 5-5 for the FORC-IV analysis on LMO(100). Figure 5-7a-b display the morphology of the area scanned and the current map at $7 \mathrm{~V}$, respectively. Here, three clusters were used for partitioning the IV loops, and three distinct profiles were found, as shown in Figure 5-7c. The IV loops presented in Figure 5-7c are an average of all the IV loops that compose each cluster. Their spatial distribution over the scanned area is depicted in Figure 5-7d. The regions can be divided into low conductivity (cluster 1, blue), intermediate conductivity (cluster 2, yellow) found on the interface between clusters 1 and 3, and high conductivity (cluster 3, cyan) where hysteretic behavior is more pronounced.

\section{Bayesian Linear Unmixing (BLU)}

The FORC-IV spectroscopy method yields a 4D dataset, where $\mathrm{I}=f\left(\mathrm{x}, \mathrm{y}, \mathrm{V}, \mathrm{V}_{\mathrm{p}}\right)$, that contains information on the nanoscale transport and electrochemistry of the sample. Although k-means is a powerful tool to analyze the distribution of different components over the area of interest, to extract statistical behavior that can be understood physically, i.e. separate the data into well-defined clusters with clear spectroscopy behavior that has an intensity weight component providing insight into the spatial distribution of the behavior. This analysis can be achieved by Bayesian linear unmixing.[36]

Bayesian linear unmixing (BLU) [36-38] describes the dataset (Y) as a linear combination of position-independent endmembers (M) with respective relative abundances (A) corrupted by the additive Gaussian noise (N), as described by Equation (5.4):

$$
Y=M A+N
$$

This method features several built-in constraints that allow for the physical interpretation of results, namely the non-negativity $\left(\mathrm{M}_{\mathrm{i}} \geq 0, \mathrm{~A}_{\mathrm{i}} \geq 0\right)$, full additivity, and sum-to-one $\left(\sum \mathrm{A}_{\mathrm{i}}=1\right)$ constraints for both the endmembers and the abundance coefficients.[36]

The algorithm estimates the initial projection of end-members in a dimensionalityreduced subspace (PCA) via N-FINDR,[39] which finds a simplex of maximum volume that can be inscribed within the hyperspectral data set using a simple nonlinear inversion. The end-member abundance as well as noise variance are chosen by a multivariate Gaussian distribution, where the posterior distribution is calculated based on end-member independence using Markov chain Monte Carlo, which generates asymptotically distributed samples probed by Gibbs sampling strategy. The resulting end-members $M$ 
are non-negative, and respective abundances add up to 1 . Thus, the spectrum at each location is decomposed into a linear combination of spectra of individual components in corresponding proportions. These particular constraints make transition from statistical analysis to physical behavior significantly more straightforward, as the currents in the samples of interest are additive.[32]

By making the abundances additive and the end-members positive, physical behavior to the shape and nature of the end-member curves can be assigned. The analysis of these curve's loading map adds the spatial component to the behavior that nonstatistical methods of analysis generally do not allow. An additional unique aspect of Bayesian analysis is that the endmember spectra and abundances are estimated jointly in a single step, unlike multiple least-squares regression methods, where initial spectra should be known.[36]

\subsubsection{Temperature Dependence}

To extract physical meaning from the FORC-IV measurements, it is crucial to know the type of conduction mechanisms present in the films analyzed and, since most of such mechanisms are thermally activated, temperature dependence measurements are required,[5] yielding a $5 \mathrm{D}$ dataset, i.e. $\mathrm{I}=f\left(\mathrm{x}, \mathrm{y}, \mathrm{V}, \mathrm{V}_{\mathrm{p}}, \mathrm{T}\right)$. Once the current measured is decoupled, given the methods presented in section 5.6.1, the different endmembers components can be fitted to conduction mechanism models and the physical properties, e.g. potential barrier for ionic conduction in the films, can be obtained.

The parameter used for discussion throughout this thesis, as the property that requires improvement in the cathode thin films, ionic conductivity will be used as an example of thermally activated conductivity. Ionic conduction results from the movement of ions under an applied electric field. The movement of the ions may come from the existence of lattice defects in the films, or due to the influence of external electric field resulting in the jump of ions over a potential barrier from one site to another.[40] If the area of the AFM tip used for the IV measurements is known, the current density $(\mathrm{J})$ can be calculated, i.e. the measured current normalized by the electrode area, and the ionic conduction current can be expressed by Equation 5.5.[40]

$$
J=J_{0} \exp \left[\frac{-1}{k_{B} T}\left(q \varphi_{B}-0.5 E q d\right)\right]
$$

where $J_{0}$ is the proportional constant, $q \varphi_{B}$ is the potential barrier height, $E$ is the applied electric field, i.e. the applied voltage normalized by the thickness of the film, $d$ is the 
spacing of two nearby jumping sites, $k_{B}$ is the Boltzmann's constant, $q$ is the electronic charge and $T$ is the absolute temperature.

Temperature dependence measurements will naturally create difficulties in the FORCIV spectroscopy, as thermal drift of the AFM scanner and sample expansion occur,[41] and will be most noticeable at the long scanning times and small scales required for the FORC-IV measurements. Thus, it is expected a reduction in resolution as the pixel area needs to be increased to compensate for the thermal drift.

The first steps towards this direction were given and the results obtained are illustrated in Figure 7-8. Figure 7-8a shows the morphology of a scanned region of the LMO thin film grown on $\mathrm{Nb}: \mathrm{STO}(100)$. The current map obtained in the $9 \mathrm{~V}$ loop of a triangular waveform is displayed in Figure 7-8b. Due to thermal drift, as aforementioned, the resolution of the measurement is limited. In this case, the resolution of the scan is $10 \times 10$ pixels. The average current density versus electric field (JE) curves can be seen in Figure 5-8c. As expected from Equation 5.5, higher temperatures yields higher current densities; the larger area for the highest temperature also indicates larger ionic contribution to the measured current.
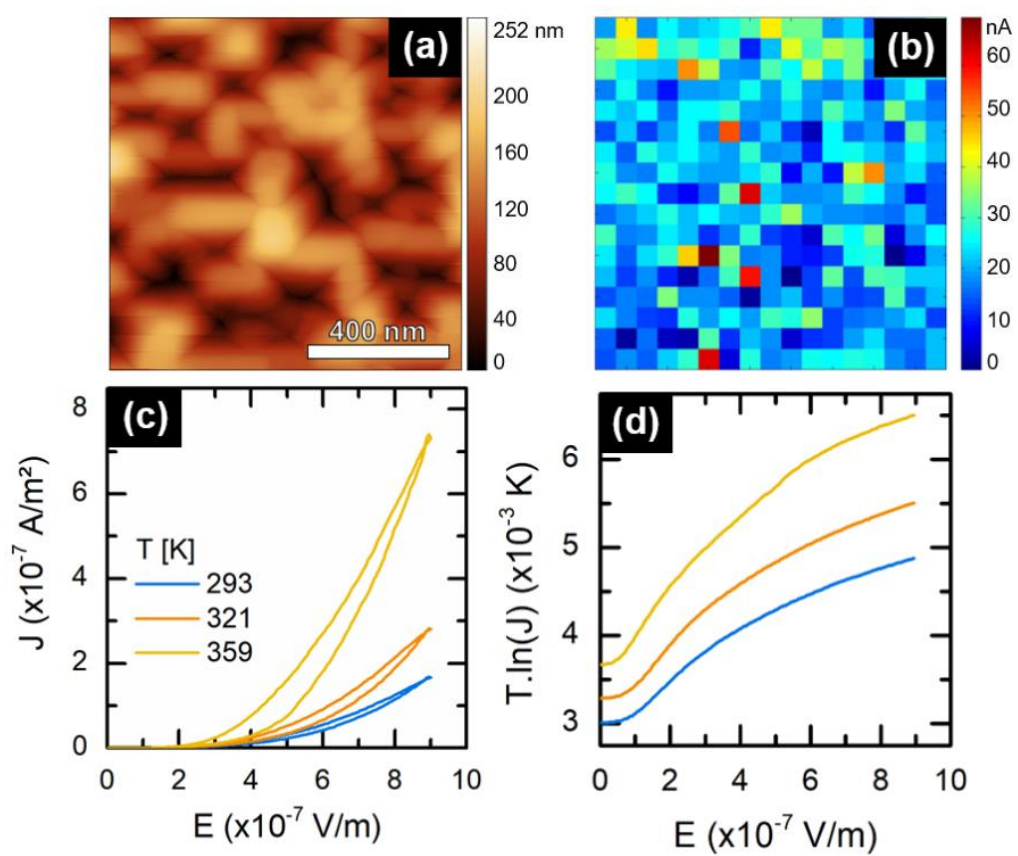

Figure 5-8. (a) AFM image with the morphology of the area scanned, (b) current map with the maximum current measured at room temperature for a $9 \mathrm{~V}$ loop, (c) average current density versus electric field curves for three different temperatures and, (d) plot of the $T \ln (J)$ as a function of the electric field. 
Figure 7-8d shows the onward portion of the JE curves plotted to fit the ionic motion expression, in Equation 5.5. Linearizing Equation 5.5, yields:

$$
T \cdot \ln \left(\frac{J}{J_{0}}\right)=-\frac{q \varphi_{B}}{k_{B}}+\frac{0.5 q d}{k_{B}} E
$$

Thus, applying a linear fit to the linear portions of the curves presented in Figure 5-8d, yields $d$ from the slope and $q \varphi_{B}$ from the constant portion.

\subsection{Conclusion}

The ability to image function on the nanoscale can be used to elucidate the relationship between crystal structure and functionality in nanostructured thin films with exceptional resolution. On this chapter FORC-IV was introduced, and the capability to measure electrochemistry at the nanoscale was demonstrated. Although the technique provides a merely qualitative analysis, this simple approach can be used to investigate battery performance on a scale not accessible by other techniques. For implementing the technique, LMO was used as a model system as it has been shown previously to be grown in crystalline films, and such films' orientations could be easily tuned by changing the crystal structure of the bottom electrode.[12] Although local electrochemistry of polycrystalline films of LMO have been investigated,[20,21] epitaxial thin films provide the capability to study specific orientation of the crystal facets on the surface, which polycrystalline films make inaccessible. The FORC-IV peak voltage maps show that for all films $\langle 111\rangle$ facet is displayed the highest ionic mobility, which could be result of ionic conduction pathways aligned along the (111) orientation. Although LMO(111) would provide the highest ionic mobility, this does not transfer directly to performance, as for batteries surface area is an essential factor.[28] Thus, through FORC-IV analysis, both surface area and the presence of the $\langle 111\rangle$ facets should be increased, which could be achieved by adjusting the growth paraments of the LMO(100), e.g. through higher deposition frequencies. The FORC-IV analysis on the LMO-LLTO VANs shows that the LMO pillars display two distinctive regions: the middle with higher ionic conductivity and edges with slightly lower ionic conductivity, which could be a result of an interphase formed between LMO and the LLTO.[30] Such phenomenon could be mitigated by a third material, shielding the pillars, or by exploring new orientations or material combinations that present more stable interfaces. Lastly, techniques to decorrelate the high-dimensional FORC-IV data set that would allow for a quantitative analysis have been proposed. Examples on how k-means clustering and temperature dependence measurements can be used were given, although more efforts are still required for the proper application of these methods. 


\section{Bibliography}

[1] Strelcov, E., Yang, S. M., Jesse, S., Balke, N., Vasudevan, R. K., Kalinin, S. V., Solidstate electrochemistry on the nanometer and atomic scales: The scanning probe microscopy approach. Nanoscale 2016, 8, 13838-13858.

[2] de Beeck, J. O., Labyedh, N., Sepúlveda, A., Spampinato, V., Franquet, A., Conard, T., Vereecken, P. M., Vandervorst, W., Celano, U., Nanoscale electrochemical response of lithium-ion cathodes: A combined study using C-AFM and SIMS. Beilstein J.

Nanotechnol. 2018, 9, 1623-1628.

[3] Balke, N., Jesse, S., Morozovska, A. N., Eliseev, E., Chung, D. W., Kim, Y., Adamczyk, L., García, R. E., Dudney, N., Kalinin, S. V., Nanoscale mapping of ion diffusion in a lithium-ion battery cathode. Nat. Nanotechnol. 2010, 5, 749-754.

[4] Kalinin, S., Balke, N., Jesse, S., Tselev, A., Kumar, A., Arruda, T. M., Guo, S., Proksch, R., Li-ion dynamics and reactivity on the nanoscale. Mater. Today 2011, 14, 548-558.

[5] Strelcov, E., Kim, Y., Jesse, S., Cao, Y., Ivanov, I. N., Kravchenko, I. I., Wang, C.-H. H., Teng, Y.-C. C., Chen, L.-Q. Q., Chu, Y. H., Kalinin, S. V., Probing Local Ionic Dynamics in Functional Oxides at the Nanoscale. Nano Lett. 2013, 13, 3455-3462.

[6] Strelcov, E., Belianinov, A., Hsieh, Y. H., Chu, Y. H., Kalinin, S. V., Constraining Data Mining with Physical Models: Voltage- and Oxygen Pressure-Dependent Transport in Multiferroic Nanostructures. Nano Lett. 2015, 15, 6650-6657.

[7] Hsieh, Y.-H. H., Strelcov, E., Liou, J.-M. M., Shen, C.-Y. Y., Chen, Y.-C. C., Kalinin, S. V., Chu, Y.-H. H., Electrical Modulation of the Local Conduction at Oxide Tubular Interfaces. ACS Nano 2013, 7, 8627-8633.

[8] Kim, Y., Strelcov, E., Hwang, I. R., Choi, T., Park, B. H., Jesse, S., Kalinin, S. V., Correlative multimodal probing of ionically-mediated electromechanical phenomena in simple oxides. Sci. Rep. 2013, 3, 1-7.

[9] Balke, N., Maksymovych, P., Jesse, S., Herklotz, A., Tselev, A., Eom, C.-B., Kravchenko, I. I., Yu, P., Kalinin, S. V, Differentiating Ferroelectric and Nonferroelectric Electromechanical Effects with Scanning Probe Microscopy. ACS Nano 2015, 9, 6484-6492.

[10] Balke, N., Jesse, S., Li, Q., Maksymovych, P., Baris Okatan, M., Strelcov, E., Tselev, A., Kalinin, S. V, Current and surface charge modified hysteresis loops in ferroelectric thin films. J. Appl. Phys. 2015, 118, 72013.

[11] M.Thackeray, M., Thackeray, M. M., Manganese oxides for lithium batteries. Prog. Solid State Chem. 1997, 25, 1-71.

[12] Hendriks, R., Cunha, D. M., Singh, D. P., Huijben, M., Enhanced Lithium Transport by Control of Crystal Orientation in Spinel $\mathrm{LiMn}_{2} \mathrm{O}_{4}$ Thin Film Cathodes. ACS Appl. Energy Mater. 2018, 1, 7046-7051.

[13] Verde, M. G., Baggetto, L., Balke, N., Veith, G. M., Seo, J. K., Wang, Z., Meng, Y. S., Elucidating the Phase Transformation of $\mathrm{Li}_{4} \mathrm{Ti}_{5} \mathrm{O}_{12}$ Lithiation at the Nanoscale. ACS 
Nano 2016, 10, 4312-4321.

[14] Olbrich, A., Ebersberger, B., Boit, C., 1998 IEEE International Reliability Physics Symposium Proceedings. 36th Annual (Cat. No.98CH36173). 1998, pp. 163-168.

[15] Lányi, Š., Effect of tip shape on capacitance determination accuracy in scanning capacitance microscopy. Ultramicroscopy 2005, 103, 221-228.

[16] Tran, T., Oliver, D. R., Thomson, D. J., Bridges, G. E., “Zeptofarad” (10-21 F) resolution capacitance sensor for scanning capacitance microscopy. Rev. Sci. Instrum. $2001,72,2618-2623$.

[17] Rommel, M., Jambreck, J. D., Lemberger, M., Bauer, A. J., Frey, L., Murakami, K., Richter, C., Weinzierl, P., Influence of parasitic capacitances on conductive AFM I-V measurements and approaches for its reduction. J. Vac. Sci. Technol. B, Nanotechnol. Microelectron. Mater. Process. Meas. Phenom. 2013, 31, 01 A108.

[18] Suzuki, K., Kim, K., Taminato, S., Hirayama, M., Kanno, R., Fabrication and electrochemical properties of $\mathrm{LiMn}_{2} \mathrm{O}_{4} / \mathrm{SrRuO}_{3}$ multi-layer epitaxial thin film electrodes. J. Power Sources 2013, 226, 340-345.

[19] Herger, R., Willmott, P. R., Bunk, O., Schlepütz, C. M., Patterson, B. D., Delley, B., Shneerson, V. L., Lyman, P. F., Saldin, D. K., Surface structure of $\mathrm{SrTiO}_{3}(001)$ ). Phys. Rev. B - Condens. Matter Mater. Phys. 2007, 76, 195435.

[20] Luchkin, S. Y., Romanyuk, K., Ivanov, M., Kholkin, A. L., Li transport in fresh and aged $\mathrm{LiMn}_{2} \mathrm{O}_{4}$ cathodes via electrochemical strain microscopy. J. Appl. Phys. 2015, 118.

[21] Alikin, D. O., Ievlev, A. V., Luchkin, S. Y., Turygin, A. P., Shur, V. Y., Kalinin, S. V., Kholkin, A. L., Characterization of $\mathrm{LiMn}_{2} \mathrm{O}_{4}$ cathodes by electrochemical strain microscopy. Appl. Phys. Lett. 2016, 108.

[22] Kim, J. S., Kim, K., Cho, W., Shin, W. H., Kanno, R., Choi, J. W., A truncated manganese spinel cathode for excellent power and lifetime in lithium-ion batteries. Nano Lett. 2012, 12, 6358-6365.

[23] Kim, S., Aykol, M., Wolverton, C., Surface phase diagram and stability of (001) and (111) $\mathrm{LiMn}_{2} \mathrm{O}_{4}$ spinel oxides. Phys. Rev. B - Condens. Matter Mater. Phys. 2015, 92, 7 10.

[24] Hirayama, M., Sonoyama, N., Ito, M., Minoura, M., Mori, D., Yamada, A., Tamura, K., Mizuki, J., Kanno, R., Characterization of Electrode/Electrolyte Interface with X-Ray Reflectometry and Epitaxial-Film $\mathrm{LiMn}_{2} \mathrm{O}_{4}$ Electrode. J. Electrochem. Soc. 2007, 154, A1065.

[25] Xia, H., Wan, Y., Yan, F., Lu, L., Manganese oxide thin films prepared by pulsed laser deposition for thin film microbatteries. Mater. Chem. Phys. 2014, 143, 720-727.

[26] Amos, C. D., Roldan, M. A., Varela, M., Goodenough, J. B., Ferreira, P. J., Revealing the Reconstructed Surface of $\mathrm{Li}\left[\mathrm{Mn}_{2}\right] \mathrm{O}_{4}$. Nano Lett. 2016, 16, 2899-2906.

[27] Nitta, N., Wu, F., Lee, J. T., Yushin, G., Li-ion battery materials: Present and future. Mater. Today 2015, 18, 252-264.

[28] Cunha, D. M., Hendriks, T. A., Vasileiadis, A., Vos, C. M., Verhallen, T., Singh, D. P., Wagemaker, M., Huijben, M., Doubling Reversible Capacities in Epitaxial $\mathrm{Li}_{4} \mathrm{Ti}_{5} \mathrm{O}_{12}$ 
Thin Film Anodes for Microbatteries. ACS Appl. Energy Mater. 2019, 2, 3410-3418.

[29] Narayanan, V., Frank, M. M., Demkov, A. A., Thin Films on Silicon: Electronic and Photonic Applications. World Scientific Publishing 2016.

[30] Kim, S., Hirayama, M., Suzuki, K., Kanno, R., Hetero-epitaxial growth of $\mathrm{Li}_{0.17} \mathrm{La}_{0.61} \mathrm{TiO}_{3}$ solid electrolyte on $\mathrm{LiMn}_{2} \mathrm{O}_{4}$ electrode for all solid-state batteries. Solid State Ionics 2014, 262, 578-581.

[31] Kim, D. H., Sun, X. Y., Aimon, N. M., Kim, J. J., Campion, M. J., Tuller, H. L., Kornblum, L., Walker, F. J., Ahn, C. H., Ross, C. A., A three component self-assembled epitaxial nanocomposite thin film. Adv. Funct. Mater. 2015, 25, 3091-3100.

[32] Strelcov, E., Belianinov, A., Hsieh, Y. H., Jesse, S., Baddorf, A. P., Chu, Y. H., Kalinin, S. V., Deep data analysis of conductive phenomena on complex oxide interfaces: Physics from data mining. ACS Nano 2014, 8, 6449-6457.

[33] Haykin, S., Neural Networks: A Comprehensive Foundation. Prentice Hall PTR, USA 1994.

[34] MacQueen, J. B., Some Methods for Classification and Analysis of MultiVariate Observations. Proceedings of the Fifth Berkeley Symposium on Mathematical Statistics and Probability. University of California Press 1967.

[35] Hartigan, J. A., Wong, M. A., Algorithm AS 136: A K-Means Clustering Algorithm. J. R. Stat. Soc. Ser. C (Applied Stat. 1979, 28, 100-108.

[36] Dobigeon, N., Moussaoui, S., Coulon, M., Tourneret, J. Y., Hero, A. O., Joint Bayesian Endmember Extraction and Linear Unmixing for Hyperspectral Imagery. IEEE Trans. Signal Process. 2009, 57, 4355-4368.

[37] Dobigeon, N., Brun, N., Spectral mixture analysis of EELS spectrum-images. Ultramicroscopy 2012, 120, 25-34.

[38] Belianinov, A., Vasudevan, R., Strelcov, E., Ievlev, A., Steed, C., Yang, S. M., Tselev, A., Jesse, S., Biegalski, M., Shipman, G., Symons, C., Borisevich, A., Archibald, R., Kalinin, S., Erratum: Big data and deep data in scanning and electron microscopies: deriving functionality from multidimensional data sets. Adv. Struct. Chem. Imaging $2015,1,11$.

[39] Winter, M. E., Proc.SPIE. 1999.

[40] Chiu, F.-C., A Review on Conduction Mechanisms in Dielectric Films. Adv. Mater. Sci. Eng. 2014, 2014, 578168.

[41] Eaton, P., West, P., Atomic Force Microscopy. Oxford University Press, New York 2010. 


\section{Summary}

Tridimensional geometries for electrodes in solid-state batteries exhibit an improved energy vs. power balance, when compared to two dimensional electrodes. Additionally, the internal surface area between cathode, electrolyte and anode is enlarged, improving their current output. However, fabrication of such 3D batteries relies presently on the use of costly or multi-step methods. Vertically aligned nanocomposites (VANs) offer promising advantages over conventional planar multilayers as key functionalities are tailored by the strong coupling between the two phases and their interfaces. Epitaxial VANs are self-assembled through physical vapor deposition, without control of the deposition sequence, as is required for planar multilayer films. In this thesis, Licontaining VANs were self-assembled through pulsed laser deposition (PLD), for the spinel-perovskite system, for a solid-state battery application.

To elucidate the electrochemical behavior of specific crystal facets of battery materials, the control over the crystallographic properties of thin films via PLD was investigated. Improved control over the electrochemical properties of $\mathrm{Li}_{4} \mathrm{Ti}_{5} \mathrm{O}_{12}$ (LTO), $\mathrm{LiMn}_{2} \mathrm{O}_{4}$ (LMO) and $\mathrm{Li}_{0.5} \mathrm{La}_{0.5} \mathrm{TiO}_{3}$ (LLTO) thin films is enabled by structural engineering, which is unique for epitaxial thin films and cannot be obtained in single-crystal or polycrystalline samples. Dramatic differences in surface morphology were achieved by controlling the specific crystal orientation for the spinel LTO and LMO thin films. The exposed surface is predominantly $\langle 111\rangle$ for all obtained morphologies and showed to enhance the lithium storage of spinel thin film electrodes due to significant surface contributions. All three crystal orientations showed high electrochemical performance with good cyclability without any significant capacity fading, and high discharge capacities. The layer thickness dependence study demonstrated that the large capacities are caused by large surface contributions in lithium storage, where the (100)-oriented films exhibited the highest surface area. Structural engineering of the LLTO thin films showed the orientation dependence on the ionic diffusion for the solid electrolyte, as the Li-diffusion pathways are oriented in-plane, considering the tetragonal unit cell. Activation energies for hopping were calculated by a temperature dependence measurement, highlighting the contribution of La-rich layers on the diffusivity through the domain boundaries of thin films. Finally, with the knowledge acquired for the three components of the battery, a full solid-state battery stack was grown. X-ray diffraction measurements show the correct phases of LMO and LLTO. LTO showed a Li-deficient phase, which could be the result of the higher temperatures required for the LLTO growth. Electrochemical measurements do not reveal a short circuit between the layers, although cycling the full stack was not possible. Optimization of these batteries highlights the necessity in developing an intimate combination with a solid electrolyte in a vertically aligned 3D nanocomposite. 
Since no lithium-based VANs have yet been explored for energy storage, a model was developed to predict the formation of these structures. Furthermore, the simulations were used to analyze the influence of temperature and deposition rate on the morphology evolution of these nanocomposites. Successful use of kinetic Monte Carlo simulations (KMCS) to model the growth of lithium-based vertically aligned nanocomposite films was demonstrated, consisting of a promising LMO-LLTO cathode-electrolyte combination towards 3D solid-state batteries. A more realistic and low computational cost model was applied, where a minimal number of restrictions was used, and for which the interaction energies were based on experimental reflection high-energy electron diffraction (RHEED) measurements. The simulations showed good agreement with the films obtained experimentally and followed the trends for deposition rate and temperature dependencies predicted by physical models. Quantitative analysis exhibited good agreement with respect to the evolution of nanopillar density upon changes in synthesis parameters. The difference observed is explained by the choice of energies used in the KMCS modeling to minimize the computational time. The results demonstrate that KMCS modeling can be successfully applied to predict the nanopillar-matrix formation in lithium-based VAN films. However, the specific crystal structure and stoichiometry must be taken into consideration for the individual cathode and electrolyte materials, as well as for the interface formed between them.

The self-assembly procedure was applied for the first time with lithium containing materials to create electrode/electrolyte nanocomposites, deposited on crystalline substrates by PLD. The control over the crystallographic properties of VAN thin films and how different growth conditions influence the crystal structure, morphology, and composition of the nanopillars and matrix was demonstrated. The trend observed in the temperature dependence study suggested the formation of the Ti doped $\mathrm{LiMn}_{2-\mathrm{y}} \mathrm{Ti}_{\mathrm{y}} \mathrm{O}_{4}$ phase, with $y=1.5$, later confirmed by RSM maps and EELS analysis. The presence of $\mathrm{Ti}$ in the LMO pillars could be a result of the lower formation enthalpy of LMTO. The deposition rate, which can be used to tailor the pillar size of the VANs, was shown to barely affects the composition of the LMO phase. Different substrate orientations can be used to tailor the structure of the matrix and pillar phases and, consequently the morphology and interface between them. Based on experimental results and the simulations, the activation energy for hopping of the adatoms on the substrate surface can be tailored by means of different buffer layers, resulting in A-site and B-site termination. This switching in diffusivity was used to tune the pillar size and increase the pillar-current collector contact. The growth on Si substrate, buffered by a crystalline layer of STO, was shown, indicating the possibility of integration with $\mathrm{Si}$ processing. The expected characteristic peaks were not visible in the electrochemical analysis, suggesting that the VANs studied could provide a complex system beyond the capability of current electrochemical techniques. 
Advanced scanning probe microscopy (SPM) techniques allow measurements of electrochemistry on the nanoscale, which can be used to elucidate structure/function relationships in battery materials with exceptional resolution. To achieve insight into the non-uniform distribution of lithium activity, first order reversal curve current-voltage (FORC-IV) analysis was applied. The conduction mechanisms that rule the electrochemistry in the nanoscale were studied through local ionic diffusivity in LMO and VANs thin films epitaxially grown by PLD. The FORC-IV peak voltage maps showed that the $\langle 111\rangle$ facet shows highest ionic mobility, which could be because of the ionic conduction pathways aligned along the (111) orientation. The FORC-IV analysis on the LMO-LLTO VANs revealed that the LMO pillars displayed two distinctive regions, with contrasting conductivities, which could be a result of an interphase formed at the interface between LMO and LLTO. Techniques to decorrelate the high-dimensional FORC-IV data set, paving the way for quantitative analysis have been proposed. Examples on how k-means clustering, and temperature dependence measurements can be used were given, although more efforts are still required for the proper application of these methods.

In sum, the creation of a three-dimensional electrode for solid-state battery applications was exhibited, as originally proposed. Additionally, models and methods for its study and simulation were suggested. The electrochemical response, although nonconventional, was demonstrated and the observed features can be explained by the composition and dimension obtained. A follow up study should focus on different elemental ratio of the targets, which could elucidate some of the limitations. Although various epitaxial VANs have been studied, the field of lithium-based VANs for energy storage is considerably new, and these results show that it is a promising path to be followed. However, more fundamental understanding about the diffusion of Li-ions in such epitaxial VAN structures is required. Finally, the utmost goal is the synthesis of a full 3D-interdigitated battery, which would require the self-assembly of a three-component 3D nanocomposite at the center of the battery device. Such self-assembled three-component VAN is highly complex and will require an extremely subtle balance of the interaction energies between all involved materials. 


\section{Samenvatting}

Driedimensionale geometrieën voor elektroden in vastestofbatterijen vertonen een verbeterde balans tussen energie en vermogen, vergeleken met tweedimensionale elektroden. Bovendien is het interne oppervlak tussen de kathode, het elektrolyt en de anode groter, wat voor een verbeterde stroomuitvoer zorgt. Echter is de huidige fabricage van dergelijke 3D batterijen afhankelijk van kostbare of meerstaps methoden. Verticaal uitgelijnde nanocomposieten (VANs) bieden veelbelovende voordelen ten opzichte van conventionele vlakke meerlagen omdat de belangrijkste functionaliteiten beïnvloed worden door de sterke koppeling tussen de twee fases en hun raakvlakken. Epitaxiale VANs worden zelf geassembleerd door fysieke dampdepositie, zonder controle van de depositievolgorde, zoals vereist is voor vlakke meerlaagse films. In dit proefschrift werden lithium bevattende VANs van het spinel-perovskiet systeem zelf geassembleerd door middel van gepulseerde laser depositie (PLD) voor toepassing in vastestofbatterijen.

Om het elektrochemische gedrag van specifieke kristalfacetten van batterijmaterialen te verhelderen, werd de controle over de kristallografische eigenschappen van dunne films via PLD onderzocht. Verbeterde controle over de elektrochemische eigenschappen van $\mathrm{Li}_{4} \mathrm{Ti}_{5} \mathrm{O}_{12}$ (LTO), $\mathrm{LiMn}_{2} \mathrm{O}_{4}$ (LMO) en $\mathrm{Li}_{0.5} \mathrm{La}_{0.5} \mathrm{TiO}_{3}$ (LLTO) dunne films wordt mogelijk gemaakt door structurele manipulatie, dat uniek is voor epitaxiale dunne films en niet kan worden verkregen in monokristallijne of polykristallijne monsters. Grote verschillen in oppervlaktemorfologie werden bereikt door de specifieke kristaloriëntaties voor de spinel LTO en LMO dunne films te regelen. Het blootgestelde oppervlak is overwegend $\langle 111\rangle$ voor alle verkregen morfologieën en bleek de lithiumopslag van spinel dunne-filmelektroden te verbeteren vanwege significante oppervlaktebijdragen. Alle drie de kristaloriëntaties lieten hoge elektrochemische prestaties zien met een goede mogelijkheid om te laden en te ontladen zonder enige significante capaciteitsvermindering, en hoge ontladingscapaciteiten. De laagdikteafhankelijkheidsstudie toonde aan dat de grote capaciteiten veroorzaakt worden door grote oppervlaktebijdragen in lithiumopslag, waarbij de (100)-georiënteerde films de grootste oppervlakte vertoonden. Structurele manipulatie van de LLTO dunne films toonde de oriëntatieafhankelijkheid van de ionische diffusie van het vaste stof elektrolyt, aangezien de lithium-diffusieroutes in het vlak zijn georiënteerd, rekening houdend met de tetragonale eenheidscel. Activeringsenergieën voor hoppen werden berekend door een temperatuurafhankelijkheidsmeting, waarbij de bijdrage van La-rijke lagen aan de diffusie door de domeingrenzen van dunne films werd benadrukt. Tot slot werd, met de opgedane kennis van de drie componenten van de batterij, een complete vast stof batterij gegroeid. Röntgendiffractiemetingen toonden de correcte fases van LMO en LLTO. LTO vertoonde een Li-deficiënte fase, wat het gevolg kan zijn van de hogere temperaturen die benodigd zijn voor de groei van LLTO. Elektrochemische metingen laten geen 
kortsluiting zien tussen de lagen, hoewel het laden en ontladen van de volledige batterij niet mogelijk was. Optimalisatie van deze batterijen benadrukt de noodzaak van het ontwikkelen van een nauwe combinatie met een vaste elektrolyt in een verticaal uitgelijnde 3D nanocomposiet.

Omdat VANs gebaseerd op lithium nog niet onderzocht zijn voor energieopslag, is een model ontwikkeld om de vorming van deze structuren te voorspellen. De simulaties werden bovendien gebruikt om de invloed van temperatuur en depositiesnelheid op de morfologische evolutie van deze nanocomposieten te analyseren. Succesvol gebruik van een kinetische Monte Carlo simulatie (KMCS) om de groei van op lithium gebaseerde VAN films te modelleren werd gedemonstreerd, bestaande uit een veelbelovende LMOLLTO kathode-elektrolyt combinatie richting vastestofbatterijen. Een realistischer en minder rekenkracht vereisend model met een minimum aantal restricties werd toegepast, waar de interactie energieën gebaseerd zijn op experimentele reflectie hoge energie elektrondendiffractie (RHEED) metingen. De simulaties lieten goede overeenkomsten zien met de experimenteel verkregen films, en volgden dezelfde trends voor de depositiesnelheid- en temperatuur- afhankelijkheid zoals voorspeld werd door de fysieke modellen. Kwantitatieve analyse vertoonde goede overeenkomsten met betrekking tot de evolutie van de nanopilaar-dichtheid na veranderingen in syntheseparameters. Het waargenomen verschil werd verklaard door de keuze van de gebruikte energieën in het KMCS model om de rekentijd te minimaliseren. De resultaten laten zien dat KMCS modellen succesvol kunnen worden toegepast om de nanopilaar-matrixvorming te kunnen voorspellen in op lithium-gebaseerde VAN films. Echter moet hier wel voor de individuele kathode en elektrolyt materialen de specifieke kristalstructuur en stoïchiometrie in acht worden genomen, net zoals het raakvlak ertussen.

De zelfassemblageprocedure werd voor het eerst toegepast met lithium bevattende materialen om elektrode/elektrolyt nanocomposieten te maken, gedeponeerd op kristallijne substraten met PLD. De controle over de kristallografische eigenschappen van dunne VAN films, en hoedanig verschillende groeiomstandigheden de kristalstructuur, morfologie, en compositie van de nanopilaren en matrix beïnvloedden, werd aangetoond. De waargenomen trend in de temperatuurafhankelijkheidsstudie suggereerde de formatie van de Ti-gedoteerde $\mathrm{LiMn}_{2-\mathrm{y}} \mathrm{Ti}_{\mathrm{y}} \mathrm{O}_{4}$ fase, met $\mathrm{y}=1.5$, wat later bevestigd werd met reciprocal space maps (RSM) en elektronen energieverlies spectroscopie (EELS) analyse. De aanwezigheid van $\mathrm{Ti}$ in de LMO pilaren kan het resultaat zijn van de lagere vormingsenthalpie van LMTO. De depositiesnelheid, die gebruikt kan worden om de pilaargrootte van de VANs aan te passen, bleek nauwelijks effect te hebben op de samenstelling van de LMO fase. Verschillende substraatoriëntaties kunnen gebruikt worden om de structuur van de matrix en de pilaar fases en als gevolg, de morfologie en het raakvlak tussen de twee, aan te passen. Gebaseerd op experimentele resultaten en de simulaties kan de activeringsenergie voor het hoppen van de geadsorbeerde atomen op het substraatoppervlak worden aangepast door middel van verschillende bufferlagen, wat resulteert in een A- of B-terminatie. Deze verandering in diffusiviteit werd gebruikt om 
de pilaargrootte af te stemmen en het pilaar-stroomverzamelcontact te vergroten. De groei op een Si-substraat, gebufferd met een kristallijne laag STO, werd aangetoond, dat wijst op de mogelijkheid van integratie met Si-technologie. De verwachtte karakteristieke pieken waren niet zichtbaar in de elektrochemische analyse, wat suggereert dat de in dit hoofdstuk bestudeerde VANs een complex systeem zouden kunnen opleveren dat de mogelijkheden van de huidige elektrochemische technieken te boven gaat.

Geavanceerde scanning probe microscopie (SPM) technieken maken het mogelijk elektrochemische metingen op de nanoschaal uit te voeren, welke gebruikt kunnen worden om de structuur/functie verhoudingen in batterijmaterialen met uitzonderlijke resolutie op te helderen. Om inzicht in de niet-uniforme verdeling van de lithiumactiviteit te krijgen, werd een first order reversal curve current-voltage (FORC-IV) analyse toegepast. De geleidingsmechanismen die de elektrochemie op de nanoschaal overheersen werden bestudeerd door middel van lokale ionische diffusie in LMO en dunne VAN films die epitaxiaal gegroeid werden met PLD. De FORC-IV piekspanning mappen toonden aan dat het $\langle 111\rangle$ facet de hoogste ionische mobiliteit vertoont, dat zou kunnen komen door de ionische geleidingsroutes die zijn uitgelijnd langs de (111)oriëntatie. De FORC-IV analyse van de LMO-LLTO VANs onthulde dat de LMO pilaren twee onderscheidende regio's vertoonden met contrasterende geleidingen, wat het resultaat zou kunnen zijn van een gevormd raakvlak tussen de LMO en LLTO. Er zijn technieken voorgesteld om de hoog-dimensionale FORC-IV dataset te decorreleren en zo de weg vrij te maken voor kwantitatieve analyse. Voorbeelden werden gegeven van hoe k-means clustering en temperatuur-afhankelijkheidsmetingen gebruikt kunnen worden, hoewel er nog meer inspanning nodig is voor de juiste toepassing van deze methoden.

Kortom, de creatie van een driedimensionale elektrode voor vastestofbatterijtoepassingen werd getoond, zoals oorspronkelijk voorgesteld. Bovendien werden modellen en methoden voor de studie en simulatie ervan voorgelegd. De elektrochemische respons, hoewel onconventioneel, werd aangetoond en de waargenomen kenmerken kunnen verklaard worden door de verkregen compositie en afmetingen. Een vervolgstudie zou zich moeten concentreren op verschillende elementaire verhoudingen van de targets, wat enkele van de beperkingen zou kunnen ophelderen. Hoewel verschillende epitaxiale VANs bestudeerd zijn, is het vakgebied van op lithium gebaseerde VANs voor energieopslag aanzienlijk nieuw, en deze resultaten laten zien dat het een veelbelovend pad is om te volgen. Echter is er meer fundamentele kennis benodigd over de diffusie van Li-ionen in zulke epitaxiale VAN structuren. Ten slotte is het grootste doel de synthese van een volledige 3D verwoven batterij, waarvoor de zelfassemblage van een drie-componenten 3D nanocomposiet in het midden van een batterij benodigd is. Dergelijke zelf-geassembleerde drie-componenten VAN zijn uiterst complex en vereisen een extreem subtiele balans tussen de interactie-energieën van alle betrokken materialen. 


\section{List of Publications}

- Singh, D., Cunha, D. M., Dubbelink, T., Hendriks, T. A., Lievens, C., Huijben, M., Enhanced cycling and rate capability by epitaxially matched conductive cubic TiO coating on $\mathrm{LiCoO}_{2}$ cathode films, ACS Applied Energy Materials, in revision.

- Cunha, D. M., Huijben, M., Lithium-based Vertically Aligned Nanocomposites for 3D Solid-state Batteries, MRS Bulletin, (2021). https://doi.org/10.1557/s43577-02100026-2

- Xia, R., Sun, C., Wang, Y., Cunha, D. M., Peng, H., Zhao, K., Huijben, M., ten Elshof, J. E., Enhanced lithiation dynamics in nanostructured $\mathrm{Nb}_{18} \mathrm{~W}_{16} \mathrm{O}_{93}$ anodes, Journal of Power Sources, 482, 228898 (2021).

- Chen, B., Gauquelin, N., Jannis, D., Cunha, D. M., Halisdemir, U., Piamonteze, C., Lee, J. H., Belhadi, J., Eltes, F., Abel, S., Jovanovic, Z., Spreitzer, M., Fompeyrine, J., Verbeeck, J., Bibes, M., Huijben, M., Rijnders, G., Koster, G., Strain-Engineered Metalto-Insulator Transition and Orbital Polarization in Nickelate, Advanced Materials, 32, 2004995 (2020).

- Cunha, D. M., Vos, C. M., Hendriks, R., Singh, D. P., Huijben, M., Morphology Evolution during Lithium-Based Vertically Aligned Nanocomposite Growth. ACS Materials and Interfaces, 11 (47), 44444-44450 (2019).

- Cunha, D. M., Hendriks, R., Vasileiadis, A., Vos, C. M., Verhallen, T., Singh, D. P., Wagemaker, M., Huijben, M., Doubling reversible capacities in epitaxial $\mathrm{Li}_{4} \mathrm{Ti}_{5} \mathrm{O}_{12}$ thin film anodes for microbatteries. ACS Applied Energy Materials, 2 (5), 3410-3418 (2019).

- Hendriks, R., Cunha, D. M., Singh, D. P., Huijben, M., Enhanced lithium transport by control of crystal orientation in spinel $\mathrm{LiMn}_{2} \mathrm{O}_{4}$ thin film cathodes. ACS Applied Energy Materials, 1 (12), 7046-7051 (2018).

- Cunha, D. M., Souza, F. L., Facile synthetic route for producing one-dimensional zinc oxide nanoflowers and characterization of their optical properties. Journal of Alloys and Compounds, 577, 158-164 (2013).

- Cunha, D. M., Ito, N. M., Xavier, A. M., Arantes, J. T., Souza, F. L., Zinc oxide flower-like synthesized under hydrothermal conditions. Thin Solid Films, 537, 97-101 (2013). 


\section{Acknowledgements}

Any intellectual progress relies on the understanding gained by previous thinkers. While this is a fact, supported by the +200 references cited which without I would not be able to write a thesis, I believe it also holds true for all other non-academic influences. This thesis is a result of experiences shared with many people, that created the circumstances for me to be exactly where I am, and that I would like to acknowledge here. Although I would like to thank every single person, for the purpose of this thesis I will mention a few.

I would like to express my gratitude to the Inorganic Materials Science group for the opportunity of carrying out my work, for its facilities, coffee breaks, football matches and friendly environment. I wish I were able to thank everyone... and so I will try. So, thanks to Guus, Bernard, Evert, Gertjan, Monica, Rebecca, Laura B.-L., Laura V., Ben, Binbin, Minh, Pierre, Yorick, Sina, Kit, Fox, Phú, Shu, Shweta, Yury, Tatiana, Rui, Jie, Jun, Petra, Alim, Kurt, Werner, Gerard, Anirban, Huiyu, and many master and bachelor students. Thanks to Ron, which I shared my work, the time in the lab, and most of the conferences. A special mention to Deepak, for sharing his knowledge and experience, and showing me ways of the battery. A shout-out to my awesome office mates, Thanh and Yang. A big thanks to Marion, always helpful and caring, and to our technical staff, Harry, Karin, and Jose, which certainly without their help I would need four more years to get anything done. An extended acknowledgement to Frank, Nicolas G. and Mark S. for all their help on the lab or for the beautiful pictures.

I am especially grateful for my students, Katrina, Chris, Arnoud, and Thijs, that made worth all the hours spending on correcting reports. Their work is present on these pages and without them this thesis would be two chapters short.

My gratitude to my daily supervisor, which despite of all my e-mail harassment, allowed me to come back and through his efforts made it possible for me to stay. Mark, you have shaped my life and, consequently, many others in an extent that you probably do not even imagine. Thank you.

I would like to acknowledge Ufuk, Marie and Lucie, Pablo and Joanna, David, and Dominic for all the great insights and knowledge that certainly helped me in my time here, but also for all the meaningful conversations and the silly ones too. You brightened my days.

Thanks to Marijn, Melvin, Moritz and Ditsy for our musical experience in the Rocking Curve and for our beer, burgers, and band practice nights. You guy makes Thursday (and later, Tuesdays) the best day of the week. For Marijn and Melvin, my two favorite allies (and paranymphs), that share the tanktop fashion and the groove, I say: Goonay-nay. 
To Wesley, Paula, Joshua, Jayden, Ans and Klaas, thank you for being my home away from home, and giving me the family feeling that makes being alone in a foreign country bearable.

Eu gostaria de agradecer aos meus dois grandes amigos Vitor e Matheus. Vitor, você é a razão de eu estar aqui. Obrigado pela sabedoria, orientação, pelas conversas, e pelos momentos compartilhados. Matheus, obrigado por estar presente. Inquestionavelmente você é responsável por manter minha saúde mental em níveis aceitáveis nos últimos tempos. Foco no Algarve. Tchamo.

E finalmente, eu agradeço aos meus pais, Rachel e Marcos, e à minha irmã, Natalia. Toda e qualquer conquista minha é um resultado direto do seu apoio e sacrifício durante minha jornada. Eu não estaria onde estou sem o seu suporte. Essa tese é dedicada a vocês. Muito obrigado.

Daniel M. Cunha

Enschede, July, 2020 